\title{
NumericAl Simulation OF ACOUSTiC STREAMing WiTHiN THE COCHLEA
}

Von der Fakultät für Elektrotechnik und Informatik der Gottfried Wilhelm Leibniz Universität Hannover zur Erlangung des Grades Doktor der Naturwissenschaften Dr. rer. nat. genehmigte Dissertation von

Dipl.-Math. Christian Gerstenberger geboren am 30.01.1983 in Hannover 
Referent: Prof. Dr. F.-E. Wolter, LUH

Koreferentin: Prof. Dr. N. Magnenat-Thalmann, Univ. Genf und NTU Koreferent: Prof. H. Matthies, TU Braunschweig

Tag der Promotion: 18. September 2012 


\section{Acknowledgement}

First of all I want to thank my doctoral advisor Prof. Franz-Erich Wolter for his support and the intense discussions that helped to reflect the own research activities. I am also very grateful that it was possible to get a scholarship from the Welfenlab of the Leibniz University Hanover. Moreover, I want to thank Prof. Nadia Magnenat-Thalmann and Prof. Hermann G. Matthies for being my co-advisors. I would also like to thank all the members of the Welfenlab research group for the helpful discussions and the encouragement.

Particular thanks are due to my wife, Rebecca, as well as my parents for their continuous love and support throughout the entire process. Most importantly, I thank God for giving me the possibility and the talents to accomplish these doctoral studies. 


\begin{abstract}
This work is concerned with the numerical examination of acoustically driven flows within the inner ear on the basis of a computational model. For this purpose, a comprehensive system of differential equations and boundary conditions is deduced, which takes, to a satisfactory extent, the complexity of the main biophysical mechanisms of the cochlea into account.

Beside an appropriate representation of the fluid dynamics, also the biomechanical properties of the basilar membrane as well as the internal amplification mechanism caused by the outer hair cell motility are considered in order to get realistic estimates of the structure and magnitude of the mean flow field. In the present work, the interior fluids are modeled on the basis of a two-dimensional simplified domain by using concepts from the field of continuum mechanics. According to an approach from Mammano and Nobili [28, the mechanical reaction of the cochlear partition and also the outer hair cell motility are represented as one-dimensional oscillatory systems. With the aid of the perturbation theory, two linear subproblems are extracted that can be used for a separate determination of the acoustic and the acoustic streaming field.

The present work introduces a two-stage approach for the numerical evaluation of the solutions on the basis of the finite element method. The first step deals with the time-dependent acoustic subproblem. Due to the strong coupling between the different components a monolithic approach is considered that simultaneously calculates the interacting processes. The second step is associated with the stationary acoustic streaming subproblem that provides a first order approximation of the acoustic streaming field.

Finally, the numerical solutions of the computational model are presented. It is shown that the results are essentially consistent with measurements as well as analytical and experimental considerations.
\end{abstract}

Keywords: acoustic streaming; cochlea; fluid-structure-interaction. 


\section{Zusammenfassung}

Die vorliegende Arbeit beschäftigt sich mit der numerischen Untersuchung von akustisch induzierten Strömungen innerhalb des Innenohres auf Grundlage eines Berechnungsmodells. $\mathrm{Zu}$ diesem Zweck wird ein umfassendes System aus Differentialgleichungen und Randbedingungen abgeleitet, das der Komplexität der biophysikalischen cochlearen Vorgänge gerecht wird.

Um möglichst realistische Ergebnisse in Bezug auf die Struktur und Größenordnung der akustisch induzierten Strömungen zu erhalten, werden neben einer angemessenen Darstellung der Fluiddynamik zudem auch die biomechanischen Eigenschaften der Basilar Membran sowie der interne Verstärkungsmechanismus - der durch die Bewegungen der äußeren Haarzellen hervorgerufen wird - berücksichtigt. Die inneren Flüssigkeiten werden in dieser Arbeit mithilfe von Konzepten der Kontinuumsmechanik auf einem simplifizierten zweidimensionalen Gebiet modelliert. Die mechanische Rückkopplung der Basilar Membran als auch die Bewegung der äußeren Haarzellen werden, entsprechend eines Ansatzes von Mammano und Nobili [28], jeweils durch ein eindimensionales schwingungsfähiges System beschrieben. Mithilfe der Störungstheorie werden schließlich zwei lineare Teilprobleme gewonnen, mit denen jeweils eine separate Bestimmung der akustischen Schwingungen und der akustisch induzierten Strömung möglich wird.

Die vorliegende Arbeit stellt ein zweistufiges Verfahren zur numerischen Bestimmung der Lösungen auf Grundlage der Finite-Elemente-Methode vor. Die erste Stufe behandelt das akustische Teilproblem. Aufgrund der starken Kopplung zwischen den unterschiedlichen Komponenten wird ein monolithischer Ansatz verwendet, der die interagierenden Prozesse simultan berechnet. Die zweite Stufe liefert im Anschluss für das zweite Teilproblem eine Näherungslösung erster Ordnung für die akustisch induzierten Strömungen.

Zum Abschluss werden die numerischen Ergebnisse des Berechnungsmodells dargelegt. Darüber hinaus wird gezeigt. dass die Resultate im Wesentlichen mit Messungen sowie analytischen und experimentellen Untersuchungen übereinstimmen.

Schlüsselwörter: akustisch-induzierte Strömungen; Cochlea; Fluid-Struktur-Interaktion. 


\section{Contents}

1 Introduction 1

2 Modeling 7

2.1 Simplifying Assumptions . . . . . . . . . . . . . . . . . . . . . 9

2.2 The Fluid Dynamics . . . . . . . . . . . . . . . . . . . . . . . 11

2.2 .1 Conservation Equations . . . . . . . . . . . . . . . . . . 12

2.2 .2 Constitutive Relations . . . . . . . . . . . . . . . . . . . 13

2.2.3 Boundary Conditions . . . . . . . . . . . . . . . . . 15

2.2 .4 Perturbation Expansion . . . . . . . . . . . . . 16

2.3 The First Order System . . . . . . . . . . . . . . . . . . . 17

2.3.1 The Acoustic Subproblem . . . . . . . . . . . . . . 18

2.3.2 The Passive Mechanics of the Cochlear Partition . . . . . . . . 18

2.3.3 Outer Hair Cell Motility . . . . . . . . . . . . . . . . . . . . . . . 24

2.3 .4 Equilibrium state . . . . . . . . . . . . . . . . . 2 29

2.4 The Second Order System . . . . . . . . . . . . . . . . . . 31

2.4.1 The Acoustic Streaming Subproblem . . . . . . . . . . . . . . . 31

3 Implementation 33

3.1 Mesh Generation . . . . . . . . . . . . . . . . . 35

3.1 .1 Reference Elements . . . . . . . . . . . . . . . . . . . 36

3.1 .2 Refinement of the mesh . . . . . . . . . . . . . . . . 37

3.1 .3 Shape of the Mesh . . . . . . . . . . . . . . . . . . 40

3.1.4 Basilar Membrane as a Submesh . . . . . . . . . . . . . . . 41

3.2 Variational Formulation . . . . . . . . . . . . . . . . . 41

3.2 .1 The First Order System . . . . . . . . . . . . . . . . 43

3.2 .2 The Second Order System . . . . . . . . . . . . . . . . 45

3.3 Function Spaces . . . . . . . . . . . . . . . . . . . . . . . . . 46

3.3.1 Spatial Solution Spaces . . . . . . . . . . . . . . 46

3.3.2 Local Finite Element Spaces . . . . . . . . . . . . . . . . . . . . . . . . . . . . . . . . . . . . . .

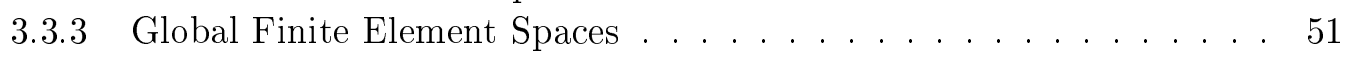

3.4 Spatial Discretization . . . . . . . . . . . . . . . . . 53

3.4 .1 The First Order System . . . . . . . . . . . . . . 5 53

3.4.2 Multiphysical Coupling . . . . . . . . . . . . . . . 60 60 
3.4.3 The Second Order System . . . . . . . . . . . . . . . . . . 63

3.4.4 Assemblage . . . . . . . . . . . . . . . . . . . . . 66

3.5 Temporal Discretization . . . . . . . . . . . . . . . . 68

3.6 Linear Solver . . . . . . . . . . . . . . . . . . . . . . 70

3.6.1 Generalized Minimal Residual Method . . . . . . . . . . . . . . 71

3.6 .2 Preconditioning . . . . . . . . . . . . . . . . . 75

3.6 .3 Incomplete LU decomposition . . . . . . . . . . . . . . . . 75

3.7 Sine-Cosine Decomposition ． . . . . . . . . . . . . . . 77

4 Results 79

4.1 Harmonic Stimulation of the First Order System . . . . . . . . . . . . . . 80

4.1 .1 Initial Transient Effect . . . . . . . . . . . . . . . . . . . 80

4.1.2 Forces acting on the Basilar Membrane . . . . . . . . . . . . . . . 81

4.1.3 Traveling Wave on the Basilar Membrane . . . . . . . . . . . . . 86

4.1.4 Motions within the Stokes Boundary Layer . . . . . . . . . . . . . . 90

4.2 Fluid Flows driven by the Second Order System . . . . . . . . . . . . . . . 93

4.2.1 Difference between Eulerian and Lagrangian Mean Motions . . . . . 93

4.2 .2 Mass Source driven Streaming . . . . . . . . . . . . . . . . . . . 96

4.2 .3 Force Source Driven Streaming . . . . . . . . . . . . . . . . 9979

4.2 .4 Békésy's eddies . . . . . . . . . . . . . . . . . . . . . . . . . 999

5 Conclusion $\quad 103$

A Results of the Passive Cochlea Model $\mathbf{1 0 7}$

A.1 Initial Transient Effect . . . . . . . . . . . . . . . . . . . . . . . . . 107

A.2 Rotary Vibrations at Equilibrium State . . . . . . . . . . . . . . . . 112

A.3 Displacement of the Basilar Membrane . . . . . . . . . . . . . . . . 119

A.4 Acoustic Streaming Flow Field . . . . . . . . . . . . . . . . . . 123

B Results of the Active Cochlea Model $\quad \mathbf{1 2 7}$

B.1 Acoustic Flow Fields at Equilibrium State . . . . . . . . . . . . . . . . . . 128

B.2 Displacement of the Basilar Membrane . . . . . . . . . . . . . . . 135

B.3 Acoustic Streaming Flow Field . . . . . . . . . . . . . . . . . . . . . . . 139

\begin{tabular}{ll} 
List of Figures & 143 \\
\hline
\end{tabular}

List of Tables 146

List of Algorithms

Bibliography $\quad \mathbf{1 5 0}$

Index 155 


\section{Chapter 1}

\section{Introduction}

The process of hearing is a quite complex mechanism which is still not fully understood. The auditory system of humans is capable of recognizing acoustic signals between $20 \mathrm{~Hz}$ and $20 \mathrm{kHz}$ and a sensitivity range of about 12 orders of magnitude (cf. [38]). As illustrated in figure 1.1, the soundwave has to pass initially the outer ear, the external auditory canal as well as the middle ear (which consists of the tympanic membrane, the malleus, the incus and the stapes) before reaching the snail-shaped inner ear, which is known as cochlea (cf. figure 1.2). Within the cochlea the acoustic signal is converted into nerve impulses by an elaborate bio-mechanical mechanism. This mechanism as well as the triggering process of the nerve impulses are mainly caused by the deflection of tiny hair-bundles, the so-called stereocilia. In the light of this underlying processes, the relevance of acoustic streaming and its potential influence on the bio-mechanical mechanisms are still an open issue.

The term acoustic streaming is associated with the mean motions of a fluid or a gas that are induced by an acoustic field. Up to now, a direct examination of such mean flows within the cochlea has not yet been possible due to the limited accessibility and the small dimensions of the liquid filled chambers. Furthermore, it can be expected that the resultant velocities of the acoustic streaming field are relatively small in comparison to the characteristic velocities of the sound field. This work yields numerical estimates of the magnitudes as well as the structure of acoustic streaming within the cochlea on the basis of a simulation process. The objective of this computational model is the provision of a new instrument for a substantiated discussion on the physiological impact of acoustically driven flows within the cochlea. In addition, a completely new approach for simulating acoustic streaming within a fluid-structure coupled system like the inner ear is proposed which might also be used in a wide range of other applications.

The physical origin of acoustic streaming can be found in different dissipation mechanisms of acoustic energy. The propagation of sound waves is usually accompanied by a mean momentum flux (better known as Reynolds stress) and a mean mass flux. Such a mean net rate is caused when the transport of momentum, or the transport of mass respectively, across an unit area is unbalanced over one cycle (in the case of a pure-tone 


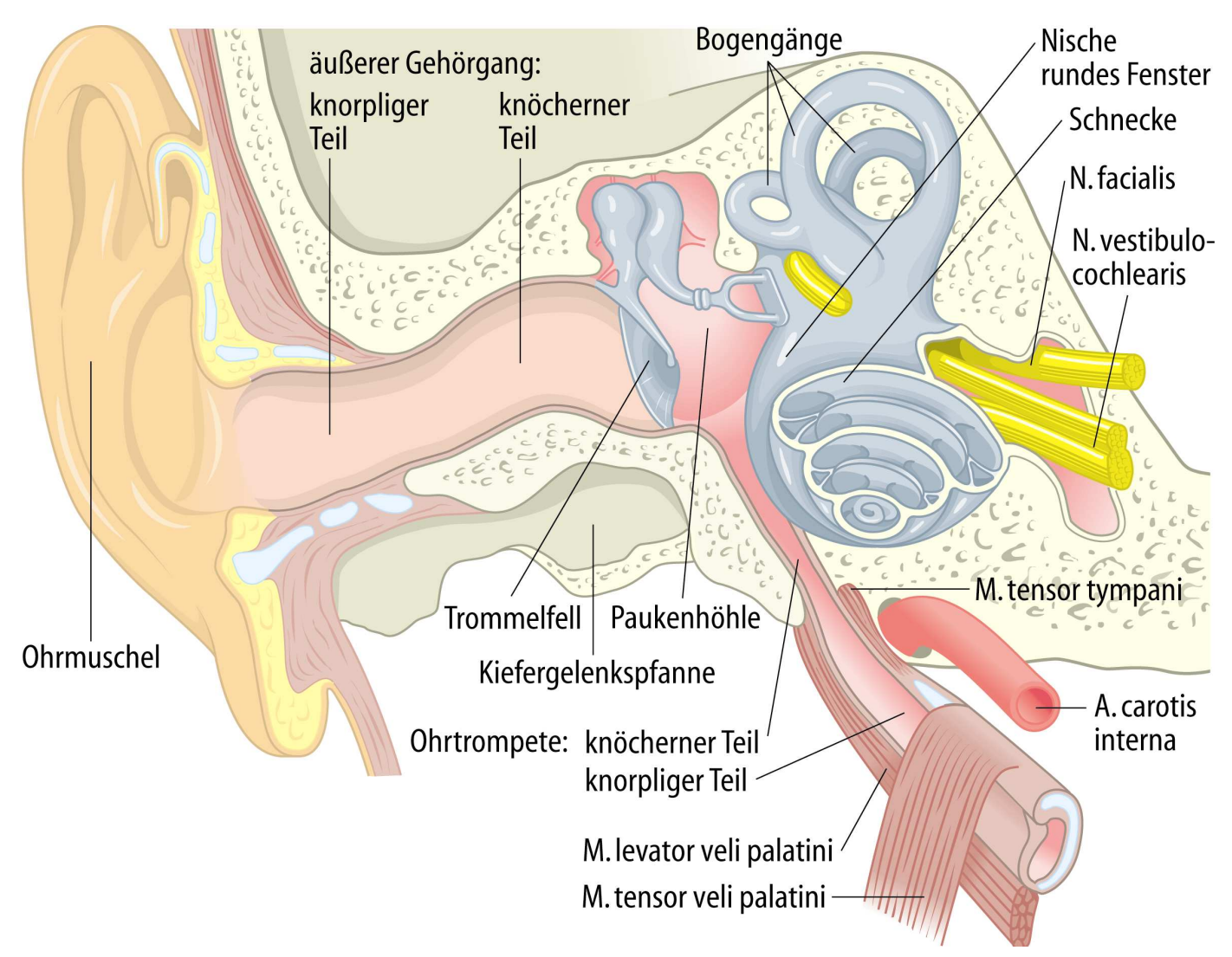

Figure 1.1: Structure of the human ear. (source Boenninghaus and Lenarz [4])

stimulation). As a result of the sound wave attenuation it can be expected that also the mean momentum flux as well as the mean mass flux are weakened at regions of acoustic energy dissipation. The excess mass that can no longer be transported by the sound beam must be released and it appears as if this mass is injected within the fluid. In the same way, the excess momentum appears as an additional force acting on the fluid. In principle, these virtual mass sources or virtual force sources are the main cause of acoustically driven flows. (cf. [26, 9, 6])

Viscosity can, for example, be identified as one major kind of the dissipation mechanisms. But, viscous attenuation of acoustic energy typically becomes significant either at high frequencies or next to boundaries due to an enhanced friction of adjacent fluid layers. Since typical frequencies of the cochlear system are comparatively low, only viscous attenuation within the boundary layers can be considered as a cause for substantial mean flows. Even though the dissipation of acoustic energy is restricted to such a boundary layer, a remarkable mean flow can also occur outside of this boundary layer. A fundamental analysis of these boundary driven flows goes back to Lord Rayleigh [35, §352]. He calculated an effective slip flow in order to represent the mean velocity of the fluid at the edge of the boundary layer relative to the boundary. In this context, it is interesting to note that although the effective slip flow is caused by viscosity, the dimension of this motion does 


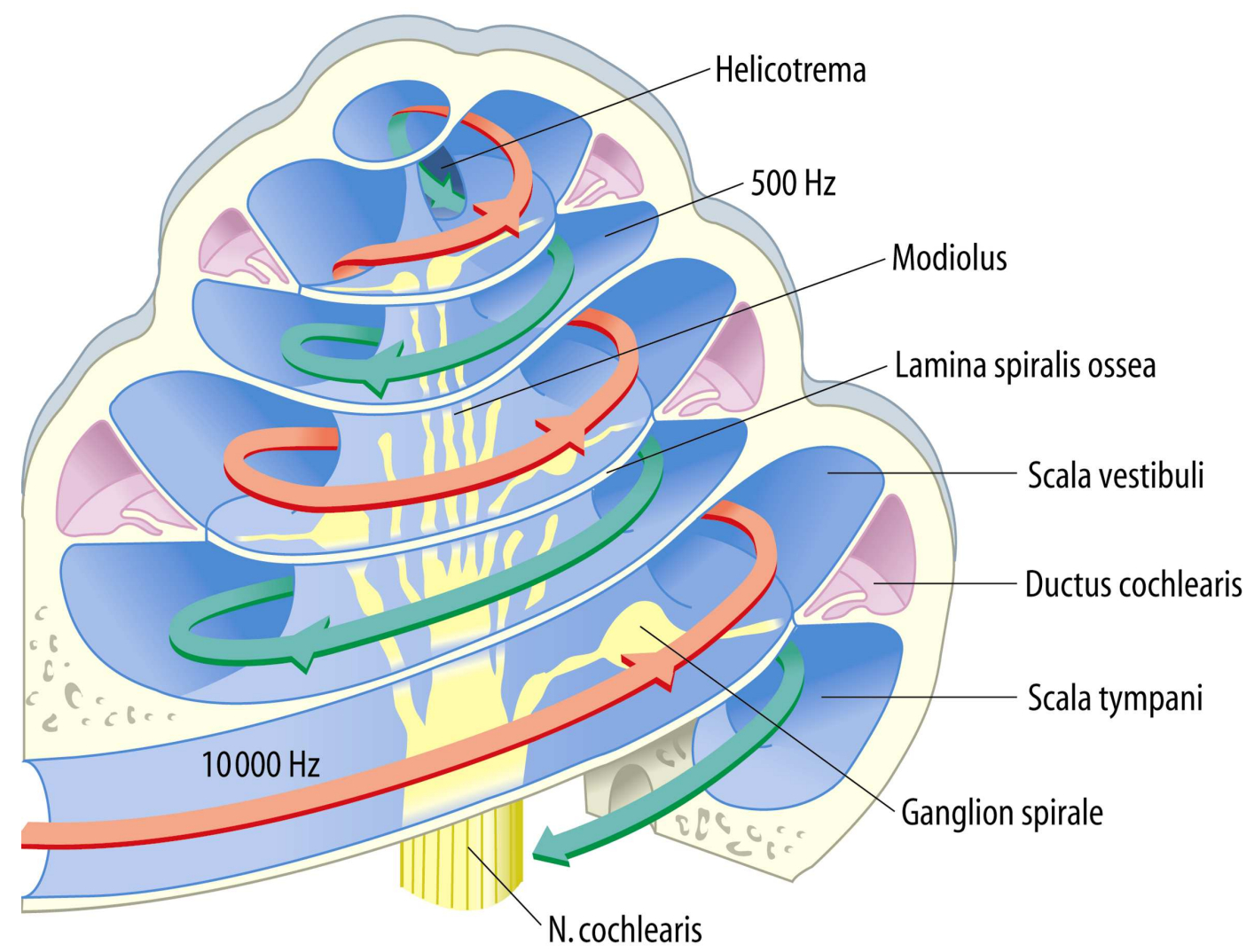

Figure 1.2: Structure of the cochlea. (source Boenninghaus and Lenarz [4])

not depend on an associated viscosity-coefficient. The reason for this phenomenon lies in the fact that the resistance to such a mean flow also depends on the viscosity itself. (cf. [26, 35]) Therefore, even if the viscosity of a fluid is small, the resulting effective slip flow velocities can become substantial.

Two very important contributions to the subject of acoustic streaming within the inner ear, which arise from the cochlear travelling waves, come from Hallauer [20] and Lighthill 25. Hallauer as well as Lighthill examined the acoustic driven flows on the basis of theoretical considerations. Both approaches use mathematical asymptotic and perturbation techniques in order to get approximations of the nonlinear fluid motion within the cochlea. While Lighthill focusses on the determination of an estimate of the potential velocities of acoustic streaming, Hallauer also calculates the streamlines of the acoustically driven motions on the basis of his mathematical model.

In contrast to the theoretical considerations from Hallauer and Lighthill, an experimental examination of acoustic streaming can be traced back to Békésy [50]. In his pioneering work he performed inter alia studies on mechanical cochlea models. He observed that above and below the cochlear partition two eddies arise if the mechanical model is stimulated by a sinusoidal excitation. It turns out that this eddy-like motion can be associated with the 


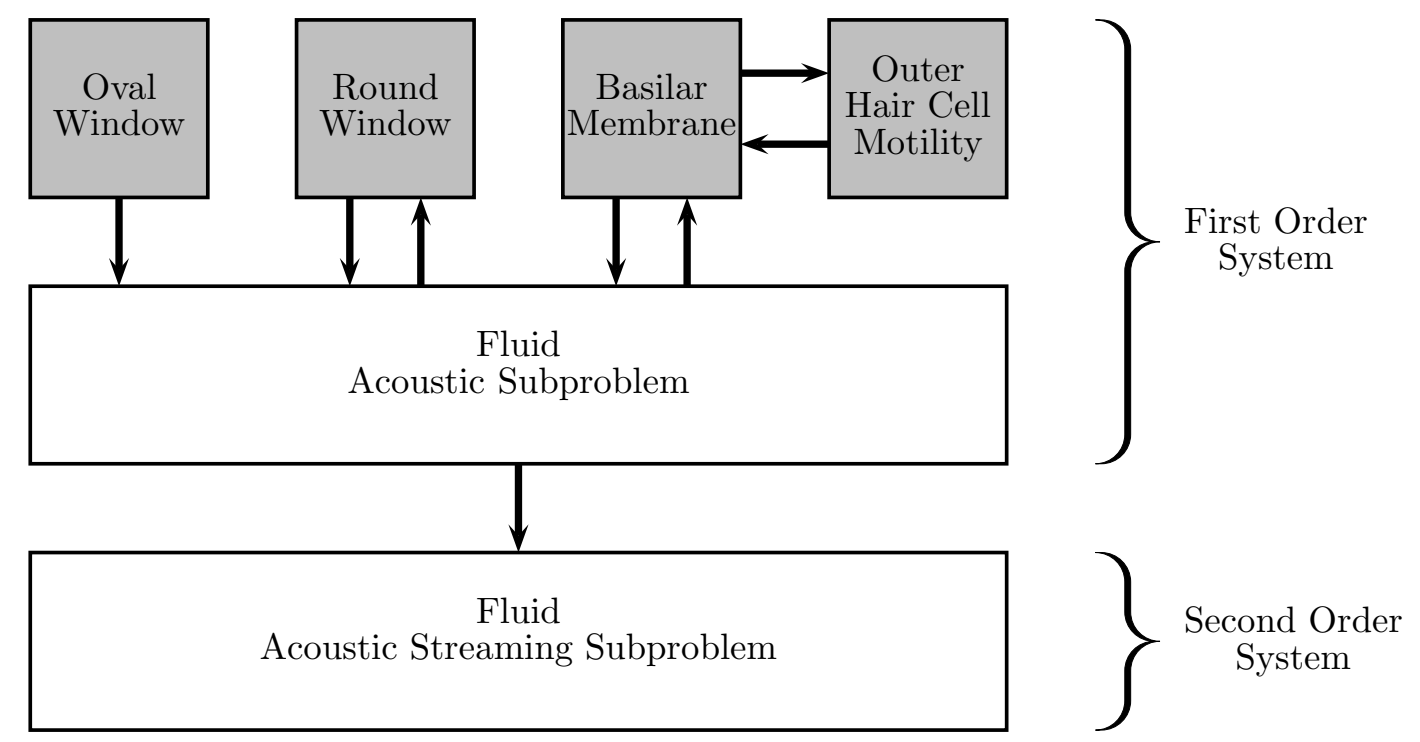

Figure 1.3: Schematic layout of the computational model used for simulation of acoustically driven flows within the cochlea. On the basis of a perturbation technique, the system is separated into first and a second order system. The first order system describes the linear acoustic field of the fluid as well as its interactions with the structures, such as the oval window, the round window and the basilar membrane. Furthermore, the motility of the outer hair cells is taken into account which results in additional forces acting on the basilar membrane. The first order system provides results that can be used by the second order system in order to calculate an approximation of acoustic streaming.

term acoustic streaming. Each eddy is characterized by an apical directed mean flow of the fluid-particles that are located close to the cochlear partition and a basal directed mean motion at the outer boundaries. The longitudinal position of the eddies depends on the frequency and correlates with the characteristic place. (cf. [50, 48]) A detailed description of these eddy-like mean flows was carried out by Tonndorf [48].

Acoustic streaming has already been studied in a lot of numerical simulation processes. Different kinds of numerical approaches for the simulation of acoustic streaming were, for example, reviewed by Boluriaan and Morris [6]. But up to now, it has not yet succeeded to establish an adequate method for the numerical simulation of acoustic streaming within biophysical systems like the cochlea. The primary reason for this lies in the fact that the velocity field of the fluid is mainly influenced by the interactions with its adjacent structures.

In this work a well-proven numerical method is adapted that was successfully implemented by Köster [24, who studied the structure of acoustic streaming caused by piezoelectrically driven microfluidic biochips. Köster used a standard perturbation technique in order to split the nonlinear acoustic streaming problem into two linear subproblems. His computational model also includes a more precise treatment of the acoustic radiator, as originally suggested by Bradley [9].

To investigate the influence of acoustically driven flows in the inner ear, an appropriate 
computational model was developed which has been studied in a simulation process. In contrast to the work from Köster [24, this computational model consists not only of a fluiddomain but also of structural components that have a significant influence on the fluid. A schematic layout of the model is shown in figure 1.3. As one can see, the model introduced in this thesis can also be divided into a first order system and a second order system. The first order system describes the linear acoustics within the fluid-domain in combination with the mutual interactions of the cochlear structures, such as the basilar membrane, the oval window and the round window. The oval window serves as an acoustic radiator due to the direct contact with the stapes of the middle ear. The round window is covered by a membrane that allows the fluid to move arbitrarily to a certain degree. The basilar membrane can also be brought into motion due to the difference of the pressure above and below the cochlear partition. In turn, such a displacement of the basilar membrane also has an influence on the velocity field of its adjacent fluid. Furthermore, an additional force term that acts on the basilar membrane has also to be taken into account for the first order subproblem in order to represent the motility of the outer hair cells. At a second stage the acoustically driven flows can be determined on the basis of the results of this first order system.

As illustrated in figure 1.4, the analysis of a dynamic system by means of the development and application of an appropriate simulation model consists of different steps. First of all, the main purpose and the principal targets of the model should be clearly specified by a problem formulation. In this work, the following statement serves as such a guideline that summarizes the preliminary considerations and remarks:

The model is intended to verify, whether a significant mean flow occurs. The simulation process should estimate the order of magnitude of these mean motions as well as its structure.

On the basis of a problem formulation, an abstract model is usually designed by making reasonable simplifications, collecting specific data and establishing evaluable mathematical relations. In this context, it is important to ensure that the basic assumptions and simplifications at this stage of modeling lead to a valid model with regard to its purpose. The basic mathematical model, used in this work, is presented in chapter 2. In this context, fundamental aspects with respect to the anatomical dimensions as well as the macro-mechanical characteristics of the fluid and the cochlear partition are taken into account. If no analytical solutions of the abstract mathematical model are found, the model can be transformed into a computer-recognizable form (also referred to as computational model) in order to obtain numerical approximations. The conversion of the abstract cochlea model into a computational model is described in chapter 3. The implementation is realized by the use of the finite element method that mainly comprises the establishment of a so-called variational formulation, the consideration of the solution spaces, a spatial and temporal discretization and the assemblage of the resultant discretized systems of equations that can be solved numerically. In order to verify that the model accurately represents the essential features of the real system, the model has to undergo some predefined experiments for the purpose of validation. If the results of these validation-experiments are not satisfying, 


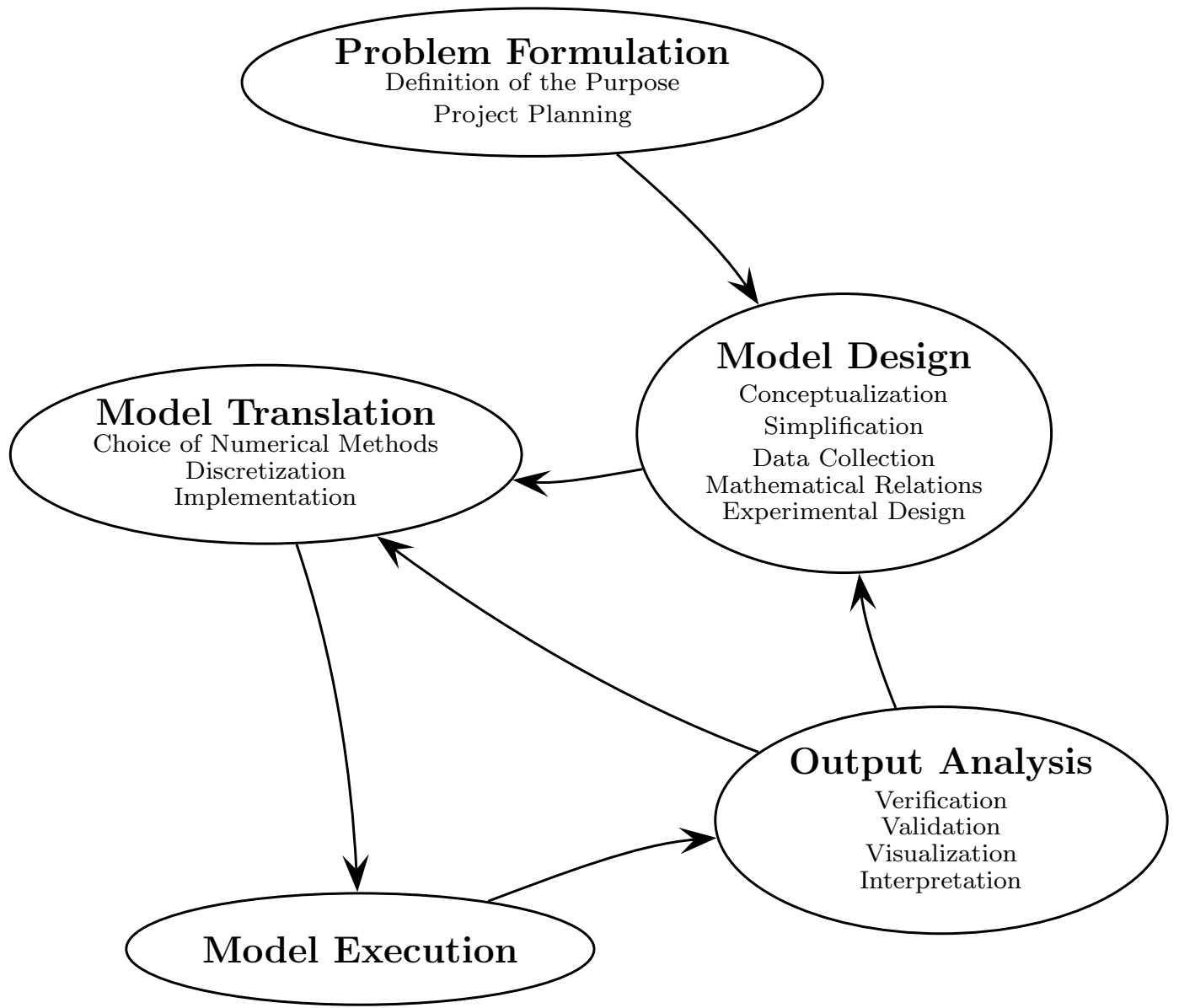

Figure 1.4: This figure illustrates the principal and underlying process of the development of the cochlea model presented in this work. As it can be seen, the modeling process is characterized by an iterative process involving the model design, the model translation, the model execution and the output analysis.

either the basic mathematical model or the implementation should be adapted. Therefore, the development and application of a simulation model is an iterative process involving the basic modeling, the implementation, the execution and the validation. Chapter 4 describes and analyzes different experiments which were performed to validate the correct functionality of the computational model. In this context, the cochlear model was compared with the mammalian cochlea, mechanical cochlear models and theoretical considerations. The final results concerning acoustic streaming can also be found in this chapter.

This thesis is based on an article that is submitted for publication in an upcoming issue of the Journal of Computational Acoustics. In contrast to this article, the present work is supplemented by a lot of additional background informations concerning the modeling-, implementation- and validation-process. 


\section{Chapter 2}

\section{Modeling}

Most people associate the word "model" with a physical, often smaller and simplified clone of an original object which can be studied for example in wind tunnels or swimming pools. These types of models are known as iconic models. But in general, a model is a representation of an object or a system. Therefore, also illustrations, functional diagrams, sectional views, mathematical relationships, or electronic circuits can be regarded as models.

Especially in the last few decades the importance of computational models has increased with the possibility of the execution of computationally intensive calculations by computers. Computational models are based on evaluable mathematical relations that can normally not be solved analytically. In such a case, it is necessary to determine the solution by the use of numerical methods. In contrast to other kinds of models, they are easy to manipulate and the influence of parameters can be simple examined. But the determination of the mathematical relations and physical laws as well as the numerical realization requires a lot of system knowledge, expertise and experience.

In general, models can be characterized by different (contrary) properties (cf. e.g. 8]). The most important aspects are given by the following items:

- explanatory $\leftrightarrow$ descriptive: An explanatory model tries to emulate the internal mode of action of the system. A descriptive model by contrast tries to imitate the behavior of the system without the knowledge of the internal mode of action. According to a black box, the imitation is only based on the observation of the system.

- static $\leftrightarrow$ dynamic: Dynamic models take time-dependent processes of the system into account, whereas a static model is characterized by its time-invariance.

- system parameter $\leftrightarrow$ parameter-adaption: If the internal (physical) quantities and dimensions of a system are known or can be measured, they can be directly used as characteristic model parameters. Otherwise, if some quantities cannot be determined, the unknown parameters might be adjusted by an iterative process as long as the behavior of the model does not correspond to the behavior of the system. 
- deterministic $\leftrightarrow$ stochastic: In stochastic models, the influence of probabilistic and random effects are taken into account. Otherwise, a model will be called deterministic if the behavior of the model is clearly predetermined by the external influences.

- time-continuous $\leftrightarrow$ time-discrete: The state of time-discrete models is only defined at separated points in time. In contrast, the state variables of a time-continuous model can be determined at any time within a specified period of time.

- continuous space $\leftrightarrow$ discrete space: Similar to the time-dependent properties "discrete" and "continuous", a corresponding distinction can be drawn with respect to spatial state variables. If a model consist only of individual values that are spatially separated or independent of the location, the model will be referred to as discrete. If the state of a model is given at any point within a specific area, the model is continuous over space.

The model introduced in this chapter constitutes a deterministic, space-continuous and time-continuous model. But the model is not clearly classifiable with respect to the other attributes. For example, the first-order-subproblem of the model is an highly dynamic system, whereas the second-order subproblem describes only the stationary (time-invariant) flow of acoustic streaming in steady state. Furthermore it can be noted that, although the model is mainly constructed by the use of explanatory components, some aspects are modeled descriptively for reasons of simplicity. With respect to the model-parameters it should be mentioned that not all quantities are clearly predefined by direct measurements or physical estimates. In consideration of their physical limits, these parameters must therefore be specified in the course of the modeling-process.

Since a model normally represents the original system only in certain aspects, its range of validity is limited. Therefore, as already discussed in chapter 1, every model is based on a specific purpose for which the model has been developed. Each model should yield adequate answers with respect to questions resulting from its purpose, but the validity of the model regarding other questions must be doubted. Often, different questions need to be treated by different models.

Another aspect in modeling is the resultant complexity of the model. On the one hand a model should be as efficient and simple as possible. If a model is designed for universal purposes, the complexity and usually also the susceptibility to errors will increase. On the other hand the model should reflect the characteristic aspects as realistic as possible. Therefore it is not easy to decide whether certain simplifications are reasonable and whether a certain component is important or not.

This chapter deals with the establishment of an abstract mathematical model which describes the motions of the fluid as well as its interaction with the adjacent tissues. The basic simplifications and assumptions made by the modeling process are outlined in section 2.1. The differential equations that are used for representing the fluid are established in section 2.2. In this context, a perturbation technique is introduced by which the fluidic system can be separated into an acoustic subproblem and an acoustic streaming subproblem. 
The first order system is presented in section 2.3 and describes the linear acoustic reaction of the fluid (the acoustic subproblem), the passive mechanics of the cochlear partition as well as the motility of the outer hair cells. The acoustically driven flows are approximated by the time-averaged second order system (the acoustic streaming subproblem) which is deduced in section 2.4.

\subsection{Simplifying Assumptions}

In order to obtain an implementable model of the cochlea some simplifications and assumptions are made while maintaining fundamental aspects to get a valid representation for estimating the occurrence and structure of acoustically driven flows within the fluidfilled chambers of the inner ear. For reasons of clarification, the direction that is associated with the spiral axis of the coiled cochlea is referred to as longitudinal. The plane perpendicular to the longitudinal axis is referred to as transversal. The direction of the basilar membrane fibres within this transversal plane is denoted as radial. The term vertical is associated with the direction that is perpendicular to the longitudinal and the radial orientations.

Dimension The choice of the dimension is a crucial aspect in modeling. For a wide range of applications, a three-dimensional model seems to be the most suitable for representing the reality. In contrast to this, a considerable effort would be required, particularly regarding the development, implementation and execution of a three-dimensional model. Three-dimensional models of the cochlea were, for instance, realized by Givelberg and Bunn [17] who used the immersed boundary method, as well as by Cheng et al. [11] and Böhnke and Arnold [5] who performed finite element simulations. However, the occurrence of acoustically driven flows could not be investigated by these models, due to simplifying assumptions made by the modeling process. The present work ignores the variations parallel to the radial width of the basilar membrane. Therefore, the numerical simulations presented in this work are based on a two-dimensional model. Nevertheless, for a more detailed examination of acoustically driven flows, a generalization to three dimensions would be desirable.

Geometry Due to the reduction of the dimension, the spiral coiling of the cochlea can not be taken into account. It is assumed that the main cause of this snail shell shape of a mammalian cochlea lies in a better utilization of the space and a more efficient accessibility of blood vessels and nerve fibers (cf. [50]). Recently, a strong correlation between the change of longitudinal curvature and the audible range for different mammalian species suggest that the spiral coiling also has a certain influence on the low-frequency hearing limit. It is assumed that this effect is induced by a stronger focus of acoustic energy at the outer boundaries, particularly at more apical sites where the curvature is higher (cf. [29]). Altogether, the spiral coiling appears to play only a subordinate role and therefore it seems to be reasonable to neglect the longitudinal curvature in order to analyze acoustically 


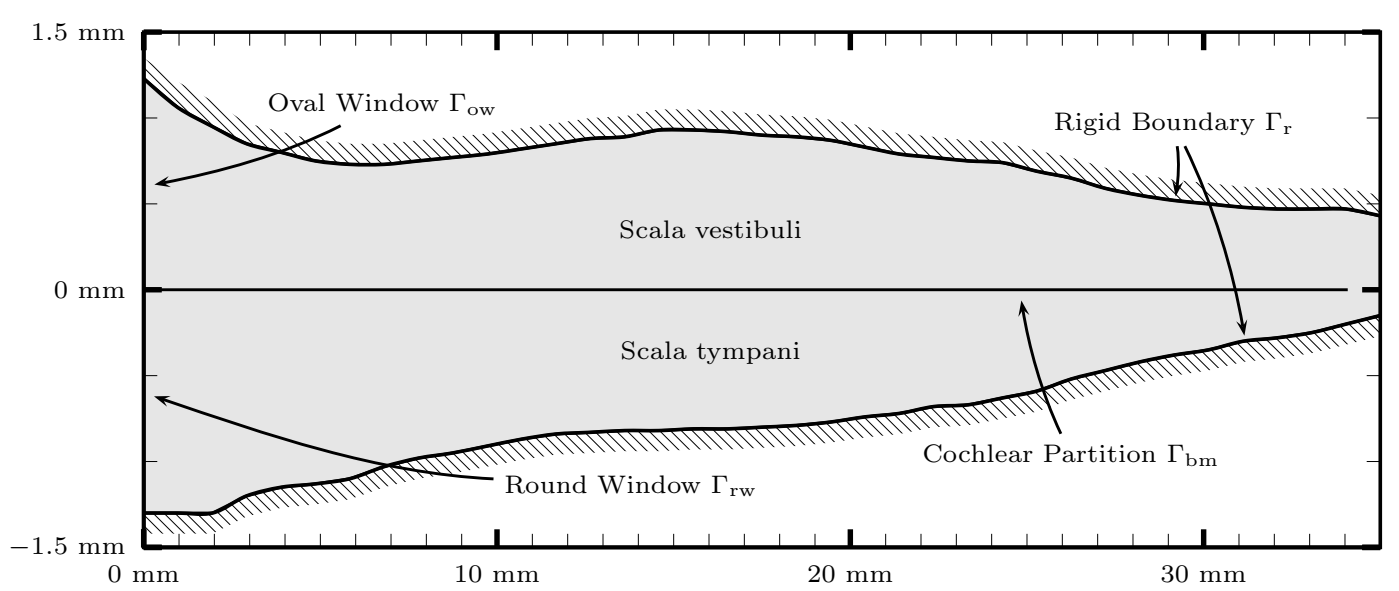

Figure 2.1: The two-dimensional shape of the uncoiled human cochlea model. The heights of the scala vestibuli and scala tympani are taken from Wysocki [52]. The scala vestibuli and the scala tympani constitute the fluid domain $\Omega$ and these chambers are bordered by the rigid boundary $\Gamma_{\mathrm{r}}$, the oval window $\Gamma_{\mathrm{ow}}$ and the round window $\Gamma_{\mathrm{rw}}$. Both ducts are separated by the cochlear partition $\Gamma_{\mathrm{bm}}$.

driven flows. As it can be seen in figure 2.1 the coiled tube of the cochlea is represented by an elongated flat shape. The height of the scala vestibuli and scala tympani are adapted from Wysocki [52], who studied the the dimensions of the chambers on the basis of 25 human cadavers.

Boundaries The boundary of the cochlea model can be divided into the rigid boundary $\Gamma_{\mathrm{r}}$, the oval window $\Gamma_{\mathrm{ow}}$ and the round window $\Gamma_{\mathrm{rw}}$ (cf. figure 2.1). The oval window consists of the stapes which act as an acoustic radiator in order to stimulate the cochlear system. The piston-like motions of the stapes can be represented by corresponding displacements of the associated boundary. The round window is closed by a flexible membrane which allows the fluid to move within the cochlea to a certain extent. For reasons of simplification, the functionality of the round window membrane is imitated by using a specific boundary condition as described in section 2.2.3.

Cochlear Partition To further reduce the complexity of the model, some anatomical details of the interior structures are also neglected. The Reissner's membrane is a very thin (only two-cell layer thick) partition which separates the scala tympani from the scala media. It is assumed that the Reissner's membrane acts not as a barrier of acoustic stimuli, but rather as a separation between the perilymph and the endolymph in order to preserve the electrical potential difference between both fluids. (cf. [49]) Therefore, the basilar membrane in combination with the scala media and its interior structures (e.g. the tectorial membrane, organ of corti, hair cells) are represented by the common onedimensional domain $\Gamma_{\mathrm{bm}}$. Furthermore, it is assumed that the cochlear partition can be modeled as a continuum and that it can only be deflected in vertical direction. Although the radial width of the basilar membrane cannot be taken directly into account by a 


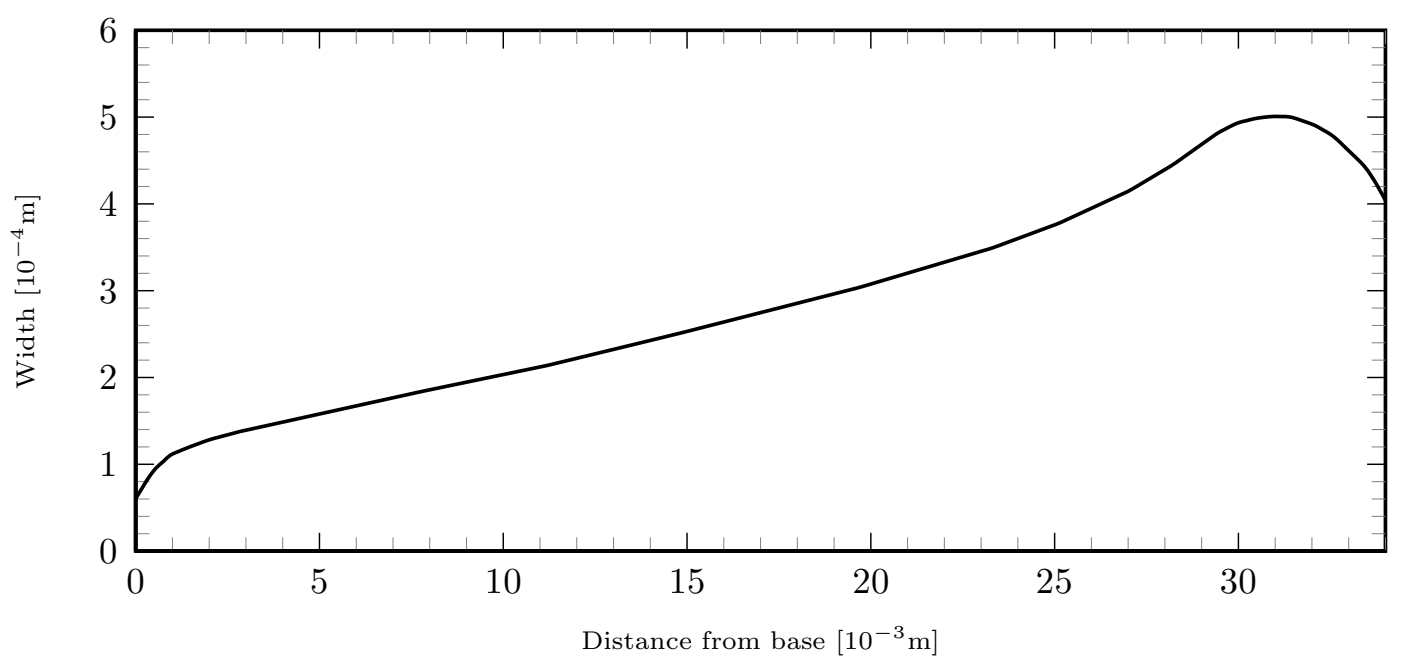

Figure 2.2: Width of the basilar membrane. The data is taken from Nobili [31].

two-dimensional representation of the cochlea, it is well accepted that the width plays an important role within the mechanics of hearing. Therefore, the influence of this width is considered indirectly by the system of equations as described in chapter 2.3.2. According to Nobili [31], the width of the basilar membrane is chosen as it can be seen in figure 2.2.

Fluid The scala vestibuli as well as the scala tympani are filled with a watery fluid (perilymph). Both chambers are connected by the helicotrema at the apex and constitute the fluid domain $\Omega$. In the course of the numerical simulation of the fluid, it is assumed that the continuum hypotheses can be applied. Since the exact specifications of this fluid are not known, the perilymph is, similar to the properties of water, assumed to be a slightly compressible, linearly bulk- and shear-viscous fluid.

\subsection{The Fluid Dynamics}

Fluid dynamics is a discipline of natural sciences and a branch of continuum mechanics. It deals on the one hand with the motions of a fluid in space and time and on the other hand with the various and mutual forces within the fluid causing the motions.

Due to the molecular structure, a fluid is neither fully homogeneous nor isotropic. For an exact specification of the dynamics of a fluid, it would be necessary to consider the mutual interactions of individual molecules and atoms. In principle, it is quite possible (and sometimes it is also necessary) to model the fluid dynamics on an atomic level to understand and simulate a variety of phenomena. But often, the exact physical behaviour of individual particles is not relevant and the scale of a typical length in the system is large compared to the length scales of molecular structures. In this case one can take advantage of the large number of particles within a fluid. By averaging physical quantities 
over space and time in an appropriate way, an hypothetical model can be constructed. In such a model all kinematic and kinetic quantities can be specified at infinitesimally small points. Furthermore, it is assumed that all these quantities vary continuously so that their spatial derivatives are also continuous. If a model describes a physical matter as such a continuous matter the model will be termed as continuum. For a wide range of applications a representation of a matter as a continuum is well suited.

For the mathematical formulation of the dynamics of a continuum, two different kinds of descriptions have been established. In the Lagrangian specification physical properties are defined as a function of individual material points and a time parameter. The Lagrangian specification is usually used for the description of solids, since the primary interest is the evolution of its current configuration and the analysis of physical quantities at specific locations of the material. In contrast to the Lagrangian specification, the idea of the Eulerian specification is to describe physical quantities at specific locations $\boldsymbol{x}$ in space, regardless of the material particles at these places. This kind of description is normally preferred in the context of the analysis of fluid flows due to potentially large changes of the material configuration.

\subsubsection{Conservation Equations}

In continuum mechanics the balance equations are the basis for the description of an arbitrary material, no matter whether it is a solid, a liquid or a completely different material. The conservation of mass equation (which is also referred to as continuity equation) can be written as

$$
\frac{\partial \rho}{\partial t}=-\operatorname{div}(\rho \boldsymbol{v})
$$

where $\rho(\boldsymbol{x}, t)$ denotes the scalar density field, $\boldsymbol{v}(\boldsymbol{x}, t)$ the vectorial field of the velocity and $t$ the time-parameter. In principle, the continuity equation states that the time rate of change of the total mass within an arbitrarily chosen spatial volume must be equal to the inflow of mass through the borders of the volume. In a similar way, the balance of linear momentum can be expressed in terms of the stress tensor $\boldsymbol{\sigma}$ by which the force that acts on the surface of a spatial region can be determined. If external volume forces (e.g. gravitation) are neglected, the forces acting through the volume surface must be identical to the time rate of change of the total linear momentum within the volume. Therefore, the conservation law of linear momentum can be written as

$$
\rho\left(\frac{\partial \boldsymbol{v}}{\partial t}+(\operatorname{grad} \boldsymbol{v}) \cdot \boldsymbol{v}\right)=\operatorname{div} \boldsymbol{\sigma} .
$$

Both conservation equations are formulated on the basis of the Eulerian specification. Furthermore, it was shown (e.g. [36]) that the principle of the balance of angular momentum results in the relation

$$
\sigma_{i j}=\sigma_{j i}
$$




\begin{tabular}{llll}
\hline Symbol & Value & Name & Unit \\
\hline$\rho^{(0)}$ & 998.0 & ambient density & $\mathrm{kg} / \mathrm{m}^{3}$ \\
$c_{0}$ & 1484.0 & small signal sound speed & $\mathrm{m} / \mathrm{s}$ \\
$\zeta$ & $3.0 \times 10^{-3}$ & bulk viscosity & $\mathrm{N} \mathrm{s} / \mathrm{m}^{2}$ \\
$\eta$ & $1.0 \times 10^{-3}$ & shear viscosity & $\mathrm{N} \mathrm{s} / \mathrm{m}^{2}$ \\
\hline
\end{tabular}

Table 2.1: Constants that are needed for the simulation of the fluid dynamics.

This means that the stress tensor must be symmetric.

A continuum-mechanical system will usually include an additional conservation equation that also takes thermodynamical effects into account. In this context, either the internal energy or the entropy of the continuum has to be considered as an additional unknown function. In this work it is assumed that these thermodynamical effects have no significant influence which means that potential energy fluctuations do not change the velocity-, density- or pressure field considerably. Therefore, the mechanical system can be decoupled from the balance law of energy or entropy and its associated fields do not have to be explicitly taken into account by the simulation process. (cf. [47])

\subsubsection{Constitutive Relations}

The conservation laws of mass, linear momentum and angular momentum constitute a system of 7 equations that contains 13 unknown functions $\left(v_{i}, \rho, \sigma_{i j}\right)$. In order to identify a unique solution of these different field functions the system of equations must be supplemented by further relations that are usually referred to as constitutive relations. In contrast to the conservation equations, which are fundamental principles of physics and which are applicable for all kinds of materials, the constitutive relations are normally deduced from experimental observations and may differ from material to material. Normally, the development of such constitutive relations is accompanied with an idealization of the reality.

In contrast to solids, fluids are normally characterized by the relative motility of its molecules. Or more precisely, a fluid at rest cannot preserve shear stresses (cf. [19]). Therefore, it has been proven useful to split the stress tensor into an hydrostatic stress tensor and a stress deviator tensor with zero trace. In doing this, the stress tensor can be written as

$$
\boldsymbol{\sigma}=\frac{1}{3} \operatorname{tr} \boldsymbol{\sigma} \boldsymbol{I}+\boldsymbol{s}=-p \boldsymbol{I}+\boldsymbol{s} .
$$

The scalar function $p(\boldsymbol{x}, t)$ denotes the pressure field. It turned out that the dynamics of a variety of different fluids and gases for a wide range of applications can be modeled very well if a linear relationship between the deviatoric stress and the strain rate is assumed. In this context, the local strain rate of a material can be described in terms of the velocity by the tensor (cf. e.g. [19])

$$
\boldsymbol{D}:=\frac{1}{2}\left(\operatorname{grad} \boldsymbol{v}+(\operatorname{grad} \boldsymbol{v})^{T}\right) .
$$


For the specification of the constitutive relationship, the linear relationship should be in conformity with some basic assumptions of material theory. According to [47] and [19], the following items should, inter alia, be taken into account:

- Although fluids are in principle not isometric at the atomic level, an identical behavior of the hydrodynamic properties with respect to all directions can be usually observed at the macro-mechanical level.

- A physical quantity at a specific location is assumed to depend only on the physical properties of its local neighborhood.

- The dynamics of the fluid are only affected by its current and preceding configurations. This means that the system is deterministic. In most cases, it is sufficient to limit this kind of "memory" by taking only the current rate of change into account.

- The principle of material frame indifference states that the choice of the reference frame for describing the constitutive relations should have no influence on fluid dynamics.

As a consequence of these basic assumptions, it was shown (cf. [36]) that the linear relationship between the deviatoric stress tensor and the strain rate tensor can be written - in its most general form - as

$$
\boldsymbol{s}=\lambda \operatorname{tr}(\boldsymbol{D}) \boldsymbol{I}+2 \eta \boldsymbol{D}
$$

where $\lambda$ and $\eta$ constitutes appropriate constants of proportionality. The constant $\lambda$ is usually replaced by the expression $\zeta-\frac{2}{3} \eta$ in order to establish material coefficients that are associated with either the isotropic variation or the deviatoric variation. Due to their physical interpretation, the coefficients $\eta$ and $\zeta$ are then referred to as shear viscosity and bulk viscosity. Using 2.4 and 2.6, the stress tensor can be evaluated to

$$
\boldsymbol{\sigma}=-p \boldsymbol{I}+\left(\zeta-\frac{2}{3} \eta\right) \operatorname{tr}(\boldsymbol{D}) \boldsymbol{I}+2 \eta \boldsymbol{D} .
$$

Now, the number of unknown functions can be reduced by inserting this explicit definition of the stress tensor into the balance of momentum equation 2.2. In doing so, the divergence of the stress tensor can be determined according to the specification of the strain rate tensor 2.5 to

$$
\operatorname{div} \boldsymbol{\sigma}=-\operatorname{grad} p+\left(\zeta+\frac{\eta}{3}\right) \operatorname{grad} \operatorname{div} \boldsymbol{v}+\eta \Delta \boldsymbol{v} .
$$

Due to the usage of the pressure field as an additional unknown scalar function, another constitutive relation is needed. Since no thermal effects are considered by the modeling process, it is assumed that the pressure depends solely on the density and is given by a thermal equation of state $p=p(\rho)$. In acoustics, the deviations of the pressure and the density from its respective ambient values can be approximated by the linear relationship

$$
p-p^{(0)}=\left.\frac{\partial p}{\partial \rho}\right|_{\rho^{(0)}}\left(\rho-\rho^{(0)}\right)=c_{0}^{2}\left(\rho-\rho^{(0)}\right)
$$


where the constant of proportionality can be identified as the square of the so-called small signal sound speed $c_{0}$.

\subsubsection{Boundary Conditions}

In this work, it is assumed that the velocity of the viscous perilymph vanishes relative to the solid boundaries. This specification is well known as no-slip-condition. By the use of the Eulerian specification, the no-slip condition at the rigid boundaries can be simply stated as

$$
\boldsymbol{v}(\boldsymbol{x}, t)=\mathbf{0} \quad \text { for all } \boldsymbol{x} \in \Gamma_{\mathrm{r}} .
$$

With regard to moving boundaries, the no-slip condition implies that the fluid-motion is equal to the associated motion of the boundary. Thus, if $\boldsymbol{\zeta}\left(\boldsymbol{x}_{0}, t\right)$ denotes an arbitrary displacement of the boundary which is deflected out of its resting position $\boldsymbol{x}_{0}$, the velocity at the displaced position can be evaluated to

$$
\boldsymbol{v}\left(\boldsymbol{x}_{0}+\boldsymbol{\zeta}\left(\boldsymbol{x}_{0}, t\right), t\right)=\frac{\partial \boldsymbol{\zeta}\left(\boldsymbol{x}_{0}, t\right)}{\partial t}
$$

For the purpose of simplification explicit changes of the domain, caused by the displacements of the moving boundaries, are neglected. This simplification is reasonable and possible, since the oscillations of the boundaries are substantially smaller than the proportions of the cochlea model. Equation 2.11 constitutes a Lagrangian specification of the velocity at the boundary. For the adaption of the boundary condition to an Eulerian specification, a vectorial Taylor expansion of the left hand side can be performed to obtain

$$
\boldsymbol{v}\left(\boldsymbol{x}_{0}, t\right)=\frac{\partial \boldsymbol{\zeta}}{\partial t}-\left(\operatorname{grad} \boldsymbol{v}\left(\boldsymbol{x}_{0}, t\right)\right) \cdot \boldsymbol{\zeta}-\ldots
$$

If $\xi$ denotes the vertical displacement of the basilar membrane and if $\xi_{\text {ow }}$ represents the horizontal displacement of the stapes within the oval window, the boundary condition 2.12 can be simply concretized by using the identities

$$
\begin{array}{ll}
\boldsymbol{\zeta}=\left(\begin{array}{ll}
0 & \xi
\end{array}\right)^{T} & \text { for all } \boldsymbol{x} \in \Gamma_{\mathrm{bm}+} \cup \Gamma_{\mathrm{bm}-}, \\
\boldsymbol{\zeta}=\left(\begin{array}{ll}
\xi_{\text {ow }} & 0
\end{array}\right)^{T} & \text { for all } \boldsymbol{x} \in \Gamma_{\mathrm{ow}} .
\end{array}
$$

In contrast to the coupling between the fluid and the basilar membrane, it is assumed that the round window has no significant influence on the fluid in the case of usual acoustic stimulations. Therefore, a fluid-structure-interaction with the round window is not directly taken into account by the modeling process presented in this work. On the one hand the membrane within the round window prevents the leakage of fluid, on the other hand it is flexible enough to allow free movements of the fluid up to a certain extent. This behavior can be imitated if the boundary pretends to be non-existent with respect to the longitudinal velocity component. In other words, no explicit boundary condition is applied on the first velocity component at the round window. 
Furthermore, it is assumed that no external forces act on the fluid across the outer boundaries. In terms of the stress tensor $\boldsymbol{\sigma}$, this condition can be written as

$$
\boldsymbol{\sigma}(\boldsymbol{x}, t) \cdot \boldsymbol{n}(\boldsymbol{x}, t)=0 \quad \text { for all } \boldsymbol{x} \in \Gamma
$$

where $\boldsymbol{n}$ indicates the (unit) outward normal vector with respect to the boundary of the fluid volume. At the beginning of the simulation process $t_{0}$, it is presumed that the system is at rest. As a consequence, the velocity field as well as the displacement variables are set to zero. The pressure and the density field are predefined by its constant ambient values $p^{(0)}$ and $\rho^{(0)}$. Formally, the initial conditions can be written as

$$
\begin{array}{ll}
\boldsymbol{v}\left(\boldsymbol{x}, t_{0}\right)=\mathbf{0} & \text { for all } \boldsymbol{x} \in \Omega, \\
p\left(\boldsymbol{x}, t_{0}\right)=p^{(0)} & \text { for all } \boldsymbol{x} \in \Omega, \\
\rho\left(\boldsymbol{x}, t_{0}\right)=\rho^{(0)} & \text { for all } \boldsymbol{x} \in \Omega, \\
\xi\left(\boldsymbol{x}, t_{0}\right)=0 & \text { for all } \boldsymbol{x} \in \Gamma_{\mathrm{bm}}, \\
\xi_{\text {ow }}\left(\boldsymbol{x}, t_{0}\right)=0 & \text { for all } \boldsymbol{x} \in \Gamma_{\mathrm{ow}} .
\end{array}
$$

\subsubsection{Perturbation Expansion}

As mentioned above, acoustic streaming is a nonlinear phenomenon caused by the nonlinear terms within the governing equations of the fluidic system. A direct numerical simulation of the fully nonlinear Navier-Stokes-Equations in order to analyze acoustic streaming was performed, for instance, by Yano [53] or Boluriaan and Morris [7]. In contrast to the mean flows expected within the cochlea, their models aim to simulate acoustic streaming that is characterized by an high Reynolds number. In this context, the Reynolds number is a measure that quantifies the relative importance of inertial forces in relation to viscous forces with respect to the mean flows. In the scope of low Reynolds numbers, the use of a perturbation technique has advantages over the fully nonlinear approach, since the perturbation approach represents the nonlinear problem by a set of linear sub-problems. Normally, it is sufficient to consider only the first two linear sub-problems. This perturbation method has already been successfully adopted by several authors (e.g. [24, 9, 26]).

For the implementation of the perturbation approach, the unknown functions are represented by Taylor expansions in the small Mach number $\epsilon \ll 1$, which is defined as the ratio between a typical velocity of the fluid particles and the small signal sound speed $c_{0}$. The Taylor series of the velocity-field is, for instance, given by

$$
\boldsymbol{v}(\boldsymbol{x}, t, \epsilon)=\underbrace{\left.\boldsymbol{v}(\boldsymbol{x}, t, \epsilon)\right|_{\epsilon=0}}_{=: \boldsymbol{v}^{(0)}}+\underbrace{\left.\frac{\partial \boldsymbol{v}(\boldsymbol{x}, t, \epsilon)}{\partial \epsilon}\right|_{\epsilon=0}}_{=: \boldsymbol{v}^{(1)}} \epsilon+\underbrace{\left.\frac{\partial^{2} \boldsymbol{v}(\boldsymbol{x}, t, \epsilon)}{\partial \epsilon^{2}}\right|_{\epsilon=0}}_{=: \boldsymbol{v}^{(2)}} \epsilon^{2}+\mathcal{O}\left(\epsilon^{3}\right) .
$$

Similarly, the other field-functions (pressure, density,...) can also be represented by such a perturbation expansion in $\epsilon$. This approach is, of course, only applicable under the 
assumption that the perturbed problem arises smoothly from an initial problem through the continuous variation of the parameter $\epsilon$. In this context, the initial problem is characterized by a zero Mach number, which means that there will be no particle motions, provided that the sound speed remains constant. Then the system can be fully described by the ambient values $p^{(0)}$ and $\rho^{(0)}$.

Terms of higher order can now be determined by the use of a successive method. This procedure is based on the principle that terms of different order are independent from each other (cf. [14]). This means that after substituting the respective perturbation expansions for the unknown functions, each equation can be separated into a set of equations, whereby each relation consists only of terms of the same order.

Lighthill [26] pointed out that this separation of different terms should primarily depend on their numerical dimension and not on their mathematical order. He concluded that the resultant second order system (which is used for the evaluation of acoustic streaming) neglect a fourth order quantity that would take inertial effects of the mean flows into account. These inertial effects become more and more significant for applications that are characterized by an high Reynolds number. In these cases, the successive perturbation method would probably fail to approximate the mean flows correctly.

In steady state, the first order functions describe the harmonic excess values of the fundamental acoustic field with the angular frequency $\omega$, provided that the system is stimulated by a sinusoidal excitation of the same frequency. Then, the second order functions can be associated with the second order harmonic field of double the frequency as well as a second order steady streaming. (cf. [24, 9]) In this work, the main focus lies on the determination of this secondary steady flow field, since it constitutes a first order approximation of acoustic streaming. Any higher-order mean flows are neglected, due to the rapid decrease of their magnitudes. Therefore, only the first order approximation of the resultant mean flows is considered in this work.

The first order system of the perturbation approach (hereinafter also referred to as acoustic subproblem) is established in chapter 2.3.1. The acoustic streaming subproblem, which corresponds to the averaged second order system, is deduced in chapter 2.4.

\subsection{The First Order System}

On the basis of the perturbation theory, the nonlinear system of equations that describes the behavior of the fluid is separated into a first and a second order system. As mentioned above, the first order system describes the fundamental acoustic field which is mainly influenced by the fluid-structure-interaction with the structural components of the cochlear system (cf. figure 1.3). This section deals on the one hand with the derivation of the first order perturbation of the fluidic system (cf. chapter 2.3.1) and on the other with the mathematical modeling of the relevant mechanical and physiological properties that represents the dynamics of the basilar membrane (cf. chapter 2.3.2). 


\subsubsection{The Acoustic Subproblem}

After substituting the perturbation expansions (cf. equation 2.21) for the velocity-, pressureand density-functions and considering only the first order terms, the first order conservation principles of mass and momentum can be written as

$$
\begin{aligned}
& \frac{\partial \rho^{(1)}}{\partial t}=-\rho^{(0)} \operatorname{div} \boldsymbol{v}^{(1)}, \\
& \rho^{(0)} \frac{\partial \boldsymbol{v}^{(1)}}{\partial t}=\operatorname{div} \boldsymbol{\sigma}^{(1)} .
\end{aligned}
$$

According to equation 2.8, the divergence of the first order stress tensor is given by

$$
\operatorname{div} \boldsymbol{\sigma}^{(1)}=-\operatorname{grad} p^{(1)}+\left(\zeta+\frac{\eta}{3}\right) \operatorname{grad} \operatorname{div} \boldsymbol{v}^{(1)}+\eta \Delta \boldsymbol{v}^{(1)} .
$$

In the same way, also the thermal equation of state 2.9 can be transformed into a first order version which results in the simple relation

$$
p^{(1)}=c_{0}^{2} \rho^{(1)} .
$$

By combining the previous equations, the stress tensor as well as the first order density function can be eliminated. Then, the acoustic subproblem that describes the fundamental acoustic field within the fluid is given by the following system of equations:

$$
\begin{aligned}
\frac{1}{c_{0}^{2}} \frac{\partial p^{(1)}}{\partial t} & =-\rho^{(0)} \operatorname{div} \boldsymbol{v}^{(1)} \\
\rho^{(0)} \frac{\partial \boldsymbol{v}^{(1)}}{\partial t} & =-\operatorname{grad} p^{(1)}+\left(\zeta+\frac{\eta}{3}\right) \operatorname{grad} \operatorname{div} \boldsymbol{v}^{(1)}+\eta \Delta \boldsymbol{v}^{(1)} .
\end{aligned}
$$

Finally, the boundary conditions have to be adapted. The first order approximation of the Eulerian specification of the no-slip condition 2.12 can be evaluated by taking only the first order terms into account. Therefore, the first order velocity components at the basilar membrane and at the oval window can be written as

$$
\begin{aligned}
\boldsymbol{v}^{(1)}(\boldsymbol{x}, t) & =\left[\begin{array}{ll}
0 & \frac{\partial}{\partial t} \xi(\boldsymbol{x}, t)
\end{array}\right]^{T} & & \text { for all } \boldsymbol{x} \in \Gamma_{\mathrm{bm}}, \\
\boldsymbol{v}^{(1)}(\boldsymbol{x}, t) & =\left[\begin{array}{ll}
\frac{\partial}{\partial t} \xi_{\text {ow }}(\boldsymbol{x}, t) & 0
\end{array}\right]^{T} & & \text { for all } \boldsymbol{x} \in \Gamma_{\mathrm{ow}} .
\end{aligned}
$$

\subsubsection{The Passive Mechanics of the Cochlear Partition}

The interactions between the fluid and the basilar membrane have a significant influence on the sound field within the inner ear. Therefore, it is essential to take the mechanical behavior of the cochlear partition into account. In this context, an approach, developed 


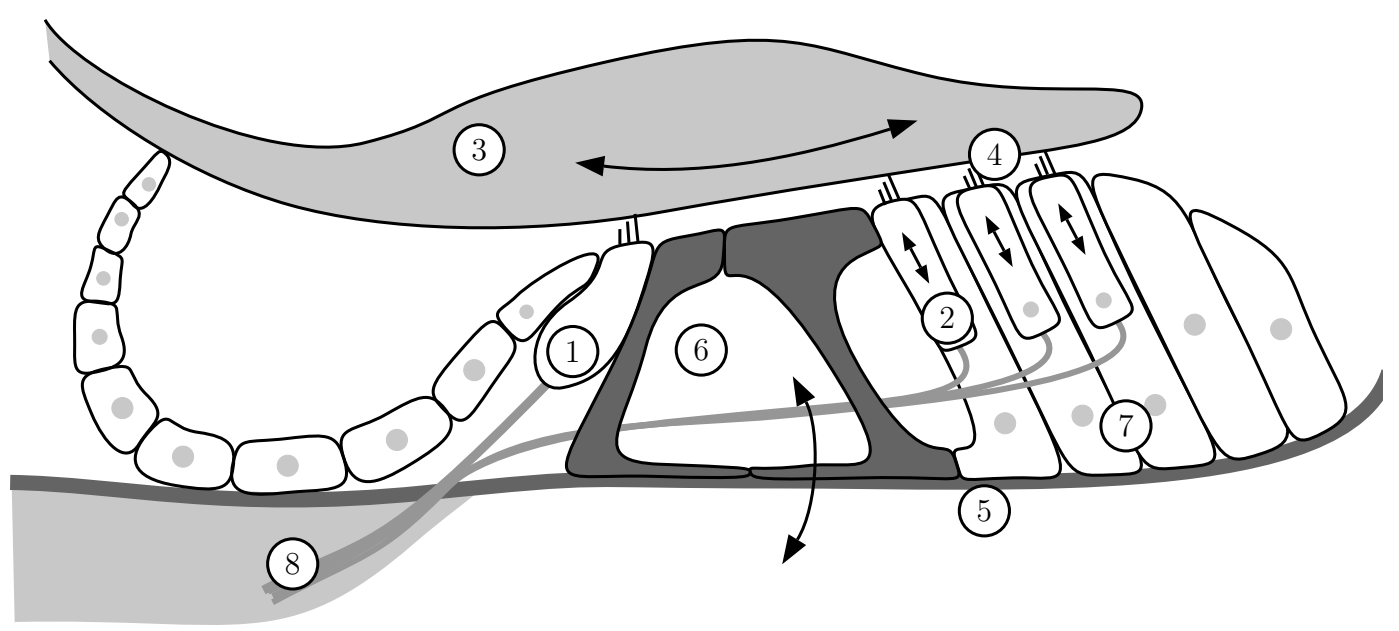

Figure 2.3: Cross section of a mammalian organ of corti: (1) Inner hair cell. (2) Outer hair cells. (3) Tectorial membrane. (4) Stereocilia. (5) Basilar membrane. (6) Tunnel of corti. (7) Cells of Deiters. (8) Nerve fibres. The arrows indicate the principal motions of the organ of corti. The pressure difference between the upper and lower chamber causes upward and downward movements of the basilar membrane. Furthermore, the tectorial membrane moves along the reticular lamina due to their different pivots.

by Mammano and Nobili [28, 33], is adapted that has proven to be a well-functioning onedimensional model for describing the deflections of the basilar membrane as cause of the pressure differences across the partition. In the first part of this section the main ideas for describing the basilar membrane as a linear system of damped oscillators are presented. The second part of this section focuses on the representation of an additional force term in order to model a physiologically mechanism, known as cochlear amplifier.

The idea that the inner ear may work as a frequency analyzer due to longitudinally varying physical properties of the organ of corti can be traced back to Helmholtz. He noted that the cochlear partition consists of an array of radially orientated fibres and that the length of these fibres increase from base to apex. Therefore, he hypothesized that these fibres may have different resonance frequencies at which they resonate. But it turned out that the damping of the surrounding fluid prevents a significant resonant behaviour of individual fibres. Nevertheless, the assumption that the cochlea acts principally as a frequency analyzer has proven to be true. (cf. [1])

Helmholtz idea that the cochlear partition can in principle be represented by a number of oscillators, which are characterized by different physical properties, is taken up by the model used here. Figure 2.4 shows a schematic illustration of such an array of oscillators. Each oscillator consists of a mass which can be set into vibration through a spring. The springs are characterized by its respective stiffness. Furthermore each spring-mass system is attached to a damper which tends to reduce the oscillation. But in contrast to such a discrete arrangement of individual oscillators, the basilar membrane is rather modeled as a continuum within the context of this work. According to Mammano and Nobili [28], such a damped oscillation of a continuous one-dimensional system that describes the vertical 


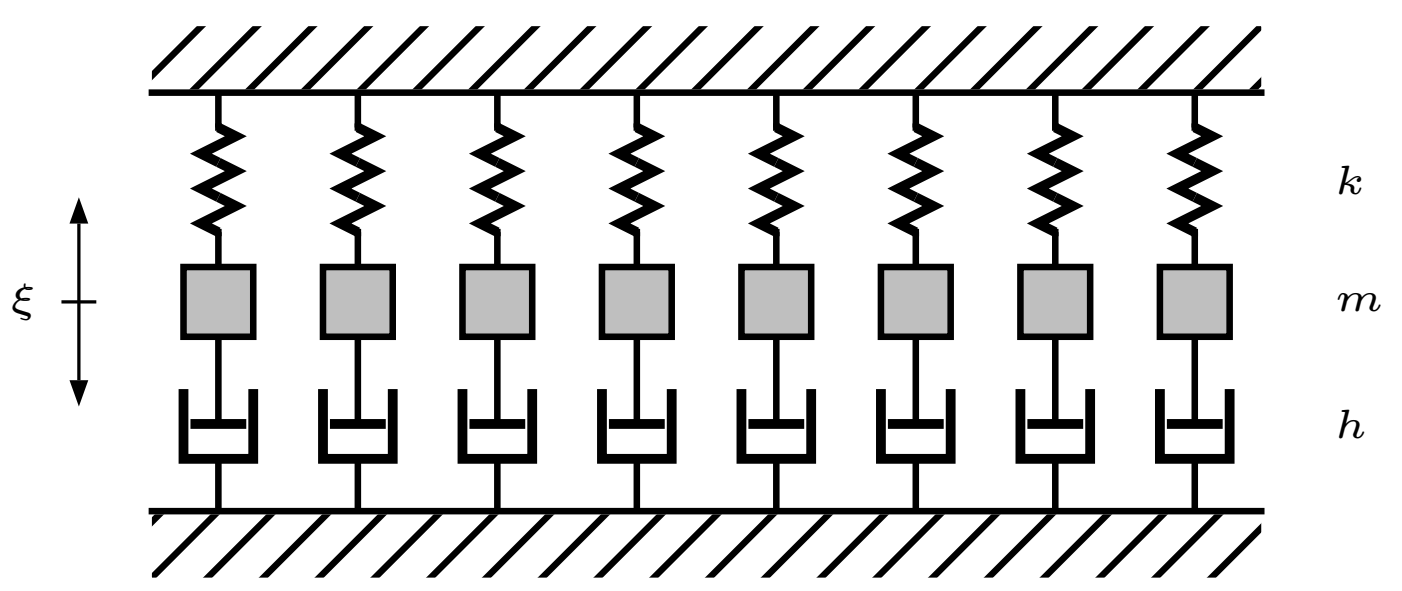

Figure 2.4: The mechanical properties of the basilar membrane can be represented by an array of damped oscillators. Each oscillator is characterized by its specific mass $m$, viscosity $h$ and stiffness $k$. Vertical displacements $\xi(x)$ are caused by external forces.

displacement of the basilar membrane $\xi(x, t)$ under the action of external forces can be represented by the differential equation

$$
m(x) \frac{\partial^{2} \xi(x, t)}{\partial t^{2}}+h(x) \frac{\partial \xi(x, t)}{\partial t}+k(x) \xi(x, t)=f_{p_{+}}(x, t)-f_{p_{-}}(x, t)+\vartheta(x, t)
$$

if it is assumed that all forces depend linearly on the displacement of the basilar membrane. The coefficient functions describe different physical properties of the basilar membrane. An adequate choice of these parameter functions is not easy, because the structure of the organ of corti is not homogeneous and the material properties are difficult to determine. Furthermore, it is not clear to what extent certain characteristics may be relevant for the oscillation process. However, in order to ensure that the coefficients range within the physical limits, they are estimated on the basis of some theoretical and dimensional considerations having regard to the biophysical structure of the organ of corti (cf. figure 2.8).

The first term on the left hand side of equation 2.30 is associated with the mechanical inertia of the system. The coefficient $m(x)$ describes the mass per unit length at the position $x$. According to a matlab-routine from Nobili [31, the mass is estimated by some dimensional considerations of the organ of corti. It is assumed that the organ of corti at the base has approximately a width of $50 \mu \mathrm{m}$ and a height of $40 \mu \mathrm{m}$ and that both properties increase exponentially up to four times from base to apex. An estimation of the effective mass per unit length at a specific location can be determined by the product of an average density of the tissue (which is assumed to be equal to the density of water), the height of the organ of corti and an effective width. This effective width is calculated as the geometric mean of the width of the organ of corti and the half of the width of the basilar membrane. The resultant mass per unit length is illustrated in figure 2.5.

The second term of the left hand side of equation 2.30 describes the damping of the 


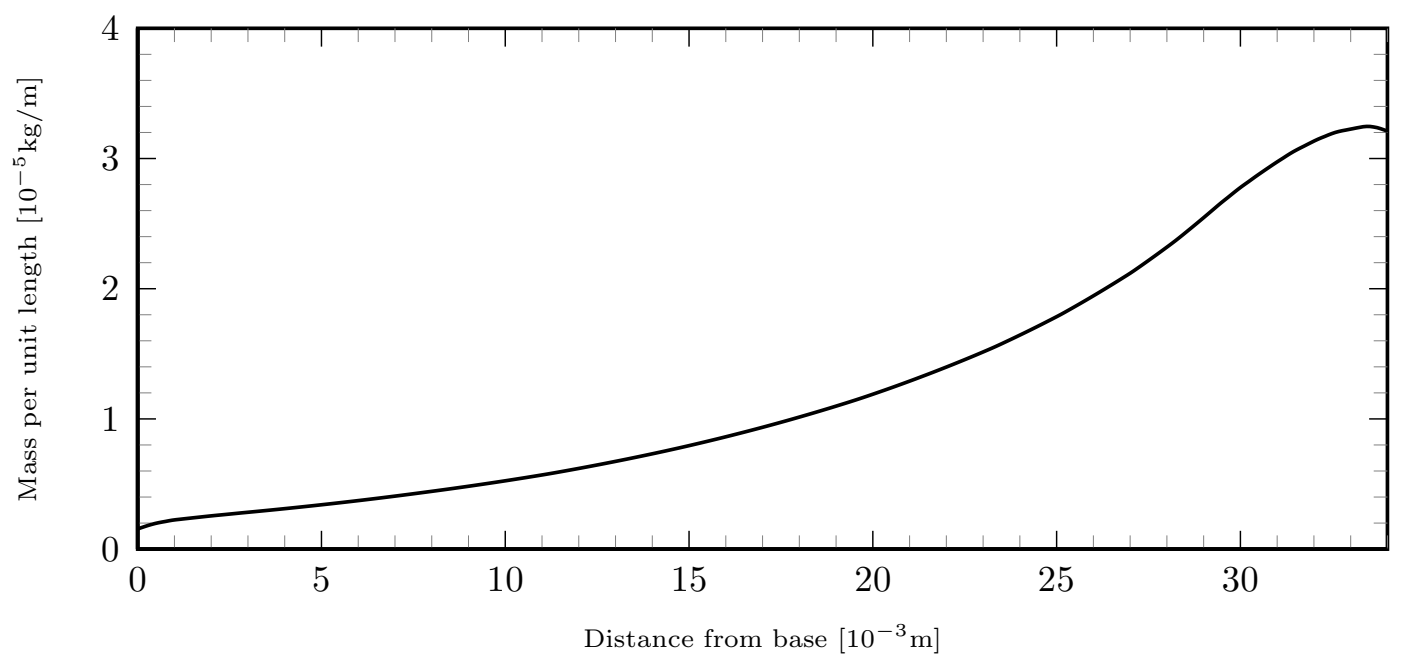

Figure 2.5: Effective mass per unit length of the basilar membrane that is assumed to be relevant for the inertial effects of the basilar membrane.

cochlear partition. In consideration of the realistic structure of the organ of corti, the displacement of the basilar membrane is characterized by a rotary motion of the tunnel of Corti as shown in figure 2.8. The reason for such a motion lies in the significant rigidity of the pillar cells. In contrast to the pillar cells, the outer hair cells and Deiters' cells can be much more easily deformed. As a result, the oscillation of the cochlear partition is probably accompanied by shearing motions of the outer hair cells and Deiters' cells. Within this work it is assumed that the damping is therefore mainly governed by the intrinsic viscosity of these cells. On the basis of the definition of shear viscosity within Newtonian fluids, the resistance per unit length can be roughly estimated by

$$
\eta_{\text {rad }} \frac{H_{\text {ohc }}(x)}{W_{\text {ohc }}(x)} \frac{\partial \xi}{\partial t}
$$

where $\eta_{\text {rad }}, W_{\text {ohc }}(x)$ and $H_{\text {ohc }}(x)$ are associated with the mean viscosity of the cells, the radial width and the transversal height of the effective segment at the longitudinal position $x$. According to [31], it is assumed that at the base of the cochlea the mean viscosity is approximately 30 times higher than the viscosity of water and that the effective ratio between the radial width and the transversal height is about 10. As it can be seen in figure 2.6](a), it is further assumed that the viscosity per unit length decreases about four times from base to apex.

For an infinitesimal small longitudinal segment, the associated resistance per unit length caused by the shear viscosity can be approximated - in a similar way as above - to

$$
\frac{\partial}{\partial x}\left(\eta_{\text {lon }} A(x) \frac{\partial^{2} \xi}{\partial x \partial t}\right)
$$




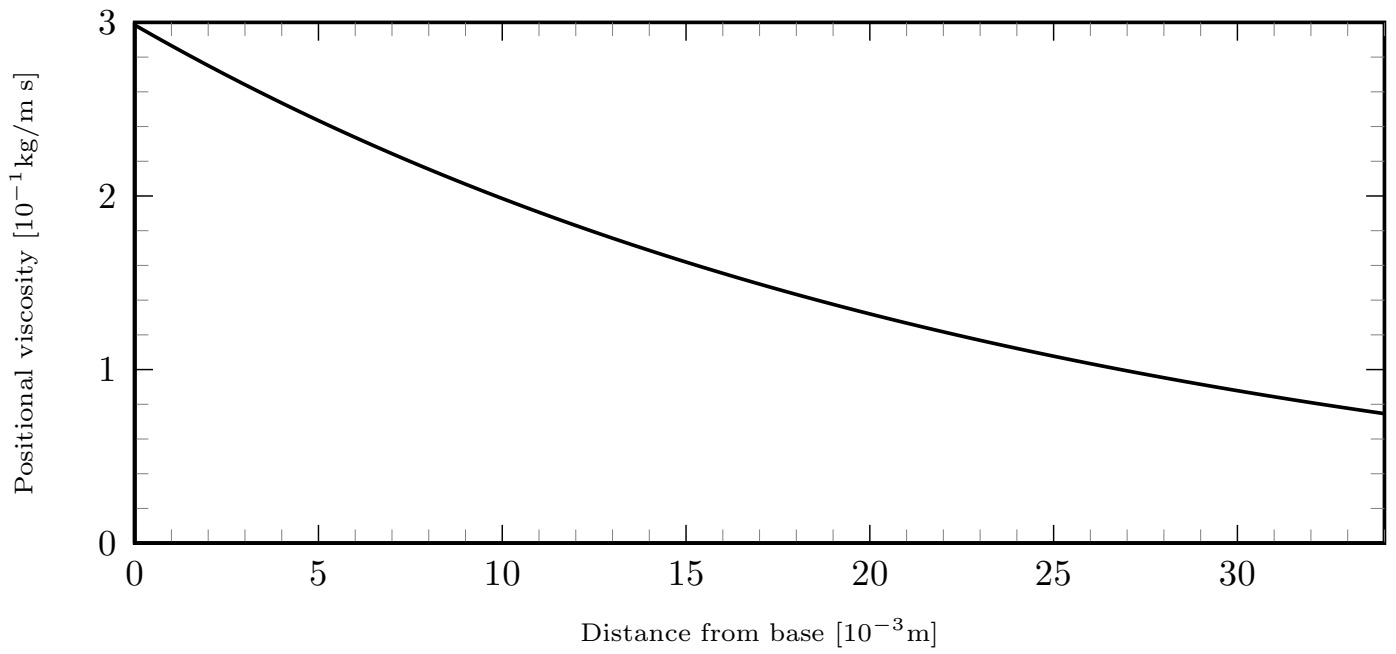

(a)

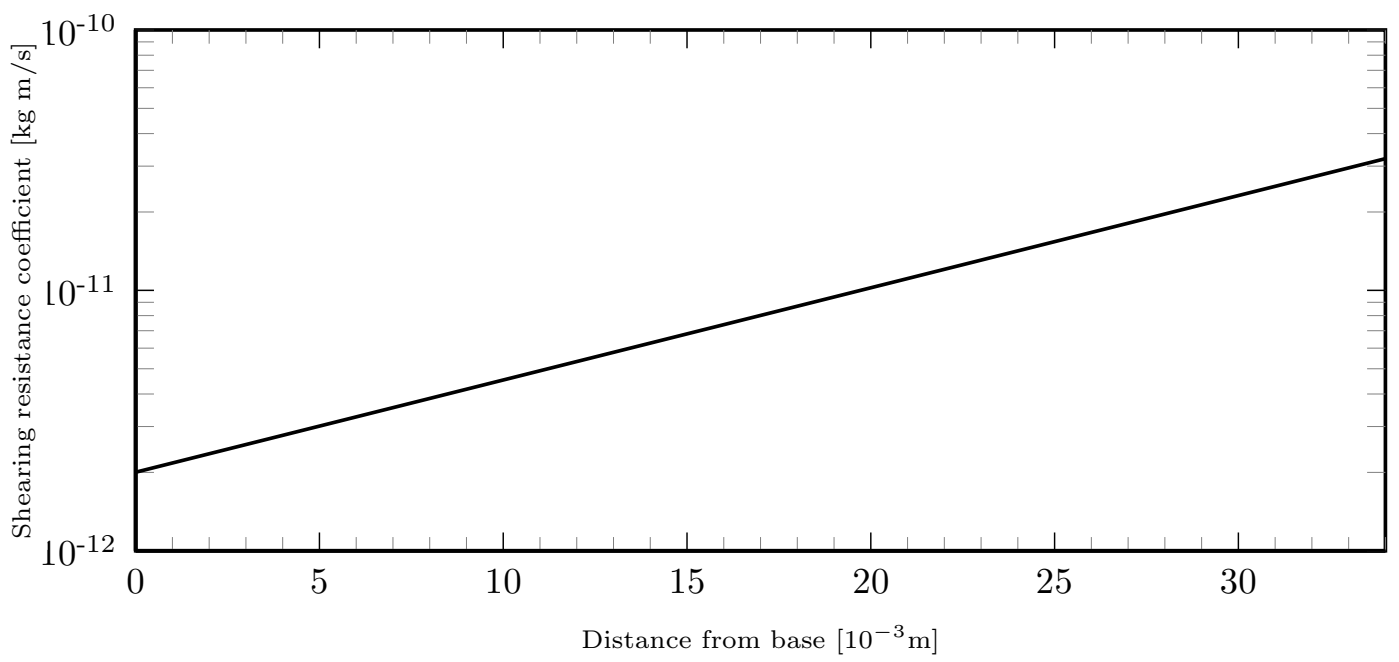

(b)

Figure 2.6: Damping properties of the basilar membrane. (a) The positional viscosity is specified by the vertical shearing motion of the segment consisting of the outer hair cells and the Deiters' cells along the radial direction. (b) The shearing resistance coefficient is associated with vertical shearing motions of the basilar membrane segments along the longitudinal axis. 


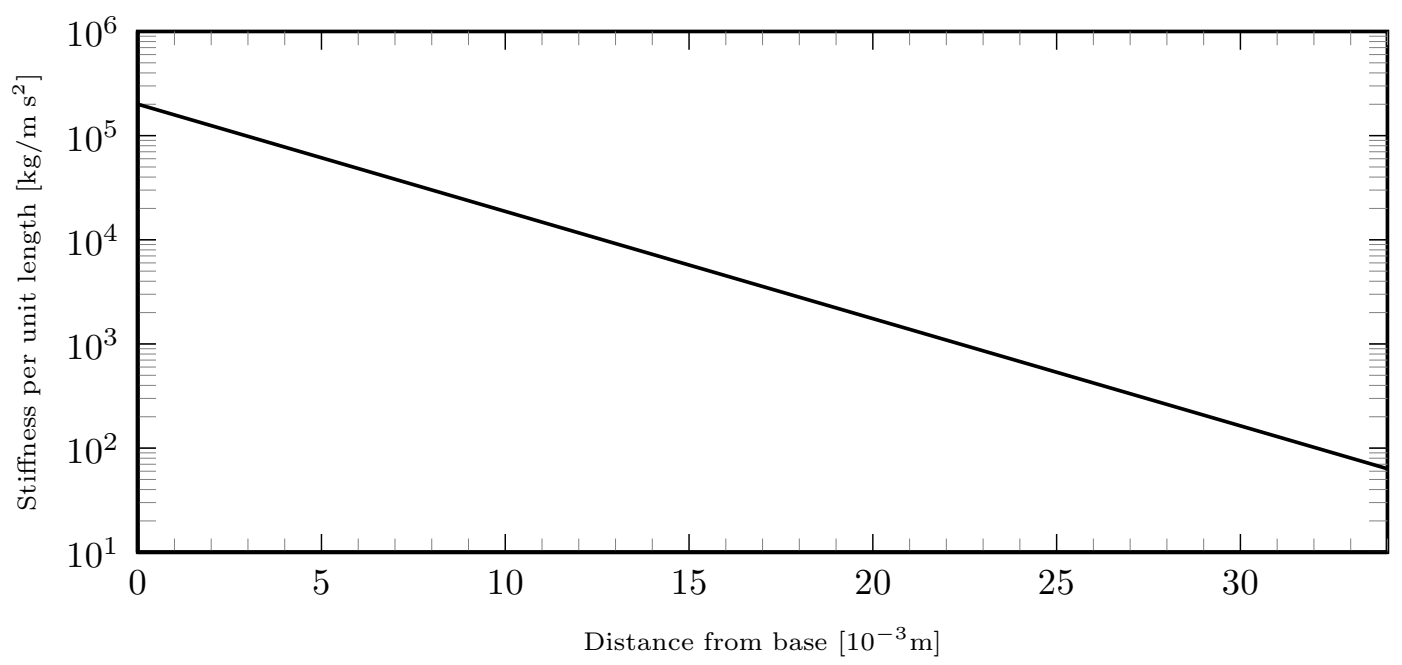

Figure 2.7: Virtual stiffness per unit length of the basilar membrane that is assumed to be relevant for the resistance caused by a displacement of the cochlear partition.

where $A(x)$ is the cross-sectional area of the organ of corti at the location $x$ and $\eta_{\text {lon }}$ a mean effective viscosity of the associated layer. As the spatial derivative of the velocity term indicates, a longitudinal shearing driven resistance can only occur if velocities of adjacent layers differ. On the basis of the same proportions of the organ of corti as already used for the estimation of the mass and under the assumption that the effective viscosity $\eta_{\text {lon }}$ is equal to the viscosity of water, the coefficient $\eta_{\mathrm{lon}} A(x)$ can be estimated as illustrated in figure 2.6](b). In comparison to the positional shearing driven resistance it turns out that the longitudinal coupling plays only a subordinate role and the longitudinal segments can oscillate almost independently from each other.

The last term of the left hand side of equation 2.30 represents the force per unit length applied by the stiffness of the cochlear partition. Measurements (e.g. [50]) indicate that the stiffness decreases by two to four orders of magnitude from base to apex. But, the physical dimensions of different measurements vary over a wide range. Furthermore, it is debatable whether these measurements are physiologically relevant within the working cochlea, since they are measured during displacements that are considerably larger than realistic displacements. (cf. [38]) Similar to [28], the stiffness is therefore chosen in such a way that the characteristic frequencies of the traveling wave roughly correspond to the (human) frequency-position map which was introduced by Greenwood [18] (cf. chapter 4.1.3). As a result of this procedure, the stiffness is set to $2 \times 10^{5} \mathrm{~kg} / \mathrm{m} \mathrm{s}^{2}$ at the base and decreases by 3.5 orders of magnitude from base to apex. (cf. figure 2.7)

The right hand side of equation 2.30 considers external forces acting on the basilar membrane. As a result of the fluid pressure above and below the basilar membrane, two hydrodynamic forces $f_{p_{+}}$and $f_{p_{-}}$occur. Since the pressure acts - at a specific location $x$ - on the whole width $w_{b m}(x)$ of the basilar membrane, the hydrodynamic forces may be 
written as

$$
f_{p_{ \pm}}(x, t)=\left.\frac{1}{2} w_{b m}(x) p^{(1)}(\boldsymbol{x}, t)\right|_{\boldsymbol{x}=(x, \pm 0)} .
$$

Due to the assignment of two different pressure values at the location $\boldsymbol{x}=(x, 0)$ (caused by the one-dimensional representation of the basilar membrane), the second component of the coordinates are additional labeled by a sign to indicate the location. Thus, a positive (negative) sign refers to the location that belongs to the upper (lower) duct. Actually, due to the anatomical structure and the lateral fixation of a basilar membrane segment (cf. figure 2.3), the pressure should not be accumulated uniformly along the radial width. This issue is taken into account (in a simplified manner) by introducing an effective width which is assumed to be half of the geometric width.

The force $\vartheta$ is associated to the internal amplification mechanism provided by the outer hair cells.

\subsubsection{Outer Hair Cell Motility}

The vertical motions of the basilar membrane are accompanied with a shearing displacement of the tectorial membrane in relation to the reticular lamina. This kind of motion is the result of different pivots of the tectorial membrane and the reticular lamina around which they rotate. (cf. [28]) The stereocilia of the outer hair cells are deflected by the shearing flow of the endolymph and due to a partial contact of the stereocilia with the bottom of the tectorial membrane. The deflection of these hair bundles causes in turn a change of the cell potential due to a mechanoelectrical transduction mechanism. If the stereocilia are deflected in the direction of its tallest outgrowth, the outer hair cell will be depolarized. Otherwise, a hyperpolarization is caused by a deflection in the opposite direction. Having regard to the anatomical structure of the cochlear cross-section a depolarization is the result of an upward motion of the basilar membrane (in the direction of the scala media). In contrast, a movement of the basilar membrane in the direction of the scala tympani brings about a hyperpolarization of the outer hair cells. As a result of electrical stimulation the outer hair cells are capable of changing its length. Therefore, the length change is often referred to as electromotility. The outer hair cell motility is mainly driven by a motor protein, which is known as prestin and which can be found in the lateral membrane of each cell. (cf. [1]) Measurements indicate (cf. [1]) that the length changes of outer hair cells are fast enough to have a mechanical effect on vibrations at acoustic frequencies. However, to this day there is some dispute about the exact internal mechanisms of the motility and the specific properties of the outer hair cells. Because this work is not primarily concerned with an exact representation of the complex processes of the outer hair cell motility, a simplified stimulus-response model (black box model) is adapted as it was suggested by Nobili et al. [33] in order to take the feedback from the outer hair cells into account. In this context, it is assumed that the relationship between the deflection $\zeta$ of the hair bundles and the force $\vartheta$, which is applied by the outer hair cells due to their 


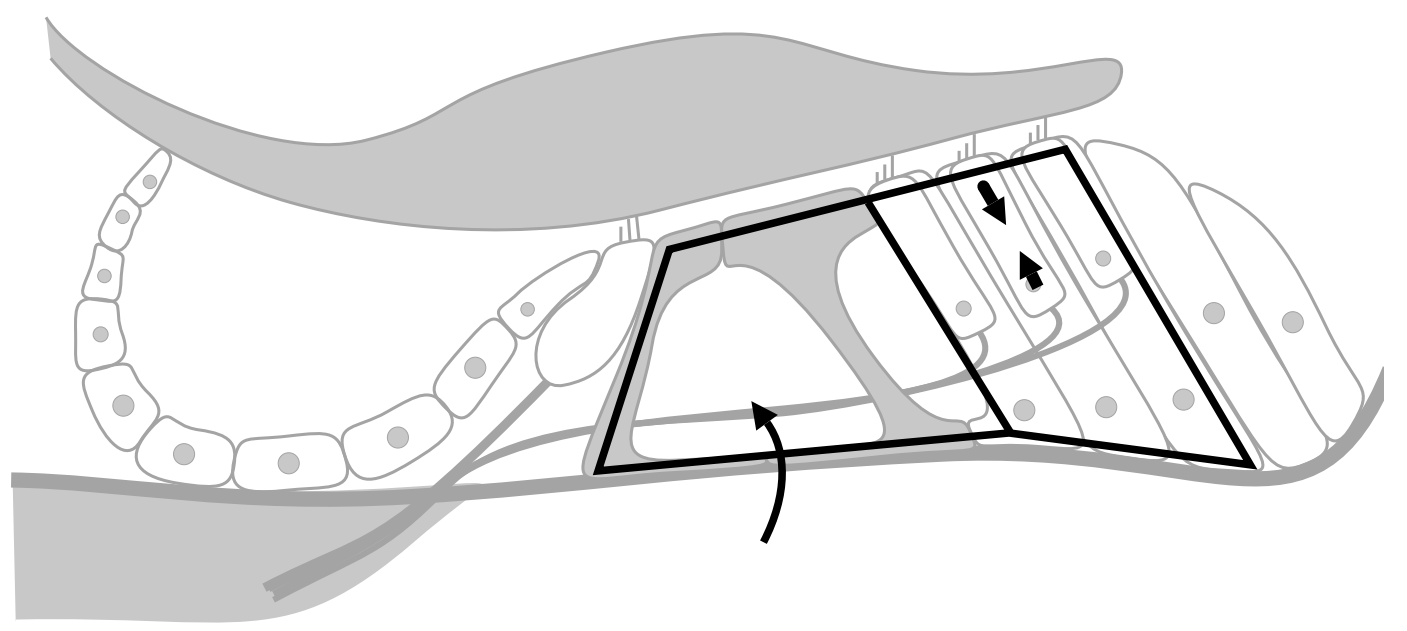

(a)

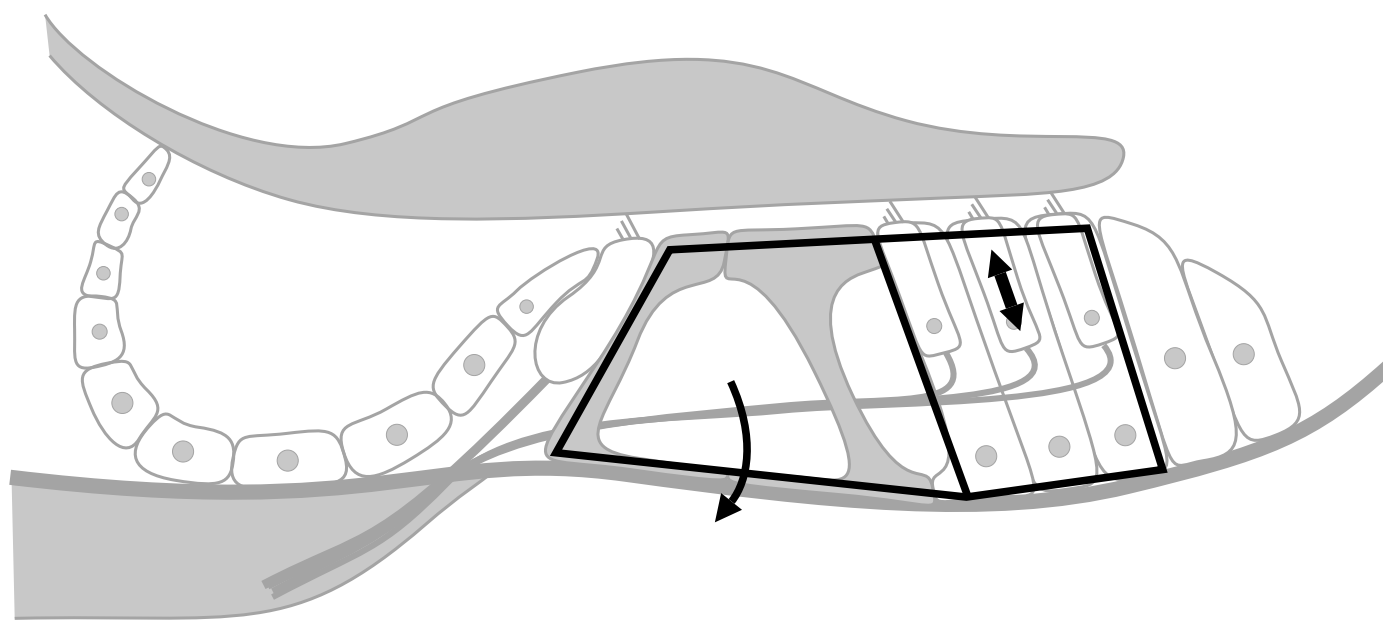

(b)

Figure 2.8: A displacement of the basilar membrane is accompanied with a rotational motion of the tunnel of corti around a pivotal point. Due to the rigidity of the pillar cells in combination with the reticular lamina the outer hair cells and Deiters' cells are deformed in order to compensate these motions. In this work, it is assumed that the internal shearing motion of the outer hair cells and the Deiters' cells respectively are mainly responsible for the damping effect. At the same time, the laminar shearing motion of the tectorial membrane relative to the reticular lamina causes a deflection of the stereocilia of the inner and outer hair cells. This parallel shift is the result of a different pivotal point around which the tectorial membrane rotates. A deflection of the hair bundles towards the largest one (which in principle can be associated with an upward displacement of the basilar membrane) causes in turn a contraction of the outer hair cells due to a mechanoelectrical transduction mechanism (cf. figure $(a)$ ). In contrast, an elongation of the outer hair cells is the result of a hair bundle deflection in the opposite direction (cf. figure (b)). 


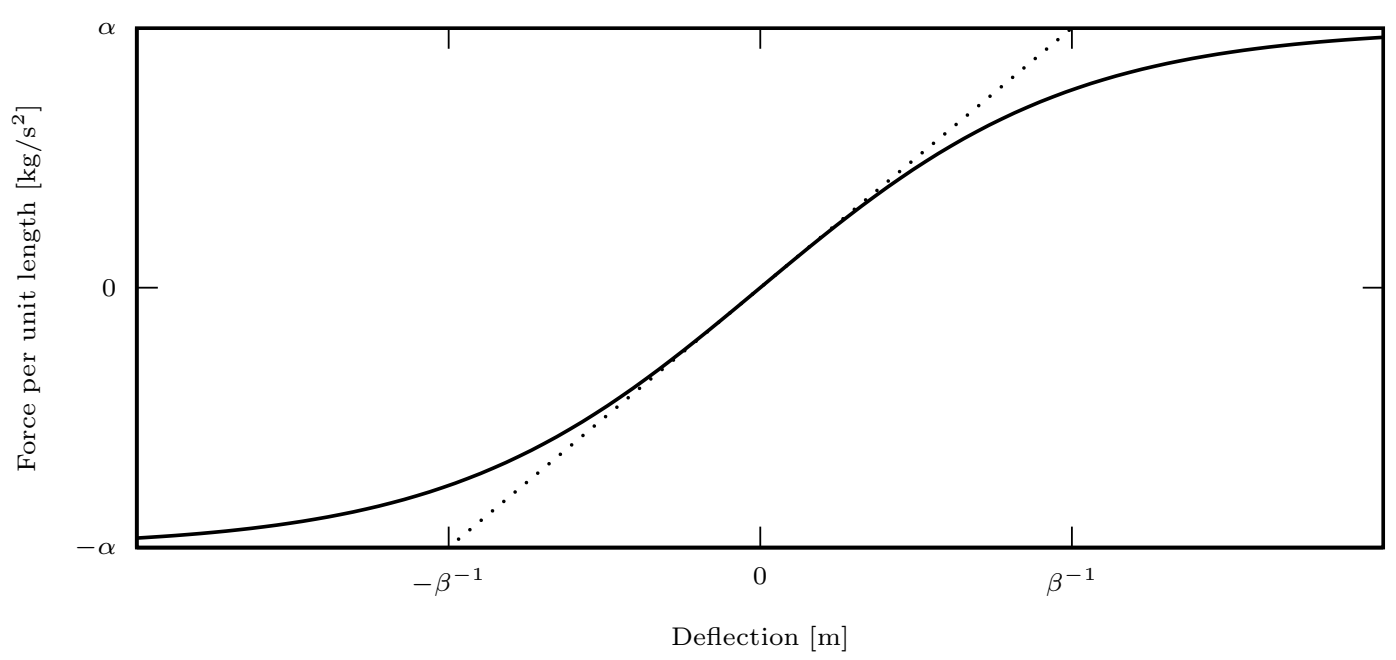

Figure 2.9: The relationship between the outer hair cell driven force and the hair bundle deflection can be represented by a sigmoid function (solid line). Due to saturation effects the force is limited to a value $\alpha(x)$. At the origin the sigmoidal function can be approximated by a linear function (dotted line) with a slope of $\alpha(x) \beta(x)$.

length changes, can be represented as

$$
\vartheta(x, \zeta(x, t))=\alpha(x) \operatorname{sig}(\beta(x) \zeta(x, t))
$$

where sig is a sigmoid function 1 as illustrated in figure 2.9 and $\alpha(x)$ and $\beta(x)$ are parameters that depend on the specific internal processes involved in the outer hair cell motility. The reason for describing the outer hair cell driven force by a sigmoidal relationship can be mainly found in saturation effects within the outer hair cells that limit the force. For small deflections of the hair-bundles these saturation-effects can be neglected and equation 2.34 can be linearly approximated by

$$
\vartheta(x, \zeta(x, t)) \approx \alpha(x) \beta(x) \zeta(x, t)
$$

\section{(cf. [1, 38, 28, 33])}

In order to calculate the force-feedback the deflection of the hair-bundles have to be determined on the basis of the vertical displacement of the basilar membrane. It is assumed that the motions of the tectorial membrane relative to the reticular lamina can be represented as a separate oscillator and that this subsystem is mainly stimulated by the vertical acceleration of the basilar membrane displacements. In terms of a linear differential equation and according to [28, 33] the deflection $\zeta$ of the hair bundles can therefore be written as

$$
m_{\mathrm{tm}}(x) \frac{\partial^{2} \zeta(x, t)}{\partial t^{2}}+h_{\mathrm{tm}}(x) \frac{\partial \zeta(x, t)}{\partial t}+k_{\mathrm{tm}}(x) \zeta(x, t)=g_{\mathrm{tm}}(x) m_{\mathrm{tm}}(x) \frac{\partial^{2} \xi(x, t)}{\partial t^{2}}
$$

\footnotetext{
${ }^{1}$ The sigmoid function used in this work is defined as $\operatorname{sig}(x):=1-\frac{2}{\mathrm{e}^{2 x}+1}$. The sigmoid function is chosen in such a way that its values ranges from -1 to 1 and that its slope at the origin is equal to 1.
} 


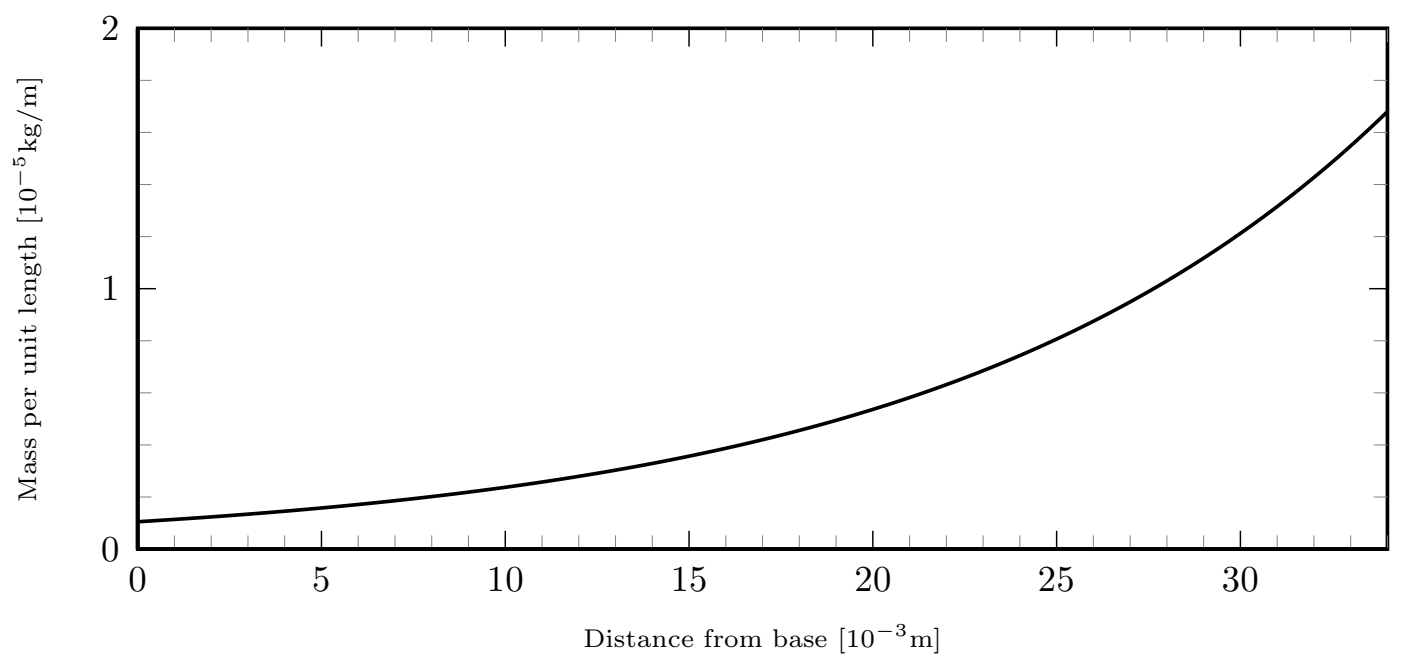

Figure 2.10: The mass (per unit length) of the tectorial membrane that is believed to be relevant for the inertial effects of the subordinate oscillator that describes the motion of the tectorial membrane relative to the reticular lamina.

where $m_{\mathrm{tm}}(x), h_{\mathrm{tm}}(x)$ and $k_{\mathrm{tm}}(x)$ are coefficient functions used for constituting the specific inertia, damping and stiffness of the oscillating subsystem. Due to the different orientation of the subsystem and due to some internal losses of the coupling, the external force acting on the subsystem is only a fraction of the vertical acceleration driven forces. This effect was taken into account by the additional gain function $g_{\mathrm{tm}}(x)$. According to Nobili et al. [33], it is assumed that the gain function is constant along the cochlear partition and that the effective force acting on the subsystem is only a tenth of the vertical force.

The effective mass $m_{\mathrm{tm}}(x)$ can be associated with the mass of the tectorial membrane. The radial width and transversal height of the tectorial membrane at the base of the cochlea can be assumed to be approximately $70 \mu \mathrm{m}$ and $15 \mu \mathrm{m}$ respectively. If both proportions are assumed to increase exponentially up to four times and if the density of the tectorial membrane is nearly equal to that of water, the mass of the tectorial membrane per unit length can be estimated as shown in figure 2.10. (cf. [31])

The damping is mainly caused by the shearing motion of the endolymph between the tectorial membrane and the reticular lamina. Due to the laminar regime of this shear, the damping coefficient can be estimated by

$$
\eta_{\text {end }} \frac{W_{\text {gap }}(x)}{H_{\text {gap }}(x)} \frac{\partial \zeta(x)}{\partial t}
$$

with $\eta_{\text {end }}, W_{\text {gap }}(x)$ and $H_{\text {gap }}(x)$ being the viscosity of the endolymph, the width and the height of the gap between the reticular lamina and the tectorial membrane at the position $x$. Under the assumption that the endolymph viscosity is approximately $1.5 \times 10^{-3} \mathrm{~kg} / \mathrm{m} \mathrm{s}$ (cf. [50]) and that the ratio between the radial width and the transversal height of the cleft 


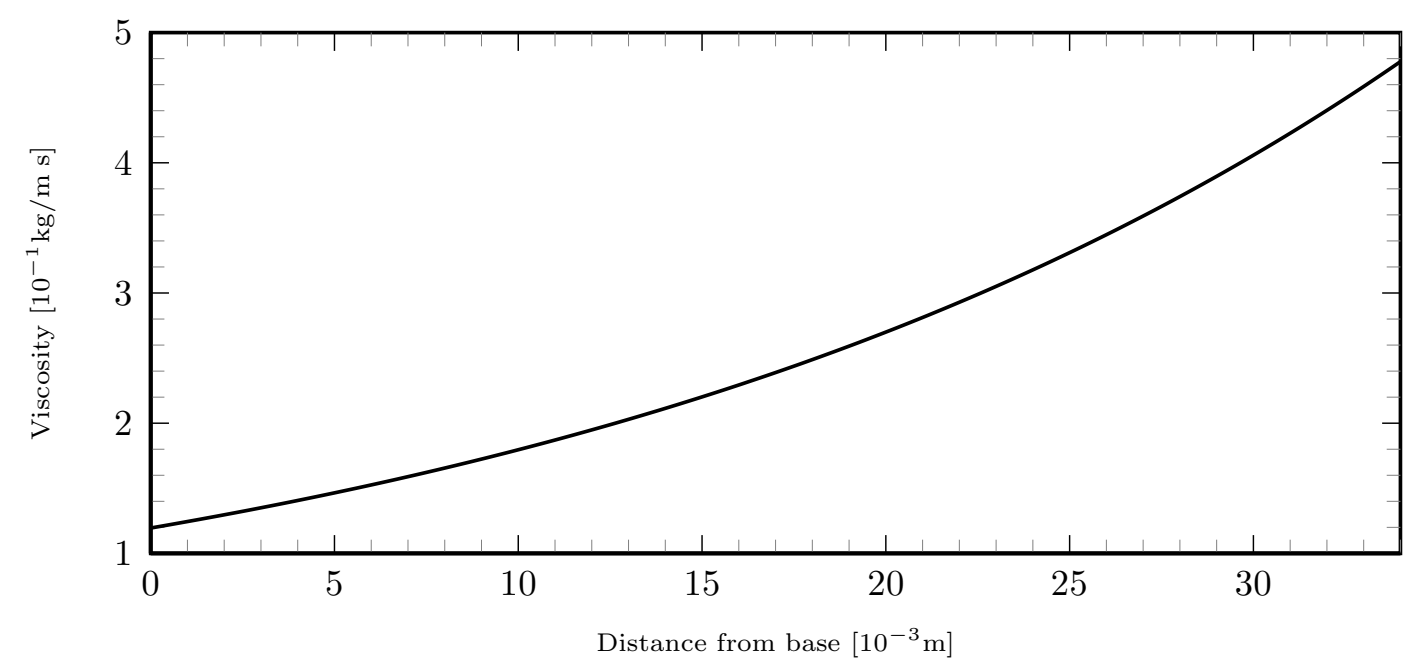

Figure 2.11: The viscosity of the secondary oscillator that causes the damping. It is assumed that the viscosity is mainly dominated by the shearing motions of the endolymph between the tectorial membrane and the reticular lamina.

increases from 80 at the base to 320 at the apex, the damping is specified as illustrated in figure 2.11.

It can be observed that the maximal displacement of the traveling wave in the active cochlea is shifted a little bit in the direction of the apex in contrast to the passive cochlea (cf. [38]). Having regard to this characteristic, the resonance frequency $f_{\text {res }}$ of the supplementary oscillator is believed to decrease exponentially from $18000 \mathrm{~Hz}$ at the base to $60 \mathrm{~Hz}$ at the apex. As a consequence, the stiffness can be uniquely determined by the relationship

$$
k_{\mathrm{tm}}(x)=m_{\mathrm{tm}}(x) \cdot\left(f_{\mathrm{res}}(x)\right)^{2} .
$$

As pointed out by Nobili et al. [33], at a specific location $x$ the inertial force will be nearly canceled by the stiffness driven force if the frequency of an acoustically-induced vibration is close enough to the local resonance frequency. In such a case, the oscillation (at this specific local region) is mainly dominated by the damping term and equation 2.36 can be represented as

$$
\frac{\partial \zeta(x, t)}{\partial t} \approx \frac{g_{\mathrm{tm}}(x) m_{\mathrm{tm}}(x)}{h_{\mathrm{tm}}(x)} \frac{\partial^{2} \xi(x, t)}{\partial t^{2}} .
$$

By integrating equation 2.39 over time and by using equation 2.35, the force that will be approximately applied to regions of resonant behavior can be written as

$$
\vartheta(x, \xi(x, t)) \approx \frac{\alpha(x) \beta(x) g_{\mathrm{tm}}(x) m_{\mathrm{tm}}(x)}{h_{\mathrm{tm}}(x)} \frac{\partial \xi(x, t)}{\partial t} .
$$

As a component of the right hand side of equation 2.30, the outer hair cell driven force $\vartheta$ acts directly on the motions of the basilar membrane. The configuration of equation 


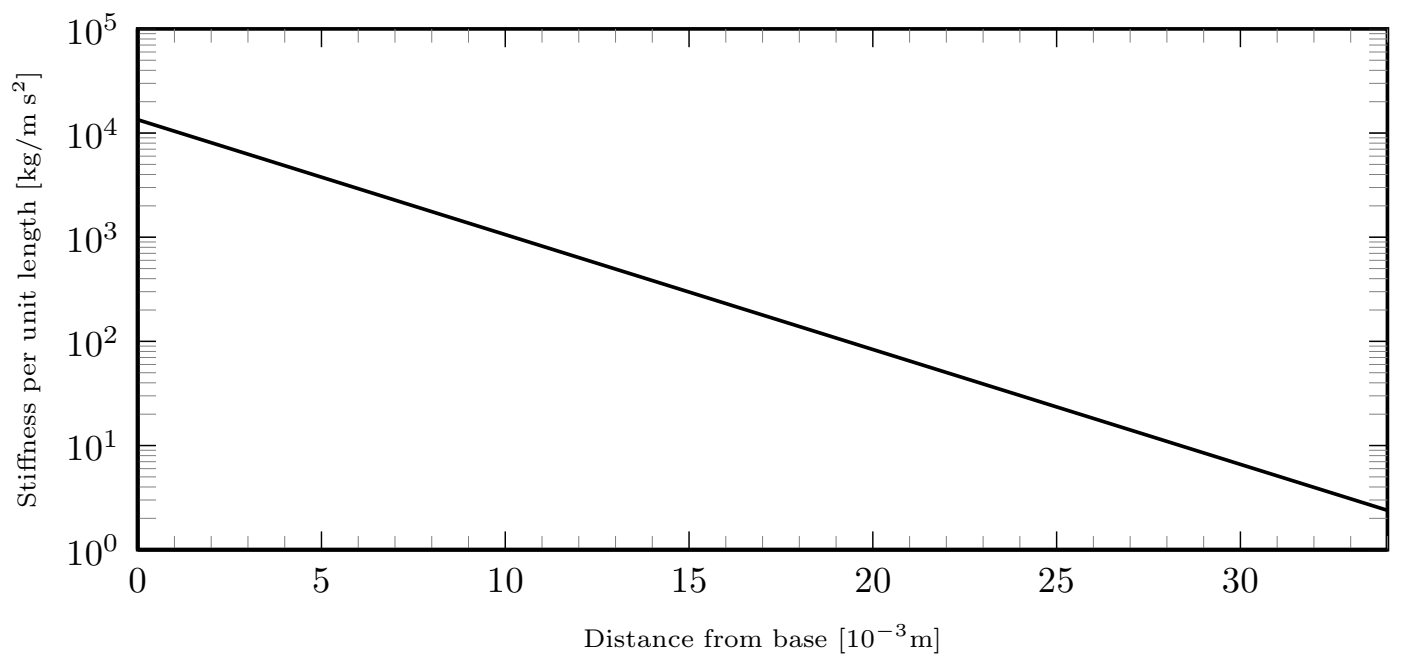

Figure 2.12: The effective stiffness of the subordinate oscillator induces a counteracting force that is applied in the opposite direction of its displacement.

2.40 indicates that the force works in principle as a negative damping term at regions of a nearly resonant behavior. In order to get numerical estimates of the parameters $\alpha(x)$ and

$\beta(x)$ that describe the sigmoidal shape of the functional relationship 2.34, it is assumed that the outer hair cell driven force can potentially neutralize the positional viscosity of the basilar membrane up to a certain degree. Therefore, if the positional damping term as given in equation 2.31 is equated with the damping term of equation 2.40, the coefficient function of equation 2.35 can be calculated to

$$
\alpha(x) \beta(x)=\frac{\lambda(x) \eta_{\mathrm{rad}} H_{\mathrm{ohc}}(x) h_{\mathrm{tm}}(x)}{W_{\mathrm{ohc}}(x) g_{\mathrm{tm}}(x) m_{\mathrm{tm}}(x)} .
$$

Thereby, the additional parameter function $\lambda(x)$ serves as a control parameter in order to specify the degree of damping cancellation. If $\lambda$ is equal to one, the total positional viscosity can potentially be canceled by the outer hair cell driven force. A value above one can also cancel damping effects caused by longitudinal shearing resistances. (cf. [28, 33])

\subsubsection{Equilibrium state}

If the first order system is stimulated by an harmonic vibration of the oval window, the system will reach a stable state of equilibrium after a certain amount of time has elapsed. According to Köster [24], the equilibrium state of the fluid can be expressed by harmonic oscillations of the individual field variables at fixed positions. In terms of sin- and cos- 


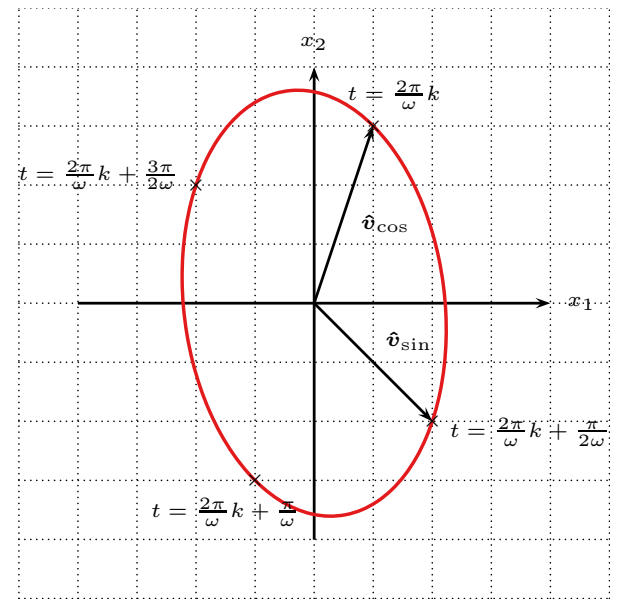

(a)

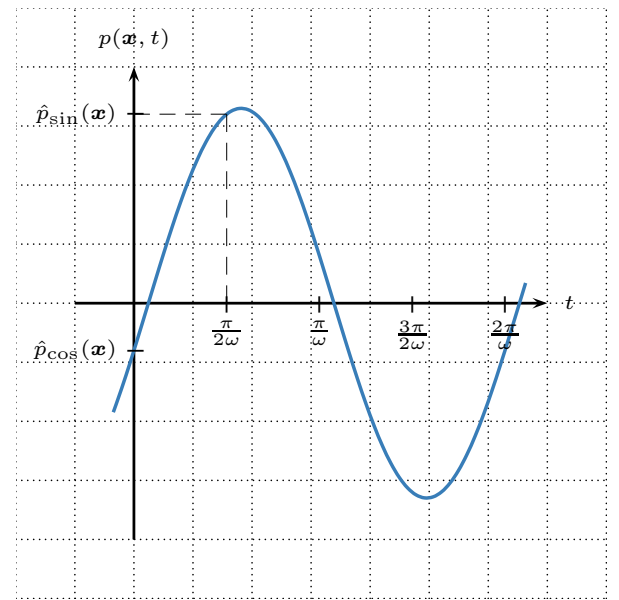

(b)

Figure 2.13: At any fixed location $\boldsymbol{x}$ within the fluid domain of the cochlea model, the equilibrium state can be clearly described by the sine-cosine decomposition. Figure (a) illustrates the equilibrium state of the velocity on the basis of the vectors $\boldsymbol{v}^{(\cos )}$ and $\boldsymbol{v}^{(\mathrm{sin})}$, whereas the pressure is characterized by the values $p^{(\cos )}$ and $p^{(\sin )}$ as it can be seen in figure $(b)$.

functions, the stable state can be written as

$$
\begin{aligned}
& \boldsymbol{v}^{(1)}(\boldsymbol{x}, t)=\boldsymbol{v}^{(\sin )}(\boldsymbol{x}) \sin (\omega t)+\boldsymbol{v}^{(\cos )}(\boldsymbol{x}) \cos (\omega t), \\
& p^{(1)}(\boldsymbol{x}, t)=p^{(\sin )}(\boldsymbol{x}) \sin (\omega t)+p^{(\cos )}(\boldsymbol{x}) \cos (\omega t) .
\end{aligned}
$$

Therefore, the oscillatory motion of the fluid can be uniquely characterized by the timeindependent functions $\boldsymbol{v}^{(\sin )}(\boldsymbol{x}), \boldsymbol{v}^{(\cos )}(\boldsymbol{x}), p^{(\sin )}(\boldsymbol{x})$ and $p^{(\cos )}(\boldsymbol{x})$. In this work, the representation of the equilibrium state with the help of equations 2.42 and 2.43 will be referred to as sine-cosine decomposition. Sometimes it is more convenient to use the complex exponential form in order to represent the equilibrium state. Then, the velocity- and pressure-field are given by

$$
\begin{aligned}
& v_{i}^{(1)}(\boldsymbol{x}, t)=v_{i}^{(\mathrm{amp})}(\boldsymbol{x}) \mathrm{e}^{\left[\omega t+\varphi_{v_{i}}(\boldsymbol{x})\right] \mathrm{i}}, \\
& p^{(1)}(\boldsymbol{x}, t)=p_{i}^{(\mathrm{amp})}(\boldsymbol{x}) \mathrm{e}^{\left[\omega t+\varphi_{p_{i}}(\boldsymbol{x})\right] \mathrm{i}}
\end{aligned}
$$

where the superscript (amp) denotes the amplitude and $\varphi$ the argument of the associated quantity. Such a complex representation must, of course, be understood in the sense that only its real part specifies the value of the physical quantity. 


\subsection{The Second Order System}

\subsubsection{The Acoustic Streaming Subproblem}

The acoustic streaming subproblem is established on the basis of the second order system that results from the perturbation approach as described in chapter 2.2.4. In steady state, the second order equations are associated with the second harmonic fields in combination with a steady flow field. While the determination of the second harmonic field is of no interest to this work, the evaluation of the steady flow field yields a first order approximation of acoustic streaming. In order to extract the steady flow field (hereinafter indicated by the superscript $(d c)$ ) from the second order system, the operator $\langle\cdot\rangle$ that determines the temporal average of time-dependent functions can be applied. The mean of the second order equation that is associated with the balance-of-mass principle 2.1 can be written as

$$
\rho^{(0)} \operatorname{div} \boldsymbol{v}^{(d c)}=\tilde{m}
$$

where the term $\tilde{m}$ corresponds with a virtual mass-source. This source distribution is caused by the first order acoustic subproblem and describes the supply of mass (cf. [9]). In terms of the first order fields, the mass-source can be evaluated to

$$
\tilde{m}=-\frac{1}{c_{0}^{2}} \operatorname{div}\left\langle p^{(1)} \boldsymbol{v}^{(1)}\right\rangle
$$

Similarly, the steady components of the secondary flow fields must fulfill the time-averaged second order version of the balance-of-momentum principle 2.2. which is given by

$$
\operatorname{grad} p^{(d c)}-\left(\zeta+\frac{\eta}{3}\right) \operatorname{grad} \operatorname{div} \boldsymbol{v}^{(d c)}-\eta \Delta \boldsymbol{v}^{(d c)}=\tilde{\boldsymbol{f}}
$$

where $\tilde{\boldsymbol{f}}$ denotes a virtual force-source distribution. The force-source acts as an external volume-force and depends on the acoustic field. By using first order terms, the force-source distribution can be expressed as

$$
\tilde{\boldsymbol{f}}=-\frac{1}{c_{0}^{2}}\left\langle p^{(1)} \frac{\partial \boldsymbol{v}^{(1)}}{\partial t}\right\rangle-\rho^{(0)}\left\langle\left(\operatorname{grad} \boldsymbol{v}^{(1)}\right) \boldsymbol{v}^{(1)}\right\rangle .
$$

According to Bradley [9], the correct treatment of the boundary condition leads to an important boundary driven mechanism of acoustic streaming which was often neglected by other works. This mechanism is based on the rigorous distinction between the Eulerian and the Lagrangian specification of motion as described at the beginning of chapter 2.2. As can be seen from equations 2.11 and 2.12, the difference between both kinds of descriptions at the boundary arises from considering the full Taylor expansion. While both specifications are identical in the case of the first order perturbation approximation, the difference becomes apparent by taking the second order terms of the perturbation expansion into account. 
By substituting the respective perturbation expansion for the field variables of equation $[2.12$ and by applying the mean-operator, the time-averaged second order boundary condition can be written as

$$
\boldsymbol{v}^{(d c)}=-\left\langle\left(\operatorname{grad} \boldsymbol{v}^{(1)}\right) \boldsymbol{\zeta}^{(1)}\right\rangle
$$

The mean of the second order Lagrangian velocity component vanishes, since each point of the boundary oscillates around a fixed locations. The displacement $\boldsymbol{\zeta}$ can be evaluated by solving the initial value problem which is given by equation 2.11. By performing the usual perturbation approach, the first order approximation of the displacement can be calculated to

$$
\zeta_{i}^{(1)}\left(\boldsymbol{x}_{0}, t\right) \approx \int v_{i}^{(1)}\left(\boldsymbol{x}_{0}, t\right) \mathrm{d} t=\frac{v_{i}^{(1)}}{\mathrm{i} \omega} .
$$

By using equations 2.50 and 2.51 in combination with vector identities2 2 , the mean of the second order Eulerian velocity can be expressed as

$$
\boldsymbol{v}^{(d c)}=-\frac{1}{\rho^{(0)} c_{0}^{2}}\left\langle p^{(1)} \boldsymbol{v}^{(1)}\right\rangle+\frac{1}{2} \operatorname{rot}\left\langle\boldsymbol{\zeta}^{(1)} \times \boldsymbol{v}^{(1)}\right\rangle .
$$

As discussed by Bradley [9], this equation allows a better understanding of the underlying mechanisms of the resultant mean flows than equation 2.50. Under the assumptions of pure rectilinear (not elliptical) motions of the moving boundaries, it becomes apparent that the first term of the right hand side of equation 2.52 acts as a sink (or a source) since the resultant mean flow has the same direction as the first order velocity (cf. [9, 26]). The second term was often neglected in other works. The magnitude of the cross product of the velocity and the displacement is proportional to the enclosed area of the ellipse that is formed by the trajectory of the associated fluid particle. The direction of the resultant vector is perpendicular to this surface. Due to the rectilinear motion of the boundaries, this cross product vanishes at the boundaries. But if the fluid motion of the adjacent fluid layers is characterized by elliptical trajectories, the curl of this cross product will induce a slipping mean flow at the boundaries. A detailed consideration of the physical origin of these mean flows can be found in [9].

\footnotetext{
${ }^{2} \operatorname{rot}(\boldsymbol{a} \times \boldsymbol{b})=(\operatorname{grad} \boldsymbol{a}) \boldsymbol{b}-(\operatorname{grad} \boldsymbol{b}) \boldsymbol{a}+\boldsymbol{a} \operatorname{div} \boldsymbol{b}-\boldsymbol{b} \operatorname{div} \boldsymbol{a}$
} 


\section{Chapter 3}

\section{Implementation}

This chapter is concerned with the numerical implementation of the mathematical model of the cochlea system as described in chapter 2 The mathematical model consists of a number of different partial differential equations in combination with specific boundary conditions, which have to be fulfilled on their associated domain. In this work the finite element method is used in order to calculate a numerical solution of this system of equations. The finite element method is a powerful technique for the numerical evaluation of (initial) boundary value and eigenvalue problems for a wide range of applications. Within the framework of the finite element analysis, the solution of a given differential equation is approximated by a linear combination of a set of specific basis functions. In contrast to some other methods (e.g. Galerkin methods), these basis functions (which are also referred to as form functions or interpolation functions) are characterized by its systematic construction on a set of individual simple sub-domains that form the entire domain of interest. (cf. [36])

The schematic design of the global simulation process for the computation of the acoustic streaming field within the inner ear can be seen in algorithm 3.1. In principle only the stimulation frequency of the harmonic stapes displacement within the oval window and the outer hair cell activity parameter, which controls the influence of the additional outer hair cell force, are required in order to start the numerical computation. All other physical parameters and properties are predefined (cf. chapter 2) and can be configured within an external parameter file.

In a first step (cf. line 2 of algorithm 3.1), the fluid domain as well as the onedimensional domain that hosts the basilar membrane have to be divided into a set of subdomains, the so-called elements. The choice of these elements and the underlying strategy of the discretization process (also known as meshing) are discussed in chapter 3.1. On the basis of these meshes, finite element spaces can be systematically introduced by defining appropriate approximation functions upon the individual subdomains (cf. line 3). Section 3.3 deals with the specific configuration of the different function spaces which are used for the approximation of the different field functions. In order to establish a discretized formulation of the mathematical model, the differential equations have to be 
initially transformed into a so-called weak form (also known as variational formulation). In contrast to the original differential equation, the requirements on the solution of the weak formulation with respect to its differentiability are weakened. Furthermore, the solution of the weak form does not need to fulfill the differential equation at individual specific points, but rather (to some extent) on average over an arbitrarily chosen region in the sense of a specific weighted integral statement. (cf. [36, 23]) The derivations of the individual variational formulations are presented in section 3.2. On the basis of the weak formulations a spatial discretization can now be performed by using appropriate linear combinations of the basis function for the spatial functions. With respect to the first order acoustic subproblem, this leads to a number of different linear systems of ordinary differential equations (cf. chapter 3.4.1). Its associated matrices can be stepwise assembled by performing local calculations for each element (cf. line 4). In the next step, which is associated with line 5 of algorithm 3.1, the individual systems are combined to a single system of ordinary differential equations. The resultant matrix equation can be seen in chapter 3.4.2. Lines 7 to 12 are associated with the temporal discretization of the ordinary differential system of equations. The time integration scheme that is used in this work is presented in chapter 3.5. The fully discretization process of the first order acoustic subproblem finally results in a single linear system of equations per time-step.

If the stable state of equilibrium is reached at which all field variables can be described by an harmonic function (cf. chapter 2.3.4), the acoustically driven motions can be evaluated on the basis of the results of the first order subproblem. Similar to the acoustic subproblem, individual submatrices can be constituted by using the weak formulations of the second order subproblem. Although, no interactions between the fluid and its adjacent structures are considered by the second order flows, an overall matrix, which contains all submatrices, has to be assembled because a mixed formulation is used for the description of the fluid motions. The load vector depends on the acoustic field and can be determined by calculating certain mean values over one cycle. As discussed in chapter 3.4 .3 , the steady state motion of the acoustic streaming problem can be represented by a single linear system of equation which can be solved in one step.

The resultant linear systems of equations have to be solved by the use of an appropriate solver. As discussed in chapter [3.6, the generalized minimal residual method is applicable for approximating the solutions of the first as well as the mean second order system. Furthermore, the rate of convergence can be significantly improved by using an incomplete $L U$ decomposition as a preconditioner (cf. section 3.6.3).

At the end of the algorithm, the results are analyzed, prepared, recorded and visualized by the routines of the post-processing.

The computational realization is based on the finite element toolbox AlBERTA [43] and the PETSC library [2]. The toolbox AlBeRTA provides basic data structures and routines in order to support the establishment of appropriate meshes and finite element spaces. In addition, it maintains great flexibility to allow the direct integration and implementation of new numerical methods. (cf. [42, 44]) After finishing the respective finite element discretizations, the resultant systems of equations are solved with the help of the PETSC 
library [2].

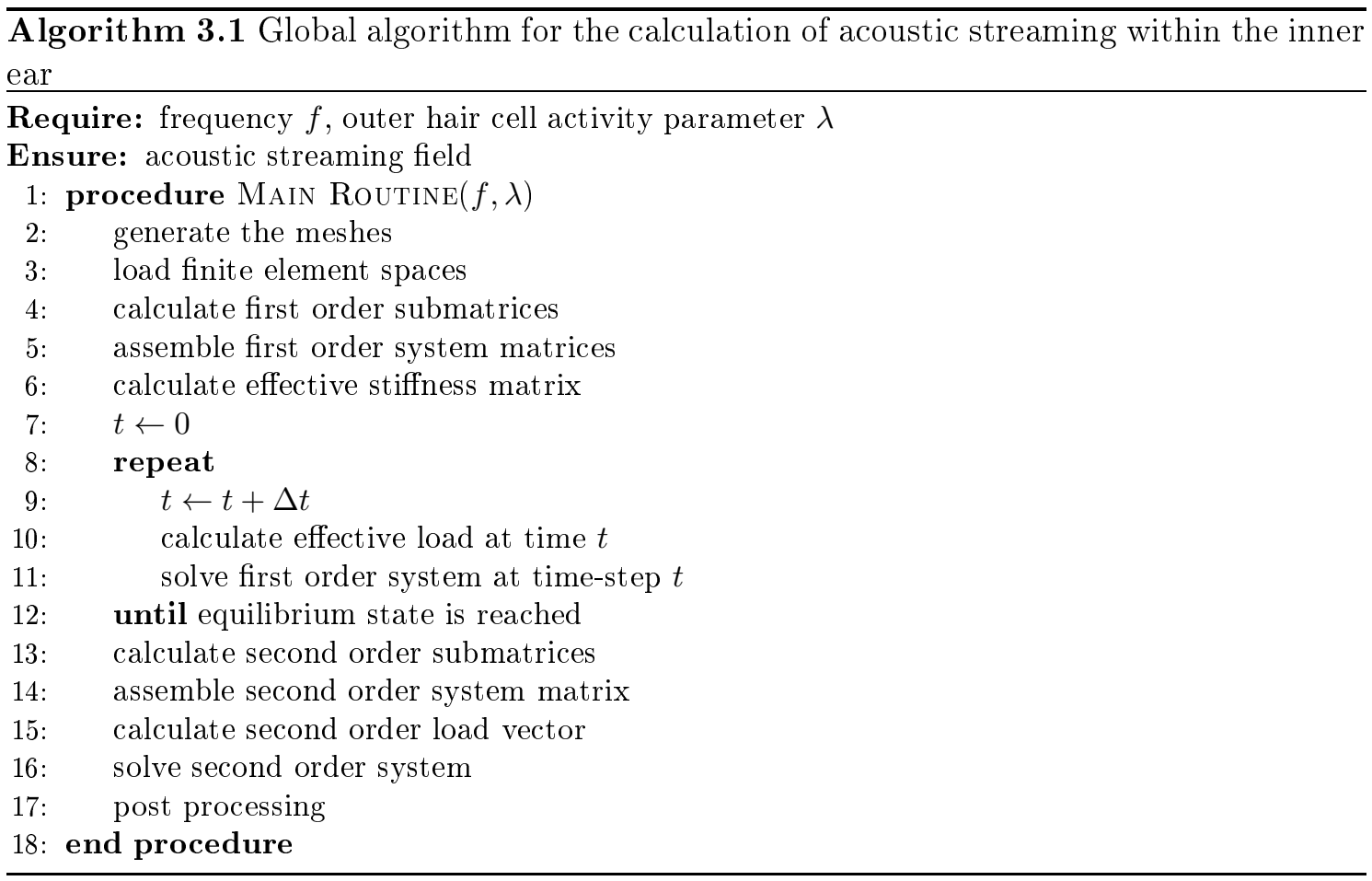

\subsection{Mesh Generation}

According to line 2 of algorithm 3.1, the first step of the numerical simulation process presented here is an adequate construction of the underlying mesh which can be used for the finite element calculations.

The starting point of the mesh generation is a coarse triangulation of the upper duct of the cochlear system which is initially represented by a rectangular box as it is, in principle, illustrated in figure 3.1(a). In order to achieve the final mesh, this triangulation is modified and extended by a three-stage process. The first step consists of a refinement of the mesh in order to minimize potential errors between the exact solution and its numerical approximation. In contrast to a global refinement of the whole mesh, the computational effort of the simulation process can be significantly reduced by using only local refinements. The concrete refinement strategy used in this work is discussed in chapter 3.1.2. At the second stage the geometry of the scala tympani and scala vestibuli above and below the cochlear partition has to be taken into account by the meshing routines (cf. chapter 3.1.3). Finally, an additional one-dimensional mesh is introduced which is needed in connection with the numerical implementation of the mechanical properties of the basilar membrane and its outer hair cell feedback force. The specific construction of this mesh with respect to the individual fluid triangles above and below this partition is reviewed in chapter 3.1.4. 


\subsubsection{Reference Elements}

According to [42] it is useful to introduce two different reference elements in order to simplify the numerical evaluations with respect to the finite element analysis upon each individual element.

A set $X$ of $d+1$ different points $\boldsymbol{x}_{i} \in \mathbb{R}^{n}$ will constitute a single element (also referred to as simplex) of the mesh $M$ if the vectors $\boldsymbol{x}_{1}-\boldsymbol{x}_{0}, \ldots, \boldsymbol{x}_{d}-\boldsymbol{x}_{0}$ are linear independent. The element $S^{d}$ is defined as the convex hull of these points, or in terms of a mathematical formulation $S^{d}$ can be written as

$$
S^{d}(X)=\left\{\sum_{i=0}^{d} \beta_{i} \boldsymbol{x}_{i} \mid \beta_{i} \geq 0, \sum_{i=0}^{d} \beta_{i}=1, \boldsymbol{x}_{i} \in X\right\}
$$

From a geometrical point of view a single simplex can be associated with a triangle in the case of $d=2$ or, respectively, by a line-segment in the case of $d=1$.

For reasons of simplicity, the numerical quadrature (which will be needed in the context of the finite element analysis) is not performed on each individual element itself but rather on a normalized element. In this work, this normalized element is given by

$$
S_{(\text {nor })}^{d}:=S^{d}\left(\mathbf{0}, e_{1}, \ldots, e_{d}\right)
$$

where $\boldsymbol{e}_{i}$ are the unit vectors of the coordinate system. As it can be easily seen, the element $S_{(\text {nor })}^{d}$ can be linked to a specific element $S^{d}(X)$ by a linear affine mapping $\boldsymbol{x}(\boldsymbol{\nu})$ (cf. [42]).

In contrast to this normalized element, it is more comfortable to establish the basis functions of the finite element spaces by the use of barycentric coordinates due to its symmetry properties. On the basis of the element

$$
S_{(\mathrm{bar})}^{d}:=S^{d}\left(e_{1}, \ldots, e_{d+1}\right)
$$

the barycentric coordinates are associated with the coefficient $\beta_{i}$ which are introduced by the definition 3.1. Therefore, the element $S_{(\mathrm{bar})}^{d}$ will be referred to as barycentric simplex. The relation between the barycentric coordinates on the one hand and the world coordinates of a specific or the normalized element on the other hand can be described by an invertible mapping.

As illustrated by the following scheme, each element $S^{d}(X)$ of a mesh can therefore be parameterized over the normalized element $S_{(\text {nor) }}^{d}$ and the barycentric representation $S_{(\text {bar })}^{d}$ 
by the invertible mappings $\boldsymbol{x}(\boldsymbol{\nu})$ and $\boldsymbol{x}(\boldsymbol{\beta})$.

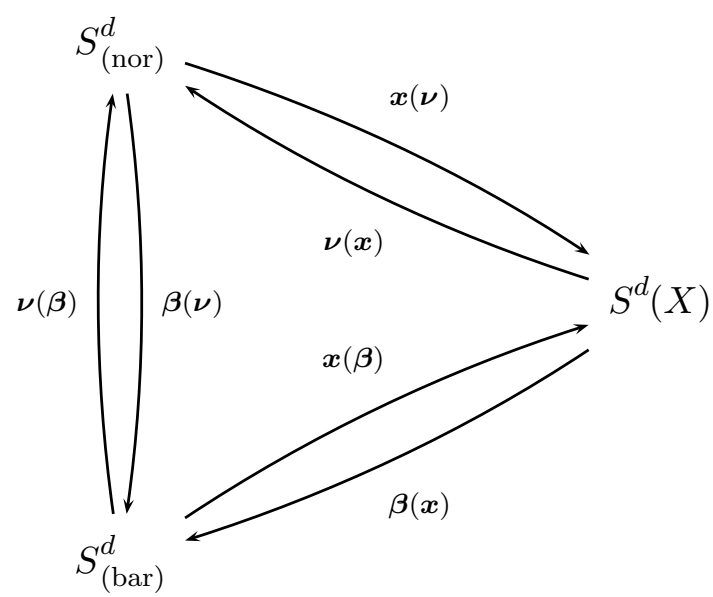

In addition, these both mappings induce uniquely an invertible mapping $\boldsymbol{\beta}(\nu)$ from the normalized coordinates to the barycentric coordinates.

\subsubsection{Refinement of the mesh}

With regard to the refinement of a triangular mesh, it must be kept in mind that the resultant mesh has also to fulfill the characteristics of a regular triangulation. Thereby, the term regular triangulation refers to a mesh in which the intersection of any two triangles is either the entire edge of both triangles, a single vertex or empty. Therefore, the splitting of only one triangular element into two sub-triangles will probably lead to a so-called hanging vertex at the bisected edge, if this edge does not belong to the outer boundary of the triangulation.

A relative simple method for mesh refinement would be a global refinement of all elements into e.g. $k^{2}$ uniform sub-elements in such a way that each edge would be divided into $k$ equidistant edges. But it is apparent that such a global refinement method leads to a significant increase of computational effort and memory consumption with respect to a finite element analysis. Therefore, in order to minimize the computing time and the memory requirements on the one hand and, simultaneously, to maximize the accuracy of the finite element approximation on the other hand, the objective of local refinement strategy is to construct a mesh as optimal as possible by taking both requests into account (cf. [42]).

Furthermore, particular attention should be paid to the quality of individual elements. A large distortion of a triangle is characterized by considerable differences of its individual side lengths and angles respectively. Since large distortions are usually accompanied by an higher approximation error, the refinement method should split the triangles in such a way that its sub-elements are as equilateral as possible. 


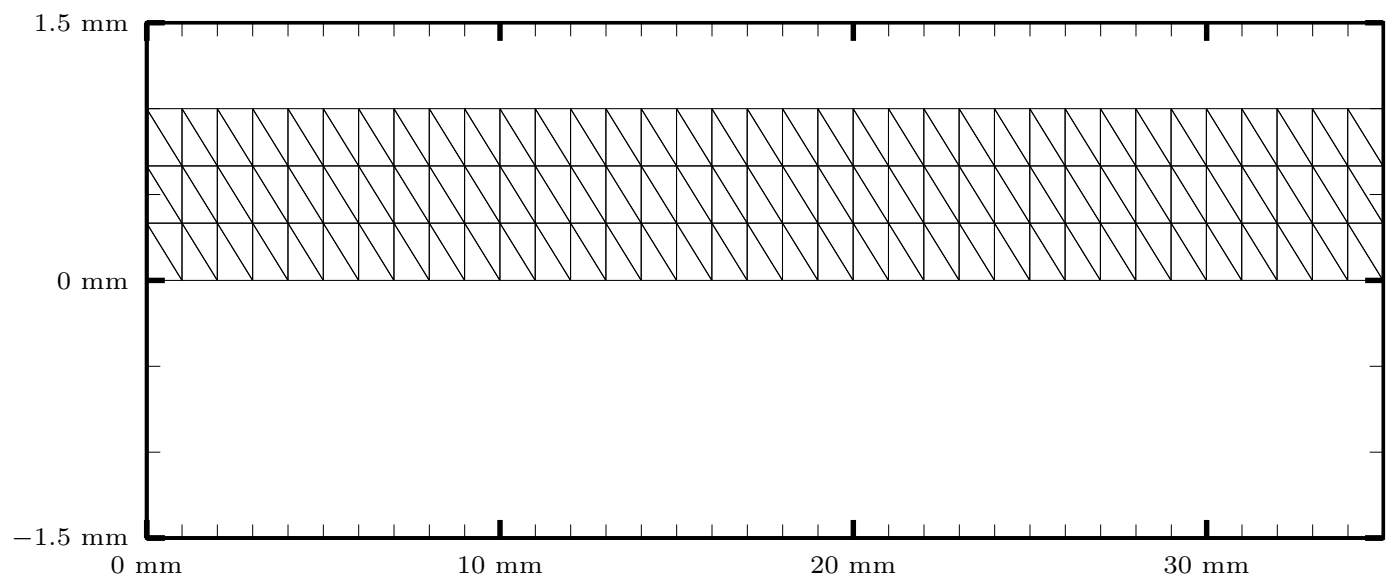

(a)

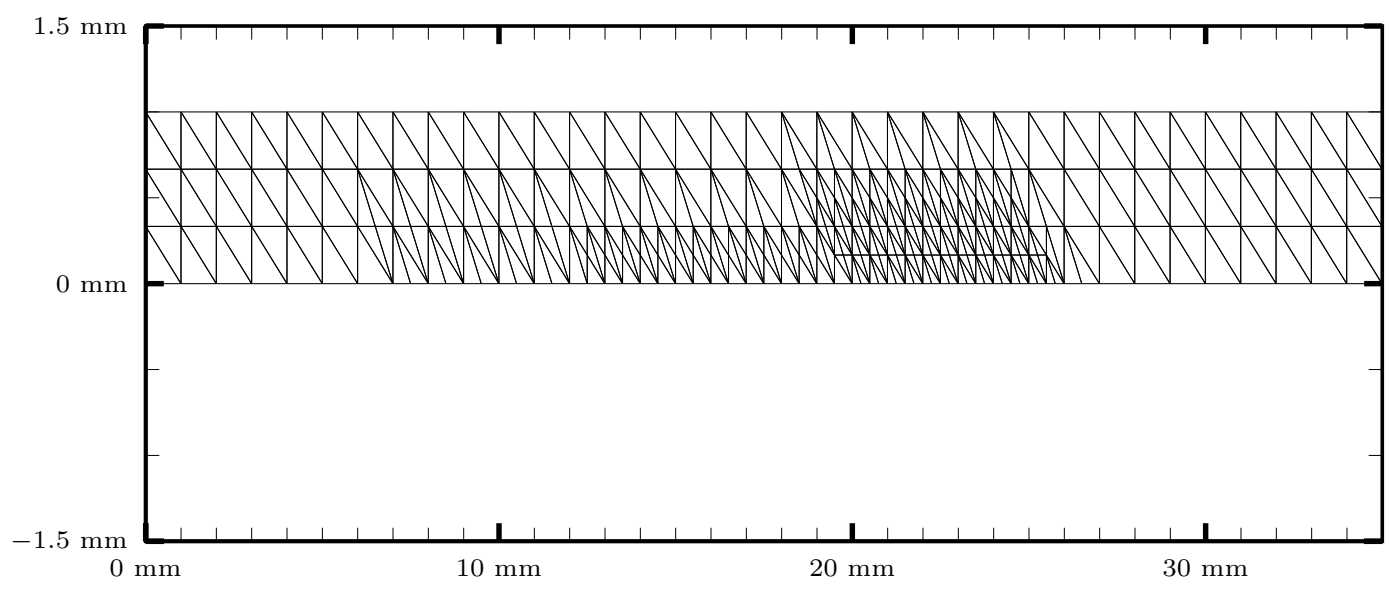

(b)

Figure 3.1: On the basis of a coarse mesh (a) that represents the scala vestibular a recursive routine locally refines the mesh in order to reduce the error of the finite element computations. The specific refinement strategy is based on an heuristic approach. The resulting mesh (b) is characterized by a gradual increase of its density along the basilar membrane until a point is reached where the traveling wave will probably be dissipated. 
The method used in this work for refining the coarse mesh is based on an initial markingroutine which specifies for each element the number of refinements that should be performed by the refinement procedure. The typical traveling wave motion of the basilar membrane displacement (as described in detail in chapter 4.1.3) is a result of its interactions with the adjacent fluid. Therefore, it can be expected that these displacement pattern will be reflected in the fluid motion to a certain extent. The significant increase of the amplitude of the traveling wave up to a maximum at the characteristic place in combination with a substantial decrease of its wave length indicates that the fluctuations of the field variables increase along the cochlear partition until the characteristic place is reached.

Another important aspect (as discussed in detail in chapter 4.1.4) is that the field variables within the thin Stokes boundary layer next to the basilar membrane should be accurately approximated by the finite element space. According to Lighthill [26], the thickness of this Stokes boundary layer can be estimated to range from about $10 \mu \mathrm{m}$ to $400 \mu \mathrm{m}$ in dependence of the stimulation frequency of audible sound.

On the basis of these heuristic arguments, the number of refinements of the elements next to the basilar membrane is gradually increased along the partition until a specific location is reached. This location is specified by the expected decay of the traveling wave, which can be estimated by using the frequency-position map from Greenwood [18] as described in chapter 4.1.3.

After the elements have been marked according to the strategy described above, the refinement algorithm can be performed. In doing this, the mesh is repeatedly traversed until no element of the mesh is marked anymore by a positive refinement number. At each element, which is labelled with a positive refinement number, a subroutine is called for the purpose of performing a local refinement. If necessary, not only the triangle itself, but also elements in the neighbourhood have to be refined in such way that the regularity of the mesh is preserved. The recursive subroutine used in this work is schematically described in algorithm 3.2 and already provided by the AlBERTA library [42].

In order to ensure the quality of the mesh, the splitting of a single triangle is only allowed at the midpoint of its longest edge. To avoid a hanging vertex, the algorithm will only perform a refinement if the longest edge of the triangle is also the longest edge of its adjacent triangle. In this case, both triangles can be refined simultaneously by using the midpoint of the common edge as a new vertex of all four sub-elements. Otherwise, the neighbouring triangle must first be refined until the common edge is at least as long as both other sides of the adjacent triangle. Therefore, the local refinement procedure is a recursive process and a single refinement of an individual triangle can entail a lot of other refinements of other triangles.

An example of the resulting mesh is illustrated in figure 3.13.1(b), It should be noted that the meshes that are generated for the finite element calculations are, of course, considerably finer than the meshes illustrated in this work. 


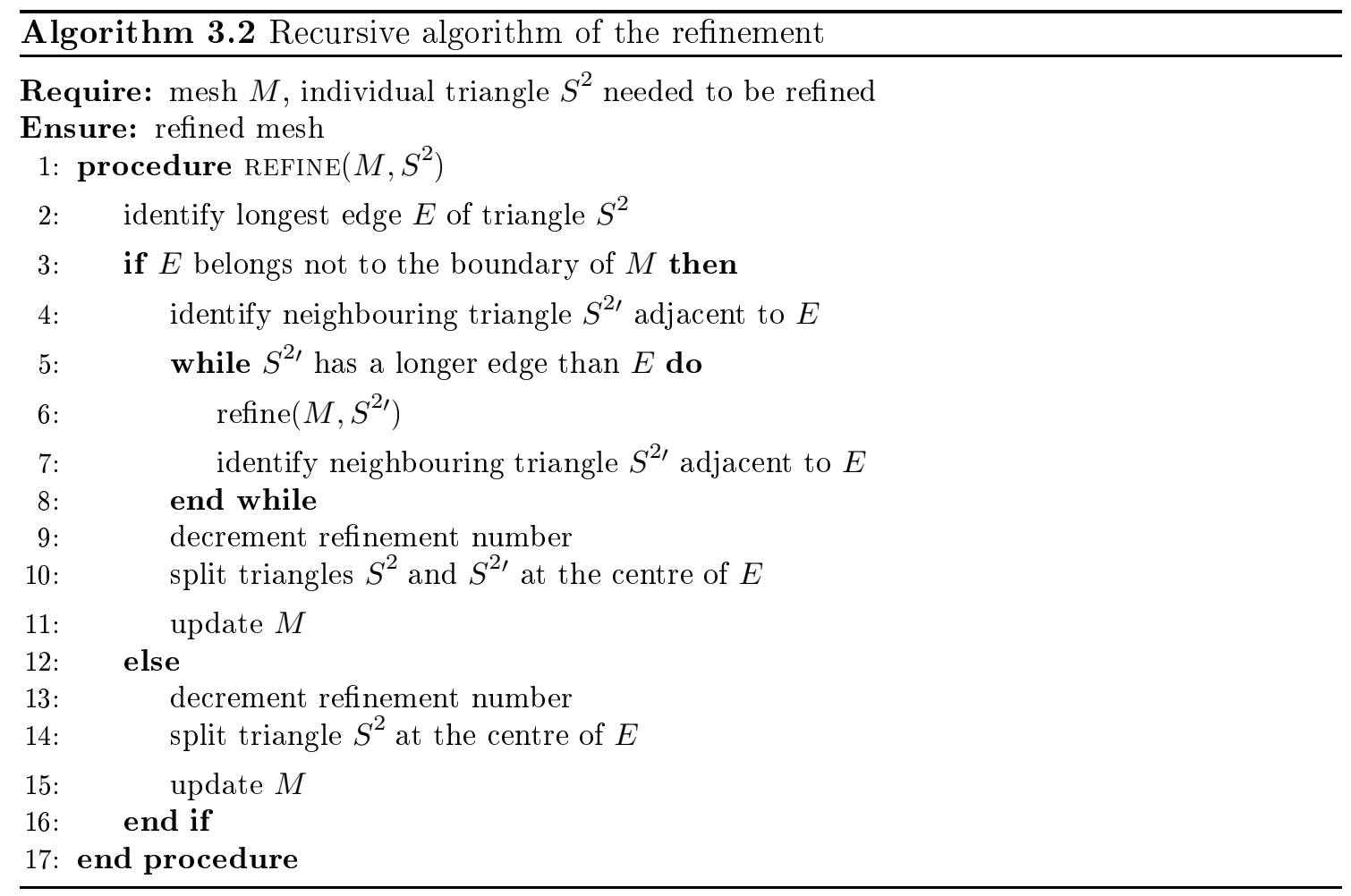

\subsubsection{Shape of the Mesh}

Up to now, only the upper half of the mesh, which represents the scala vestibular, is generated by the refinement procedure of a coarse rectangular triangulation. The mesh can now be supplemented by reflecting each element with respect to the longitudinal axis which contains the cochlear partition. The advantage of this approach is that the arrangement of edges that are adjacent to the cochlear partition and that belong to the upper duct is in conformity with the arrangement of the edges on the other side of the partition. Although there seems to be a connection of the mesh through the cochlear partition, the elements above and below the basilar membrane are separated from each other. Therefore, the line segments that are adjacent to the basilar membrane belong to the outer boundary of the mesh. From the technical point of view, the line segments at the cochlear partition must therefore be implemented in duplicate in order to represent the boundary between the basilar membrane and the scala vestibular as well as the boundary between the basilar membrane and the scala tympani. In order to connect both chambers at the helicotrema, the upper and the lower mesh are glued together at the apex. This means that the corresponding segments and vertices at the helicotrema are - in contrast to the cochlear partition - shared by both chambers. As shown below, this specific configuration facilitates the numerical implementation of the mutual interactions between the basilar membrane and the fluid at both sides. A positive side effect is that the reflection of the refined upper part reduces the computational effort, since the expensive refinement-routine 
has only to be performed for the half of the mesh. The supplemented mesh that results from the reflection as described above can be seen in figure 3.2](a),

Finally, the mesh has to be adapted in such a way that the shape of both chambers (as already discussed in chapter 2.1) is sufficiently represented by the fluid domain. Therefore, the transversal heights of the scala vestibular and scala tympani are modified according to the measurements from Wysocki [52] by multiplying the vertical coordinates with appropriate scaling factors. As a consequence, the boundary is linearly approximated by the triangular elements. In comparison to the model simplifications made above, the error that arises due to the linear approximation of the smooth boundaries may be considered as negligible. The effect of the vertical scaling is illustrated in figure 3.2)(b).

\subsubsection{Basilar Membrane as a Submesh}

The fluid domain is just one component of the whole cochlea model. In addition to the fluid domain, also the basilar membrane and its interactions with the fluid have to be taken into account by the numerical implementation. As already described above (cf. chapter 2.1), the basilar membrane should be represented by an one-dimensional line between the fluid filled chambers. The additional one-dimensional mesh is constructed in such a way that the individual line segments coincide with the corresponding boundary edges of the fluid domain. In summary, the cochlear partition consists of three different virtual "layers" of the same line-segments. The first two layers are associated with the fluid domain and act as the boundaries between the cochlear partition and the lower chamber or, respectively, the upper chamber. The third layer represents the basilar membrane for the implementation of its physical properties. The identical arrangement of each layer with respect to the location and width of each line segment is not mandatory, but it significantly simplifies the numerical realization of the fluid structure interaction. Therefore, the basilar membrane can in principle implemented as a submesh of the fluid domain, since its set of vertices is a subset of the vertices that belong to the fluid mesh.

\subsection{Variational Formulation}

The finite element method is based on the variational formulation of the boundary value problem. The derivation of the variational formulation can be divided into three steps:

1. In the first step, the partial differential equations of the boundary value problem must be multiplied with test-functions. In this context, the test-functions have to be chosen in such a way that they are in agreement with the respective boundaryconditions.

2. After that, the resultant statements must be integrated over the domain of interest.

3. If possible, the requirements concerning the differentiability of individual field variables should be finally weakened by applying Green's first identity. 


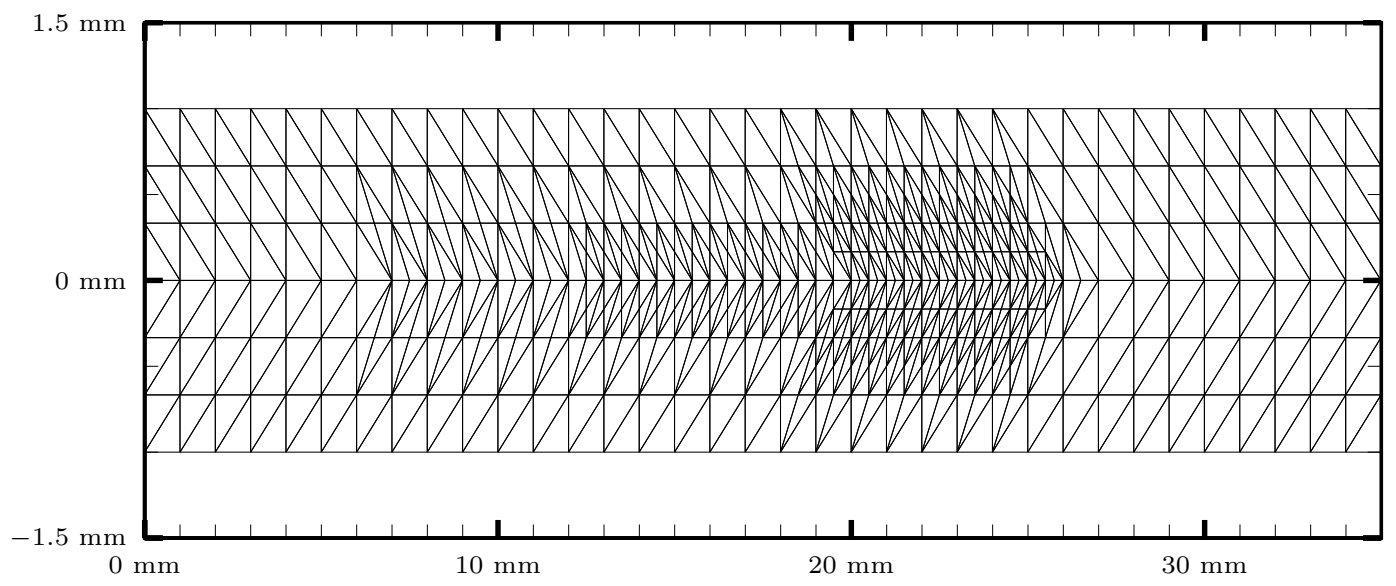

(a)

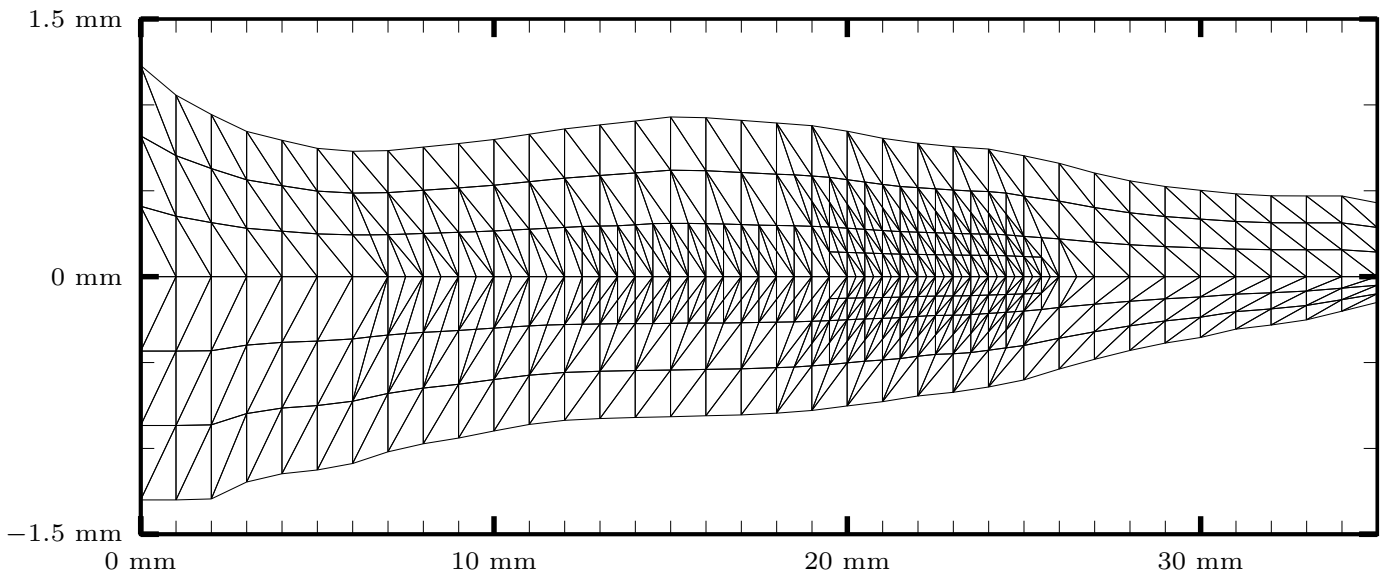

(b)

Figure 3.2: (a) The discretization of the fluid domain can be supplemented by reflecting the upper half of the mesh with respect to the longitudinal axis. In contrast to the cochlear partition, where both parts of the mesh are separated from each other, the mesh has to be connected at the helicotrema. (b) On the basis of the measurements from Wysocki [52], the shape of the cochlea can be linearly approximated by applying a scaling procedure with respect to the vertical axis. 
As can be seen from the structure of this section, the different weak forms can be associated with the individual components of the cochlear model as illustrated in figure 1.3

For reasons of simplicity this chapter uses the Einstein summation convention for establishing the weak forms. Therefore, all terms, where an index occurs twice, are to be understood as the summation of the term over all possible values intended for this index.

\subsubsection{The First Order System}

Acoustic Subproblem The fluid dynamics of the first order problem can be mainly described by the first order conservation equations of mass 2.22 and linear momentum 2.23. The weak form of the first order equation of the conservation of momentum is given by

$$
\rho^{(0)} \int_{\Omega} \bar{v}_{i} \frac{\partial v_{i}^{(1)}}{\partial t} \mathrm{~d} \boldsymbol{x}+\int_{\Omega} \frac{\partial \bar{v}_{i}}{\partial x_{j}} \sigma_{i j}^{(1)} \mathrm{d} \boldsymbol{x}-\int_{\Gamma} \bar{v}_{i} \sigma_{i j}^{(1)} n_{j} \mathrm{~d} x=0
$$

where the line above a variable indicates the test-functions. Due to the boundary condition 2.15, the boundary integral vanishes. According to the specification of the stress tensor 2.7, the second integral can be expanded to

$$
\begin{aligned}
& \int_{\Omega} \frac{\partial \bar{v}_{i}}{\partial x_{j}} \sigma_{i j}^{(1)} \mathrm{d} \boldsymbol{x}=\eta \int_{\Omega} \frac{\partial \bar{v}_{i}}{\partial x_{j}}\left(\frac{\partial v_{i}^{(1)}}{\partial x_{j}}+\frac{\partial v_{j}^{(1)}}{\partial x_{i}}\right) \mathrm{d} \boldsymbol{x} \\
& +\left(\zeta-\frac{2}{3} \eta\right) \int_{\Omega} \delta_{i j} \frac{\partial \bar{v}_{i}}{\partial x_{j}} \frac{\partial v_{k}^{(1)}}{\partial x_{k}} \mathrm{~d} \boldsymbol{x}-\int_{\Omega} \delta_{i j} \frac{\partial \bar{v}_{i}}{\partial x_{j}} p^{(1)} \mathrm{d} \boldsymbol{x} .
\end{aligned}
$$

In order to unify the presentation of the spatial discretization process, it is convenient to use a more general (and abstract) notation of the variational formulation. In principle, the weak form can be interpreted as the sum of different bilinear mappings (which will also referred to as dual pairs) of the type

$$
\langle\cdot, \cdot\rangle: V^{g *} \times V^{g} \rightarrow \mathbb{R}
$$

where $V^{g}$ denotes the underlying function space with regard to the field variable $g$ and $V^{g *}$ represents its associated dual space. Thereby, the first argument (the element of the dual space) arises from a linear mapping $K^{g h} \in L\left(V^{h}, V^{g *}\right), H^{g h} \in L\left(V^{\dot{h}}, V^{g *}\right)$ or $M^{g h} \in L\left(V^{\ddot{h}}, V^{g *}\right)$ that will be applied on a function $h \in V^{h}$ or on its first or second time derivative respectively. In conformity with this notation, the variational formulation 3.5 , 
which is expanded by the use of equation 3.6, can therefore be rewritten as

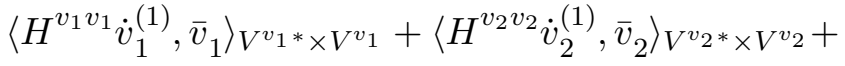

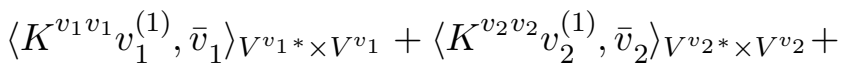

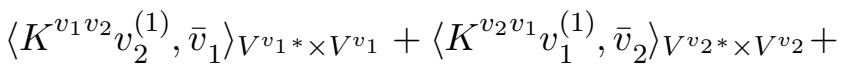

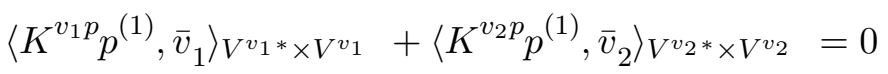

where the subscript of each dual pair indicates the respective spaces that are used by the bilinear mapping. The individual function spaces are specified in chapter 3.3.

The first order equation that describes the balance of mass must also be converted into its variational formulation, which is given by

$$
\frac{1}{c_{0}^{2}} \int_{\Omega} \bar{p} \frac{\partial p^{(1)}}{\partial t} \mathrm{~d} \boldsymbol{x}+\rho^{(0)} \int_{\Omega} \bar{p} \frac{\partial v_{i}^{(1)}}{\partial x_{i}} \mathrm{~d} \boldsymbol{x}=0 .
$$

In the same way as described above, the variational form of the mass balance can be represented - by using the dual pair notation - as

$$
\left\langle H^{p p} \dot{p}^{(1)}, \bar{p}\right\rangle_{V^{p *} \times V^{p}}+\left\langle K^{p v_{1}} v_{1}^{(1)}, \bar{p}\right\rangle_{V^{p *} \times V^{p}}+\left\langle K^{p v_{2}} v_{2}^{(1)}, \bar{p}\right\rangle_{V^{p *} \times V^{p}}=0 .
$$

Basilar Membrane In this work, the passive dynamics of the cochlear partition are represented by the differential equation 2.30. Its variational formulation can be evaluated to

$$
\begin{aligned}
& \int_{\Gamma_{\mathrm{bm}}} m \bar{\xi} \frac{\partial^{2} \xi}{\partial t^{2}} \mathrm{~d} x+\int_{\Gamma_{\mathrm{bm}}} \eta_{\text {lon }} A \frac{\partial \bar{\xi}}{\partial x} \frac{\partial^{2} \xi}{\partial x \partial t} \mathrm{~d} x+ \\
& \int_{\Gamma_{\mathrm{bm}}} h_{e} \bar{\xi} \frac{\partial \xi}{\partial t} \mathrm{~d} x+\int_{\Gamma_{\mathrm{bm}}} k \bar{\xi} \xi \mathrm{d} x=\int_{\Gamma_{\mathrm{bm}}} \bar{\xi} f \mathrm{~d} x
\end{aligned}
$$

where the force-term on the right hand side can be written as

$$
\begin{aligned}
& \int_{\Gamma_{\mathrm{bm}}} \bar{\xi} f \mathrm{~d} x=\left.\int_{\Gamma_{\mathrm{bm}}} w_{b m} \bar{\xi} p^{(1)}\right|_{\boldsymbol{x}=(x,+0)} \mathrm{d} x \\
& \quad-\left.\int_{\Gamma_{\mathrm{bm}}} w_{b m} \bar{\xi} p^{(1)}\right|_{\boldsymbol{x}=(x,-0)} \mathrm{d} x+\int_{\Gamma_{\mathrm{bm}}} \alpha \beta \bar{\xi} \zeta \mathrm{d} x .
\end{aligned}
$$

Similar to above, the weak form can now be transformed into a generalized statement, which consist of different dual pairs:

$$
\begin{aligned}
& \left\langle M^{\xi \xi} \ddot{\xi}, \bar{\xi}\right\rangle_{V^{\xi *} \times V^{\xi}}+\left\langle H^{\xi \xi} \dot{\xi}, \bar{\xi}\right\rangle_{V^{\xi * \times} \times V^{\xi}}+ \\
& \left\langle K^{\xi \xi} \xi, \bar{\xi}\right\rangle_{V^{\xi *} \times V^{\xi}}+\left\langle K^{\xi p} p^{(1)}, \bar{\xi}\right\rangle_{V^{\xi *} \times V^{\xi}}+ \\
& \left\langle K^{\xi \zeta} \zeta, \bar{\xi}\right\rangle_{V^{\xi *} \times V^{\xi}}=0 .
\end{aligned}
$$


Outer Hair Cell Motility The additional force $\vartheta$ that might be applied by the outerhair-cell motility and that acts as an additional load on the basilar membrane can be specified on the basis of the hair bundle deflection described by equation 2.36. According to the strategy mentioned above, the weak form of this differential equation can be calculated to

$$
\begin{gathered}
\int_{\Gamma_{\mathrm{bm}}} m_{\mathrm{tm}} \bar{\zeta} \frac{\partial^{2} \zeta}{\partial t^{2}} \mathrm{~d} x+\int_{\Gamma_{\mathrm{bm}}} h_{\mathrm{tm}} \bar{\zeta} \frac{\partial \zeta}{\partial t} \mathrm{~d} x+\int_{\Gamma_{\mathrm{bm}}} k_{\mathrm{tm}} \bar{\zeta} \zeta \mathrm{d} x= \\
\int_{\Gamma_{\mathrm{bm}}} g_{\mathrm{tm}} m_{\mathrm{tm}} \bar{\zeta} \frac{\partial^{2} \xi}{\partial t^{2}} \mathrm{~d} x .
\end{gathered}
$$

If each integral is understood as a bilinear form, the variational formulation can be written as

$$
\begin{aligned}
& \left\langle M^{\zeta \zeta} \ddot{\zeta}, \bar{\zeta}\right\rangle_{V^{\zeta * \times V}}+\left\langle H^{\zeta \zeta} \dot{\zeta}, \bar{\zeta}\right\rangle_{V^{\zeta *} \times V^{\zeta}}+ \\
& \left\langle K^{\zeta \zeta} \zeta, \bar{\zeta}\right\rangle_{V^{\zeta *} \times V^{\zeta}}+\left\langle M^{\zeta \xi} \ddot{\xi}, \bar{\zeta}\right\rangle_{V^{\zeta * \times V} V^{\zeta}}=0 .
\end{aligned}
$$

It is important to note that, in contrast to the fluid-viscosity coefficients $\eta$ and $\zeta$ and the small signal sound speed $c_{0}$ which are assumed to be constant in the present study, most of the coefficients, used for the representation of the basilar membrane and the outer hair cell motility, are assumed to vary along the longitudinal axis. As a consequence, the non-constant coefficients must be explicitly taken into account by performing a numerical quadrature (cf. chapter 3.4.4).

\subsubsection{The Second Order System}

Similar to the first order problem, the fluid-dynamics of the acoustic streaming subproblem can be converted into a variational formulation by using the mean second order equation of the momentum conservation 2.48, Its variational formulation is given by

$$
\begin{gathered}
\int_{\Omega} \delta_{i j} \frac{\partial \bar{v}_{i}}{\partial x_{j}} p^{(d c)} \mathrm{d} \boldsymbol{x}+\eta \int_{\Omega} \frac{\partial \bar{v}_{i}}{\partial x_{j}}\left(\frac{\partial v_{i}^{(d c)}}{\partial x_{j}}+\frac{\partial v_{j}^{(d c)}}{\partial x_{i}}\right) \mathrm{d} \boldsymbol{x} \\
+\left(\zeta-\frac{2}{3} \eta\right) \int_{\Omega} \delta_{i j} \frac{\partial \bar{v}_{i}}{\partial x_{j}} \frac{\partial v_{k}^{(d c)}}{\partial x_{k}} \mathrm{~d} \boldsymbol{x}=\int_{\Omega} \bar{v}_{i} \tilde{f}_{i} \mathrm{~d} \boldsymbol{x} .
\end{gathered}
$$

The generalized formulation of this weak form can be written as

$$
\begin{aligned}
& \left\langle G^{v_{1} v_{1}} v_{1}^{(d c)}, \bar{v}_{1}\right\rangle_{W^{v_{1} *} \times W^{v_{1}}}+\left\langle G^{v_{2} v_{2}} v_{2}^{(d c)}, \bar{v}_{2}\right\rangle_{W^{v_{2} *} \times W^{v_{2}}}+ \\
& \left\langle G^{v_{1} v_{2}} v_{2}^{(d c)}, \bar{v}_{1}\right\rangle_{W^{v_{1} *} \times W^{v_{1}}}+\left\langle G^{v_{2} v_{1}} v_{1}^{(d c)}, \bar{v}_{2}\right\rangle_{W^{v_{2} *} \times W^{v_{2}}}+ \\
& \left\langle G^{v_{1} p} p^{(d c)}, \bar{v}_{1}\right\rangle_{W^{v_{1} *} \times W^{v_{1}}}+\left\langle G^{v_{2} p} p^{(d c)}, \bar{v}_{2}\right\rangle_{W^{v_{2} *} \times W^{v_{2}}}= \\
& \left\langle S^{v_{1}}, \bar{v}_{1}\right\rangle_{W^{v_{1} *} \times W^{v_{1}}}+\left\langle S^{v_{2}}, \bar{v}_{2}\right\rangle_{W^{v_{2} *} \times W^{v_{2}}}
\end{aligned}
$$


where a set of different dual pairs need to be introduced. The force-source-distribution $\tilde{f}$ is represented by the dual elements $S^{v_{i}} \in W^{v_{i} *}$.

The weak form of the mean second order equation 2.46, which was originally deduced from the balance of mass equation, can be evaluated to

$$
\rho^{(0)} \int_{\Omega} \bar{p} \frac{\partial v_{i}^{(d c)}}{\partial x_{i}} \mathrm{~d} \boldsymbol{x}=\int_{\Omega} \bar{p} \tilde{m} \mathrm{~d} \boldsymbol{x} .
$$

According to the other weak forms, this variational formulation can also be converted into its uniform version

$$
\left\langle G^{p v_{1}} v_{1}^{(d c)}, \bar{p}\right\rangle_{W^{p *} \times W^{p}}+\left\langle G^{p v_{2}} v_{2}^{(d c)}, \bar{p}\right\rangle_{W^{p *} \times W^{p}}=\left\langle S^{p}, \bar{p}\right\rangle_{W^{p *} \times W^{p}}
$$

where $S^{p} \in W^{p *}$ is associated with the mass-source-distribution $\tilde{m}$.

\subsection{Function Spaces}

\subsubsection{Spatial Solution Spaces}

The construction of the finite element spaces must be based on the structure of the correct solution spaces of the individual field variables. This work uses an approach that applies different discretization techniques with respect to the spatial and the temporal variables. For the examination of the spatial solution space, the time variable is assumed to be fixed to a specific time. Then, the actual state of the system can be described on the basis of solution spaces that only take the spatial dimensions into account. For a more generalized consideration of the solution spaces that also take the time-dependence into account see e.g. [24].

Due to the composition of the individual bilinear forms that constitute the variational formulation, it seems to be obvious to define the solution space as the space that consists of all smooth functions $f \in C^{\infty}(\Omega)$ of which all partial derivatives up to an order $k$ as well as the function itself are square-integrable functions (and therefore a member of the Lebesgue-space $L^{2}(\Omega)$ ). In doing so, the number $k$ depends on the order of derivatives that can be found within their associated bilinear forms. But it has been shown that this function space is too small for the representation of solutions that arise from problems that are described by a variational formulation of partial differential equations. (cf. e.g. [23]) From the topological point of view, the correct solution space can be established by the completion of this function space with respect to the so-called Sobolev-norm, which can be written as

$$
\|g\|_{k}:=\left(\sum_{|\alpha| \leq k}\left\|\partial^{\alpha} g\right\|_{0}\right)^{\frac{1}{2}}
$$


The superscript $\alpha$ is associated with the multi-index notation which allows a simple representation of different differential operators. This function space is well-known as Sobolevspace $H^{k}$ and since the Sobolev-space is complete with respect to the norm $\|\cdot\|_{k}$, it constitutes a Banach space. The functions of the Sobolev-space can be characterized by its weak derivatives. In this context, a function $h \in L^{2}(\Omega)$ is referred to as weak derivative with respect to the multi-index $\alpha$ of the function $g \in L^{2}(\Omega)$, if

$$
\int_{\Omega} g \varphi \mathrm{d} \boldsymbol{x}=(-1)^{|\alpha|} \int_{\Omega} h \partial^{\alpha} \varphi \mathrm{d} \boldsymbol{x}
$$

holds for all $\varphi \in C_{0}^{\infty}(\Omega)$. Then, it can be shown (cf. e.g. [23]) that a function is an element of the Sobolev-space $H^{k}$ if and only if its weak derivatives up to the order $k$ and the function itself are elements of the Lebesgue space $L^{2}(\Omega)$.

The function spaces of the individual field variables can be identified on the basis of the resultant bilinear forms that are constituted by the variational formulations. By taking the specific boundary conditions into account, the function spaces of the longitudinal velocity component are given by

$$
\begin{aligned}
V^{v_{1}}= & \left\{v_{1} \in H^{1}(\Omega) \mid v_{1}(\boldsymbol{x})=\frac{\partial \xi_{\mathrm{ow}}}{\partial t} \text { for all } \boldsymbol{x} \in \Gamma_{\mathrm{ow}},\right. \\
& \left.v_{1}(\boldsymbol{x})=0 \text { for all } \boldsymbol{x} \in \Gamma_{\mathrm{r}} \cup \Gamma_{\mathrm{bm}+} \cup \Gamma_{\mathrm{bm}-}\right\}, \\
V^{\dot{v}_{1}}= & L^{2}(\Omega) .
\end{aligned}
$$

In the context of the Sobolev-spaces, a specification of function values at the boundary does not make sense, since the $d$-dimensional Lebesgue measure of the boundary is zero. In order to overcome this difficulty, it has been shown (cf. e.g. [23]) that under certain conditions regarding the domain $\Omega$, a $H^{1}(\Omega)$-function can be uniquely extended by means of the linear trace operator

$$
\mathrm{T}:\left(H^{1}(\Omega),\|\cdot\|_{1}\right) \rightarrow\left(L^{2}(\delta \Omega),\|\cdot\|_{0}\right) .
$$

Therefore, if the function-values at the boundary domain are interpreted as a squareintegrable and $d-1$-measurable function, the Dirichlet-condition can be established by using the trace-operator T1 But for reasons of simplicity, the trace operator will not be explicitly stated in the context of the declaration of the Dirichlet conditions.

By adapting the respective boundary conditions, the function spaces of the vertical

\footnotetext{
${ }^{1}$ In principle, the trace theorem can not be applied on the cochlea domain in its current form, since the domain does not fulfill the requirements of a so-called Lipschitz domain. But the domain can be simply transformed into a Lipschitz domain by inserting a thin gap between the upper boundary of the basilar membrane and its lower counterpart.
} 
velocity component can be written as

$$
\begin{aligned}
& V^{v_{2}}=\left\{v_{2} \in H^{1}(\Omega) \mid v_{2}(\boldsymbol{x})=\frac{\partial \xi}{\partial t} \text { for all } \boldsymbol{x} \in \Gamma_{\mathrm{bm}+} \cup \Gamma_{\mathrm{bm}-},\right. \\
& \left.v_{1}(\boldsymbol{x})=0 \text { for all } \boldsymbol{x} \in \Gamma_{\mathrm{r}} \cup \Gamma_{\mathrm{ow}} \cup \Gamma_{\mathrm{rw}}\right\} \\
& V^{\dot{v}_{2}}=L^{2}(\Omega) .
\end{aligned}
$$

Since the integrals of the variational formulations that are associated with the pressure do not contain any spatial derivatives of the pressure variable, the pressure field as well as its time-derivative can be represented by an element of the function space

$$
V^{p}=V^{\dot{p}}=L^{2}(\Omega) \text {. }
$$

So far, only the function spaces of the fields that describe the fluid dynamics have been considered. In a similar way, also function spaces of the fields that are associated with the basilar membrane displacement and hair bundle deflection can be introduced. In contrast to the velocity and the pressure field, the domain of the functions that are associated with the dynamics of the basilar membrane is a one-dimensional sub-space. On the basis of the weak form 3.11, the function spaces of the basilar membrane displacement and its temporal derivatives are given by

$$
\begin{aligned}
V^{\xi}=V^{\ddot{\xi}} & =L^{2}\left(\Gamma_{\mathrm{bm}}\right), \\
V^{\dot{\xi}} & =H^{1}\left(\Gamma_{\mathrm{bm}}\right) .
\end{aligned}
$$

The variational formulation of the differential equation that describes the hair bundle deflection implies that the respective function spaces can be written as

$$
V^{\zeta}=V^{\dot{\zeta}}=V^{\ddot{\zeta}}=L^{2}\left(\Gamma_{\mathrm{bm}}\right)
$$

The acoustic streaming subproblem constitutes a stationary problem. Therefore, the solution does not depend on the time. On the basis of the variational formulation, the function spaces of the velocity components can be written as

$$
\begin{aligned}
& W^{v_{1}}=\left\{v_{1} \in H^{1}(\Omega) \mid v_{1}(\boldsymbol{x})=v_{1}^{(d c)} \text { for all } \boldsymbol{x} \in \Gamma\right\}, \\
& W^{v_{2}}=\left\{v_{2} \in H^{1}(\Omega) \mid v_{2}(\boldsymbol{x})=v_{2}^{(d c)} \text { for all } \boldsymbol{x} \in \Gamma\right\}
\end{aligned}
$$

where the mean velocity component $\boldsymbol{v}^{(d c)}$ at the boundaries can be evaluated from the boundary condition 2.52 which bases on the results of the first order acoustic subproblem. Similar to the acoustic system, the variational formulation of the secondary system does not consist on spatial derivatives of the pressure variable. Therefore, the pressure can be represented by an element of the function space

$$
W^{p}=L^{2}(\Omega) .
$$




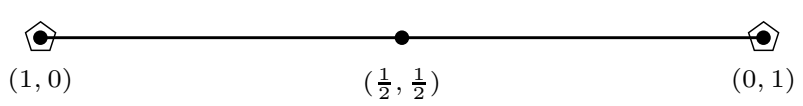

(a)

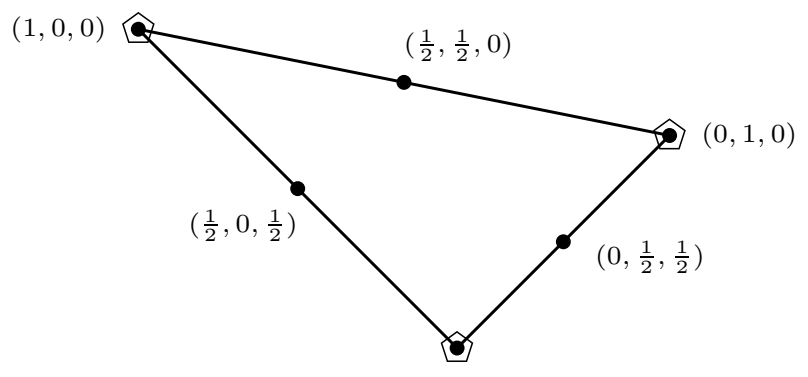

$(0,0,1)$

(b)

Figure 3.3: Arrangement of the local nodes and their respective barycentric coordinates on (a) linesegments as well as (b) triangular elements. Nodes that are associated with function spaces of polynomials of degree at most two are marked by black dots. The hollow pentagons indicate the nodes in the case of function spaces that are constructed on the basis of polynomials of degree at most one.

\subsubsection{Local Finite Element Spaces}

As part of the discretization process the infinite-dimensional solution spaces (as specified above) have to be replaced by appropriate finite-dimensional subspaces. The usual approach for the construction of such finite-dimensional subspaces in the context of the finite element method is based on finite-dimensional local function spaces which are defined on each individual simplex of the mesh. These local function spaces may then be combined in such a way that the resultant global finite-dimensional space is a subspace of the solution space.

In most cases, the field variables on a single simplex can be approximated with sufficient accuracy by using polynomials of degree at most a number $d$. It is quite obvious that such a polynomial (restricted to a single simplex $S$ ) is an element of the Sobolev-Space $H^{1}(S)$. It has been shown (cf. e.g. [23]) that a global function composed of individual local polynomials is an element of the Sobolev-Space $H^{1}(M)$ if and only if the global function is a continuous function on $M$. Therefore, the overall function does not need to be continuously differentiable across the edges of the simplices. In contrast to the other variables, the pressure is an element of the less restrictive space $L^{2}$. As a consequence, the global function space that is used for the approximation of the pressure field does not even need to be continuous across the simplex-borders.

Of course, it would be possible to specify each local polynomial by using its respective coefficients as degrees of freedom. But this approach would not be very useful, since the global continuity condition can not be directly taken into account. In the context of the finite element method, the local functions are usually determined by the specification of its 


\begin{tabular}{ll}
\hline Associated Node & Local Basis Function \\
\hline $\boldsymbol{\beta}_{(\mathrm{bar})}^{0}=(1,0)$ & $L_{(\mathrm{bar})}^{0}\left(S_{(\mathrm{bar})}^{1}\right):=\beta_{0}$ \\
$\boldsymbol{\beta}_{(\mathrm{bar})}^{1}=(0,1)$ & $L_{(\mathrm{bar})}^{1}\left(S_{(\mathrm{bar})}^{1}\right):=\beta_{1}$ \\
\hline
\end{tabular}

Table 3.1: Basis functions on line segments that span the space of polynomials of degree at most one (cf. [42]). Each basis function can be associated with a certain node where it doesn't vanish.

function values and/or spatial derivatives at a number of individual distinct points (also referred to as nodes) within the simplex.

Since all field variables that are needed in the context of the cochlear model presented here do not need to be continuously differentiable at the edges of the simplices, it is sufficient to use elements of the well-known Lagrange family. The Lagrange elements are characterized by solving the interpolation problem that specifies only the function values (and not the spatial derivatives) at each node. There are two properties that are essential for the application of the Lagrange elements in the context of the finite element method:

(1) The polynomial is well-defined by the function values at the nodes of a certain simplex.

(2) The polynomial restricted to any sub-simplex is well-defined by the function values at the nodes of the certain sub-simplex.

The interpolation functions of the Lagrange family are polynomials of up to degree $k$. The space of these polynomials can be naturally identified with the Euclidean space $\mathbb{R}^{p}$, where the dimension $p$ of this space is given by the binomial coefficient $\left(\begin{array}{c}d+k \\ k\end{array}\right)$. The interpolation problem can now be interpreted as a mapping from the Euclidean space $\mathbb{R}^{q}$, which serves as a representation of the function values at the $q$ nodes, into the polynomial space mentioned above. In this context, it becomes apparent that the number of nodes must not exceed the dimension of the polynomial space in order to ensure that the interpolation problem is solvable and furthermore that this solution is also well-defined. On the basis of the declaration of a set of polynomials $N^{i}$ that belong to the Lagrange family and that are characterized by the condition

$$
N^{i}\left(n^{j}\right)=\delta_{i j}
$$

for all nodes $n^{j}$ the mapping can be simply specified by an appropriate linear combination of these basis functions. In this case, the resultant interpolation function also complies with property (1), since this kind of mapping ensures the existence as well as the uniqueness of the interpolating polynomial. By the restriction on an arbitrary sub-simplex, the same line of argument can be used to show property (2). (cf. [23])

In this work, polynomials of degree at most one (for the force-like variables) and at most two (for velocity or displacement-like variables) are used. In order to distinguish both types of local function spaces with regard to their maximal polynomial degree, the respective space will be referred to as linear or quadratic. The arrangement of the nodes 


\begin{tabular}{ll}
\hline Associated Node & Local Basis Function \\
\hline $\boldsymbol{\beta}_{(\mathrm{bar})}^{0}=(1,0)$ & $Q_{(\mathrm{bar})}^{0}\left(S_{(\mathrm{bar})}^{1}\right):=\left(2 \beta_{0}-1\right) \beta_{0}$ \\
$\boldsymbol{\beta}_{(\mathrm{bar})}^{1}=(0,1)$ & $Q_{(\mathrm{bar})}^{1}\left(S_{(\mathrm{bar})}^{1}\right):=\left(2 \beta_{1}-1\right) \beta_{1}$ \\
$\boldsymbol{\beta}_{(\mathrm{bar})}^{2}=\left(\frac{1}{2}, \frac{1}{2}\right)$ & $Q_{(\mathrm{bar})}^{2}\left(S_{(\mathrm{bar})}^{1}\right):=4 \beta_{0} \beta_{1}$ \\
\hline
\end{tabular}

Table 3.2: Basis functions on line segments that span the space of polynomials of degree at most two (cf. [42]). Each basis function can be associated with a certain node where it doesn't vanish.

\begin{tabular}{ll}
\hline Associated Node & Local Basis Function \\
\hline $\boldsymbol{\beta}_{(\mathrm{bar})}^{0}=(1,0,0)$ & $L_{(\mathrm{bar})}^{0}\left(S_{(\mathrm{bar})}^{2}\right):=\beta_{0}$ \\
$\boldsymbol{\beta}_{(\mathrm{bar})}^{1}=(0,1,0)$ & $L_{(\mathrm{bar})}^{1}\left(S_{(\mathrm{bar})}^{2}\right):=\beta_{1}$ \\
$\boldsymbol{\beta}_{(\mathrm{bar})}^{2}=(0,0,1)$ & $L_{(\mathrm{bar})}^{2}\left(S_{(\mathrm{bar})}^{2}\right):=\beta_{2}$ \\
\hline
\end{tabular}

Table 3.3: Basis functions on triangular elements that span the space of polynomials of degree at most one (cf. [42]). Each basis function can be associated with a certain node where it doesn't vanish.

in the linear case as well as the quadratic case for both line-segments and triangles is illustrated in figure 3.3. The respective set of basis functions that comply with equation 3.34 are listed in the tables $3.1,3.2,3.3$ and 3.4 with respect to the barycentric reference system.

Each linear and quadratic basis function with respect to a specific simplex is then defined as

$$
\begin{aligned}
L^{i}\left(S^{d}\right) & :=L_{(\mathrm{bar})}^{i}\left(S_{(\mathrm{bar})}^{d}\right) \circ \boldsymbol{\beta}(\boldsymbol{x}), \\
Q^{i}\left(S^{d}\right) & :=Q_{(\mathrm{bar})}^{i}\left(S_{(\mathrm{bar})}^{d}\right) \circ \boldsymbol{\beta}(\boldsymbol{x}) .
\end{aligned}
$$

The local function spaces for the linear and the quadratic case are now given by

$$
\begin{aligned}
& \mathcal{L}\left(S^{d}\right):=\operatorname{span}\left\{L^{i}\left(S^{d}\right) \mid i=1,2, \ldots\right\}, \\
& \mathcal{Q}\left(S^{d}\right):=\operatorname{span}\left\{Q^{i}\left(S^{d}\right) \mid i=1,2, \ldots\right\} .
\end{aligned}
$$

\subsubsection{Global Finite Element Spaces}

As mentioned above, it is necessary to ensure that the global functions are continuous across the edges of the simplices in order to guarantee that the Sobolev-spaces contain the resultant functions. In terms of a mathematical formulation, the overall linear function space with respect to the fluid-domain can be stated as

$$
\begin{aligned}
\mathcal{L}\left(M^{\Omega}\right): & =\left\{f \in C^{0}\left(M^{\Omega}\right)\left|\forall S^{2} \in M^{\Omega}: f\right|_{S^{2}} \in \mathcal{L}\left(S^{2}\right)\right\} \\
& =\operatorname{span}\left\{L_{1}^{\Omega}, L_{2}^{\Omega}, \ldots, L_{\operatorname{dim} \mathcal{L}\left(M^{\Omega}\right)}^{\Omega}\right\}
\end{aligned}
$$




\begin{tabular}{ll}
\hline Associated Node & Local Basis Function \\
\hline $\boldsymbol{\beta}_{(\text {bar })}^{0}=(1,0,0)$ & $Q_{(\text {bar })}^{0}\left(S_{(\text {bar })}^{2}\right):=\left(2 \beta_{0}-1\right) \beta_{0}$ \\
$\boldsymbol{\beta}_{(\text {bar })}^{1}=(0,1,0)$ & $Q_{(\text {bar })}^{1}\left(S_{(\text {bar })}^{2}\right):=\left(2 \beta_{1}-1\right) \beta_{1}$ \\
$\boldsymbol{\beta}_{(\text {bar })}^{2}=(0,0,1)$ & $Q_{(\text {bar })}^{2}\left(S_{(\text {bar })}^{2}\right):=\left(2 \beta_{2}-1\right) \beta_{2}$ \\
$\boldsymbol{\beta}_{\text {(bar) }}^{3}=\left(0, \frac{1}{2}, \frac{1}{2}\right)$ & $Q_{(\text {bar })}^{3}\left(S_{(\text {bar })}^{2}\right):=4 \beta_{1} \beta_{2}$ \\
$\boldsymbol{\beta}_{(\text {bar })}^{4}=\left(\frac{1}{2}, 0, \frac{1}{2}\right)$ & $Q_{(\text {bar })}^{4}\left(S_{(\text {bar })}^{2}\right):=4 \beta_{0} \beta_{2}$ \\
$\boldsymbol{\beta}_{(\text {bar })}^{5}=\left(\frac{1}{2}, \frac{1}{2}, 0\right)$ & $Q_{(\text {bar })}^{5}\left(S_{(\text {bar })}^{2}\right):=4 \beta_{0} \beta_{1}$ \\
\hline
\end{tabular}

Table 3.4: Basis functions on triangular elements that span the space of polynomials of degree at most two (cf. [42]). Each basis function can be associated with a certain node where it doesn't vanish.

where the functions $L_{i}^{\Omega}$ establish a basis of the global function space and will be specified below. In the same way, the overall quadratic function space can be represented by

$$
\begin{aligned}
\mathcal{Q}\left(M^{\Omega}\right): & =\left\{f \in C^{0}\left(M^{\Omega}\right)\left|\forall S^{2} \in M^{\Omega}: f\right|_{S^{2}} \in \mathcal{Q}\left(S^{2}\right)\right\} \\
& =\operatorname{span}\left\{Q_{1}^{\Omega}, Q_{2}^{\Omega}, \ldots, Q_{\operatorname{dim} \mathcal{Q}\left(M^{\Omega}\right)}^{\Omega}\right\}
\end{aligned}
$$

The continuity condition involves the necessity that all function values at those local nodes that are positioned at the same location but belong to different simplices are identical. Then, the continuity condition can be guaranteed due to the interpolation condition (2) as mentioned above. Therefore, it is useful to join these adjacent local nodes to a common global node. From a mathematical point of view, a global node constitutes an equivalence class of the set of all local nodes of a mesh. In this context, two nodes are members of the same equivalence class if and only if both nodes can be represented by identical barycentric coordinates with respect to a common sub-simplex (to which both nodes belong).

For the implementation, it is useful to label each global node and each global basis function by an index. Therefore, let

$$
\begin{aligned}
\mathrm{I}^{\mathcal{L}\left(M^{\Omega}\right)} & :=\left\{i \in \mathbb{N} \mid 1 \leq i \leq \operatorname{dim} \mathcal{L}\left(M^{\Omega}\right)\right\}, \\
\mathrm{I}^{\mathcal{Q}\left(M^{\Omega}\right)} & :=\left\{i \in \mathbb{N} \mid 1 \leq i \leq \operatorname{dim} \mathcal{Q}\left(M^{\Omega}\right)\right\}
\end{aligned}
$$

be the index-families that are associated with the global linear and the global quadratic function spaces. If each global node is uniquely labelled by such an index, the basis functions $L_{i}^{\Omega}$ or $Q_{i}^{\Omega}$ respectively of the global function spaces can be defined indirectly by

$$
\begin{array}{ll}
L_{i}^{\Omega}\left(n_{j}\right)=\delta_{i j} & \text { for all } j \in \mathrm{I}^{\mathcal{L}\left(M^{\Omega}\right)}, \\
Q_{i}^{\Omega}\left(n_{j}\right)=\delta_{i j} & \text { for all } j \in \mathrm{I}^{\mathcal{Q}\left(M^{\Omega}\right)}
\end{array}
$$

with $n_{j}$ being the global node that acts as a representative for all local nodes that belong to this global node. The uniqueness of these basis-functions is a direct consequence of the interpolation condition (1). In the same way, similar global function spaces with regard to 
the basilar membrane domain can be established. The function space of piecewise linear functions on individual line-segments of the basilar membrane is given by

$$
\begin{aligned}
\mathcal{L}\left(M^{\Gamma_{\mathrm{bm}}}\right): & =\left\{f \in C^{0}\left(M^{\Gamma_{\mathrm{bm}}}\right)\left|\forall S^{1} \in M^{\Gamma_{\mathrm{bm}}}: f\right|_{S^{1}} \in \mathcal{L}\left(S^{1}\right)\right\} \\
& =\operatorname{span}\left\{L_{1}^{\Gamma_{\mathrm{bm}}}, L_{2}^{\Gamma_{\mathrm{bm}}}, \ldots, L_{\operatorname{dim} \mathcal{L}\left(M^{\Gamma_{\mathrm{bm}}}\right)}^{\Gamma_{\mathrm{bm}}}\right\} .
\end{aligned}
$$

The function space that uses piecewise quadratic polynomials can be written as

$$
\begin{aligned}
\mathcal{Q}\left(M^{\Gamma_{\mathrm{bm}}}\right): & =\left\{f \in C^{0}\left(M^{\Gamma_{\mathrm{bm}}}\right)\left|\forall S^{1} \in M^{\Gamma_{\mathrm{bm}}}: f\right|_{S^{1}} \in \mathcal{Q}\left(S^{1}\right)\right\} \\
& =\operatorname{span}\left\{Q_{1}^{\Gamma_{\mathrm{bm}}}, Q_{2}^{\Gamma_{\mathrm{bm}}}, \ldots, Q_{\operatorname{dim} \mathcal{Q}\left(M^{\Gamma_{\mathrm{bm}}}\right)}^{\Gamma_{\mathrm{bm}}}\right\} .
\end{aligned}
$$

As in the case of the global nodes that are associated with the fluid domain, each global node of the basilar membrane domain is indicated by a unique number. The indices of a global node and the basis functions are chosen in such a way that the following relations hold:

$$
\begin{array}{ll}
L_{i}^{\Gamma_{\mathrm{bm}}}\left(n_{j}\right)=\delta_{i j} & \text { for all } j \in \mathrm{I}^{\mathcal{L}\left(M^{\left.\Gamma_{\mathrm{bm}}\right)}\right.}, \\
Q_{i}^{\Gamma_{\mathrm{bm}}}\left(n_{j}\right)=\delta_{i j} & \text { for all } j \in \mathrm{I}^{\mathcal{Q}\left(M^{\left.\Gamma_{\mathrm{bm}}\right)}\right.} .
\end{array}
$$

For the assembly of the matrices that are used to represent the discretized system it is important to use an increasing set of numbers for the index families used in the previous equations. Therefore, these index families are given by

$$
\begin{aligned}
\mathrm{I}^{\mathcal{L}\left(M^{\Gamma \mathrm{bm}}\right)}: & =\left\{i \in \mathbb{N} \mid 1 \leq i \leq \operatorname{dim} \mathcal{L}\left(M^{\Gamma_{\mathrm{bm}}}\right)\right\}, \\
\mathrm{I}^{\mathcal{Q}\left(M^{\Gamma \mathrm{bm}}\right)}: & =\left\{i \in \mathbb{N} \mid 1 \leq i \leq \operatorname{dim} \mathcal{Q}\left(M^{\Gamma_{\mathrm{bm}}}\right)\right\} .
\end{aligned}
$$

Furthermore, it is useful to combine the respective basis functions of the global finite element spaces $L_{i}^{\Omega}, Q_{i}^{\Omega}, L_{i}^{\Gamma_{\mathrm{bm}}}$ and $Q_{i}^{\Gamma_{\mathrm{bm}}}$ into the common vectors $\mathbf{L}^{\Omega}, \mathbf{Q}^{\Omega}, \mathbf{L}^{\Gamma_{\mathrm{bm}}}$ and $\mathbf{Q}^{\Gamma_{\mathrm{bm}}}$.

\subsection{Spatial Discretization}

\subsubsection{The First Order System}

Acoustic Subproblem In the last chapter the spatial solution spaces of the individual physical field variables have been introduced. As part of the discretization process, these infinite dimensional function spaces are replaced by the global finite element spaces, presented in chapter 3.3.3. In this context, the individual scalar solution functions are approximated by the linear combination of the respective basis functions. In this work, the two components of the velocity field as well as its first time derivatives are represented by 
the quadratic finite element functions $Q$ with respect to the two-dimensional fluid domain $\Omega$. Therefore, the individual velocity components can be written as

$$
\begin{array}{ll}
v_{1}^{(1)} \approx \hat{\mathbf{v}}_{1}^{(1)} \cdot \mathbf{Q}^{\Omega}, & v_{2}^{(1)} \approx \hat{\mathbf{v}}_{2}^{(1)} \cdot \mathbf{Q}^{\Omega}, \\
\dot{v}_{1}^{(1)} \approx \dot{\hat{\mathbf{v}}}_{1}^{(1)} \cdot \mathbf{Q}^{\Omega}, & \dot{v}_{2}^{(1)} \approx \dot{\hat{\mathbf{v}}}_{2}^{(1)} \cdot \mathbf{Q}^{\Omega},
\end{array}
$$

where the hat-symbol above a variable refers to a nodal vector. A single component of a nodal vector is characterized by its connection to a specific global node. According to the linear combination it becomes obvious that the function value at a specific node of the resultant approximation function is identical to the associated component of the nodal vector.

It has been shown that the right choice of the finite element spaces of the mixed system that takes the velocity field as well as the pressure field into account has a major influence on the stability, accuracy and convergence of the simulation process (cf. [36]). If the basis functions of the pressure field are, for example, chosen in such a way that their polynomial degree is identical to the order of the basis functions that are used to represent the velocity, the system will become - in a certain way - overconstrained. As a consequence, a significant spatial oscillation of the pressure function can be observed. In contrast, a stable system can be achieved by fulfilling the so-called Ladyzhenskaya-Babuska-Brezzi (LBB) condition, which can be written as

$$
\inf _{p \in V^{p} \backslash\{0\}} \sup _{\boldsymbol{v} \in \boldsymbol{V}^{v} \backslash\{\mathbf{0}\}} \frac{\left\langle K^{p v_{1}} v_{1}, p\right\rangle+\left\langle K^{p v_{2}} v_{2}, p\right\rangle}{\|p\|_{0}\|\boldsymbol{v}\|_{1}}>c
$$

where $\boldsymbol{V}^{v}=V^{v_{1}} \times V^{v_{2}}$ and $c>0$ is referred to a constant. The validity of the LBB condition has not only to be verified for functions of the underlying solution space (as e.g. shown by [24]), but also for the discretized finite element spaces. In order to ensure that the LBB-condition is also valid for the discretized finite element spaces, it has been shown that the maximal polynomial degree of the basis functions that are used for representing the pressure field has to be at least one order lower than the respective basis functions of the velocity components (cf. [36]). Therefore, since the velocity components are approximated by the use of quadratic basis functions, the function space of the pressure field is constituted by basis functions that are piecewise linear:

$$
p^{(1)} \approx \hat{\mathbf{p}}^{(1)} \cdot \mathbf{L}^{\Omega}, \quad \dot{p}^{(1)} \approx \dot{\hat{\mathbf{p}}}^{(1)} \cdot \mathbf{L}^{\Omega}
$$

Up to now, the discretized function spaces of the velocity fields do not consider the boundary conditions. The Dirichlet condition can be implemented by the specification of the respective function values at the boundary nodes. Therefore, it is useful to introduce a set 
of different index families in order to realize the boundary conditions:

$$
\begin{aligned}
& \mathrm{B}^{\mathcal{L}\left(M^{\Gamma \mathrm{bm}+}\right)} \subset \mathrm{I}^{\mathcal{L}\left(M^{\Omega}\right)}, \quad \mathrm{B}^{\mathcal{Q}\left(M^{\Gamma \mathrm{bm}+}\right)} \subset \mathrm{I}^{\mathcal{Q}\left(M^{\Omega}\right)},
\end{aligned}
$$

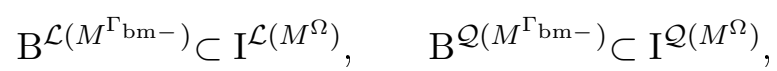

$$
\begin{aligned}
& \mathrm{B}^{\mathcal{L}\left(M^{\Gamma_{\mathrm{ow}}}\right)} \subset \mathrm{I}^{\mathcal{L}\left(M^{\Omega}\right)}, \quad \mathrm{B}^{\mathcal{Q}\left(M^{\Gamma \mathrm{ow}}\right)} \subset \mathrm{I}^{\mathcal{Q}\left(M^{\Omega}\right)}, \\
& \mathrm{B}^{\mathcal{L}\left(M^{\Gamma_{\mathrm{rw}}}\right)} \subset \mathrm{I}^{\mathcal{L}\left(M^{\Omega}\right)}, \quad \mathrm{B}^{\mathcal{Q}\left(M^{\Gamma_{\mathrm{rw}}}\right)} \subset \mathrm{I}^{\mathcal{Q}\left(M^{\Omega}\right)}, \\
& \mathrm{B}^{\mathcal{L}\left(M^{\Gamma_{\mathrm{r}}}\right)} \subset \mathrm{I}^{\mathcal{L}\left(M^{\Omega}\right)}, \quad \mathrm{B}^{\mathcal{Q}\left(M^{\Gamma_{\mathrm{r}}}\right)} \subset \mathrm{I}^{\mathcal{Q}\left(M^{\Omega}\right)} .
\end{aligned}
$$

Each family is a subset either of the linear or the quadratic finite element function space. An index $i$ is an element of one of these subsets if its associated node $n_{i}$ belongs to the respective boundary. Due to the construction of the mesh of the fluid domain in connection with the line-segments of the cochlear partition (cf. section 3.1.4), the nodes of the basilar membrane (in the linear as well as in the quadratic case) can be linked to the adjacent boundary nodes of the fluid domain. The various mappings from the nodes of the basilar membrane to the nodes that belong either to the upper duct or to the lower chamber of the cochlear model are illustrated by the following scheme:

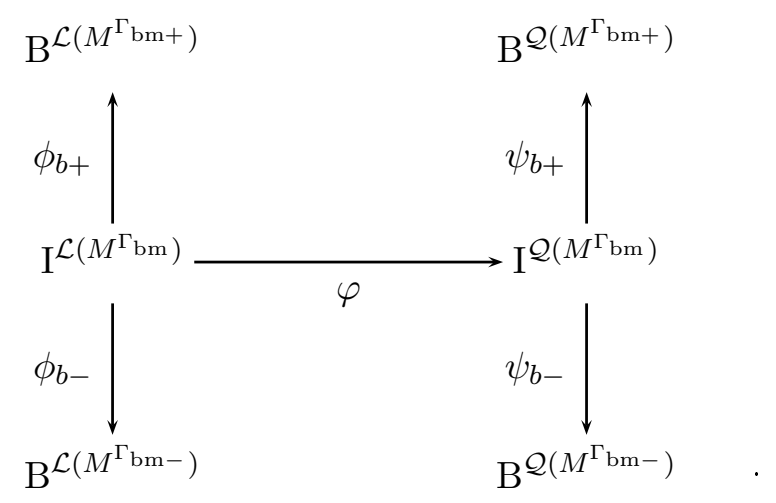

These mappings constitute bijections except of the function $\varphi$ which is only an injective function since the quadratic basis functions need additional nodes in the middle of each simplex. For reasons of simplicity, different sets of boundary nodes can be combined into a single set for which the Dirichlet condition must be applied. Since the Dirichlet conditions of the first velocity component differs from the second velocity component, two different sets of nodes have to be established:

$$
\begin{aligned}
& \mathrm{B}_{(1)}^{v_{1}}=\mathrm{B}^{\mathcal{Q}\left(M^{\Gamma_{\mathrm{ow}}}\right)} \cup \mathrm{B}^{\mathcal{Q}\left(M^{\Gamma_{\mathrm{bm}}+}\right)} \cup \mathrm{B}^{\mathcal{Q}\left(M^{\Gamma_{\mathrm{bm}}-}\right)} \cup \mathrm{B}^{\mathcal{Q}\left(M^{\Gamma_{\mathrm{r}}}\right)}, \\
& \mathrm{B}_{(1)}^{v_{2}}=\mathrm{B}^{\mathcal{Q}\left(M^{\Gamma_{\mathrm{ow}}}\right)} \cup \mathrm{B}^{\mathcal{Q}\left(M^{\Gamma_{\mathrm{rw}}}\right)} \cup \mathrm{B}^{\mathcal{Q}\left(M^{\Gamma_{\mathrm{bm}}}\right)} \cup \mathrm{B}^{\mathcal{Q}\left(M^{\Gamma_{\mathrm{bm}}-}\right)} \cup \mathrm{B}^{\mathcal{Q}\left(M^{\Gamma_{\mathrm{r}}}\right)} .
\end{aligned}
$$

As described in chapter 2.3.1, the acoustic subproblem is mainly characterized by the first order equation of the conservation of mass and by the first order equation of the conservation of linear momentum in combination with the specification of the stress tensor. This system of differential equations can be transformed into a semi-discretized system by 
using its variational formulation as introduced in chapter 3.2.1. As mentioned above, the system will initially be discretized with respect to the spatial dimensions. The temporal discretization process is described in chapter 3.5. The semi-discretized system can be represented by using a set of different matrices. These matrices can be assembled on the basis of the dual pairs as introduced in chapter 3.2. In doing this, each solution space has to be replaced by an appropriate finite element space. If the test-functions are successively substituted by different basis functions of the underlying finite element space and if the field variables are approximated by using equations 3.51 and 3.53, a linear system of equation can be established. Before determining the structure of this linear system of equation, the individual matrices should be introduced. In principle, the matrices can be associated with different terms of the original differential system of equation. The Newtonian fluid is characterized by the linear dependence between the stress tensor and the deformation tensor (cf. equation 2.7). This stress tensor is implemented in terms of the matrices $\mathbf{K}^{v_{1} v_{1}}$, $\mathbf{K}^{v_{2} v_{2}}, \mathbf{K}^{v_{1} v_{2}}, \mathbf{K}^{v_{2} v_{1}}, \mathbf{K}^{v_{1} p}$ and $\mathbf{K}^{v_{2} p}$. The first two matrices are given by the following specifications:

$$
\begin{aligned}
\mathrm{K}_{i j}^{v_{1} v_{1}} & = \begin{cases}\delta_{i j} & \text { if } i \in \mathrm{B}_{(1)}^{v_{1}} \\
\left\langle K^{v_{1} v_{1}} Q_{j}^{\Omega}, Q_{i}^{\Omega}\right\rangle_{\mathcal{Q}^{*} \times \mathcal{Q}} & \text { otherwise },\end{cases} \\
\mathrm{K}_{i j}^{v_{2} v_{2}} & = \begin{cases}\delta_{i j} & \text { if } i \in \mathrm{B}_{(1)}^{v_{2}} \\
\left\langle K^{v_{2} v_{2}} Q_{j}^{\Omega}, Q_{i}^{\Omega}\right\rangle_{\mathcal{Q}^{*} \times \mathcal{Q}} & \text { otherwise } .\end{cases}
\end{aligned}
$$

At a specific node, the Dirichlet condition can be applied by using the Kronecker delta operator $\delta_{i j}$ as it can be seen in the first row of both matrix-specifications. Of course, the corresponding function value must still be specified. As it will be shown later, the function value can then be set either on the right hand side of the overall system of equation or within other matrices in an appropriate way. The matrices that link both velocity components can be assembled by using the formulas

$$
\begin{aligned}
& \mathrm{K}_{i j}^{v_{1} v_{2}}= \begin{cases}0 & \text { if } i \in \mathrm{B}_{(1)}^{v_{1}} \\
\left\langle K^{v_{1} v_{2}} Q_{j}^{\Omega}, Q_{i}^{\Omega}\right\rangle_{\mathcal{Q}^{*} \times \mathcal{Q}} & \text { otherwise, }\end{cases} \\
& \mathrm{K}_{i j}^{v_{2} v_{1}}= \begin{cases}0 & \text { if } i \in \mathrm{B}_{(1)}^{v_{2}} \\
\left\langle K^{v_{2} v_{1}} Q_{j}^{\Omega}, Q_{i}^{\Omega}\right\rangle_{\mathcal{Q}^{*} \times \mathcal{Q}} & \text { otherwise } .\end{cases}
\end{aligned}
$$

In order to preserve the Dirichlet condition, the respective rows (that are associated with a node that belongs to the Dirichlet boundary) have to vanish. For the same reasons, the configuration of the matrices that implement the interaction with the pressure field is given by

$$
\begin{aligned}
& \mathrm{K}_{i j}^{v_{1} p}= \begin{cases}0 & \text { if } i \in \mathrm{B}_{(1)}^{v_{1}} \\
\left\langle K^{v_{1} p} L_{j}^{\Omega}, Q_{i}^{\Omega}\right\rangle_{\mathcal{Q}^{*} \times \mathcal{Q}} & \text { otherwise }\end{cases} \\
& \mathrm{K}_{i j}^{v_{2} p}= \begin{cases}0 & \text { if } i \in \mathrm{B}_{(1)}^{v_{2}} \\
\left\langle K^{v_{2} p} L_{j}^{\Omega}, Q_{i}^{\Omega}\right\rangle_{\mathcal{Q}^{*} \times \mathcal{Q}} & \text { otherwise }\end{cases}
\end{aligned}
$$


The principle of the conversation of momentum states that the force that is applied on a collection of fluid particles and that can be represented by the use of the stress tensor is equal to the rate of change of linear momentum (cf. [36]). In the case of the first order system, the time-rate of change of the momentum can be discretized with the help of the matrices $\mathbf{H}^{v_{1} v_{1}}$ and $\mathbf{H}^{v_{2} v_{2}}$. Its components can be evaluated to

$$
\begin{aligned}
& \mathrm{H}_{i j}^{v_{1} v_{1}}= \begin{cases}0 & \text { if } i \in \mathrm{B}_{(1)}^{v_{1}} \\
\left\langle H^{v_{1} v_{1}} Q_{j}^{\Omega}, Q_{i}^{\Omega}\right\rangle_{\mathcal{Q}^{*} \times \mathcal{Q}} & \text { otherwise },\end{cases} \\
& \mathrm{H}_{i j}^{v_{2} v_{2}}= \begin{cases}0 & \text { if } i \in \mathrm{B}_{(1)}^{v_{2}} \\
\left\langle H^{v_{2} v_{2}} Q_{j}^{\Omega}, Q_{i}^{\Omega}\right\rangle_{\mathcal{Q}^{*} \times \mathcal{Q}} & \text { otherwise } .\end{cases}
\end{aligned}
$$

All terms of the momentum equations are taken into account by the matrices introduced above. But, the function values at the Dirichlet nodes still need to be specified. At the oval window, the first velocity component is given by the time-derivative of the predefined displacement of the stapes. Since the longitudinal velocity component vanishes at all other nodes of $\mathrm{B}_{(1)}^{v_{1}}$ and there is no other load that has to be considered, the right hand side vector of the velocity $v_{1}$ is given by

$$
\mathrm{F}_{i}^{v_{1}}= \begin{cases}\frac{\partial \xi_{\mathrm{ow}}(\boldsymbol{x})}{\partial t} & \text { if } i \in \mathrm{I}^{\mathcal{Q}\left(M^{\Gamma \mathrm{ow}}\right)} \\ 0 & \text { otherwise }\end{cases}
$$

Similarly, the second component of the velocity is affected by the vertical displacement of the basilar membrane. But in contrast to the oval window, the displacement of the cochlear partition is not predefined and it depends on the mutual interaction with the fluid. Therefore, the respective function values at the basilar membrane need to be determined in dependence of the nodal and unknown vector $\dot{\hat{\boldsymbol{\xi}}}$ that specifies the first time-derivative of the basilar membrane displacement (see below). In doing this, the velocity at the node $n_{i}$ that belongs to either the upper or lower partition boundary can be equated with the component $\psi_{b+}{ }^{-1}(i)$ of the velocity vector $\dot{\hat{\boldsymbol{\xi}}}$ in the case of the upper boundary, or with the component $\psi_{b-}{ }^{-1}(i)$ in the case of the lower boundary respectively. This relation can be realized by using a matrix that is given by

$$
\mathrm{H}_{i j}^{v_{2} \xi}= \begin{cases}-\delta_{i \psi_{b+}(j)} & \text { if } i \in \mathrm{B}^{\mathcal{Q}\left(M^{\Gamma_{\mathrm{bm}}+}\right)} \\ -\delta_{i \psi_{b-}(j)} & \text { if } i \in \mathrm{B}^{\mathcal{Q}\left(M^{\Gamma_{\mathrm{bm}-}}\right)} \\ 0 & \text { otherwise }\end{cases}
$$

Since the second velocity component vanishes at the other boundaries and since no external load exists, the right hand vector $\mathbf{F}^{v_{2}}$ is a null vector. Now, all these matrices can be used to transform the variational formulation of the balance-of-momentum equation into its semi-discretized formulation, which can be written as

$$
\begin{aligned}
& \mathbf{H}^{v_{1} v_{1}} \dot{\hat{\mathbf{v}}}_{1}^{(1)}+\mathbf{K}^{v_{1} v_{1}} \hat{\mathbf{v}}_{1}^{(1)}+\mathbf{K}^{v_{1} v_{2}} \hat{\mathbf{v}}_{2}^{(1)}+\mathbf{K}^{v_{1} p} \hat{\mathbf{p}}^{(1)}=\mathbf{F}^{v_{1}}, \\
& \mathbf{H}^{v_{2} v_{2}} \dot{\hat{\mathbf{v}}}_{2}^{(1)}+\mathbf{K}^{v_{2} v_{1}} \hat{\mathbf{v}}_{1}^{(1)}+\mathbf{K}^{v_{2} v_{2}} \hat{\mathbf{v}}_{2}^{(1)}+\mathbf{K}^{v_{2} p} \hat{\mathbf{p}}^{(1)}+\mathbf{H}^{v_{2} \xi} \dot{\hat{\boldsymbol{\xi}}}=\mathbf{0} .
\end{aligned}
$$


Similar to the principle of conservation of momentum, also the balance of mass equation consists of two different parts. The first part describes the rate of change of the mass with respect to the time. Its first order term can be transformed into a discretized formulation by establishing the matrix $\mathbf{H}^{p p}$. Since no boundary conditions are specified with regard to the pressure field, the entries of this matrix can be evaluated to

$$
\mathrm{H}_{i j}^{p p}=\left\langle H^{p p} L_{j}^{\Omega}, L_{i}^{\Omega}\right\rangle_{\mathcal{L}^{*} \times \mathcal{L}} .
$$

The second part is associated with the supply of mass. It constitutes a mixed term, due to the link to the velocity field. By applying the common discretization method on the first order problem, the matrices $\mathbf{K}^{p v_{1}}$ and $\mathbf{K}^{p v_{2}}$ can be deduced. Its components can be calculated to

$$
\begin{aligned}
& \mathrm{K}_{i j}^{p v_{1}}=\left\langle K^{p v_{1}} Q_{j}^{\Omega}, L_{i}^{\Omega}\right\rangle_{\mathcal{L}^{*} \times \mathcal{L}}, \\
& \mathrm{K}_{i j}^{p v_{2}}=\left\langle K^{p v_{2}} Q_{j}^{\Omega}, L_{i}^{\Omega}\right\rangle_{\mathcal{L}^{*} \times \mathcal{L}} .
\end{aligned}
$$

All in all, the discretized first order equation that origins from the mass conservation can now be written in terms of the former matrices as

$$
\mathbf{H}^{p p} \dot{\hat{\mathbf{p}}}^{(1)}+\mathbf{K}^{p v_{1}} \hat{\mathbf{v}}_{1}^{(1)}+\mathbf{K}^{p v_{2}} \hat{\mathbf{v}}_{2}^{(1)}=0 .
$$

The equations 3.68, 3.69 and 3.73 constitutes the semi-discretized version of the first order fluidic subproblem.

Basilar Membrane The displacement of the basilar membrane is mainly driven by the difference between the pressure above and below the cochlear partition. Since the mesh of the basilar membrane is, moreover, identical to parts of the boundary that belongs to the fluid domain, it is advisable to use the same type of basis functions for the evaluation of the dynamics of the basilar membrane as used for the pressure field. Therefore, the function space that represents the displacement of the cochlear partition should be discretized by using the space $\mathcal{L}\left(M^{\Gamma_{\mathrm{bm}}}\right)$ (cf. equation 3.45) .

Otherwise, the velocity of adjacent fluid-particles depends on the displacement of the basilar membrane. Therefore, it would be useful if the displacement of the basilar membrane is also available at nodes that are exclusively used by the quadratic basis functions.

In order to meet both requirements a combined approach is used. Although the principle computation is based on linear basis functions, the displacement at the additional quadratic nodes are also interpolated on the basis of values at the adjacent linear nodes. In doing this, the additional nodes (that are used in order to constitute the quadratic basis functions but that do not belong to the linear basis functions) can be described by the index family

$$
\mathrm{I}^{\mathcal{Q} \backslash \mathcal{L}}:=\mathrm{I}^{\mathcal{Q}\left(M^{\Gamma \mathrm{bm}}\right)} \backslash\left\{\varphi(i) \mid i \in \mathrm{I}^{\mathcal{L}\left(M^{\Gamma \mathrm{bm}}\right)}\right\} .
$$

A simple linear interpolation technique that can be used for determining the displacement at the nodes that correspond to the index family 3.74 needs to have access to the respective 
values at its direct neighbors. Therefore, the indices of the neighbors of the node that is associated with index $i \in \mathrm{I}^{\mathcal{Q} \backslash \mathcal{L}}$ are given by the set

$$
\mathrm{I}^{N}(i):=\left\{j \in \mathrm{I}^{\mathcal{L}\left(M^{\Gamma_{\mathrm{bm}}}\right)} \mid j \text { belongs to the same simplex as } i\right\} .
$$

In summary, the discretization process is realized by using matrices, whose structure is based on the function space $\mathcal{Q}\left(M^{\Gamma \mathrm{bm}}\right)$ (cf. equation 3.46$)$. But in contrast to the matrices above, its entries are assembled by using dual pairs that are defined on the function space $\mathcal{L}\left(M^{\Gamma_{\mathrm{bm}}}\right)$ (cf. equation 3.45). Then, the stiffness matrix is given by

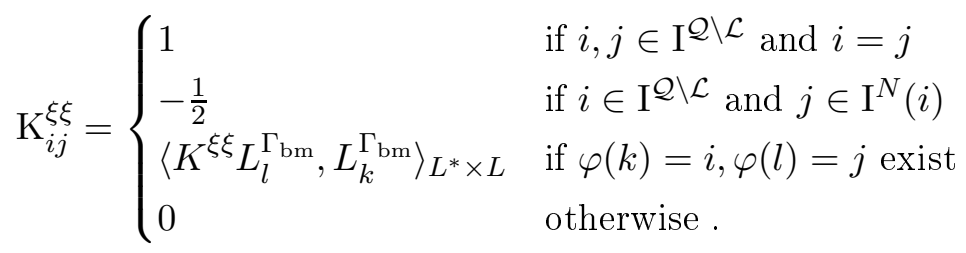

The first two rows realize the interpolation of the displacement by evaluating the average value of the two neighbouring displacement values. The third row is associated with the longitudinal varying stiffness of the basilar membrane.

Of course, it would also be possible to directly implement the linear interpolation by an adaption of the entries of the matrix $\mathbf{K}^{v_{2} v_{2}}$. Furthermore, other implementation techniques might also be implemented like cubic $C^{2}$ splines. But a possible higher accuracy seems to be disproportionate to the resultant computational effort.

As described in chapter 2.3.2, the damping of the oscillatory model consists of two different damping components. Both, the longitudinal shearing resistance as well as the intrinsic viscosity of the cochlear partition at a specific point are considered by the matrix

$$
\mathrm{H}_{i j}^{\xi \xi}= \begin{cases}\left\langle H^{\xi \xi} L_{l}^{\Gamma_{\mathrm{bm}}}, L_{k}^{\Gamma_{\mathrm{bm}}}\right\rangle_{L^{*} \times L} & \text { if } \varphi(k)=i, \varphi(l)=j \text { exist } \\ 0 & \text { otherwise } .\end{cases}
$$

The discretized counterpart of the term that describes the inertial reaction of the basilar membrane is given by

$$
\mathrm{M}_{i j}^{\xi \xi}= \begin{cases}\left\langle M^{\xi \xi} L_{l}^{\Gamma_{\mathrm{bm}}}, L_{k}^{\Gamma_{\mathrm{bm}}}\right\rangle_{L^{*} \times L} & \text { if } \varphi(k)=i, \varphi(l)=j \text { exist } \\ 0 & \text { otherwise } .\end{cases}
$$

Now, the dynamics of the passive basilar membrane are fully described by the matrices $\mathbf{K}^{\xi \xi}, \mathbf{H}^{\xi \xi}$ and $\mathbf{M}^{\xi \xi}$. As mentioned above, the cochlear system is stimulated by the fluidpressure above and below the partition. This external pressure-load can be represented by a matrix, whose components can be evaluated to

$$
\mathrm{K}_{i j}^{\xi p}= \begin{cases}\left\langle K^{\xi p} L_{l}^{\Omega}, L_{k}^{\Gamma_{\mathrm{bm}}}\right\rangle_{L^{*} \times L} & \text { if } \varphi(k)=i, \varphi(l)=j \text { exist } \\ 0 & \text { otherwise } .\end{cases}
$$


Furthermore, an additional force may be applied by the outer hair cell motility. Since this external load is assumed to be proportional to small deflections of the hair bundles, the discretization process yields the matrix

$$
\mathrm{K}_{i j}^{\xi \zeta}= \begin{cases}\left\langle K^{\xi \zeta} L_{l}^{\Gamma_{\mathrm{bm}}}, L_{k}^{\Gamma_{\mathrm{bm}}}\right\rangle_{L^{*} \times L} & \text { if } \varphi(k)=i, \varphi(l)=j \text { exist } \\ 0 & \text { otherwise } .\end{cases}
$$

Now, a semi-discretized system that simulates the displacement of the cochlear partition can be established by using the matrices introduced above. In doing this, the variational formulation 3.11 can be transformed into the ordinary differential equation

$$
\mathbf{M}^{\xi \xi} \ddot{\hat{\boldsymbol{\xi}}}+\mathbf{H}^{\xi \xi} \dot{\hat{\boldsymbol{\xi}}}+\mathbf{K}^{\xi \xi} \hat{\boldsymbol{\xi}}+\mathbf{K}^{\xi p} \hat{\mathbf{p}}^{(1)}+\mathbf{K}^{\xi \zeta} \hat{\boldsymbol{\zeta}}=\mathbf{0} .
$$

Outer Hair Cell Motility As described in chapter 2.3.3, the deflection of the stereocilia is modeled as an additional oscillator. Therefore, the associated differential equation consists of a stiffness-, a damping- and a mass-term. These physical characteristics are reflected in the matrices $\mathbf{K}^{\zeta \zeta}, \mathbf{H}^{\zeta \zeta}$ and $\mathbf{M}^{\zeta \zeta}$. The matrices can be evaluated on the basis of the variational formulation 3.14. According to the basis functions of the basilar membrane motion, the deflections of the hair bundles are also approximated by piecewise linear functions, because the displacement of the cochlear partition is a direct cause of the deflection. The components of the matrices can be assembled by using the following formulas:

$$
\begin{aligned}
\mathrm{K}_{i j}^{\zeta \zeta} & =\left\langle K^{\zeta \zeta} L_{j}^{\Gamma_{\mathrm{bm}}}, L_{i}^{\Gamma_{\mathrm{bm}}}\right\rangle_{L^{*} \times L}, \\
\mathrm{H}_{i j}^{\zeta \zeta} & =\left\langle H^{\zeta \zeta} L_{j}^{\Gamma_{\mathrm{bm}}}, L_{i}^{\Gamma_{\mathrm{bm}}}\right\rangle_{L^{*} \times L}, \\
\mathrm{M}_{i j}^{\zeta \zeta} & =\left\langle M^{\zeta \zeta} L_{j}^{\Gamma_{\mathrm{bm}}}, L_{i}^{\Gamma_{\mathrm{bm}}}\right\rangle_{L^{*} \times L} .
\end{aligned}
$$

The coupling to the basilar membrane displacement is realized by the matrix $\mathbf{M}^{\zeta \xi}$ whose components can be calculated to

$$
\mathrm{M}_{i j}^{\zeta \xi}=\left\langle M^{\zeta \xi} L_{j}^{\Gamma_{\mathrm{bm}}}, L_{i}^{\Gamma_{\mathrm{bm}}}\right\rangle_{L^{*} \times L} .
$$

By the use of these matrices, the differential equation that describes the deflection of the hair bundles can be transformed into its semi-discretized formulation, which is given by

$$
\mathbf{M}^{\zeta \zeta} \ddot{\hat{\boldsymbol{\zeta}}}+\mathbf{H}^{\zeta \zeta \dot{\hat{\boldsymbol{\zeta}}}}+\mathbf{K}^{\zeta \zeta} \hat{\boldsymbol{\zeta}}+\mathbf{M}^{\zeta \xi \ddot{\boldsymbol{\xi}}}=\mathbf{0} \text {. }
$$

\subsubsection{Multiphysical Coupling}

The first order system can be regarded as a multiphysical problem, since the system can only be realistically described by the simultaneous consideration of multiple physical phenomena. The multiphysical coupling of the acoustic subproblem can be summarized by the following interactions: 
- The displacement of the basilar membrane is directly affected by the fluid pressure above and below the cochlear partition.

- The fluid velocity at the basilar membrane above and below the cochlear partition depends on the displacement of the basilar membrane.

- The outer hair cell force appears as an additional load which acts on the basilar membrane.

- The outer hair cell force is mainly influenced by the displacement of the basilar membrane as a consequence of the relative motions of the reticular lamina with respect to the tectorial membrane.

Therefore, the dynamics of the fluid, the basilar membrane and the tectorial membrane constitute three different physical processes that have to be simultaneously taken into account by the overall simulation process, since the individual processes have a mutual influence on each other.

In contrast to the interaction of these three components, the coupling between the fluid and the round window is realized through an internal boundary condition with respect to the longitudinal velocity field (cf. chapter 2.2.3). Due to this specific kind of implementation, the interaction between the round window and the fluid can therefore not be considered as a multiphysical phenomenon. Also the fluid itself may be considered as a coupled system, since the pressure and the velocity variables are coupled by the conservation principles and constitutive relations as described above. Since the fluid dynamics are modeled as a whole by the interplay between the pressure and the velocity field, this kind of interaction is referred to as a mixed formulation rather than a coupled phenomenon.

Multiphysical interactions between two physical processes are often categorized by the degree to which they are coupled. In literature, a distinction is often made between a strong and weak coupling. But neither a formal definition nor a quantification has been established up to now in order to objectify the degree of coupling.

In principle, two different approaches for the numerical implementation of a multiphysical problem can be distinguished. The first approach for simulating multiple processes involves the consideration of the coupled processes as one monolithic system of equations. It is a straightforward method which naturally takes the coupling into account even if the mutual interactions have significant influences on each individual process. The major drawback of this procedure is that the resultant system of equations may become quite large and its associated matrix is potentially badly conditioned. In contrast, the second approach is based on separate sub-routines that are specialized in the numerical computation of the different physical phenomena that are involved. In this case, the coupling is realized by the transfer of relevant data, which can be taken into account by specific boundary conditions, load terms, geometric shape or constitutive relations. If the coupling is not unidirectional, the results of the processes may have a mutual influence to such an extent that the computation of each sub-system has to be repeated (under consideration of the updated data) by an iterative sub-process in order to achieve accurate results. Whether 
such an iterative process is really necessary depends mainly on the degree of influence on each other. In many applications, it seems to be sufficient to transfer the coupling-relevant data from one process to the other just one time per time-step or even only an occasional update every few times steps is enough to adequately represent the multiphysical system. An advantage of such a partitioned method lies in a more effective computation of each individual sub-process since each sub-routine can be numerically optimized and in most cases the resultant sub-systems of equations are significantly smaller and better conditioned than the overall system. Furthermore, the modular design of the implementation, which is characterized by the application of different modules for each individual physical phenomena, facilitates the reuse of the software with respect to other computations. (cf. [36, 15])

In this work, the multiphysical problem is realized by a monolithic system of equations. It can be assumed that a partitioned realization of the coupling involves a substantial computational effort that is probably needed by the application of the sub-iterations (as mentioned above) due to the strong interdependence between the fluid, the basilar membrane and the outer hair cell motility. Therefore, it can not be expected that the partitioned implementation has significant advantages with respect to the computational effort over a monolithic realization. Furthermore, the modular design is accompanied with a substantial increase of the code complexity, due to the additional implementation of adequate interfaces, data transfer protocols, finite element interpolations and the monitoring of the sub-iteration processes. Moreover, the cochlear system is a highly specific problem (in particular with regard to the basilar membrane), so that the resultant code can not be easily adopted by other applications for the simulation of acoustically driven flows within a fluid-structure coupled system. Nevertheless, also the monolithic scheme is accompanied by increased requirements on the software architecture.

According to the equations 3.68, 3.69, 3.73, 3.81 and 3.86, the fully coupled monolithic system of the first order acoustic subproblem can be represented as

$$
\mathbf{M u ̈}+\mathbf{H} \dot{\mathbf{u}}+\mathbf{K u}=\mathbf{F}
$$

which constitutes a system of second order linear ordinary differential equations. The nodal vector $\mathbf{u}$ combines the individual nodal vectors of the fluid velocity, fluid pressure, basilar membrane displacement and hair bundle deflection and this overall vector can be written as

$$
\mathbf{u}=\left[\begin{array}{lllll}
\hat{\mathbf{v}}_{1}^{(1)} & \hat{\mathbf{v}}_{2}^{(1)} & \hat{\mathbf{p}}^{(1)} & \hat{\boldsymbol{\xi}} & \hat{\boldsymbol{\zeta}}
\end{array}\right]^{T}
$$

Due to the similarity of equation 3.87 to equations of motions where the vector $\mathbf{u}$ is usually identified with the displacement of the motion, the matrices $\mathbf{M}, \mathbf{H}$ and $\mathbf{K}$ are often referred to as the mass matrix, the damping matrix and the stiffness matrix. From a physical point of view it must be noted that these terms are not precise with respect to the ordinary differential equation presented here, since the nodal vector $\mathbf{u}$ consists not only of displacement components but also of velocity components. By using the sub matrices of the individual equations as introduced in section 3.4.1, the stiffness matrix can be written 
as

$$
\mathbf{K}=\left[\begin{array}{ccccc}
\mathbf{K}^{v_{1} v_{1}} & \mathbf{K}^{v_{1} v_{2}} & \mathbf{K}^{v_{1} p} & \mathbf{0} & \mathbf{0} \\
\mathbf{K}^{v_{2} v_{1}} & \mathbf{K}^{v_{2} v_{2}} & \mathbf{K}^{v_{2} p} & \mathbf{0} & \mathbf{0} \\
\mathbf{K}^{p v_{1}} & \mathbf{K}^{p v_{2}} & \mathbf{0} & \mathbf{0} & \mathbf{0} \\
\mathbf{0} & \mathbf{0} & \mathbf{K}^{\xi p} & \mathbf{K}^{\xi \xi} & \mathbf{K}^{\xi \zeta} \\
\mathbf{0} & \mathbf{0} & \mathbf{0} & \mathbf{0} & \mathbf{K}^{\zeta \zeta}
\end{array}\right] .
$$

The discretized damping matrix of the first order subproblem can be represented as

$$
\mathbf{H}=\left[\begin{array}{ccccc}
\mathbf{H}^{v_{1} v_{1}} & \mathbf{0} & \mathbf{0} & \mathbf{0} & \mathbf{0} \\
\mathbf{0} & \mathbf{H}^{v_{2} v_{2}} & \mathbf{0} & \mathbf{H}^{v_{2} \xi} & \mathbf{0} \\
\mathbf{0} & \mathbf{0} & \mathbf{H}^{p p} & \mathbf{0} & \mathbf{0} \\
\mathbf{0} & \mathbf{0} & \mathbf{0} & \mathbf{H}^{\xi \xi} & \mathbf{0} \\
\mathbf{0} & \mathbf{0} & \mathbf{0} & \mathbf{0} & \mathbf{H}^{\zeta \zeta}
\end{array}\right]
$$

Finally, the overall mass matrix can be determined to

$$
\mathbf{M}=\left[\begin{array}{ccccc}
\mathbf{0} & \mathbf{0} & \mathbf{0} & \mathbf{0} & \mathbf{0} \\
\mathbf{0} & \mathbf{0} & \mathbf{0} & \mathbf{0} & \mathbf{0} \\
\mathbf{0} & \mathbf{0} & \mathbf{0} & \mathbf{0} & \mathbf{0} \\
\mathbf{0} & \mathbf{0} & \mathbf{0} & \mathbf{M}^{\xi \xi} & \mathbf{0} \\
\mathbf{0} & \mathbf{0} & \mathbf{0} & \mathbf{M}^{\zeta \xi} & \mathbf{M}^{\zeta \zeta}
\end{array}\right]
$$

By the monolithic matrix representation, the interdependencies (or in other words the coupling) between different field variables are easily recognizable by the sub-matrices that are not located on the main diagonal.

The right hand side of the first order semi-discretized formulation 3.87 consists only of entries belonging to the longitudinal velocity components that are associated with the displacement of the oval window. Therefore, the load vector can be written as

$$
\mathbf{F}=\left[\begin{array}{lllll}
\mathbf{F}^{v_{1}} & \mathbf{0} & \mathbf{0} & \mathbf{0} & \mathbf{0}
\end{array}\right]^{T} .
$$

\subsubsection{The Second Order System}

Acoustic Streaming Subproblem As shown in chapter 2.4 the mean motion of the secondary flow is characterized on the one hand by the second order supply-terms of the mass and momentum and on the other hand by the virtual source-distributions of mass and momentum. The supply of linear momentum in terms of the second order mean variables $\boldsymbol{v}^{(d c)}$ and $p^{(d c)}$ can be represented by the matrices $\mathbf{G}^{v_{1} v_{1}}, \mathbf{G}^{v_{2} v_{2}}, \mathbf{G}^{v_{1} v_{2}}, \mathbf{G}^{v_{2} v_{1}}$, 
$\mathbf{G}^{v_{1} p}$ and $\mathbf{G}^{v_{2} p}$. In order to take also the boundary conditions into account, the indices of the boundary nodes are combined into the set

$$
\mathrm{B}_{(d c)}^{v}=\mathrm{B}^{\mathcal{Q}\left(M^{\Gamma_{\mathrm{ow}}}\right)} \cup \mathrm{B}^{\mathcal{Q}\left(M^{\Gamma_{\mathrm{rw}}}\right)} \cup \mathrm{B}^{\mathcal{Q}\left(M^{\Gamma_{\mathrm{bm}}+}\right)} \cup \mathrm{B}^{\mathcal{Q}\left(M^{\Gamma_{\mathrm{bm}}-}\right)} \cup \mathrm{B}^{\mathcal{Q}\left(M^{\Gamma_{\mathrm{r}}}\right)} .
$$

Using this index family, the first two matrices can be assembled by using the relations

$$
\begin{aligned}
\mathrm{G}_{i j}^{v_{1} v_{1}} & = \begin{cases}\delta_{i j} & \text { if } i \in \mathrm{B}_{(d c)}^{v} \\
\left\langle G^{v_{1} v_{1}} Q_{j}^{\Omega}, Q_{i}^{\Omega}\right\rangle_{\mathcal{Q}^{*} \times \mathcal{Q}} & \text { otherwise },\end{cases} \\
\mathrm{G}_{i j}^{v_{2} v_{2}} & = \begin{cases}\delta_{i j} & \text { if } i \in \mathrm{B}_{(d c)}^{v} \\
\left\langle G^{v_{2} v_{2}} Q_{j}^{\Omega}, Q_{i}^{\Omega}\right\rangle_{\mathcal{Q}^{*} \times \mathcal{Q}} & \text { otherwise } .\end{cases}
\end{aligned}
$$

As in the case of the first order subproblem, the Kronecker delta symbol allows the specification of the velocity components at the boundaries by the values of the right hand side. By taking into account that all other entries at those rows that are associated with a boundary node must vanish, the matrices that connect both velocity components are given by

$$
\begin{aligned}
& \mathrm{G}_{i j}^{v_{1} v_{2}}= \begin{cases}0 & \text { if } i \in \mathrm{B}_{(d c)}^{v} \\
\left\langle G^{v_{1} v_{2}} Q_{j}^{\Omega}, Q_{i}^{\Omega}\right\rangle_{\mathcal{Q}^{*} \times \mathcal{Q}} & \text { otherwise },\end{cases} \\
& \mathrm{G}_{i j}^{v_{2} v_{1}}= \begin{cases}0 & \text { if } i \in \mathrm{B}_{(d c)}^{v} \\
\left\langle G^{v_{2} v_{1}} Q_{j}^{\Omega}, Q_{i}^{\Omega}\right\rangle_{\mathcal{Q}^{*} \times \mathcal{Q}} & \text { otherwise } .\end{cases}
\end{aligned}
$$

Due to the LBB-condition (cf. section 3.4.1) the maximal degree of the polynomial basis functions that represent the pressure variable must be one order less than the basis functions of the velocity field. Therefore, the coupling to the pressure variable can be implemented by the matrices

$$
\begin{aligned}
& \mathrm{G}_{i j}^{v_{1} p}= \begin{cases}0 & \text { if } i \in \mathrm{B}_{(d c)}^{v} \\
\left\langle G^{v_{1} p} L_{j}^{\Omega}, Q_{i}^{\Omega}\right\rangle_{\mathcal{Q}^{*} \times \mathcal{Q}} & \text { otherwise },\end{cases} \\
& \mathrm{G}_{i j}^{v_{2} p}= \begin{cases}0 & \text { if } i \in \mathrm{B}_{(d c)}^{v} \\
\left\langle G^{v_{2} p} L_{j}^{\Omega}, Q_{i}^{\Omega}\right\rangle_{\mathcal{Q}^{*} \times \mathcal{Q}} & \text { otherwise } .\end{cases}
\end{aligned}
$$

The force source distribution results in the load vector $\mathbf{S}^{v_{1}}$ and $\mathbf{S}^{v_{2}}$, whose components can be calculated to

$$
\begin{aligned}
& \mathrm{S}_{i}^{v_{1}}= \begin{cases}\tilde{f}_{1}(\boldsymbol{x}) & \text { if } i \in \mathrm{B}_{(d c)}^{v} \\
\left\langle S^{v_{1}}, Q_{i}^{\Omega}\right\rangle_{\mathcal{Q}^{*} \times \mathcal{Q}} & \text { otherwise, }\end{cases} \\
& \mathrm{S}_{i}^{v_{2}}= \begin{cases}\tilde{f}_{2}(\boldsymbol{x}) & \text { if } i \in \mathrm{B}_{(d c)}^{v} \\
\left\langle S^{v_{2}}, Q_{i}^{\Omega}\right\rangle_{\mathcal{Q}^{*} \times \mathcal{Q}} & \text { otherwise } .\end{cases}
\end{aligned}
$$

According to equation 2.49, the first row of these relations specifies the values at the Dirichlet boundaries, whereby the coordinate $\boldsymbol{x}$ indicates the respective location of the 
actual node. The second row is associated with the force distribution within the bulk. Now, the semi-discretized system that represents the supply of force in combination with the force source distribution can be written as

$$
\begin{aligned}
& \mathbf{G}^{v_{1} v_{1}} \hat{\mathbf{v}}_{1}^{(d c)}+\mathbf{G}^{v_{1} v_{2}} \hat{\mathbf{v}}_{2}^{(d c)}+\mathbf{G}^{v_{1} p} \hat{\mathbf{p}}^{(d c)}=\mathbf{S}^{v_{1}}, \\
& \mathbf{G}^{v_{2} v_{1}} \hat{\mathbf{v}}_{1}^{(d c)}+\mathbf{G}^{v_{2} v_{2}} \hat{\mathbf{v}}_{2}^{(d c)}+\mathbf{G}^{v_{2} p} \hat{\mathbf{p}}^{(d c)}=\mathbf{S}^{v_{2}} .
\end{aligned}
$$

The supply of mass with respect to the second order subproblem is associated with the matrices $\mathbf{G}^{p v_{1}}$ and $\mathbf{G}^{p v_{2}}$. Similar to above, the set

$$
\mathrm{B}_{(d c)}^{p}=\mathrm{B}^{\mathcal{L}\left(M^{\Gamma_{\mathrm{ow}}}\right)} \cup \mathrm{B}^{\mathcal{L}\left(M^{\Gamma_{\mathrm{rw}}}\right)} \cup \mathrm{B}^{\mathcal{L}\left(M^{\Gamma_{\mathrm{bm}}+}\right)} \cup \mathrm{B}^{\mathcal{L}\left(M^{\Gamma_{\mathrm{bm}}-}\right)} \cup \mathrm{B}^{\mathcal{L}\left(M^{\Gamma_{\mathrm{r}}}\right)}
$$

specifies the indices that are associated with the boundary nodes in order to establish the Dirichlet boundary condition. With the help of this set of indices, the entries of the matrices can be calculated to

$$
\begin{aligned}
& \mathrm{G}_{i j}^{p v_{1}}= \begin{cases}0 & \text { if } i \in \mathrm{B}_{(d c)}^{p} \\
\left\langle G^{p v_{1}} Q_{j}^{\Omega}, L_{i}^{\Omega}\right\rangle_{\mathcal{L}^{*} \times \mathcal{L}} & \text { otherwise },\end{cases} \\
& \mathrm{G}_{i j}^{p v_{2}}= \begin{cases}0 & \text { if } i \in \mathrm{B}_{(d c)}^{p} \\
\left\langle G^{p v_{2}} Q_{j}^{\Omega}, L_{i}^{\Omega}\right\rangle_{\mathcal{L}^{*} \times \mathcal{L}} & \text { otherwise } .\end{cases}
\end{aligned}
$$

In order to implement the Dirichlet boundary condition, the respective entries on the main diagonal of the matrix $\mathbf{G}^{p p}$ must be set to 1 . In terms of mathematical formulation, this matrix is given by

$$
\mathrm{G}_{i j}^{p p}= \begin{cases}\delta_{i j} & \text { if } i \in \mathrm{B}_{(d c)}^{p} \\ 0 & \text { otherwise } .\end{cases}
$$

Similar to the force source distribution, the mass source distribution (cf. equation 2.47) can be implemented as a right hand vector $\mathbf{S}^{p}$. Thereby, a distinction between the internal distribution and the boundary values must be drawn. Then, its components are given by

$$
\mathrm{S}_{i}^{p}= \begin{cases}\tilde{m}(\boldsymbol{x}) & \text { if } i \in \mathrm{B}_{(d c)}^{p} \\ \left\langle S^{p}, L_{i}^{\Omega}\right\rangle_{\mathcal{L}^{*} \times \mathcal{L}} & \text { otherwise }\end{cases}
$$

Finally, this load vector in combination with the matrices, introduced above, can be brought together in the system of equations

$$
\mathbf{G}^{p p} \hat{\mathbf{p}}^{(d c)}+\mathbf{G}^{p v_{1}} \hat{\mathbf{v}}_{1}^{(d c)}+\mathbf{G}^{p v_{2}} \hat{\mathbf{v}}_{2}^{(d c)}=\mathbf{S}^{p}
$$

in order to obtain the semi-discretized formulation of the second order mass conservation equation. 
The overall mean second order system, which yields a first order approximation of the acoustically driven flows, can be established by combining equations $3.102,3.103$ and 3.109 , This system can be written as

$$
\left[\begin{array}{ccc}
\mathbf{G}^{v_{1} v_{1}} & \mathbf{G}^{v_{1} v_{2}} & \mathbf{G}^{v_{1} p} \\
\mathbf{G}^{v_{2} v_{1}} & \mathbf{G}^{v_{2} v_{2}} & \mathbf{G}^{v_{2} p} \\
\mathbf{G}^{p v_{1}} & \mathbf{G}^{p v_{2}} & \mathbf{G}^{p p}
\end{array}\right]\left[\begin{array}{c}
\hat{\mathbf{v}}_{1}^{(d c)} \\
\hat{\mathbf{v}}_{2}^{(d c)} \\
\hat{\mathbf{p}}^{(d c)}
\end{array}\right]=\left[\begin{array}{c}
\mathbf{S}^{v_{1}} \\
\mathbf{S}^{v_{2}} \\
\mathbf{S}^{p}
\end{array}\right]
$$

\subsubsection{Assemblage}

This chapter is concerned with the assemblage of the individual sub-matrices that are needed to establish the time-variant first order system 3.87 as well as the stationary mean second order system 3.110. In principle, the assemblage strategies are based on the toolbox Alberta [42, 44]. Most of the entries of these matrices can be written as a dual pair $\left\langle L N_{i}, R_{j}\right\rangle$, where $N_{i}$ and $R_{i}$ denote (not necessarily the same class of) basis functions and $L$ represents an elliptic differential operator (cf. [23, 42]). Such an elliptic differential operator can be written as

$$
(L u)(\boldsymbol{x}):=-\operatorname{div}(\mathbf{A}(\boldsymbol{x}) \operatorname{grad} u(\boldsymbol{x}))+(\boldsymbol{b}(\boldsymbol{x}))^{T}(\operatorname{grad} u(\boldsymbol{x}))+c(\boldsymbol{x}) u(\boldsymbol{x})
$$

where the coefficient functions are given as

$$
\begin{aligned}
& \mathbf{A}: M \rightarrow \mathbb{R}^{d \times d}, \\
& \boldsymbol{b}: M \rightarrow \mathbb{R}^{d} \\
& c: M \rightarrow \mathbb{R} .
\end{aligned}
$$

The individual components of these coefficients should belong to the function space $L^{\infty}$. In this work, all differential operators can be represented by an elliptic differential operator as shown in equation 3.111. According to the derivation of the variational formulation (cf. chapter 3.21), the dual pair can be transformed into the form

$$
\begin{array}{r}
\left\langle L N_{i}, R_{j}\right\rangle=\int\left(\operatorname{grad} N_{i}\right)^{T} \mathbf{A}\left(\operatorname{grad} R_{j}\right) \mathrm{d} \boldsymbol{x}+ \\
\int N_{i} \boldsymbol{b}^{T}\left(\operatorname{grad} R_{j}\right) \mathrm{d} \boldsymbol{x}+\int c N_{i} R_{j} \mathrm{~d} \boldsymbol{x}
\end{array}
$$

Furthermore, the integral over the whole mesh can be splitted into a set of integrals that are specified on each individual simplex. Therefore, the dual pair can be updated according to the relation

$$
\begin{array}{r}
\left\langle L N_{i}, R_{j}\right\rangle=\sum_{S \in M}\left[\int_{S}\left(\operatorname{grad} N^{k_{S}(i)}\right)^{T} \mathbf{A}\left(\operatorname{grad} R^{k_{S}(j)}\right) \mathrm{d} \boldsymbol{x}+\right. \\
\left.\int_{S} N^{k_{S}(i)} \boldsymbol{b}^{T}\left(\operatorname{grad} R^{k_{S}(j)}\right) \mathrm{d} \boldsymbol{x}+\int_{S} c N^{k_{S}(i)} R^{k_{S}(j)} \mathrm{d} \boldsymbol{x}\right]
\end{array}
$$


where each index $i$ of the global basis functions have to be replaced by an index $l$ that is associated with the respective local basis function via the mapping $l=k_{S}(i)$. As described in chapter 3.3.2, the basis functions are specified with respect to the barycentric coordinates. Therefore, the basis functions can be replaced by their barycentric counterpart with the help of the mapping $\boldsymbol{\beta}(\boldsymbol{x})$. By the use of the chain rule, the gradient of the basis functions can be calculated to

$$
\begin{aligned}
\operatorname{grad} N^{k_{S}(i)}(\boldsymbol{x}) & =\operatorname{grad} N_{(\mathrm{bar})}^{k_{S}(i)}(\boldsymbol{\beta}(\boldsymbol{x})) \\
& =(D \boldsymbol{\beta}(\boldsymbol{x}))^{T}\left(\operatorname{grad}_{\beta} N_{(\mathrm{bar})}^{k_{S}(i)}(\boldsymbol{\beta}(\boldsymbol{x}))\right)
\end{aligned}
$$

In contrast to the basis functions, the numerical integration is performed over the normalized reference element. By transforming the integral from the world coordinates $\boldsymbol{x}$ to the normalized coordinates $\boldsymbol{\nu}$ and by using equation 3.115 , the first term of the right hand side of equation 3.114 can be evaluated to

$$
\begin{gathered}
\int_{S}\left(\operatorname{grad} N^{k(i)}(\boldsymbol{x})\right)^{T} \mathbf{A}(\boldsymbol{x})\left(\operatorname{grad} R^{l(j)}(\boldsymbol{x})\right) \mathrm{d} \boldsymbol{x}= \\
\int_{S}\left(\operatorname{grad}_{\beta} N_{(\text {bar })}^{k(i)}(\boldsymbol{\beta}(\boldsymbol{\nu}))\right)^{T}\left(D \boldsymbol{\beta}(\boldsymbol{x}(\boldsymbol{\nu})) \mathbf{A}(\boldsymbol{x})\left(D \boldsymbol{\beta}(\boldsymbol{x}(\boldsymbol{\nu}))^{T}\right)\right. \\
\left(\operatorname{grad}_{\beta} R_{(\text {bar })}^{k(i)}(\boldsymbol{\beta}(\boldsymbol{\nu}))\right)|\operatorname{det} D \boldsymbol{x}(\boldsymbol{\nu})| \mathrm{d} \nu
\end{gathered}
$$

The gradient of the basis functions can be precalculated for all simplices, since it depends only on the unvarying normalized and barycentric elements as well as the predefined basis functions itself. Therefore, only the term in the middle that consists of the coefficient function and the Jacobian-matrices of the barycentric coordinates with respect to the world coordinates as well as the determinant have to be evaluated for each individual element. The second term of the right hand side of equation 3.114 can also be expressed in terms of the normalized coordinates as

$$
\begin{gathered}
\int_{S} N^{k(i)}(\boldsymbol{x})(\boldsymbol{b}(\boldsymbol{x}))^{T}\left(\operatorname{grad} R^{l(j)}(\boldsymbol{x})\right) \mathrm{d} \boldsymbol{x}= \\
\int_{S}\left(N_{(\text {bar })}^{k(i)}(\boldsymbol{\beta}(\boldsymbol{\nu}))\right)\left(D \boldsymbol{\beta}(\boldsymbol{x}(\boldsymbol{\nu}) \boldsymbol{b}(\boldsymbol{x}))^{T}\right. \\
\left(\operatorname{grad}_{\beta} R_{(\text {bar })}^{k(i)}(\boldsymbol{\beta}(\boldsymbol{\nu}))\right)|\operatorname{det} D \boldsymbol{x}(\boldsymbol{\nu})| \mathrm{d} \nu
\end{gathered}
$$

In compliance with the first term, only the parts that depend on the world coordinates have to be evaluated at each individual simplex. The last term within the squared brackets of equation 3.114 can be calculated to

$$
\begin{aligned}
& \int_{S} c(\boldsymbol{x}) N^{k(i)}(\boldsymbol{x}) R^{l(j)}(\boldsymbol{x}) \mathrm{d} \boldsymbol{x}= \\
& \int_{S} c(\boldsymbol{x})\left(N_{(\text {bar })}^{k(i)}(\boldsymbol{\beta}(\boldsymbol{\nu}))\right)\left(R_{(\mathrm{bar})}^{k(i)}(\boldsymbol{\beta}(\boldsymbol{\nu}))\right)|\operatorname{det} D \boldsymbol{x}(\boldsymbol{\nu})| \mathrm{d} \nu
\end{aligned}
$$


Under the assumption that the coefficient functions are constant on each simplex of the mesh, a numerical quadrature method must only performed only once at the beginning of the assemblage process for all combinations of the local basis functions. All other terms are constant and can be separated from the integrals. A more detailed description of the assemblage process can be found in [42, 44].

As shown in chapter 2, some coefficient functions vary depending on the spatial coordinates. In these cases, a numerical quadrature approach has to be used in order to get numerical approximations of the respective integrals. According to [42, 23], the quadrature methods are based on the formula

$$
\int_{S} f(\boldsymbol{\nu}) \mathrm{d} \nu \approx \sum_{i=0}^{n} w_{i} f\left(\boldsymbol{\nu}_{i}\right)
$$

where $w_{i}$ denotes a weight that is associated with an $i$ th quadrature point $\boldsymbol{\nu}_{i}$.

\subsection{Temporal Discretization}

Up to now, the first order problem is only transformed into a semi-discretized formulation, since equation 3.87 further depends on the continuous time-variable $t$. In principle, it would also be conceivable to discretize the time in a similar way as the spatial variables by the use of finite elements. But, if the entire time frame is discretized by finite elements, the approach will result in an excessive increase of the number of required finite element nodes and - as a consequence - the associated system of equations would be very huge. The number of variables can be significantly reduced, if the temporal discretization by space-time finite elements is only performed with respect to a short time-slot. Then, an iterative method can be used to solve the dynamics of the system within the entire time frame by an individual consideration of successive time-slots. The methods that use finite elements for the time-discretization are known as space-time finite element methods.

In this work, the time-discretization is realized by an implicit direct finite difference scheme due to the simplicity and robustness of these methods. The term "direct" means that, in contrast to e.g. mode superposition methods, the system of equation will be not initially transformed into a different formulation before the numerical integration is performed. The finite difference scheme is characterized by the division of the entire time interval into a set of sub-intervals $\Delta t_{i}=\left[t_{i}, t_{i+1}\right]$. On the basis of these segments the solution is successively approximated at each instant of time $t_{i}$. Obviously, the computational effort of the time-integration is directly proportional to the number of time-steps. Therefore, the length of an sub-interval should be chosen in such a way that on the one hand the resultant approximation will be as good as possible and that on the other hand the required computational effort will be minimized. In this work, the time-steps $\Delta t_{i}$ are assumed to be constant with respect to a specific simulation experiment. The acoustic reaction of the first order linear subproblem is mainly induced by the harmonic stimulation at the oval window. Therefore, it seems to be a good idea to link the length of a sub-interval to the respective stimulation frequency. 
In many applications, explicit integration methods are preferred due to a potentially more effective computation at each time-step. But, it can be shown that the stability of explicit integration methods can only be guaranteed for intervals below an upper limit. This upper limit depends on each individual problem itself and it may be that the timesteps have to be chosen very small. In this context, a time integration technique is referred to as stable if the growth of an arbitrarily induced error is bounded. In contrast to explicit integration schemes, implicit methods are more robust in the face of the choice of the time-step. Commonly, implicit methods are not restricted to such an upper limit and they can therefore be considered as an unconditionally stable approach.

As already mentioned above, the spatial discretization of the first order problem results in a second order linear ordinary system of differential equations (cf. equation 3.87). It is always possible to transform such a second order system into a first order formulation by the introduction of new variables. On the basis of such a conversion, a standard approach for first order problems like the implicit backward Euler method, the Crank-Nicolson method or the Runge-Kutta method might be performed. These methods mainly differ from each other in regard to the degree of precision with which the solution might be approximated. But due to the doubling of the variables, which is accompanied with a significant higher computational effort and an increased requirement of memory, this kind of procedure is not implemented for the numerical integration of the first order problem.

Therefore, a direct discretization of the second order ordinary differential equation is preferred. This work uses an approach that was originally suggested by Houbolt [21]. The approximation technique from Houbolt is a second order method, which means that the error per time-step is of the order $\Delta t^{2}$. Furthermore, the scheme is numerically stable since it is an implicit approach. (cf. [3]) The integration scheme from Houbolt uses the following backward finite difference approximations for the first and second time-derivative:

$$
\begin{aligned}
& \dot{\mathbf{u}}_{t+\Delta t}=-\frac{1}{6 \Delta t}\left(2 \mathbf{u}_{t-2 \Delta t}-9 \mathbf{u}_{t-\Delta t}+18 \mathbf{u}_{t}-11 \mathbf{u}_{t+\Delta t}\right), \\
& \ddot{\mathbf{u}}_{t+\Delta t}=-\frac{1}{\Delta t^{2}}\left(\mathbf{u}_{t-2 \Delta t}-4 \mathbf{u}_{t-\Delta t}+5 \mathbf{u}_{t}-2 \mathbf{u}_{t+\Delta t}\right) .
\end{aligned}
$$

The semi-discretized formulation of the first order problem at time $t+\Delta t$ can be written as

$$
\mathbf{M} \ddot{\mathbf{u}}_{t+\Delta t}+\mathbf{H} \dot{\mathbf{u}}_{t+\Delta t}+\mathbf{K} \mathbf{u}_{t+\Delta t}=\mathbf{F}_{t+\Delta t} .
$$

By substituting the approximations 3.120 and 3.121 for the velocity-like vector $\dot{\mathbf{u}}_{t+\Delta t}$ and the acceleration-like vector $\ddot{\mathbf{u}}_{t+\Delta t}$, the fully discretized first order subproblem can be represented by the simple linear system of equations

$$
\mathbf{A} \mathbf{u}_{t+\Delta t}=\mathbf{b} .
$$

In this context, the matrix $\mathbf{A}$ can be evaluated to

$$
\mathbf{A}=\frac{2}{\Delta t^{2}} \mathbf{M}+\frac{11}{6 \Delta t} \mathbf{H}+\mathbf{K} .
$$


As it can be seen, it is sufficient to determine the matrix $\mathbf{A}$ only once at the beginning of the numerical time integration process due to the stationary character of the mass matrix $\mathbf{M}$, the damping matrix $\mathbf{H}$ and the stiffness matrix $\mathbf{K}$. The load term $\mathbf{b}$ can be calculated to

$$
\begin{gathered}
\mathbf{b}=\left(\frac{1}{\Delta t^{2}} \mathbf{M}+\frac{1}{3 \Delta t} \mathbf{H}\right) \mathbf{u}_{t-2 \Delta t}-\left(\frac{4}{\Delta t^{2}} \mathbf{M}+\frac{3}{2 \Delta t} \mathbf{H}\right) \mathbf{u}_{t-\Delta t} \\
+\left(\frac{5}{\Delta t^{2}} \mathbf{M}+\frac{3}{\Delta t} \mathbf{H}\right) \mathbf{u}_{t}+\mathbf{F}_{t+\Delta t}
\end{gathered}
$$

In contrast to the stationary matrix on the left hand side of equation 3.123, the load term $\mathbf{b}$ has to be updated at each time-step due to the dependency on the time-varying load vector $\mathbf{F}_{t+\Delta t}$ (which takes account of the predefined displacements of the oval window) and the inclusion of the state vectors $\mathbf{u}$ at the preceding three time-steps.

In literature, several other techniques for the numerical discretization can of course be found. For example, the Newmark-method and the Wilson-method (cf. e.g. [3]) are two other similar approaches that can also be used in the context of a linear second order ordinary differential equation.

\subsection{Linear Solver}

The discretization process of the first order system results in a sequence of linear systems of equations (cf. 3.123). Under the assumption of a constant time-step, the linear systems are characterized by a constant, large, sparse and non-symmetric matrix as well as a timevarying right hand side vector. In order to get a numerical solution of each system, the generalized minimal residual method (GMRES) is used due to its applicability for nonsymmetric systems. The iterative GMRES approach was originally developed by Saad and Schultz [40] and it is based on a projection method that approximates the solution within the so-called Krylov subspace.

The acoustically driven flow field can be achieved by solving the symmetric system of equations as described in chapter 3.4.3. Although the symmetry of the matrix allows the usage of more efficient methods (e.g. the Lanczos algorithm), the GMRES method is also used for solving the mean second order system. Due to its major importance for the numerical simulation process presented in this work, the main aspects of the GMRESmethod are briefly outlined in this chapter. The notation is orientated towards the original work from Saad and Schultz [40]. Further details to this subject can additionally be found in [39].

The speed of convergence of an iterative solver can be significantly enhanced by transforming the linear system of equations into a better conditioned system. The basic usage of a preconditioner in the context of the GMRES-method is described in section 3.6.2. In this work, an incomplete $L U$ decomposition is used as a preconditioner. The algorithm used for deducing such an incomplete factorization is presented in section 3.6.3. 


\subsubsection{Generalized Minimal Residual Method}

In general, the linear system of equation can be represented as

$$
\mathbf{A} \boldsymbol{x}=\boldsymbol{b}
$$

with $\mathbf{A} \in \mathbb{R}^{n \times n}$ being a regular matrix, $\boldsymbol{x} \in \mathbb{R}^{n}$ the vector of unknowns and $\boldsymbol{b} \in \mathbb{R}^{n}$ the right hand side vector. If $\boldsymbol{x}_{\mathbf{0}}$ denotes an initial guess of the solution, the error can be quantified by the residual

$$
\mathbf{r}_{0}=\boldsymbol{b}-\mathbf{A} x_{0}
$$

The GMRES-method aims to minimize the residual within the affine space $\boldsymbol{x}_{\mathbf{0}}+\mathcal{K}_{m}$ where $\mathcal{K}_{m}$ denotes the Krylov subspace which can be specified on the basis of the residual vector and the system matrix as

$$
\mathcal{K}_{m}\left(\mathbf{A}, \boldsymbol{r}_{\mathbf{0}}\right)=\operatorname{span}\left\{\boldsymbol{r}_{\mathbf{0}}, \mathbf{A} \boldsymbol{r}_{\mathbf{0}}, \mathbf{A}^{2} \boldsymbol{r}_{\mathbf{0}}, \ldots, \mathbf{A}^{m-1} \boldsymbol{r}_{\mathbf{0}}\right\}
$$

Initially, an orthonormal basis of the Krylov subspace is constructed by means of an orthogonal projection method from Arnoldi as described in algorithm 3.3 from line 8 to 13 , Having regard to the numerical stability, the algorithm adapts the modified Gram-Schmidt orthonormalization process. To further enhance the reliability of the orthonormalization process, the Gram-Schmidt based algorithm can, for example, be replaced by the more computationally intensive Householder orthogonalization (cf. [39]). It is important to note that the Hessenberg matrix $\overline{\mathbf{H}}^{[m]}$ can be extracted from Arnoldi's procedure as a byproduct of the orthogonalization process. Hessenberg matrices are characterized by their vanishing entries below the first subdiagonal. If the orthogonal basis vectors of the Krylov space $\mathcal{K}_{m}$ are combined into the matrix $\mathbf{V}^{[m]}$, the relation

$$
\mathbf{A} \mathbf{V}^{[m]}=\mathbf{V}_{m+1} \overline{\mathbf{H}}^{[m]}
$$

can be directly deduced from algorithm 3.3 . Furthermore it can be seen that the first basis vector $\boldsymbol{v}_{1}$ can be identified with the residual vector $\boldsymbol{r}_{\mathbf{0}}$. According to the equation

$$
\boldsymbol{x}=\boldsymbol{x}_{\mathbf{0}}+\mathbf{V}^{[m]} \boldsymbol{y}
$$

all elements $\boldsymbol{x}$ of the affine subspace can be expressed as a linear combination of the orthogonal basis functions by the use of an appropriate choice of the vector $\boldsymbol{y}$. In terms of a formal notation, the GMRES-method is based on the minimization of the function

$$
J(\boldsymbol{y}):=\|\boldsymbol{b}-\mathbf{A} \boldsymbol{x}\|_{2}=\left\|\boldsymbol{b}-\mathbf{A}\left(\boldsymbol{x}_{\mathbf{0}}+\mathbf{V}^{[m]} \boldsymbol{y}\right)\right\|_{2}
$$

where the approximation $\boldsymbol{x}$ should, by definition, belong to the affine Krylov subspace and can therefore be concretized by using equation 3.130 . In consideration of equations 3.127 


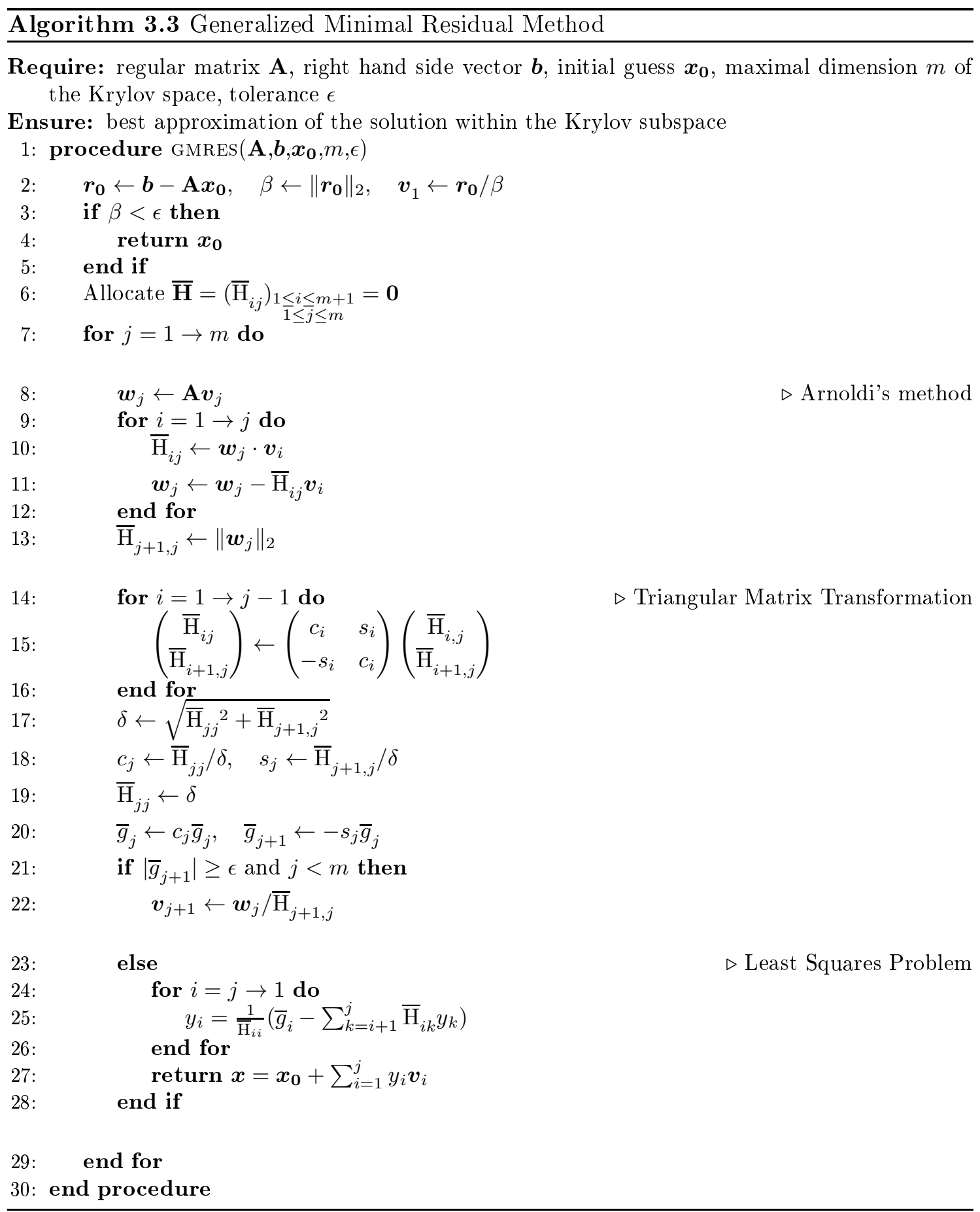


and 3.129, the expression within the norm of equation 3.131 can be transformed as

$$
\begin{aligned}
\boldsymbol{b}-\mathbf{A}\left(\boldsymbol{x}_{\mathbf{0}}+\mathbf{V}^{[m]} \boldsymbol{y}\right) & =\boldsymbol{r}_{\mathbf{0}}-\mathbf{A} \mathbf{V}^{[m]} \boldsymbol{y} \\
& =\beta \boldsymbol{v}_{1}-\mathbf{V}_{m+1} \overline{\mathbf{H}}^{[m]} \boldsymbol{y} \\
& =\mathbf{V}^{[m+1]}\left(\beta \boldsymbol{e}_{1}-\overline{\mathbf{H}}^{[m]} \boldsymbol{y}\right)
\end{aligned}
$$

where the notation $\beta=\left\|\boldsymbol{r}_{\mathbf{0}}\right\|_{2}$ is used for reasons of simplicity. Since the orthonormal matrix $\mathbf{V}^{[m+1]}$ has no effect on the euclidean norm, the minimal residual within the affine Krylov subspace can also be specified by the equivalent formulation

$$
\begin{aligned}
& \boldsymbol{x}^{[m]}:=\boldsymbol{x}_{\mathbf{0}}+\mathbf{V}^{[m]} \boldsymbol{y}^{[m]}, \text { where } \\
& \boldsymbol{y}^{[m]}:=\operatorname{argmin}_{\boldsymbol{y}}\left\|\beta \boldsymbol{e}_{1}-\overline{\mathbf{H}}^{[m]} \boldsymbol{y}\right\|_{2} .
\end{aligned}
$$

The advantage over the initial formulation of the the minimization problem (cf. equation 3.131) lies in the more efficient computation in combination with the simultaneous control of the residual size. This effective computation can be achieved through a transformation of the Hessenberg matrix

$$
\overline{\mathbf{H}}^{[m]}=\left(\begin{array}{cccc}
\overline{\mathrm{H}}_{11} & \overline{\mathrm{H}}_{12} & \cdots & \overline{\mathrm{H}}_{1 m} \\
\overline{\mathrm{H}}_{21} & \overline{\mathrm{H}}_{22} & \cdots & \overline{\mathrm{H}}_{2 m} \\
& \overline{\mathrm{H}}_{32} & \cdots & \overline{\mathrm{H}}_{3 m} \\
& & \ddots & \vdots \\
& & & \overline{\mathrm{H}}_{m+1, m}
\end{array}\right)
$$

into an upper triangular form by performing plane rotations in order to successively eliminate the entries $\overline{\mathrm{H}}_{i+1, i}$ in an iterative process. In this context, each rotation matrix can be written as

$$
\boldsymbol{\Omega}_{i}=\left(\begin{array}{cccccccc}
1 & & & & & & & \\
& \ddots & & & & & & \\
& & 1 & & & & & \\
& & & c_{i} & s_{i} & & & \\
& & & -s_{i} & c_{i} & & & \\
& & & & 1 & & \\
& & & & & & \ddots & \\
& & & & & & & 1
\end{array}\right)
$$

where the $2 \times 2$-block that causes the rotation is positioned at the $i$-th row and $i$-th column. The two values $c_{i}$ and $s_{i}$ result from the current state of the matrix that depends on the rotations performed before. If this current state is described by

$$
\overline{\mathbf{H}}^{[m, i]}:=\boldsymbol{\Omega}_{i} \cdots \boldsymbol{\Omega}_{1} \overline{\mathbf{H}}^{[m]}
$$


the two values can be calculated to

$$
\begin{aligned}
& c_{i}=\frac{\overline{\mathrm{H}}_{i+1, i}^{[m, i]}}{\sqrt{\left(\overline{\mathrm{H}}_{i i}^{[m, i]}\right)^{2}+\left(\overline{\mathrm{H}}_{i+1, i}^{[m, i]}\right)^{2}}}, \\
& s_{i}=\frac{\overline{\mathrm{H}}_{i i}^{[m, i]}}{\sqrt{\left(\overline{\mathrm{H}}_{i i}^{[m, i]}\right)^{2}+\left(\overline{\mathrm{H}}_{i+1, i}^{[m, i]}\right)^{2}}} .
\end{aligned}
$$

Of course, these transformations must also be applied to the term $\beta \boldsymbol{e}_{1}$ in order to get an equivalent restatement of the euclidean norm. After $m$ rotations, this vector and the upper triangular matrix can be represented as

$$
\begin{aligned}
\overline{\boldsymbol{g}} & :=\boldsymbol{\Omega}_{m} \cdots \boldsymbol{\Omega}_{1} \beta \boldsymbol{e}_{1}, \\
\overline{\mathbf{R}} & :=\boldsymbol{\Omega}_{m} \cdots \boldsymbol{\Omega}_{1} \overline{\mathbf{H}}^{[m]}=\overline{\mathbf{H}}^{[m, m]} .
\end{aligned}
$$

Due to the invariance of the euclidean norm to the rotations $\boldsymbol{\Omega}_{i}$, the minimization problem 3.134 can therefore be written as

$$
\min _{\boldsymbol{y}}\left\|\beta \boldsymbol{e}_{1}-\overline{\mathbf{H}}^{[m]} \boldsymbol{y}\right\|_{2}=\min _{\boldsymbol{y}}\left\|\overline{\boldsymbol{g}}-\overline{\mathbf{R}}^{[m]} \boldsymbol{y}\right\|_{2} .
$$

In this case, the residual can be minimized by determining the unknown vector $\boldsymbol{y}$ in such a way that the first $m$ entries of the resultant vector within the norm vanish. This is possible due to the triangular configuration of the matrix $\overline{\mathbf{R}}^{[m]}$. But the last entry of the resultant vector can not be affected by $\boldsymbol{y}$, since the last row of the matrix consists only of zeros. As a consequence, the euclidean norm of the minimal residual must be equal to the absolute value of the last entry $\bar{g}_{m+1}$.

As illustrated in algorithm 3.3 from line 14 to line 22 and as described by Saad [39], the respective rotations can be performed at each iteration separately for each new column of the matrix $\overline{\mathbf{H}}^{[m]}$ without additional computational effort. Therefore, the decrease of the residual error can be directly monitored at each iteration without calculating the current approximation. If the residual error falls below the predefined tolerance, the approximation can be calculated by determining $\boldsymbol{y}^{[\mathrm{m}]}$ (as mentioned above) and inserting these coefficients into the linear combination 3.133 (cf. algorithm 3.3 from line 24 to 27).

Apart from numerical errors, the GMRES-procedure yields the exact solution after $n$ iterations at the latest. But the computational effort as well as the memory requirements are directly related to the maximal dimension of the Krylov subspace and therefore also to the maximal number of iterations. The demands on the memory can be limited by predefining the maximal dimension of the Krylov space. If the residual error still exceeds the predefined tolerance, the GMRES-algorithm might be start again by taking the current approximation as the initial guess of the subsequent run. This approach is known as restarted GMRES. This restarted version was also used for the numerical simulations performed in this work, where the maximal dimension of the Krylov subspace was set to 30. 


\subsubsection{Preconditioning}

The efficiency of the iterative solvers can be significantly improved by using a preconditioner. The preconditioner should transform the linear system into an equivalent formulation that is characterized by a better rate of convergence. The transformation can usually performed by the application of a left preconditioner $\mathbf{P}_{l}$ and/or a right preconditioner $\mathbf{P}_{r}$. Then, the preconditioned system of equations can be written as

$$
\mathbf{P}_{l} \mathbf{A} \mathbf{P}_{r} \boldsymbol{x}_{P}=\mathbf{P}_{l} \boldsymbol{b} \quad \text { where } \quad \boldsymbol{x}_{P}=\mathbf{P}_{r}^{-1} \boldsymbol{x} .
$$

Usually, the preconditioners are constructed in such a way that the resultant matrix of the preconditioned system approximates the identity matrix as well as possible; in short $\mathbf{P}_{l} \mathbf{A} \mathbf{P}_{r} \approx \mathbf{I}$. But, in order to take profit from the preconditioning process, the construction of the transformation matrices should involve a minimum of computational effort. In the context of the GMRES-method, the right hand side preconditioning has advantages over the left approach because of two different aspects. Firstly, the current norm of the residual (computed at each iteration) refers, in contrast to the left preconditioning approach, not to the preconditioned system but rather to the initial system. This point might be important, since the stop criterion is normally specified with respect to the initial system. Secondly, the right hand side application allows a variation of the preconditioner at each iteration which opens possibilities for a better influence on the iterative progress. A formal and more detailed consideration of preconditioning can, for example, be found in [39].

For the implementation of a right preconditioner the algorithm 3.3 has to be modified at two lines. At line 8, the precondition matrix $\mathbf{P}_{r}$ have to be inserted at the right hand side and, at line 27, the approximation must be calculated according to

$$
\boldsymbol{x}=\boldsymbol{x}_{\mathbf{0}}+\mathbf{P}_{r} \mathbf{V}^{[m]} \boldsymbol{y} .
$$

\subsubsection{Incomplete LU decomposition}

This work uses an incomplete $L U$ decomposition (ILU) as a preconditioner. As the name implies, this incomplete factorization is based on the well-known LU decomposition, which splits the original matrix into a product of a lower triangular and an upper triangular matrix. The execution of the full decomposition process is computationally intensive and although the matrix $\mathbf{A}$ is only sparsely populated, it can be expected that the resultant factorization consists of two dense (triangular) matrices. In contrast, the incomplete LU decomposition can be written as

$$
\mathbf{A}=\mathbf{L} \mathbf{U}+\mathbf{R}
$$

with $\mathbf{L}$ being a sparse lower triangular matrix, $\mathbf{U}$ a sparse upper triangular matrix and $\mathbf{R}$ the residual that takes the resultant error into account. In literature, different approaches can be found for establishing different kinds of such a decomposition. 


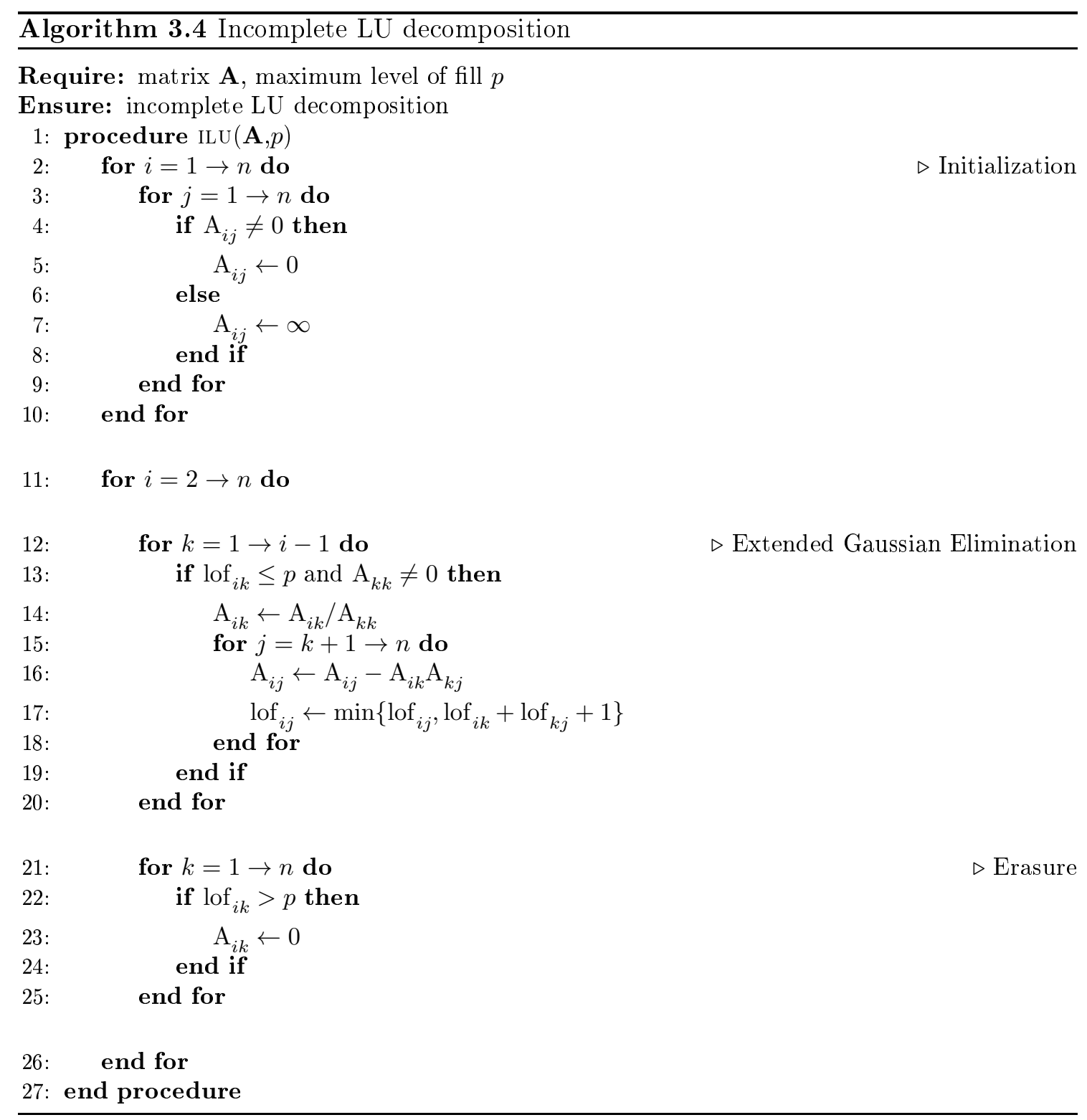


In this work, the so-called $I L U(p)$-procedure is adopted to calculate an appropriate preconditioner. As illustrated in algorithm 3.4 from line 12 to 20 , this approach is, in principle, based on the Gaussian elimination which is supplemented by an additional marking strategy in order to specify the non-zero pattern of the matrices. The Gaussian algorithm successively eliminates the entries below the main diagonal by adding an appropriate multiple of row $k$ to row $i$ for all rows $i=k+1, \ldots, n$. This method yields the upper triangular matrix $\mathbf{U}$ as well as the lower triangular matrix $\mathbf{L}$ as a byproduct. Both of them can be stored in a single matrix, since the entries of the main diagonal of the lower triangular matrix are equal to one and do not need to be stored explicitly. The marking strategy is based on the so-called level of fill value lof ${ }_{i j}$, which can be simultaneously calculated for each entry. Initially, almost all entries of the matrix have a level of fill of $\infty$ except for the non-zero entries as well as the entries upon the main diagonal, which are labeled with the level 0 (cf. algorithm 3.4 from line 2 to 10 ). Each modification of a specific entry is accompanied with an update of its level of fill. According to line 17, the new level of fill can be calculated as

$$
\operatorname{lof}_{i j}=\min \left\{\operatorname{lof}_{i j}, \operatorname{lof}_{i k}+\operatorname{lof}_{k j}+1\right\} \text {. }
$$

Once a whole row has been updated, all entries, whose level of fill exceeds the predefined maximum level $p$, are not taken into account by the incomplete LU decomposition and are therefore erased (cf. algorithm 3.4 from line 21 to 251). The analysis and a detailed description of the incomplete LU decomposition can for example be found in [39].

In this work, the GMRES-method was transformed by using a preconditioner generated by the ILU(2) method. It has been shown that an higher maximum level normally induces a longer calculation time.

\subsection{Sine-Cosine Decomposition}

For reasons of convenience, the equilibrium state is represented by using the sine-cosine decomposition as introduced in chapter 2.3.4. Since the sine-cosine decomposition is identical with the first non-constant term of the Fourier series, the coefficients $\boldsymbol{u}^{(\sin )}$ and $\boldsymbol{u}^{\text {(cos) }}$, which represent the steady state of the time-varying solution vector $\boldsymbol{u}$, can be determined according to the relations

$$
\begin{aligned}
& \boldsymbol{u}^{(\sin )}=\frac{2}{N} \sum_{n=0}^{N-1} \boldsymbol{u}\left(t^{*}+n \Delta t\right) \sin \left(2 \pi f\left(t^{*}+n \Delta t\right)\right), \\
& \boldsymbol{u}^{(\cos )}=\frac{2}{N} \sum_{n=0}^{N-1} \boldsymbol{u}\left(t^{*}+n \Delta t\right) \cos \left(2 \pi f\left(t^{*}+n \Delta t\right)\right)
\end{aligned}
$$

where $N:=T / \Delta t$ and $t^{*}$ is the time at which the equilibrium state is achieved. The velocity coefficients $\boldsymbol{v}^{(\sin )}$ and $\boldsymbol{v}^{(\cos )}$, the pressure coefficients $p^{(\sin )}$ and $p^{(\cos )}$, as well as the displacement coefficients of the basilar membrane $\xi^{(\sin )}$ and $\xi^{(\cos )}$ can be extracted in consideration of equation 3.88 . 
With the help of the sine-cosine decomposition, the time-averaged load vector of the second order system 3.110 can be determined in a simplified way. By substituting the sine-cosine representation for the velocity and pressure functions and applying the meanoperator, the mass source distribution 2.47 can be written as

$$
\tilde{m}=-\frac{1}{2 c_{0}^{2}} \operatorname{div}\left(p^{(\cos )} \boldsymbol{v}^{(\cos )}+p^{(\sin )} \boldsymbol{v}^{(\sin )}\right) .
$$

In addition, the force source distribution 2.49 can be expressed in terms of the sine- and cosine-coefficients as

$$
\tilde{\boldsymbol{f}}=-\frac{\pi f}{c_{0}^{2}}\left(p^{(\cos )} \boldsymbol{v}^{(\sin )}-p^{(\sin )} \boldsymbol{v}^{(\cos )}\right)-\frac{\rho^{(0)}}{2}\left(\left(\operatorname{grad} \boldsymbol{v}^{(\cos )}\right) \boldsymbol{v}^{(\cos )}+\left(\operatorname{grad} \boldsymbol{v}^{(\sin )}\right) \boldsymbol{v}^{(\sin )}\right)
$$

Since the boundary condition 2.52 is also based on the results of the first order equilibrium state, it is convenient to use its simplified representation which is given as

$$
\boldsymbol{v}^{(d c)}=-\frac{1}{2 \rho^{(0)} c_{0}^{2}}\left(p^{(\cos )} \boldsymbol{v}^{(\cos )}+p^{(\sin )} \boldsymbol{v}^{(\sin )}\right)+\frac{1}{4 \pi f} \operatorname{rot}\left(\boldsymbol{v}^{(\cos )} \times \boldsymbol{v}^{(\sin )}\right) .
$$




\section{Chapter 4}

\section{Results}

In this chapter the numerical results of the computational model, introduced in this work, are presented. In order to ensure the correct functionality of the model, the numerical results are compared to experimental studies, measurements and analytical estimates.

The accuracy of the first order system is crucial for the correctness of the results of the second order system, which provides estimations for the occurrence of acoustically driven flows within the inner ear. Therefore, the first part of this chapter (cf. section 4.1) is concerned with an accurate verification of this acoustic system. It is analyzed under an harmonic stimulation of the oval window at different frequencies, amplitudes and outer hair cell activity parameters.

Particular attention is paid to the equilibrium state of the first order system, since it is the basis for the numerical calculation of acoustic streaming. This steady state is achieved within a short time frame. The transient effect is considered in section 4.1.1. Today, it is well accepted that the motions of the basilar membrane can be characterized on the basis of a typical traveling wave pattern. The basilar membrane motions arise, inter alia, through the hydrodynamic forces as well as the outer hair cell driven forces, which are both analyzed in section 4.1.2. The resultant displacements in comparison to the traveling wave pattern of the cochlear partition are reviewed in section 4.1.3. Furthermore, it has been shown that some boundary layer effects are mainly responsible for acoustically driven flows. In section 4.1.4, the motion within this thin boundary layer are therefore compared with an analytical approximation developed by Lighthill [25].

As discussed in chapter 2.3.3, the motility of the outer hair cells can be controlled by the activity parameter $\lambda$. The outer hair cell motility is modeled in such a way that the resultant forces (that act on the cochlear partition) partially eliminate the positional damping of the basilar membrane. As a result, an activity parameter greater than one induces potentially a local negative damping. Due to the linearization of the sigmoidfunction, the natural limitation of the outer hair cell force can not be taken into account by the model presented in this work. Therefore, in order to maintain the stability of the present linear model, the activity parameter should be smaller than one. If the activity 
parameter is set to zero, the outer hair cell motility will not be taken into account by the simulation process and the computational model can be referred to as passive. In the context of this work the term active, in contrast, is associated with $\lambda=0.9$ which ensures the stability of the system on the one hand and on the other hand provides a significant amplification of the cochlear displacement as it can be seen in this chapter. Unless otherwise indicated, the amplitude of the oval window displacement is set to $10 \mathrm{~nm}$.

The second part of this chapter 4.2 deals with the time-averaged second order system that yields a first order approximation of the acoustic streaming flow field. In this context different physical mechanisms are considered that are responsible for the occurrence of acoustic streaming. First of all, the distinction between the Lagrangian and the Eulerian specification of the acoustic field yields a mean flow field that appears without taking the second order system into account (cf. section 4.2.1). As discussed in chapter 4.2.2, it becomes apparent that this acoustic driven flow field transports mass. Therefore, the transported mass must be released at regions where the acoustic field dissipates. This phenomenon is reflected in the mass source distribution that appears at the right hand side of the secondary mass conservation equation. The mass source driven second order mean flows are reviewed in section 4.2.2. In a similar same way, also momentum can be transported by the acoustic field which results in a virtual force source distribution within the second order system. The impact of this force distribution on inducing acoustically driven flows is discussed in section 4.2.3. As already mentioned above, it turned out that the most important cause for acoustic streaming field can be associated with the forces within the thin boundary layer next to the basilar membrane. Therefore, the stresses within this boundary layer are compared with Lighthill's approximations. The resultant second order flow field is described and evaluated in the last section 4.2.4. In this context, the flow field is compared with experiments made by Tonndorf [48] and the magnitude of the resultant velocities are contrasted with the analytical results from Lighthill [25].

\subsection{Harmonic Stimulation of the First Order System}

\subsubsection{Initial Transient Effect}

In all experiments performed in this work, the computational model is stimulated by an harmonic excitation of the oval window. At the beginning of each experiment, the model is in a resting state, which means that the pressure of the fluid is constant over the whole domain and that there are no motions with respect to the velocity of the fluid and the displacement of the cochlear partition.

Before the system achieves a steady state, it takes about 6 to 16 periods in the context of the numerical simulations presented here. The duration of this transient effect depends mainly on the outer hair cell activity parameter due to an increased mutual influence between the fluid, the basilar membrane and the outer hair cell motility. The accuracy of the oscillatory equilibrium state can be further enhanced by extending the duration of this initial time frame. 
The figures of appendix A.1 illustrate the first two periods of this transient effect at a stimulation frequency of $1024 \mathrm{~Hz}$ in the passive cochlea model. After a quarter period, the velocity of the sinusoidal stimulation at the oval window is maximal and an inward directed motion can be observed as shown in figure A.1. As a result of this inward stapes displacement, the adjacent pressure of the fluid within the scala vestibuli increases. In contrary, the velocity of the fluid at the round window is not prescribed by a Dirichlet boundary condition and the motions are completely flexible. As a consequence, the pressure next to the round window is nearly time-invariant and its amount is the ambient value. Without loss of generality, the ambient pressure of the computational model is chosen to be zero for the purpose of simplification. (In reality, the ambient pressure is approximately equal to the atmospheric pressure, since the oval window separates the scala tympani from an air-filled space within the middle ear. (cf. [13]))

Due to the pressure-difference across the cochlear partition, a force is exerted upon the basilar membrane according to equation 2.33. This pressure-driven force causes instantly a displacement of the cochlear partition, which also begins to vibrate according to its local physical properties (stiffness, damping, mass, width, ...) and the forces acting on the basilar membrane. It can be clearly seen that the displacement, in turn, significantly influences the velocity field of the fluid.

While the wave front of the pressure propagates along the longitudinal axis, the velocity of the oval window displacement decreases which induces a negative excess pressure at the adjacent fluid layers as shown in figure A.2. The negative pressure difference at the base of the basilar membrane in combination with the elastic reaction of basilar membrane causes, in turn, a local upward directed displacement of the cochlear partition.

As illustrated by the following figures (A.3 A.8), this interplay between the pressure differences, fluid motions and basilar membrane oscillations results in a typical fluid-structure coupled wave motion along the cochlear partition up to a point of dissipation. In this context, a distinction between the pressure wave within the fluid and the displacement wave on the cochlear partition can be drawn (cf. [38]).

\subsubsection{Forces acting on the Basilar Membrane}

After achieving a steady state of oscillation, the system can be analyzed on the basis of the sine-cosine representation or, alternatively, the amplitude-phase description as described in chapter 2.3 .4 and 3.7 .

Figure 4.1](a) illustrates the dynamics of the pressure above and below the cochlear partition using the sine-cosine representation. First of all, it can be noted that the excess pressure vanishes at the base of the scala tympani over the whole period. In contrast, the pressure at the base of the scala vestibuli varies due to the excitation of the oval window. The hydrodynamic forces can be characterized by its wavelike variation along the basilar membrane. It can be observed that the wavelength decreases from base to the place where the wave dissipates. Except for the basal region, the pressure above and below the cochlear partition are, in a certain sense, opposite to each other. More specifically, a 


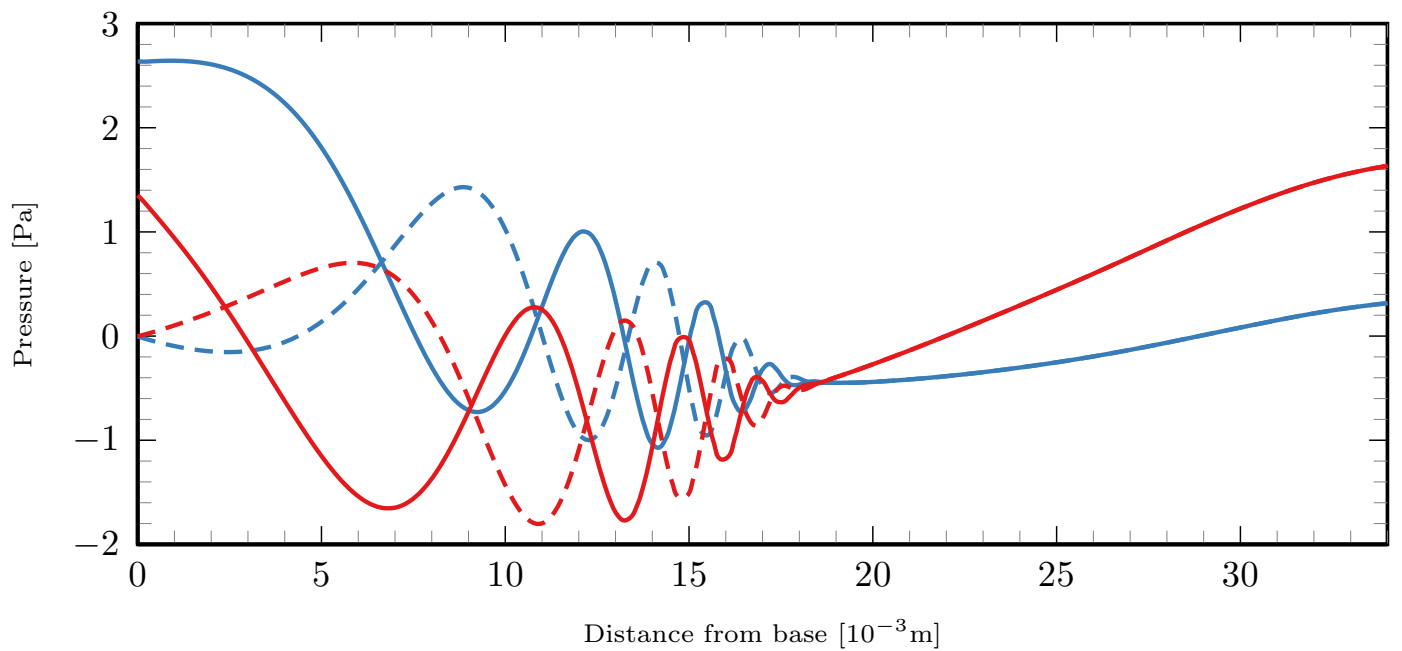

(a)

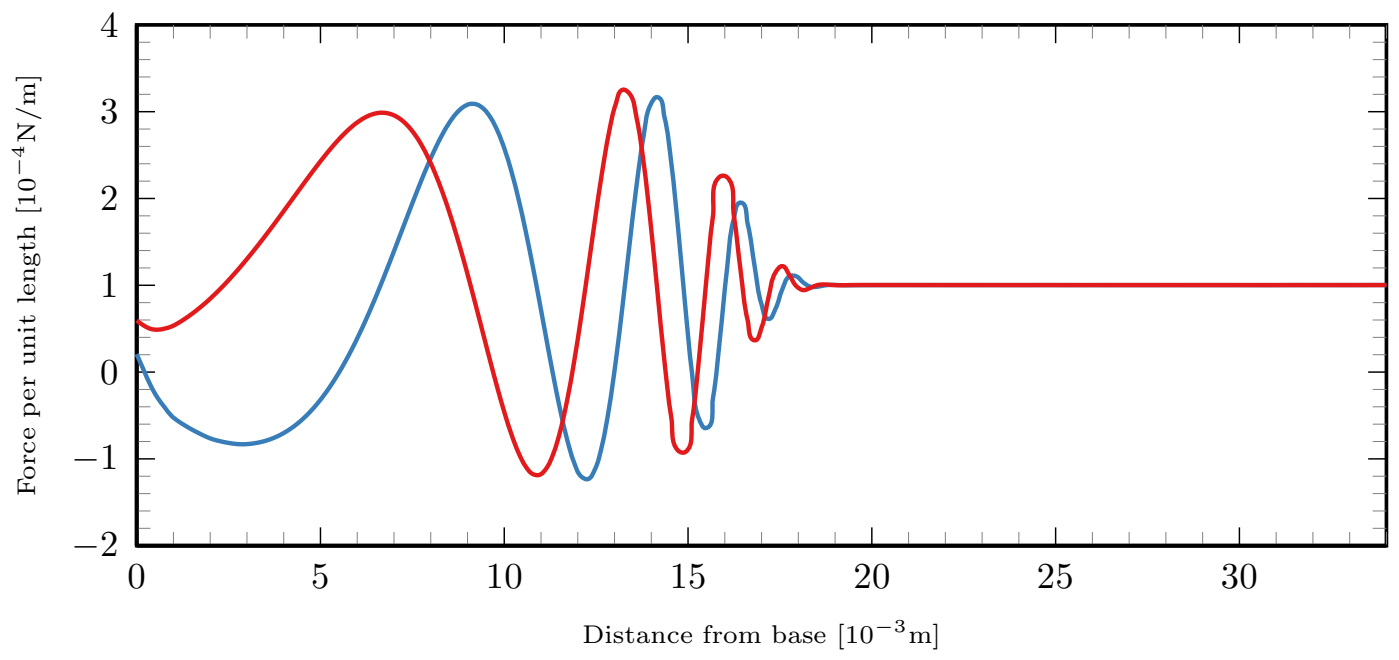

(b)

Figure 4.1: (a) Pressure above and below the basilar membrane at a stimulation frequency of 1024Hz in the passive cochlea model: $p_{+}^{(\cos )}$ (red line); $p_{-}^{(\cos )}$ (red dashed line); $p_{+}^{(\sin )}$ (blue line); $p_{-}^{(\sin )}$ (blue dashed line). (b) Resultant forces per unit length acting on the basilar membrane: $f_{p}{ }^{(\cos )}$ (red line); $f_{p}{ }^{(\sin )}$ (blue line). 


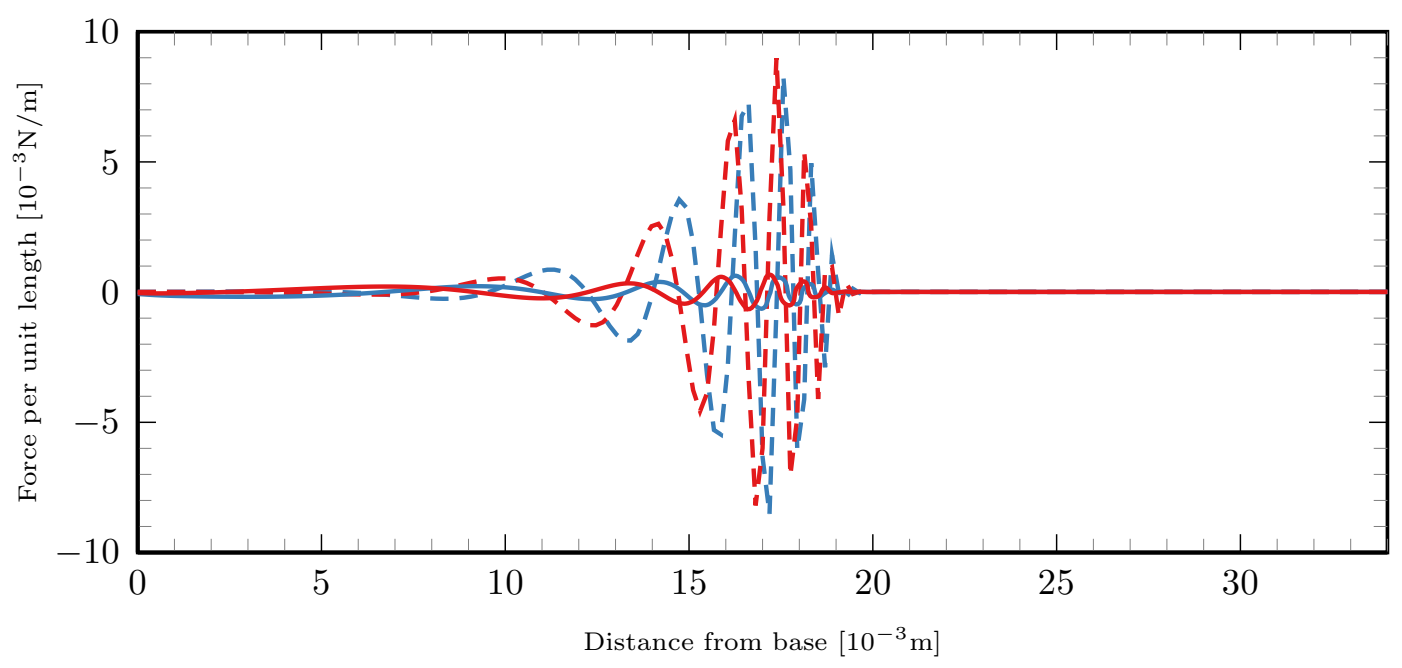

Figure 4.2: Comparison between the motility driven forces $\vartheta$ of the outer hair cells and the hydrodynamic forces $f_{p}$ at a stimulation frequency of $1024 \mathrm{~Hz}$ in the active cochlea model: $f_{p}{ }^{(\cos )}$ (red line); $f_{p}{ }^{(\sin )}$ (blue line), $\vartheta^{(\cos )}$ (red dashed line); $\vartheta^{(\sin )}$ (blue dashed line).

local maximum of the pressure on the upper side of the basilar membrane (at a specific time) is accompanied with a local minimum on the lower side and vice versa. Furthermore, it can be seen that the propagation of the pressure wave along the cochlear partition is characterized by a decreasing phase velocity.

As mentioned above, this pressure wave arises through the interaction between the fluid and the physical properties of the basilar membrane. The basilar membrane is stimulated by the pressure driven forces acting on the partition. According to equation 2.33 the hydrodynamic forces per unit length, illustrated in figure 4.1.(b), are proportional to the pressure differences and the width of the basilar membrane at each location. Similar to the individual pressure forces, the combined hydrodynamic force can also be characterized by its wavelike propagation along basilar membrane in combination with a decreasing wavelength and phase velocity.

Beside these hydrodynamic forces, the amplification mechanism based on the outer hair cell motility causes an additional internal force in the active cochlea model. Figure 4.2 illustrates these internal forces at equilibrium state that arise from a stimulation frequency of $1024 \mathrm{~Hz}$. In contrast to the hydrodynamic forces, the envelope of this motility driven force is characterized by a significant increase up to a certain place where the force reaches its maximum. At this location, the internal outer hair cell force is considerably greater than the external pressure force. Behind this point, the force dissipates within a short distance. Furthermore, it can be observed that both forces have a phase difference of about 90 degree. 


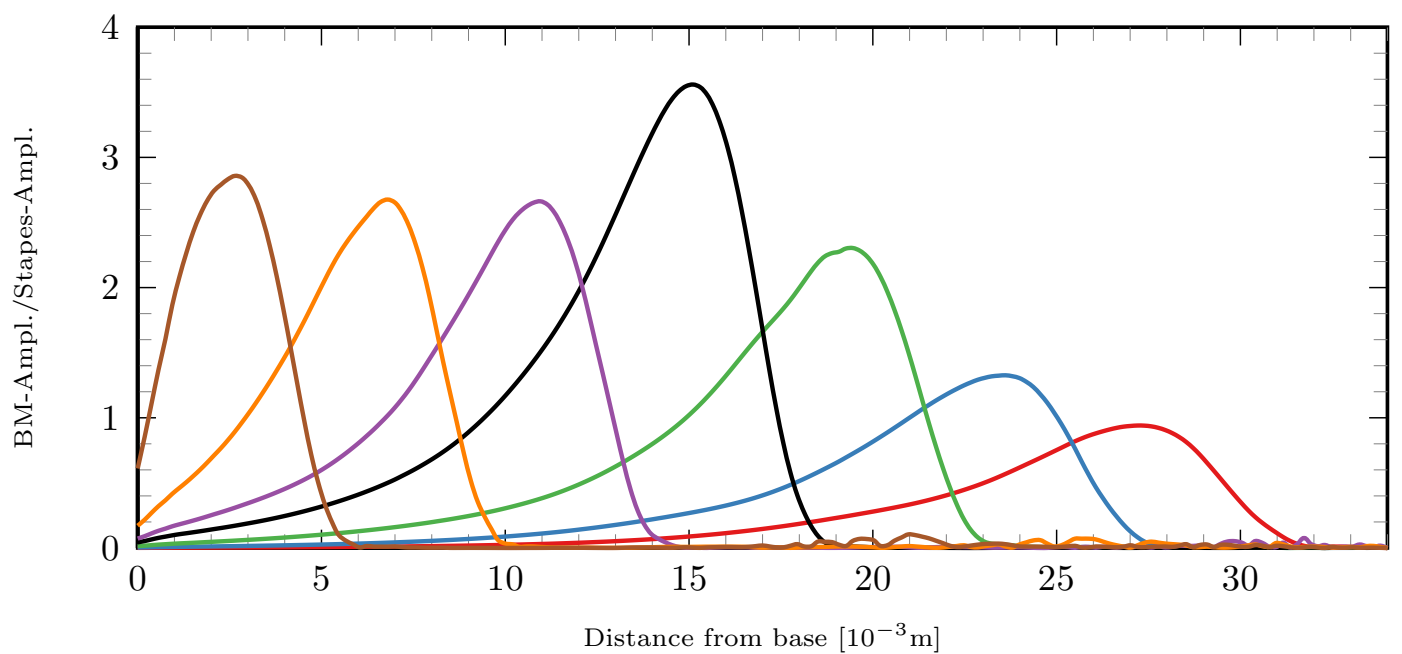

(a)

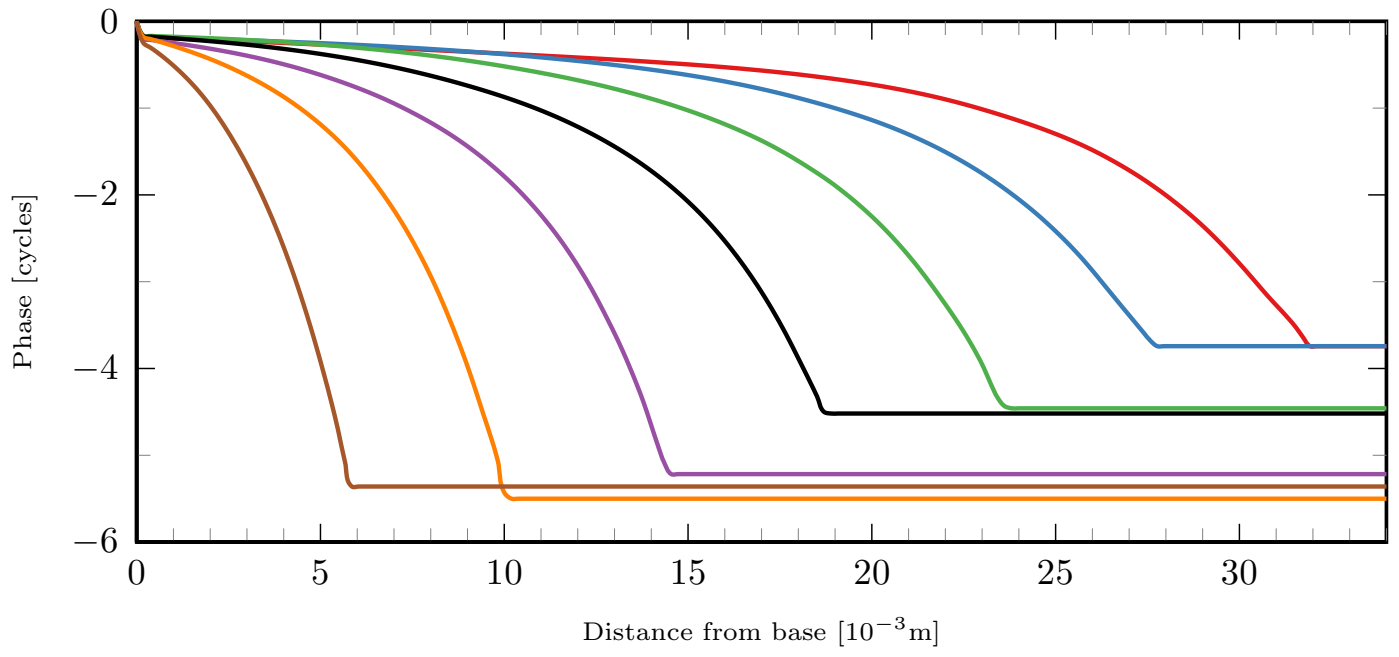

(b)

Figure 4.3: Envelopes (a) and phases (b) of the traveling wave in the passive cochlea model computed at constant stape displacement for a set of different frequencies: $128 \mathrm{~Hz}$ (red line), $256 \mathrm{~Hz}$ (blue line), 512 Hz (green line), 1024Hz (black line), 2048Hz (purple line), 4096Hz (orange line), 8192Hz (brown line). 


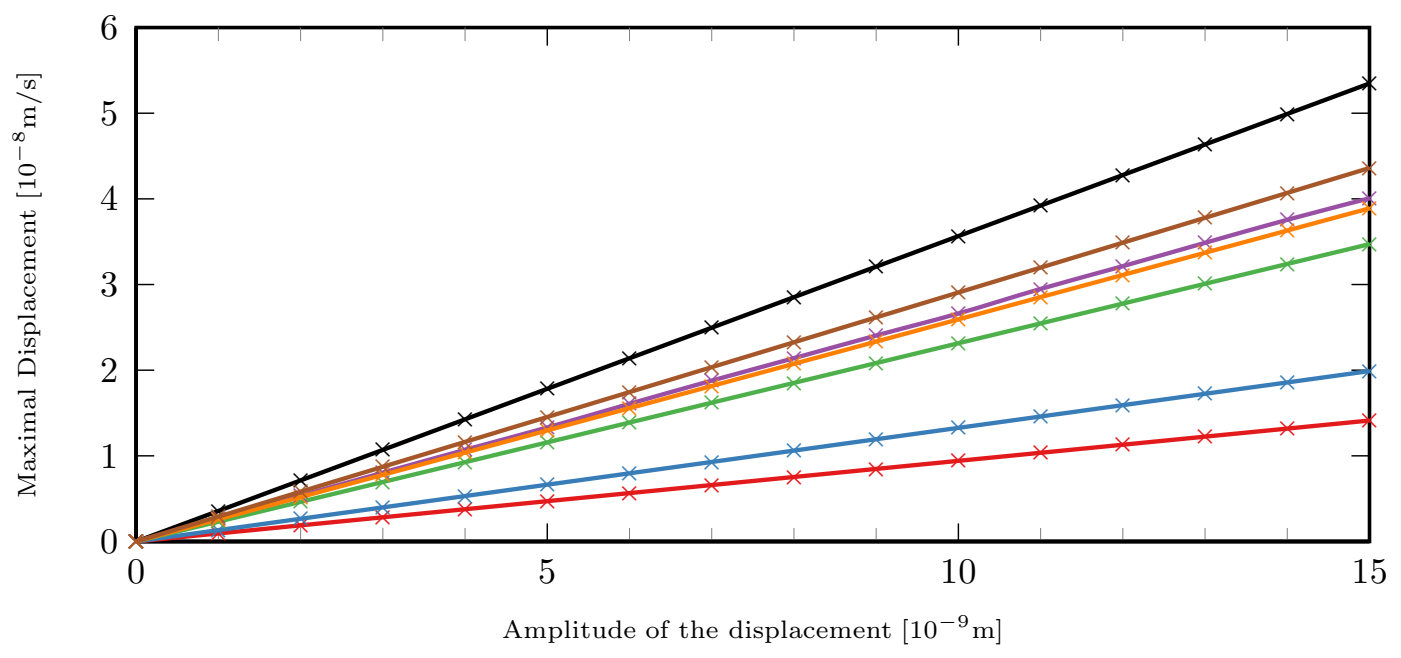

(a)

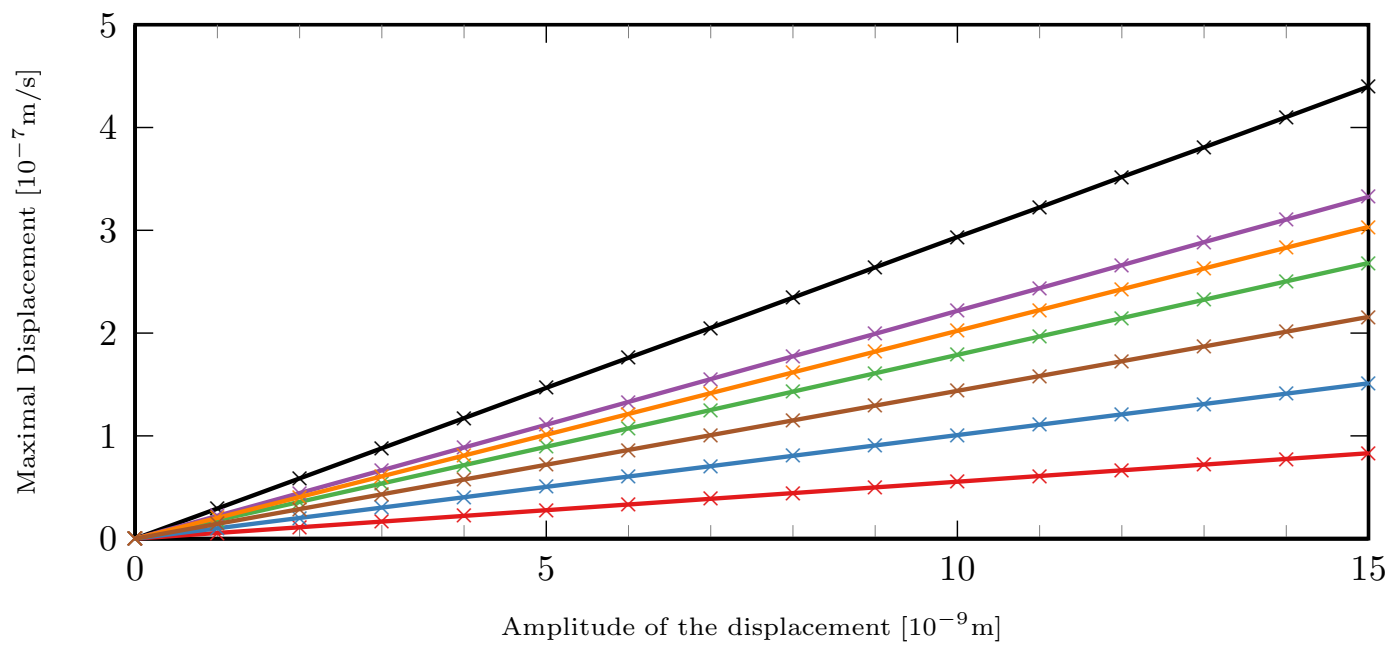

(b)

Figure 4.4: Maximal displacement of the basilar membrane in the passive cochlea model $(a)$ and the active cochlea model (b) at different stimulation amplitudes and frequencies: $128 \mathrm{~Hz}$ (red line), $256 \mathrm{~Hz}$ (blue line), $512 \mathrm{~Hz}$ (green line), $1024 \mathrm{~Hz}$ (black line), $2048 \mathrm{~Hz}$ (purple line), $4096 \mathrm{~Hz}$ (orange line), 8192Hz (brown line). 


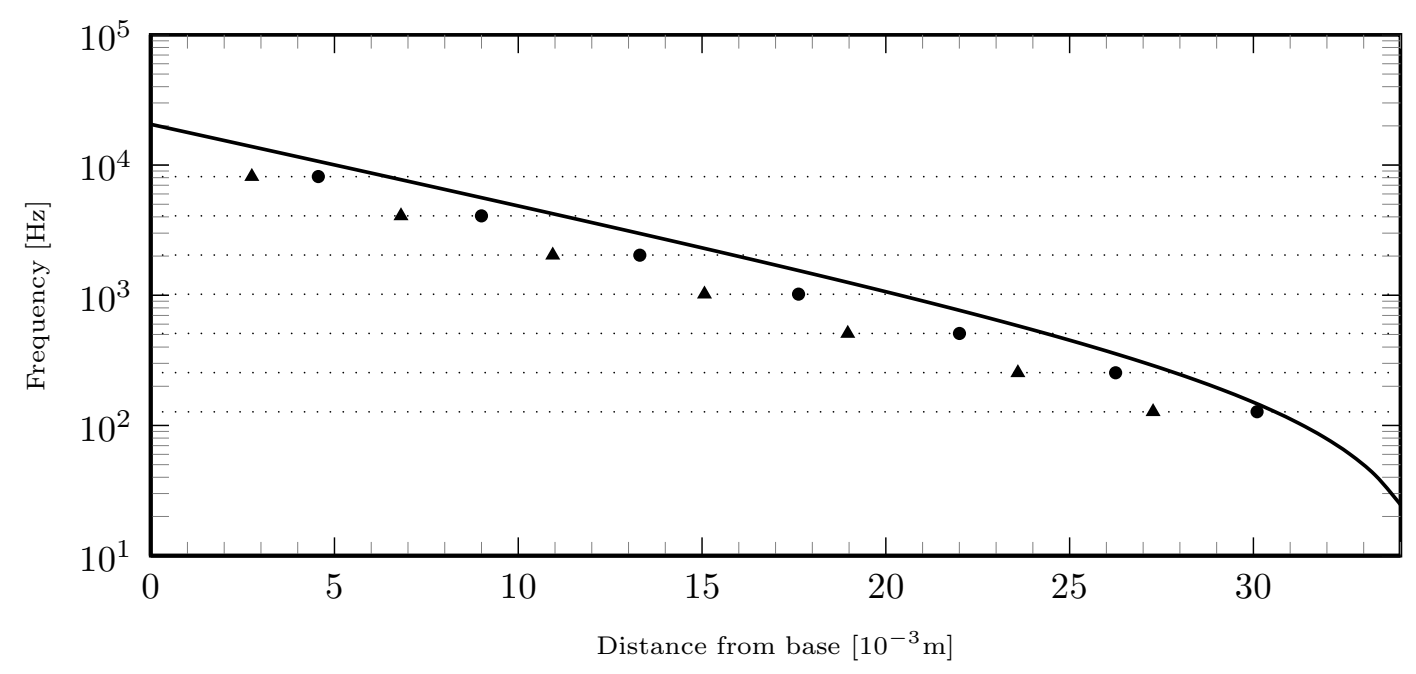

Figure 4.5: The frequency-position map (solid line) from Greenwood [18] assigns the characteristic places to their corresponding stimulation frequency. The triangles illustrate the characteristic places resulting from the passive cochlea model whereas the dots represent the characteristic places in the active case.

\subsubsection{Traveling Wave on the Basilar Membrane}

The pressure waves are the result of the interplay between the fluid and the cochlear partition. Today, it is well accepted that the dynamics of the basilar membrane can be described as a traveling wave. The term "traveling wave" is associated with a characteristic displacement pattern of the cochlear partition. This pattern is characterized by following properties (cf. [38, 13]):

- The amplitude of the traveling wave increases until a point of maximal displacement (peak) is reached. Often, this location is referred to as characteristic place. Behind this place, the amplitude falls to zero within a short distance.

- The phase of the oscillatory displacements decreases monotonically along the basilar membrane. This phase lag may amount to up to several cycles until the traveling wave dissipates.

- The traveling wave is caused by (passive) mechanical interactions between the fluid and the structures of the cochlea. This means that the traveling wave is, in particular, not a result of any other internal (physiological) processes.

- In contrast to the acoustic waves, which propagate at speeds of about $1500 \mathrm{~m} / \mathrm{s}$, the velocity of the traveling wave motion of the basilar membrane is significantly slower.

The present computational model has been performed for a set of different frequencies, stimulation amplitudes and outer hair cell activity parameters. 
Passive Cochlea Model Figure 4.3 shows the envelopes and phases of the traveling waves of the basilar membrane at stimulation frequencies of $128 \mathrm{~Hz}, 256 \mathrm{~Hz}, 512 \mathrm{~Hz}, 1024 \mathrm{~Hz}$, $2048 \mathrm{~Hz}, 4096 \mathrm{~Hz}$ and $8192 \mathrm{~Hz}$ in the passive case. The sine- and cosine-coefficients of the associated displacements can be found in appendix A.3. It becomes apparent that the resultant displacements of the cochlear partition meet the requirements of the typical traveling wave motion as described above. The phase lag adds up to just under four cycles at the lower frequencies and increases to more than five cycles at higher frequencies.

As it can be clearly seen, the longitudinal position of the characteristic place depends mainly on the frequency. Greenwood [18] empirically developed a functional relationship that assigns the characteristic places to their corresponding stimulation frequency. With regard to the human cochlea this frequency-position map is given by

$$
f=350 \cdot\left(10^{\frac{2.1}{l_{b m}}\left(x-l_{b m}\right)}-0.85\right)
$$

where $l_{b m}$ denotes the length of the basilar membrane. Figure 4.5 shows that the maximal displacements of the traveling wave are located up to about $5 \mathrm{~mm}$ closer to the base in the passive cochlea model than in the case of Greenwood's examination.

There is a broad consensus that the energy that causes the traveling wave motion is mainly transmitted by the fluid and only insignificantly by the basilar membrane itself (cf. [38, 28]). According to equation 2.32, longitudinal coupling can only be provided by the shearing resistance term. The results of the computations have also shown that this term has no significant influence on the shape of the traveling waves.

According to experimental data at basal sites of the cochlea, the amplitude of the basilar membrane displacement increases linearly with the stimulation intensity below a specific sound pressure level. But above this specific stimulation level, it can be observed that the growth of cochlear response is not as high as the increase of the stimulation level. (cf. [38]) Measurements of the guinea pig cochlea, for example, show that above a sound pressure level of about $20 \mathrm{~dB}$ the rate of response decreases down to $0.12 \mathrm{~dB} / \mathrm{dB}$ (cf. [45, 46, 22, 34]). Therefore, it becomes evident that the mechanics of the cochlea must be affected by some nonlinearities. Of course, such a nonlinear phenomena can not be reflected by the application of a linear cochlea model as used in this approach. Figure 4.4)(a) illustrates the linearity of the computational model presented here. This nonlinear behavior can be (partially) taken into account by the implementation of the sigmoid-function (as already discussed in chapter 2.3.3) that describes the nonlinear relation between the outer hair cell force and the hair bundle deflection.

Active Cochlea Model So far, only the traveling wave in the passive cochlea model has been considered. But, such a passive behavior represents only the mechanics of a dead cochlea. The dynamics of the living ear are quite different to those of a postmortem cochlea. As already discussed, the computational model, presented here, provides the possibility to control the activity of the outer hair cell driven force by means of a parameter $0 \leq \lambda \leq 1$, as originally suggested by Mammano [28]. 


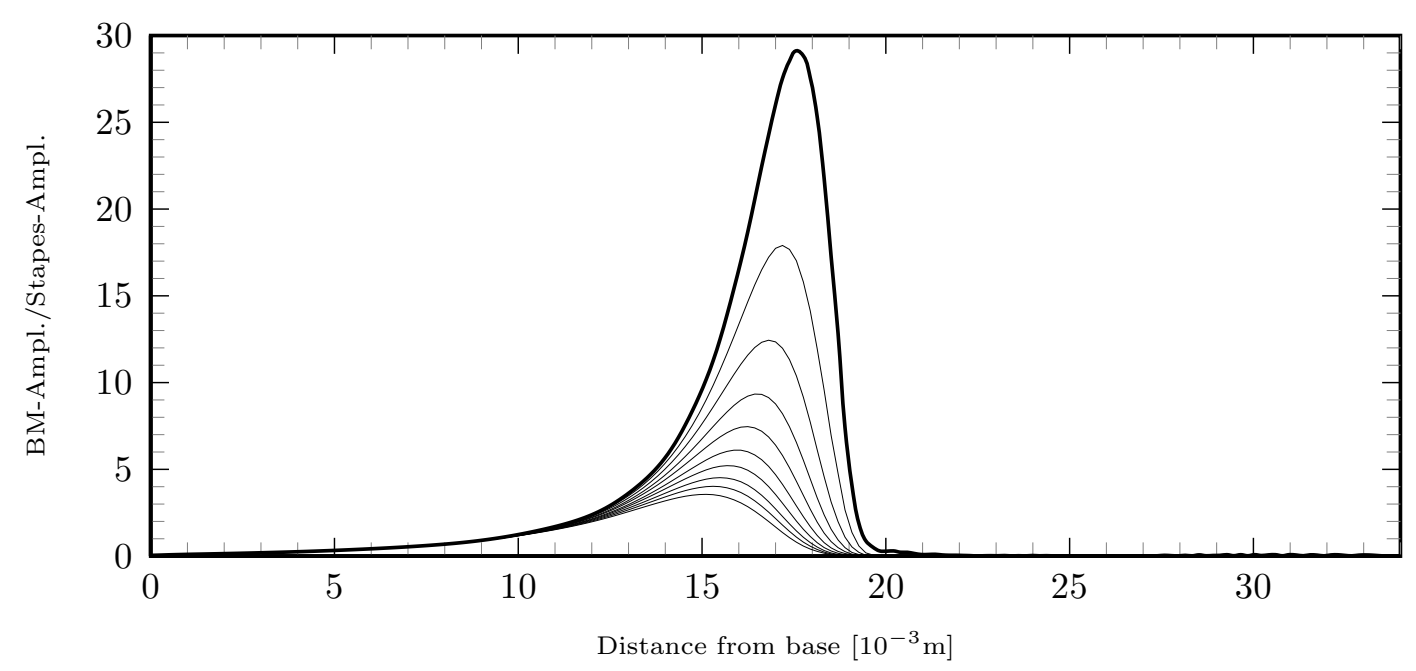

Figure 4.6: Envelopes of the traveling wave at a constant frequency of 1024Hz for a set of different outer hair cell activity parameters. The undermost line illustrates the envelope in the passive case $(\lambda=0.0)$ whereas the uppermost line is associated with the active cochlea model $(\lambda=0.9)$. It can be seen that the incremental increase of the activity parameter by a value of 0.1 is accompanied with a successive amplification of the envelope.

In principle, this approach is based on the local compensation of the positional viscosity of the basilar membrane. If $\lambda$ is set to zero, no outer hair cell driven force will act on the basilar membrane. This case describes the passive mechanics of the cochlea as already analyzed above. Otherwise, the fluid viscosity can be almost completely canceled, by increasing the outer-hair-cell activity parameter.

Figure 4.6 shows the resultant envelopes of the traveling waves at different outer hair cell activity parameters. In order to ensure the stability of the oscillations of the cochlear partition, the upper limit of the activity parameter is set to 0.9. Thus, the resultant net damping (which takes the cancelation of the outer hair cell motility into account) is at least $10 \%$ of the original damping that arises from the positional viscosity of a radial segment.

First of all, it should be noted that the amplification of the traveling wave motion is accompanied by a spatial shift of the peak in the direction of the apex. Such a shift is mostly consistent with experimental studies. It is an indication that the energy of the traveling wave motion is enhanced by the outer hair cells. (cf. [38]) Therefore, the characteristic places are closer to the functional relationship from Greenwood than in the passive case (cf. figure 4.5).

It can be clearly seen that the maximal displacement of the basilar membrane increases in dependence of the outer hair cell activity parameter up to the tenfold of the maximal displacement in the passive case. In literature different definitions of the cochlear amplifier gain are used (cf. [38]). In this paper, the gain is understood as the difference between the amplitudes of the active and the passive cochlea at the same locations on the basilar membrane. Within the cochlea of the guinea pig, gains between $65 \mathrm{~dB}$ and $78 \mathrm{~dB}$ were measured at the characteristic places of frequencies between $17 \mathrm{kHz}$ and $19 \mathrm{kHz}(\mathrm{cf}$. [45, 


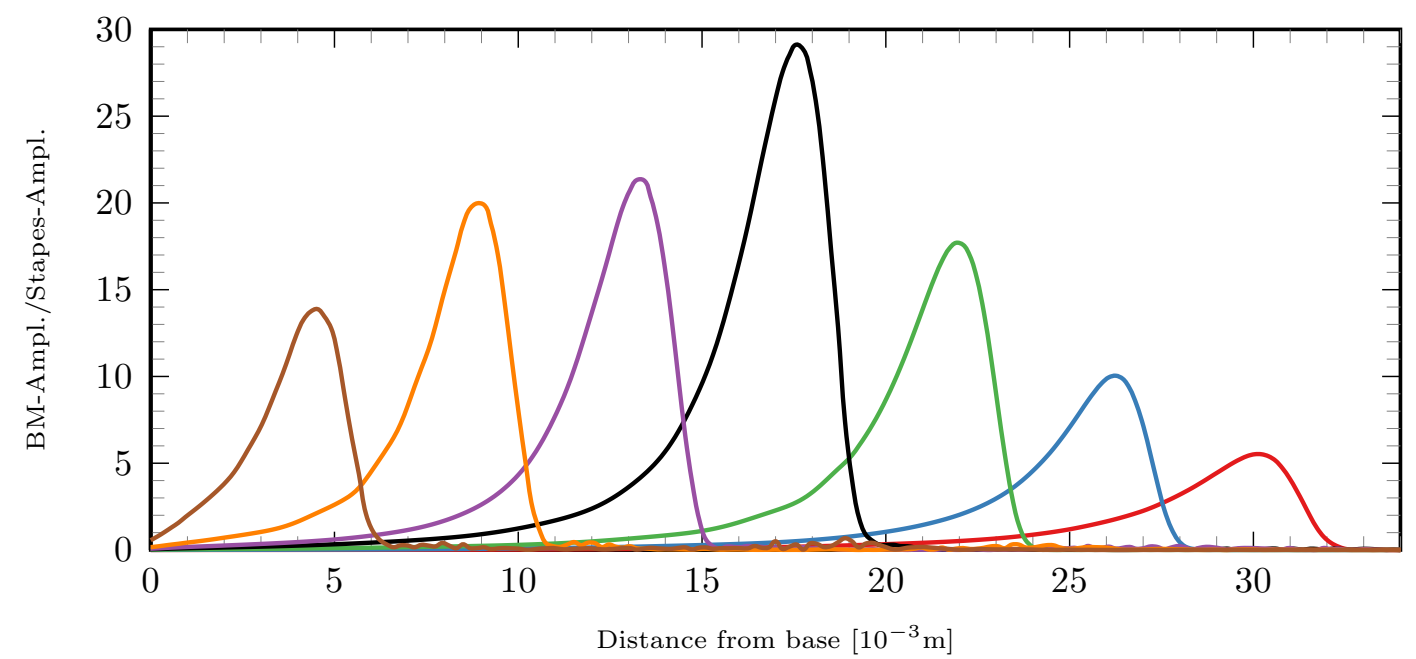

(a)

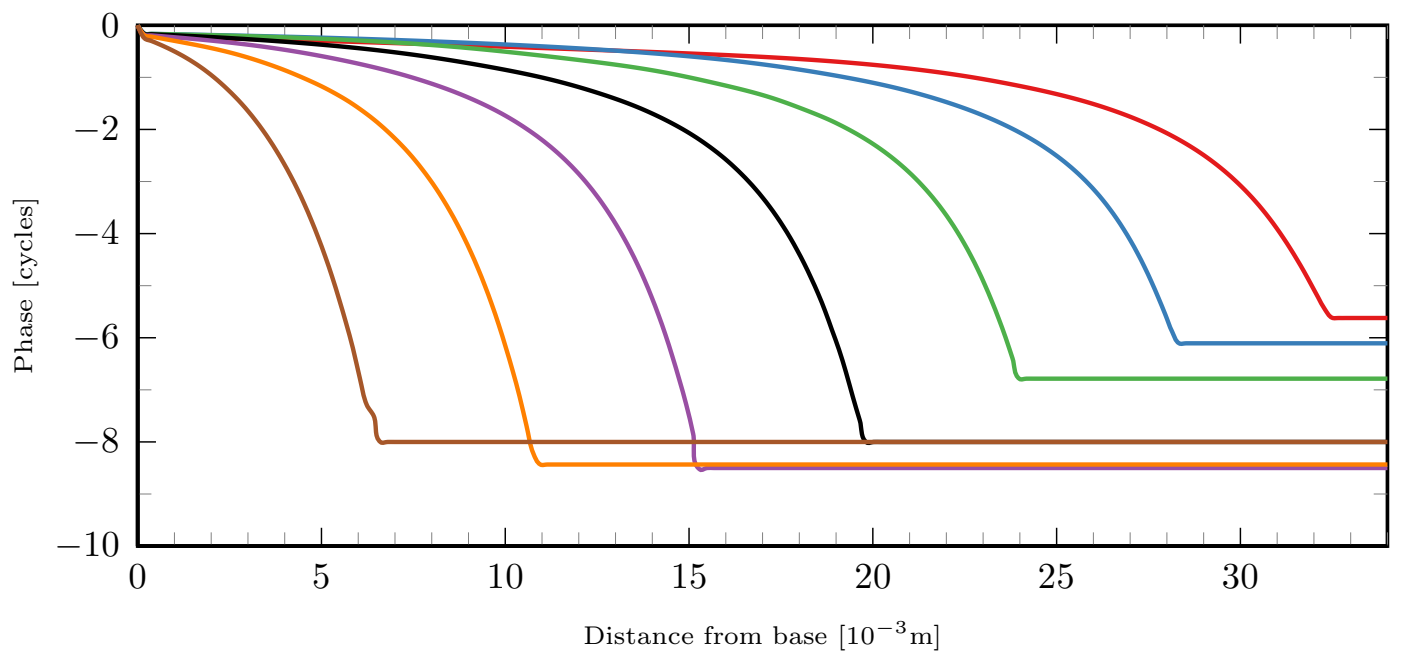

(b)

Figure 4.7: Envelopes (a) and phases (b) of the traveling wave in the active cochlea model computed at constant stape displacement for a set of different frequencies: $128 \mathrm{~Hz}$ (red line), $256 \mathrm{~Hz}$ (blue line), 512 Hz (green line), 1024Hz (black line), 2048Hz (purple line), 4096Hz (orange line), 8192Hz (brown line). 


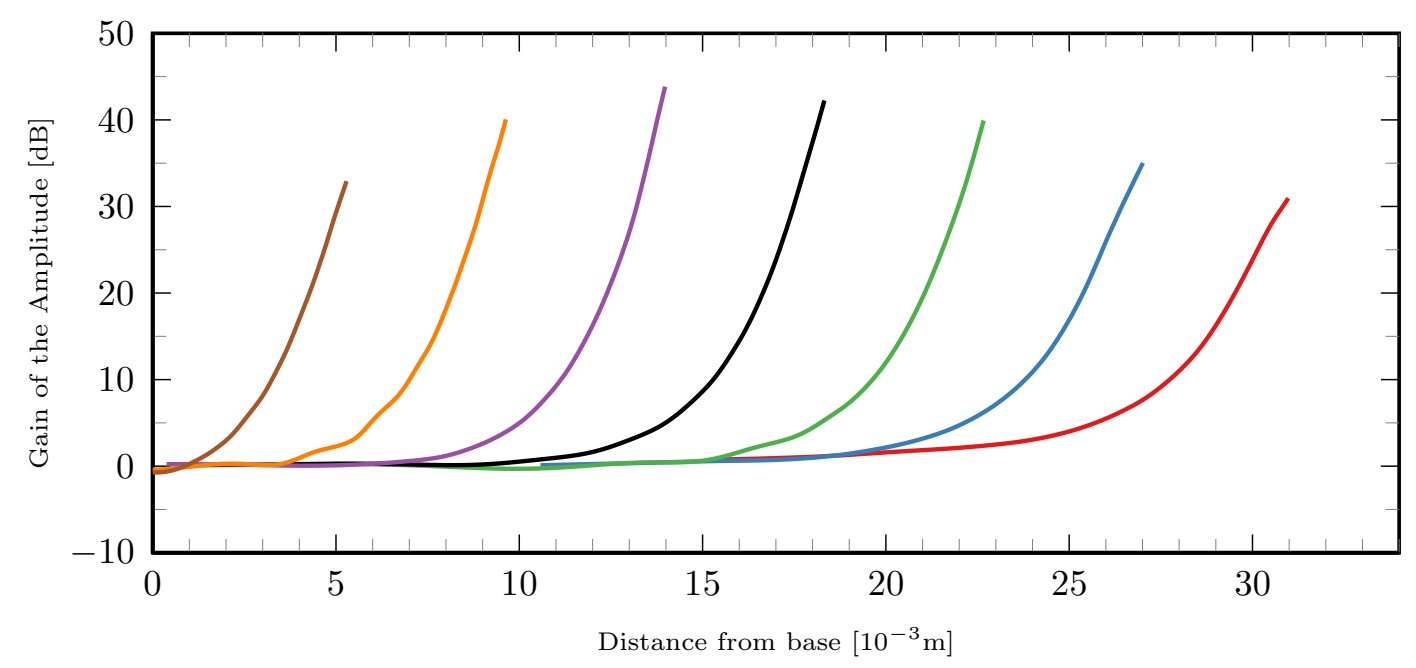

Figure 4.8: Positional gain of the envelopes at different frequencies in the active cochlea model $(\lambda=0.9)$ with respect to the envelopes in the passive cochlea model $(\lambda=0.0)$ : $128 \mathrm{~Hz}$ (red line), $256 \mathrm{~Hz}$ (blue line), $512 \mathrm{~Hz}$ (green line), $1024 \mathrm{~Hz}$ (black line), $2048 \mathrm{~Hz}$ (purple line), 4096Hz (orange line), 8192Hz (brown line).

46, 22, 34]). As shown in figure 4.8, such a gain of the amplitude can not be completely reproduced by the computational model presented in this work. Nevertheless, the model presented in this work provides an instrument to investigate the effects of the amplification mechanisms on the resultant acoustically driven flows. On the basis of these results, further conclusions may be drawn with regard to the impact of acoustic streaming at even higher gains.

In addition, the traveling wave is characterized by an higher phase lag than inthe passive cochlea model (cf. figure 4.7 (b)] . In consideration of the envelopes (cf. figure 4.7 (a) it can be seen that the respective shapes in the active model are significantly sharper than in the passive case. This property of the amplification mechanism is believed to be a very important aspect with respect to the acoustic perception, because it enhances the frequency selectivity.

\subsubsection{Motions within the Stokes Boundary Layer}

Because the dissipation of acoustic energy within the thin Stokes boundary layer next to the basilar membrane is a major cause for second order flows, particular attention has to be paid on an accurate numerical approximation of the motions within these boundary layers. The results of the computational model presented in this work can be validated by comparing the numerically determined motions with some theoretical considerations from Lighthill [25]. In his very important contribution, Lighthill estimated the dimension of acoustic streaming within the inner ear on the basis of a mathematical description of the flow field adjacent to the basilar membrane. In this context, he suggested to approximate the three-dimensional distribution of the fluid motion next to the traveling wave of the 


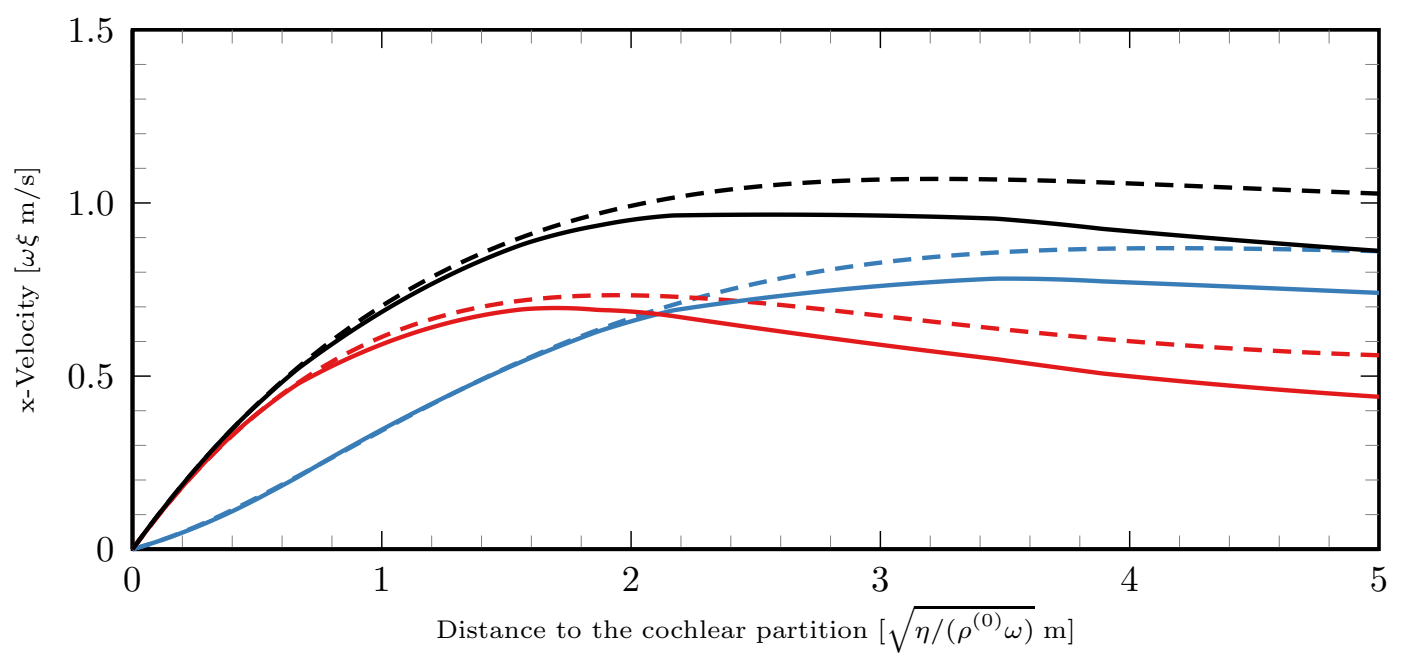

(a)

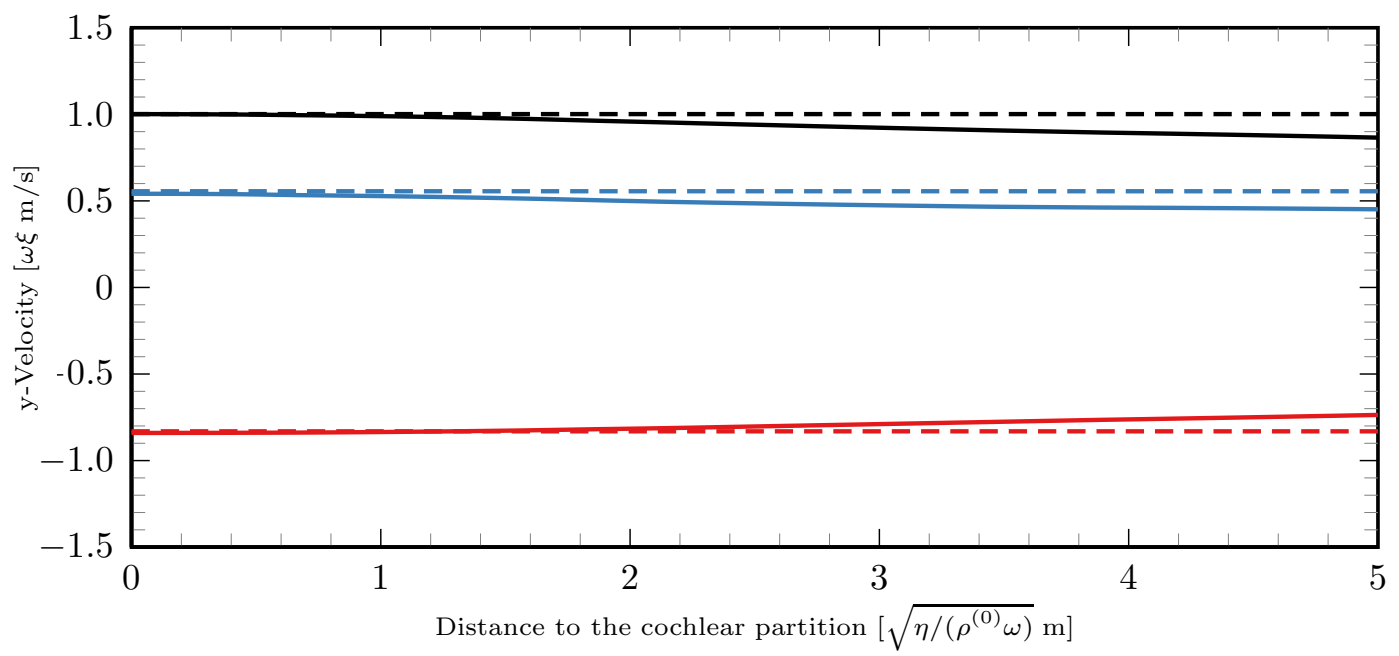

(b)

Figure 4.9: Comparison between the numerical results of the computational model (solid lines) presented in this work and the theoretical approximation (dashed lines) of the fluid motions within the Stokes boundary layer from Lighthill [25] near to the characteristic place at a stimulation frequency of 1024Hz in the passive model. Figure (a) illustrates the longitudinal velocity components whereas (b) shows the vertical components. The red, blue and black lines are associated with the the cosine-part, the sine-part and the amplitude of the respective velocity component. 
basilar membrane by the superposition of solutions of the Laplace equation

$$
\frac{\partial^{2} \phi}{\partial x_{2}^{2}}+\frac{\partial^{2} \phi}{\partial x_{3}^{2}}-k^{2} \phi=0
$$

where $\phi$ denotes the velocity potential. By the use of the so-called WKB method (also known as Liouville-Green method), Lighthill reasoned that the fluid motion outside of a thin boundary layer of the basilar membrane near to the characteristic place can be mainly approximated by a two-dimensional circular motion perpendicular to the radial axis. In terms of a mathematical formulation, this motion can be written as

$$
\begin{aligned}
& v_{1}=\frac{\partial \phi}{\partial x_{1}}=\mathrm{i} \xi \mathrm{e}^{-k x_{2}} \\
& v_{2}=\frac{\partial \phi}{\partial x_{2}}=\xi \mathrm{e}^{-k x_{2}} .
\end{aligned}
$$

But in order to meet the no-slip boundary conditions at the basilar membrane, equation 4.3 and 4.4 have to be adapted according to Rayleigh's law of streaming [35], yielding

$$
\begin{aligned}
& v_{1}=\mathrm{i} \xi\left(1-\mathrm{e}^{-x_{2} \sqrt{\mathrm{i} \omega \rho / \eta}}\right), \\
& v_{2}=\xi\left(1+\left(1-\mathrm{e}^{-x_{2} \sqrt{\mathrm{i} \omega \rho / \eta}}\right) \frac{\omega}{c} \sqrt{\frac{\eta}{\mathrm{i} \omega \rho}}\right) .
\end{aligned}
$$

Figure 4.9 shows the numerically determined motions within the thin boundary layer in comparison to the analytical results from Lighthill. The thickness of the boundary layer can be approximately determined to $5 \sqrt{\eta / \rho \omega}$. With regard to typical stimulation frequencies and the spatial dimensions of the cochlea, the Stokes boundary layer is comparatively small. Therefore, it is very important that the finite element discretization process constructs a mesh that is sufficiently small to ensure an adequate approximation of the velocity and pressure field. It can be noted that the motions close to the boundary are nearly identical. The greater the distance to the cochlear partition, the greater the deviation of the numerical results from the analytical approximations.

In consideration of the differences between both approaches, these deviations between the results seem to be relatively small. In contrast to the computational model presented here, Lighthill's model neglected the energy dissipation due to viscous forces within the bulk as well as some interfering effects arising from the interaction with the solid boundaries. In addition to this, by the use of the Laplace equation 4.2 it is assumed that the motions can be described as an irrotational and incompressible flow. Otherwise, also the numerical evaluation has the well-known limitations in accuracy, based on the local resolution of the mesh, the choice of form-functions as well as other numerical effects. Whilst taking all these aspects into account, the computational model yields very good results when comparing the numerical results of the motion within the Stokes boundary layer with the analytical approximations from Lighthill. 


\subsection{Fluid Flows driven by the Second Order System}

\subsubsection{Difference between Eulerian and Lagrangian Mean Motions}

As already mentioned in chapter 2.4.1, a distinction between the Eulerian and the Lagrangian specification of the fluid-motion is essential for studying acoustically driven flows. With respect to the first order system, such a distinction is not necessary since both specifications lead to equal results in the case of the equilibrium state. But with regard to the second order system a difference between both descriptions arises through the nonlinear terms.

The distinction between both concepts has already been considered in the context of the derivation of the second order boundary condition 2.52. The same approach can also be used to describe the difference of both velocity specifications within the bulk of the fluid. At the boundaries the Lagrangian velocity vanishes due to the no-slip condition. But if the Lagrangian second order mean velocity $\boldsymbol{v}^{(\mathcal{L})}$ is taken into account, the difference can be evaluated to

$$
\boldsymbol{v}^{(d c)}-\boldsymbol{v}^{(\mathcal{L})}=-\frac{1}{\rho^{(0)} c_{0}^{2}}\left\langle p^{(1)} \boldsymbol{v}^{(1)}\right\rangle+\frac{1}{2} \operatorname{rot}\left\langle\boldsymbol{\zeta}^{(1)} \times \boldsymbol{v}^{(1)}\right\rangle
$$

where $\boldsymbol{\zeta}^{(1)}$ denotes the first order displacement of the fluid particle (cf. [9, 26]). The analysis of the resultant velocities, calculated by the computational model, has revealed that this difference plays only a subordinate role in the context of acoustic streaming within the bulk of the inner ear.

With respect to the boundary condition at the cochlear partition, it becomes apparent that the first summand on the right hand side of equation 4.7 can be associated with a vertical inflow or outflow. In consideration of the specific assumptions made by the modeling process, the second term describes, in contrast, a slipping flow. Both the resultant slipping flows and the vertical flows are illustrated in figure 4.10, It can be noticed that the amount of the slipping velocity exceeds the amount of the vertical velocities by several orders of magnitude.

The Eulerian slipping flow at the basilar membrane was also taken into account in the work from Lighthill [25]. On the basis of the first order velocity components 4.5] and 4.6, Lighthill calculated the second order mean velocity at the cochlear partition as

$$
v_{1}^{(d c)}=\sqrt{\frac{\rho}{\mu \pi f}} \frac{\left(v_{2}^{(\mathrm{amp})}\right)^{2}}{4} .
$$

It has been revealed that the slipping flow calculated by the computational model slightly exceeds the theoretical estimates from Lighthill (cf. figure 4.10). 


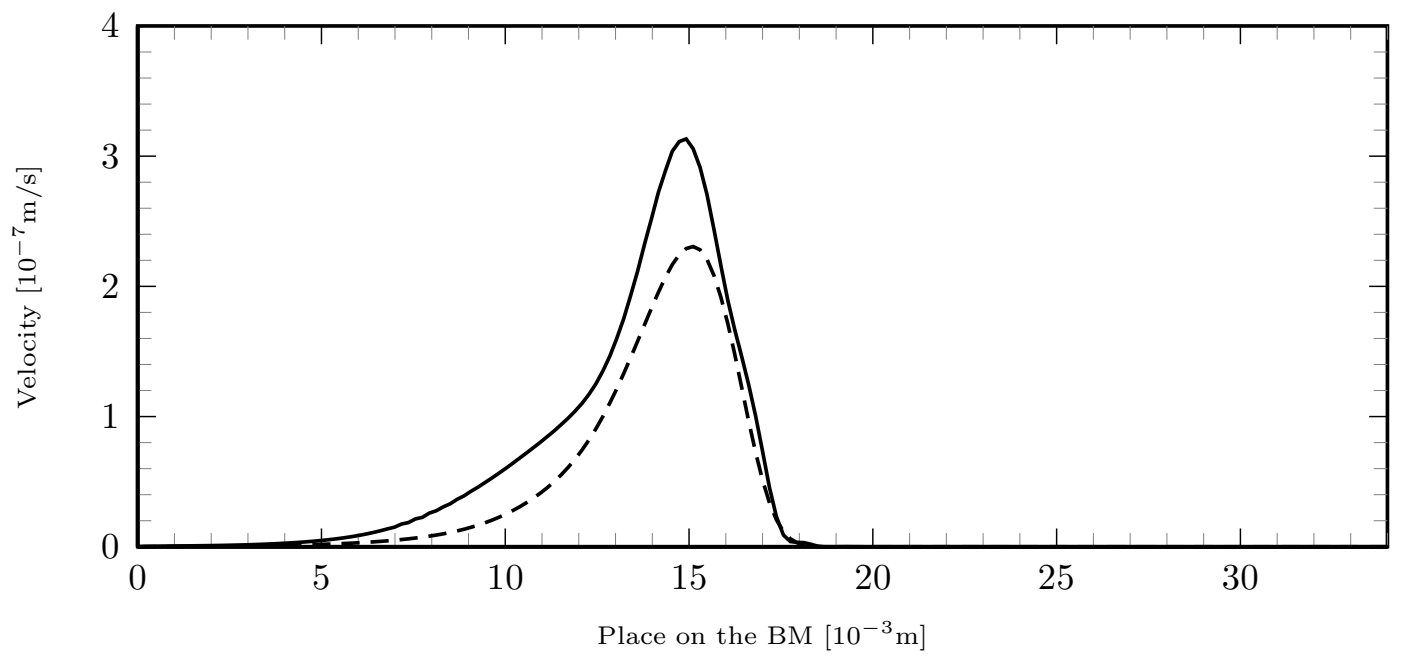

(a)

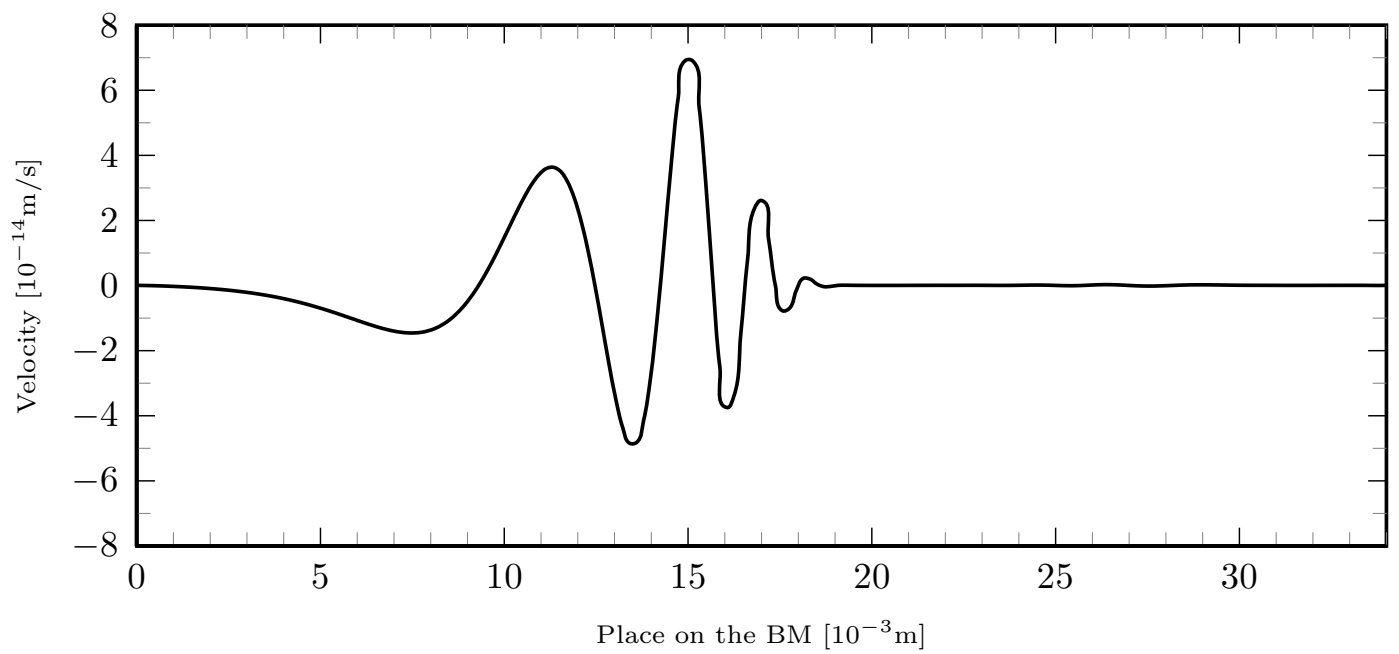

(b)

Figure 4.10: Difference between the Lagrangian and the Eulerian second order mean velocity at the cochlear partition. Due to the no-slip condition this difference coincides with the boundary condition of the acoustic streaming subproblem. The x-component of this difference is associated with a slipping flow at the basilar membrane. Figure (a) compares the numerically determined slip velocity (solid line) with the theoretical estimate of the flow velocity (dashed line) from Lighthill [25] at a stimulation frequency of $1024 \mathrm{~Hz}$ in the passive cochlea model. Figure (b) illustrates the $x_{2}$-component of this difference which corresponds to a vertical flow. 


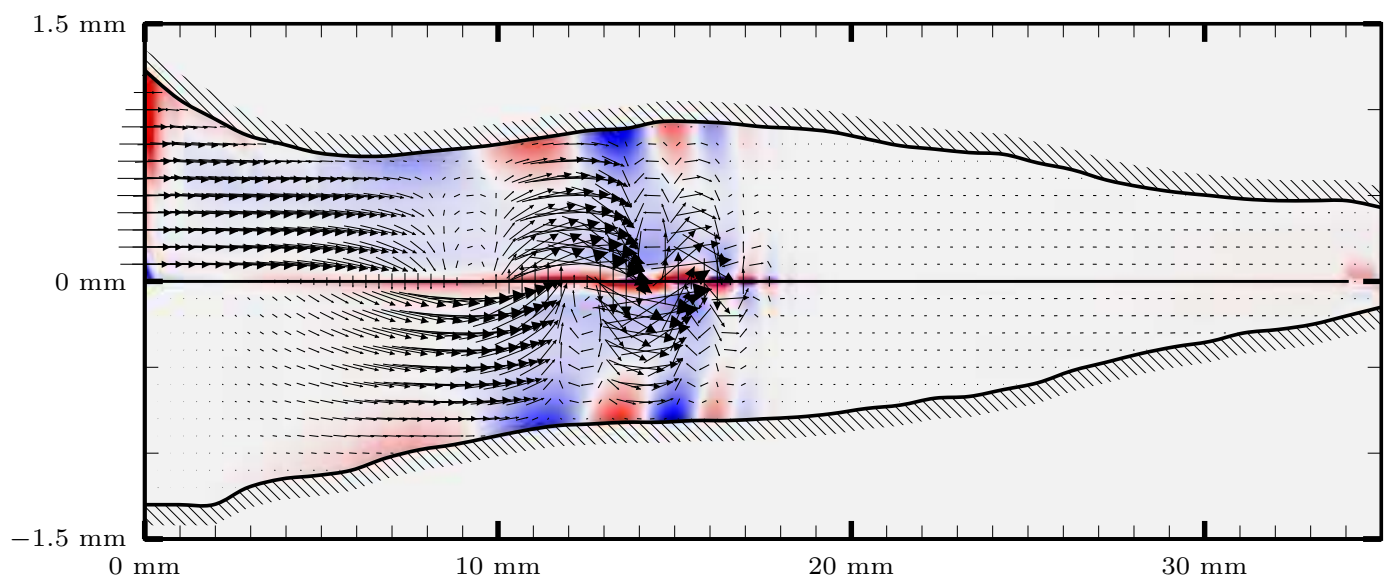

(a)

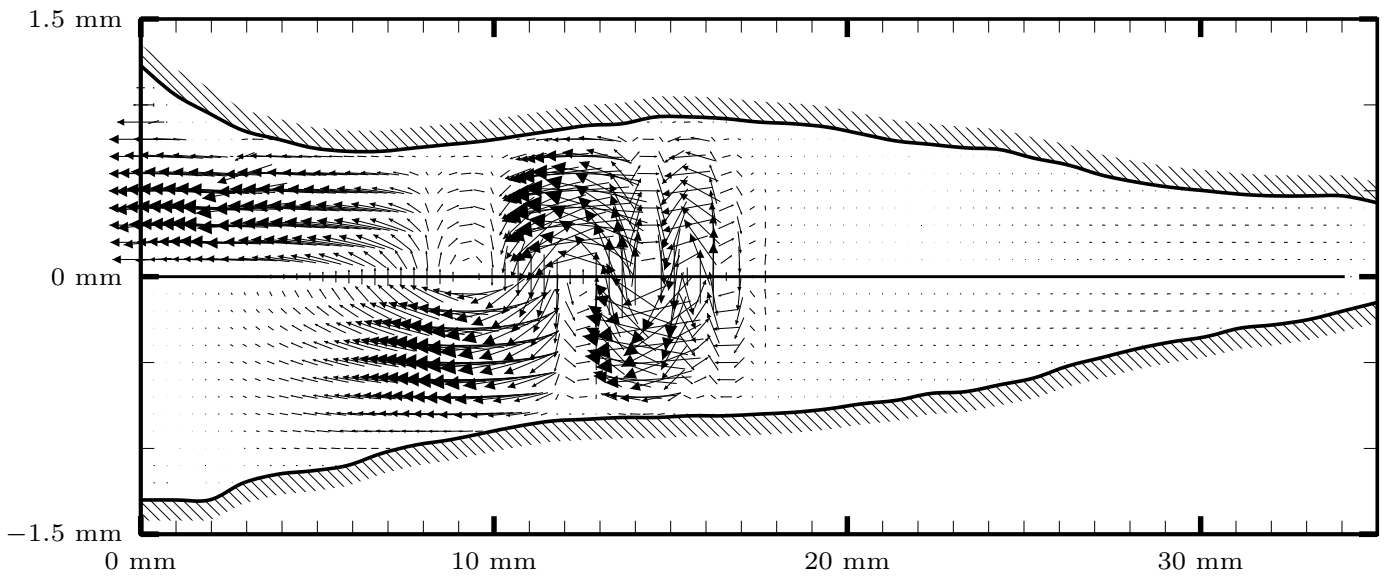

(b)

Figure 4.11: The vector field of figure (a) illustrates the mass that is transported by the acoustic intensity field. In the red colored regions the acoustic intensity field weakens and therefore these areas act as a mass source. In contrast to that, the blue colored region acts as a mass sink since the acoustic intensity field become more intense. Figure (b) shows the resultant second order mean flow caused by the mass sources and mass sinks of the first order system. 


\subsubsection{Mass Source driven Streaming}

According to Bradley [9], the mean mass of second order that is transported within the fluid can be written as

$$
\begin{aligned}
\left\langle\boldsymbol{J}^{(2)}\right\rangle: & =\rho^{(0)} \boldsymbol{v}^{(d c)}+\left\langle\rho^{(1)} \boldsymbol{v}^{(1)}\right\rangle \\
& =\rho^{(0)} \boldsymbol{v}^{(d c)}+\frac{1}{c_{0}^{2}}\langle\boldsymbol{I}\rangle
\end{aligned}
$$

where $\boldsymbol{I}:=p^{(1)} \boldsymbol{v}^{(1)}$ denotes the acoustic intensity. It can be seen that mass can be transported either by the acoustic streaming flow field (first term) or by the acoustic intensity field (second term). The velocity of the acoustic intensity driven mass transport, given by $\frac{1}{\rho^{(0)}}\langle\boldsymbol{I}\rangle$, is shown in figure 4.11 (a). This kind of mass transport is not associated with a real existing flow. It occurs if the fluid is on average more dense when the direction of the fluid motion corresponds to the orientation of the mass current than at the time when the fluid moves in the opposite direction. In such a case it is quite obvious that the transport of mass is not balanced over one period and a net mass current appears.

By integrating the intensity driven mass transport over the closed surface $S=\delta V$ of an arbitrarily chosen volume $V$ and by applying the divergence theorem, the relation

$$
\frac{1}{c_{0}^{2}} \int_{\delta V}\langle\boldsymbol{I}\rangle \mathrm{d} S=\int_{V} \tilde{\boldsymbol{m}} \mathrm{d} V
$$

holds. Therefore, it becomes apparent that the acoustic intensity driven mass transport is closely related to the mass source distribution $\tilde{\boldsymbol{m}}$ which appears in the second order mass equation 2.46. It can be concluded that the net inward flux of the intensity driven mass transport field through a closed surface coincides with the amount of mass that is released within the enclosed region by the mass source term $\tilde{\boldsymbol{m}}$. Equation 4.10 is valid for an arbitrarily chosen volume. Therefore, mass sources can occur in all regions where the acoustic intensity weakens. In contrast, regions can also act as a sink if the acoustic intensity field strengthens. (cf. [9])

As already discussed in section 4.2.1, also the oscillating boundary structures are capable of acting as a source or as a sink of mass. If the volume $V$ in equation 4.10 is chosen to be identical to the whole fluid-volume of the cochlear enclosure it can be seen that the amount of mass taken up by the basilar membrane and the oval window is equal to the amount of mass that is injected by the mass source distribution.

The acoustically driven flows induced by the mass transport mechanism are illustrated in figure 4.11 (b). But the numerical results show that the velocities of the resultant acoustic streaming flow field caused by the mass transport mechanism are negligible in comparison to the other mechanisms. 


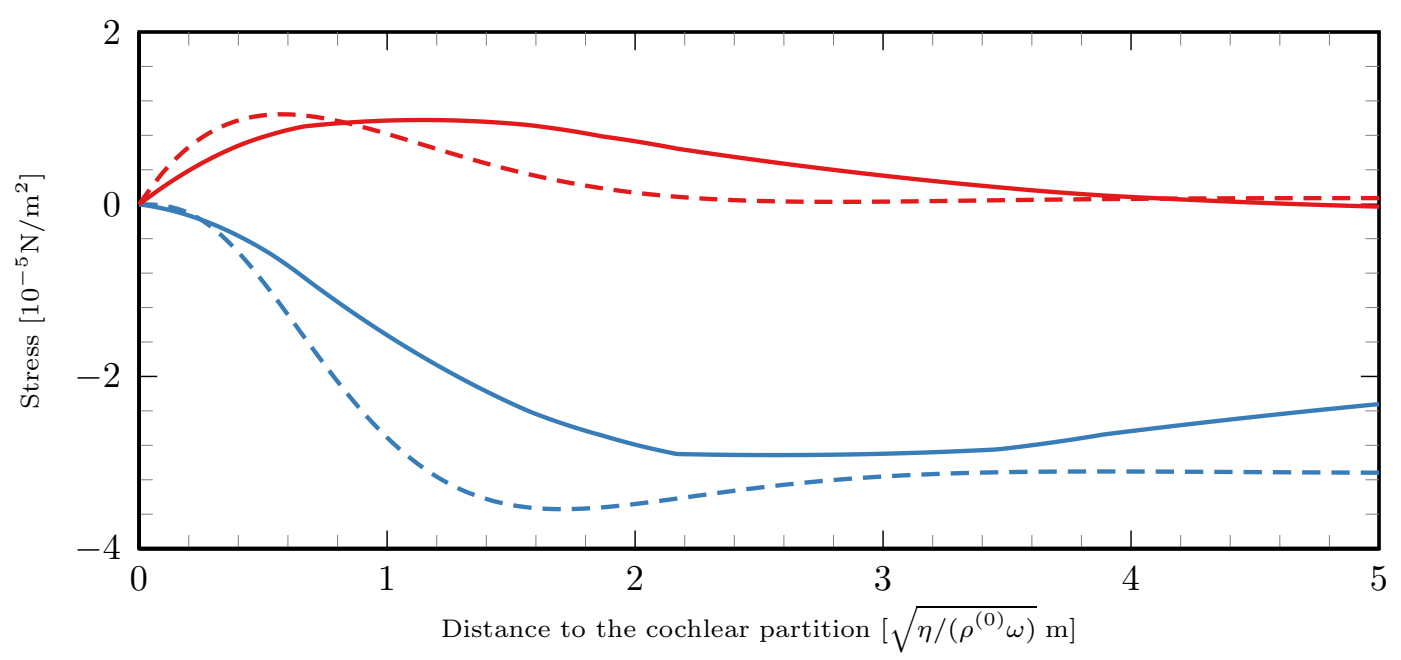

Figure 4.12: The graph illustrates the resultant Reynolds stresses of the computational model (solid lines) within in the Stokes boundary layer in comparison to theoretical approximations (dashed lines) from Lighthill [25]. The data originates from the characteristic place at a stimulation frequency of 1024Hz with respect to the passive cochlea model. The red lines depict the shear stress component that describes the $x_{2}$-flux of $x_{1}$-momentum per unit area. In contrast, the blue lines are associated with the normal stress component that represents the $x_{1}$-flux of $x_{1}$-momentum per unit area.

\subsubsection{Force Source Driven Streaming}

In this section, the influence of the force-source term 2.49 on the capability to generate significant mean motions is analyzed. In order to understand the physical origin of the resulting forces, Bradley [9] pointed out that the force-source distribution, accurate to the second order, can be written as the product of the fluid-acceleration $\boldsymbol{a}=\frac{\partial \boldsymbol{v}}{\partial t}+(\operatorname{grad} \boldsymbol{v}) \boldsymbol{v}$ and the density:

$$
\tilde{\boldsymbol{f}}=-\langle(\rho \boldsymbol{a})\rangle+\mathcal{O}\left(\epsilon^{3}\right) .
$$

Therefore, the exerted force depends mainly on the phase between the fluid acceleration and the density. The force $\tilde{\boldsymbol{f}}$ can also be represented in terms of the well-known Reynolds stress tensor which can be expressed as $\rho v_{i} v_{j}$. By using integration by parts, the (first order) mass conservation 2.22 and the product rule, the force source distribution can be written as the spatial variation of the Reynolds stress:

$$
\tilde{\boldsymbol{f}}=-\rho^{(0)}\left\langle\frac{\partial v_{i}^{(1)} v_{j}^{(1)}}{\partial x_{i}}\right\rangle \boldsymbol{e}_{j} .
$$

This force can furthermore be decomposed into a shear stress component and a normal stress component. The shear stress components describe the flux of momentum per unit area perpendicular to its orientation whereas the normal stress components are characterized by equal directions of the force and its flux.

The results of the experiments, performed by the computational model, show that the 


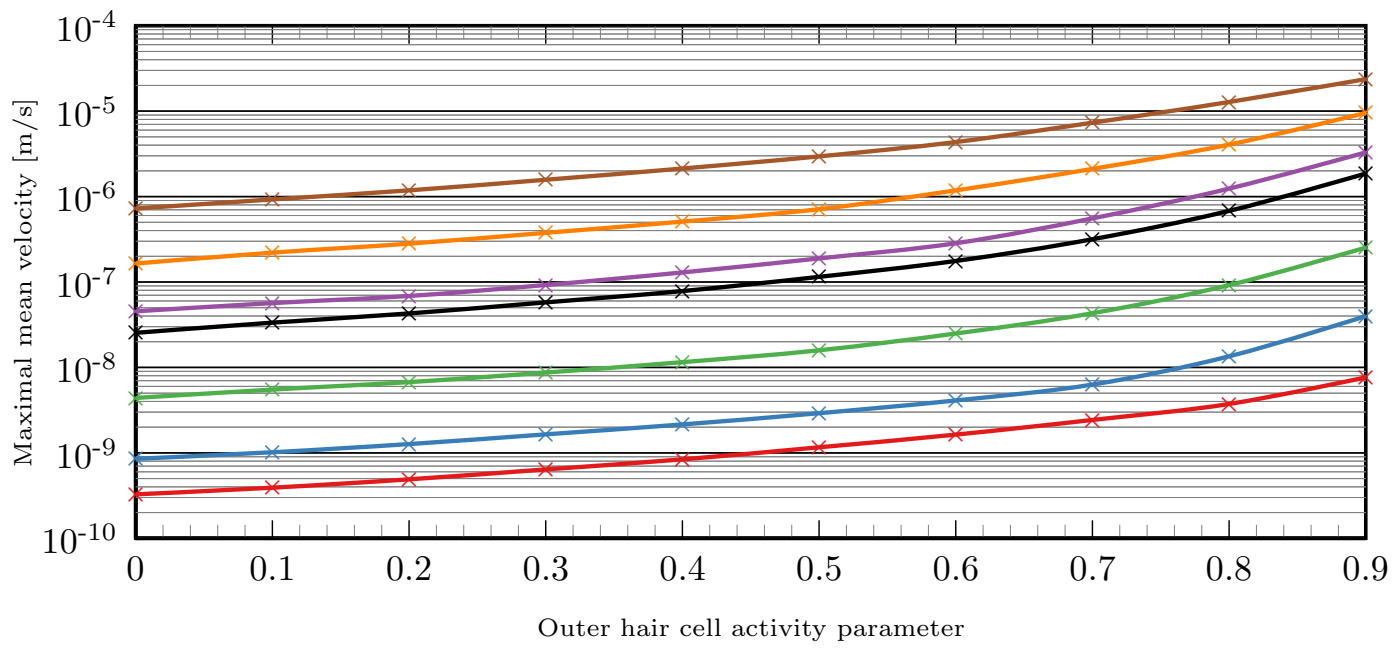

(a)

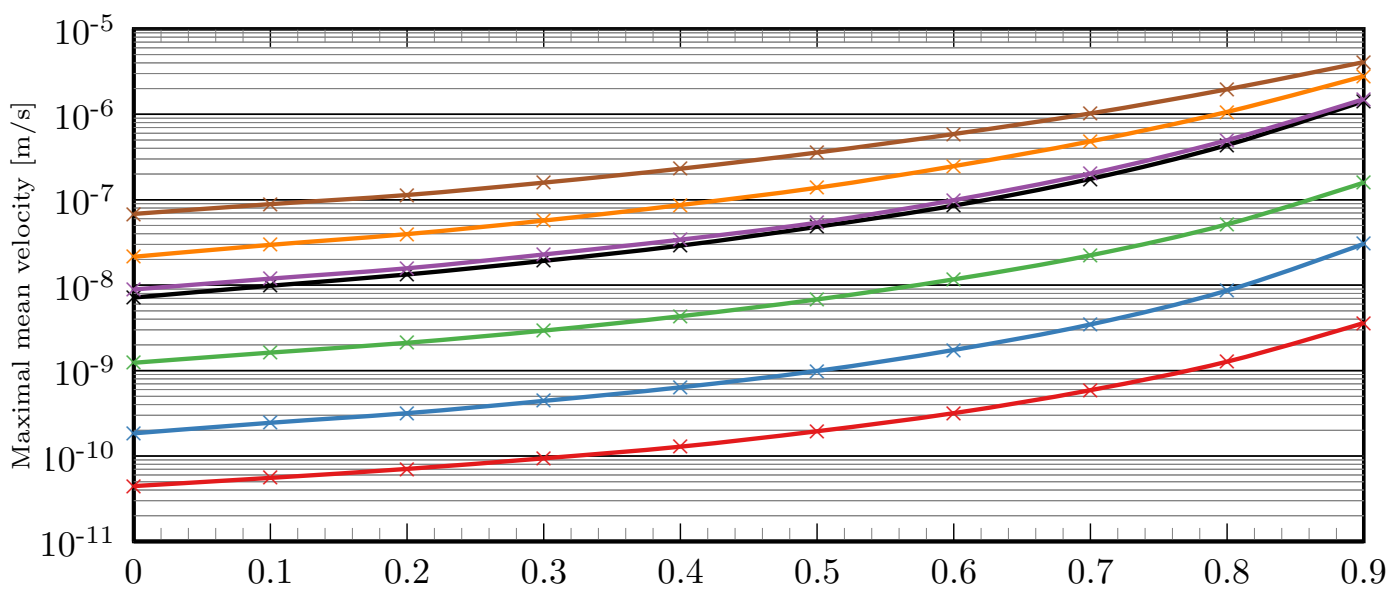

Outer hair cell activity parameter

(b)

Figure 4.13: Figure (a) shows the Eulerian specification of the maximal mean velocity outside the Stokes boundary layer that are determined by the computational model. By comparison, figure (b) illustrates Lighthill's estimate (cf. [25]) of the maximal mean velocity. The velocities are calculated on the basis of the numerical results of the first order system at different frequencies and outer hair cell activities: 128Hz (red line), $256 \mathrm{~Hz}$ (blue line), 512Hz (green line), 1024 Hz (black line), $2048 \mathrm{~Hz}$ (purple line), 4096Hz (orange line), 8192Hz (brown line). 
forces are predominant at the adjacent boundary layers of the basilar membrane near to the characteristic place. This observation is in conformity with the theoretical considerations from Lighthill [25] whose estimation of acoustic streaming is mainly based on the Reynolds stresses occurring within the Stokes boundary layer. In his work he evaluated an effective slip velocity outside of the thin Stokes boundary layer. In terms of the velocity amplitude of the oscillating basilar membrane, this effective slip flow can be expressed as

$$
\frac{\pi f k}{2}\left(\xi^{(\mathrm{amp})}\right)^{2}-\frac{3 \pi f}{2} \xi^{(\mathrm{amp})} \frac{\partial \xi^{(\mathrm{amp})}}{\partial x_{1}} .
$$

The first term as well as one third of the second term originate from the shear stress component that transports the $x_{1}$-momentum in the $x_{2}$-direction. In contrast, two thirds of the second summand can be attributed to the normal component of the Reynolds stress driven force. A detailed derivation of this slipping flow estimate can be found in the work from Lighthill [25].

Figure 4.12 shows the numerically determined stresses in comparison to the stresses that are calculated on the basis of the velocity components 4.5 and 4.6 used by Lighthill. The small deviations between both models can presumably be explained by previous differences regarding the fluid velocity (cf. figure 4.9) and the boundary condition (cf. figure 4.10 (a) as well as the differences with respect to the underlying assumptions at the development of both models. Due to the very small dimensions of the Stokes boundary layer, numerical errors must also be considered as a possible cause for this deviation.

The formula from Lighthill can be used to calculate the effective slip velocity of acoustic streaming on the basis of the first order field resulting from the computational model. The resultant maximal velocities are shown in figure 4.13(b). In turn, figure 4.13 (a) illustrates the maximal mean velocities outside of the Stokes boundary layer that are calculated on the basis of the computational model. By comparing both results it can be noticed that the velocities of the computational model are up to one order of magnitude larger than Lighthill's estimates.

\subsubsection{Békésy's eddies}

The numerical simulation of the computational model has shown that acoustic streaming is mainly driven by boundary layer mechanisms (cf. chapter 4.2.3). Acoustically driven flows that are based on mass-transport mechanisms (cf. chapter 4.2.2) are not as significant as the previously mentioned phenomenon. At a specific frequency, the mean fluid motion of second order can be best described by two eddies, which are almost symmetrically arranged to the cochlear partition. Of course, this circular movement must be thought of as superimposed by the first order fluctuations. But a separated evaluation is useful, since both motions can be described on different time-lines due to their difference between both velocities. The direction of the rotation of each eddy is specified by an apical directed motion of the particles that are located next to the partition. Thus, an opposite directed flow occurs close to the outer boundary. The velocity of each eddy varies along its streamlines. 


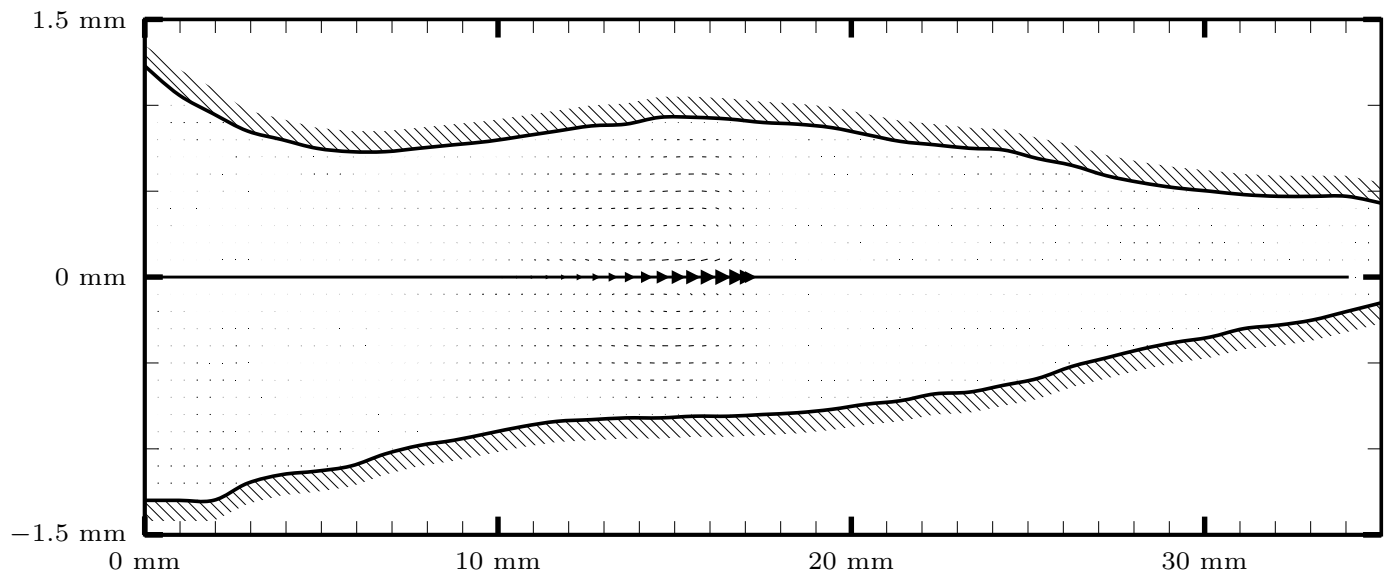

(a)

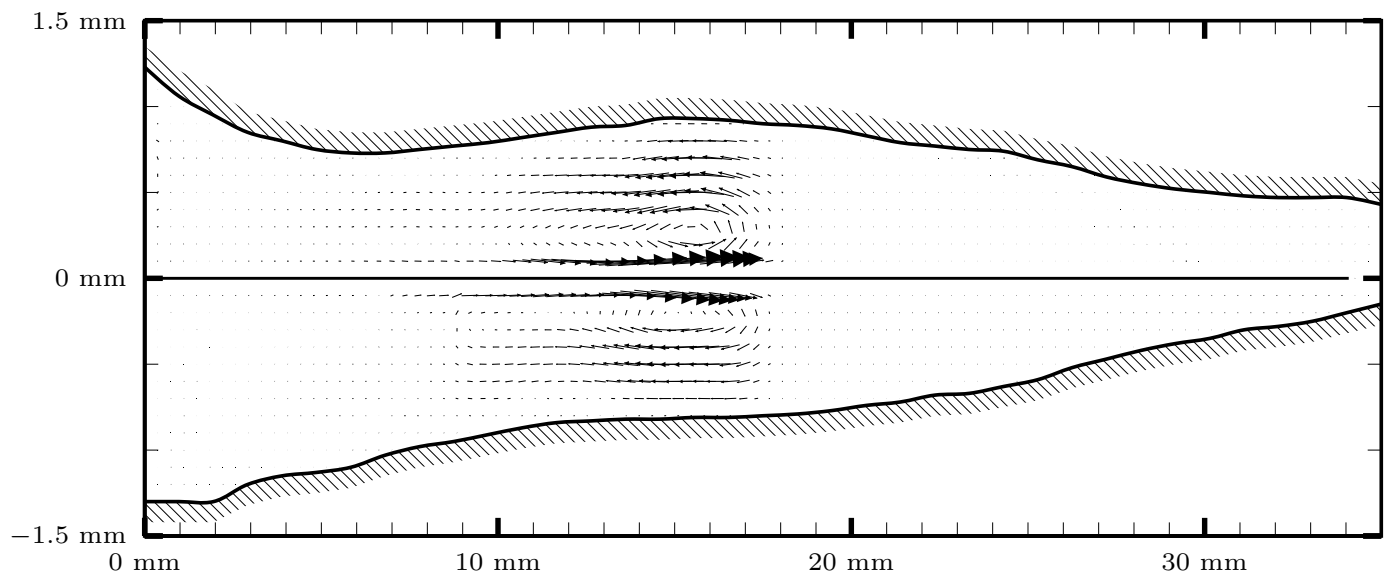

(b)

Figure 4.14: The Eulerian (a) and the Lagrangian (b) mean velocity field of the second order system at a stimulation frequency of $1024 \mathrm{~Hz}$ in the passive cochlea model. The flow field constitutes a first order approximation of acoustic streaming and is characterized by two eddies above and below the cochlear partition near to the characteristic place. It can be seen that the no-slip condition at the basilar membrane of the Lagrangian flow field is accompanied by an Eulerian slipping flow. It is important to note that both figures are not directly comparable with respect to the velocities represented by the arrow lengths, due to a different scaling of both vector fields that is oriented towards their respective maximal velocity. 


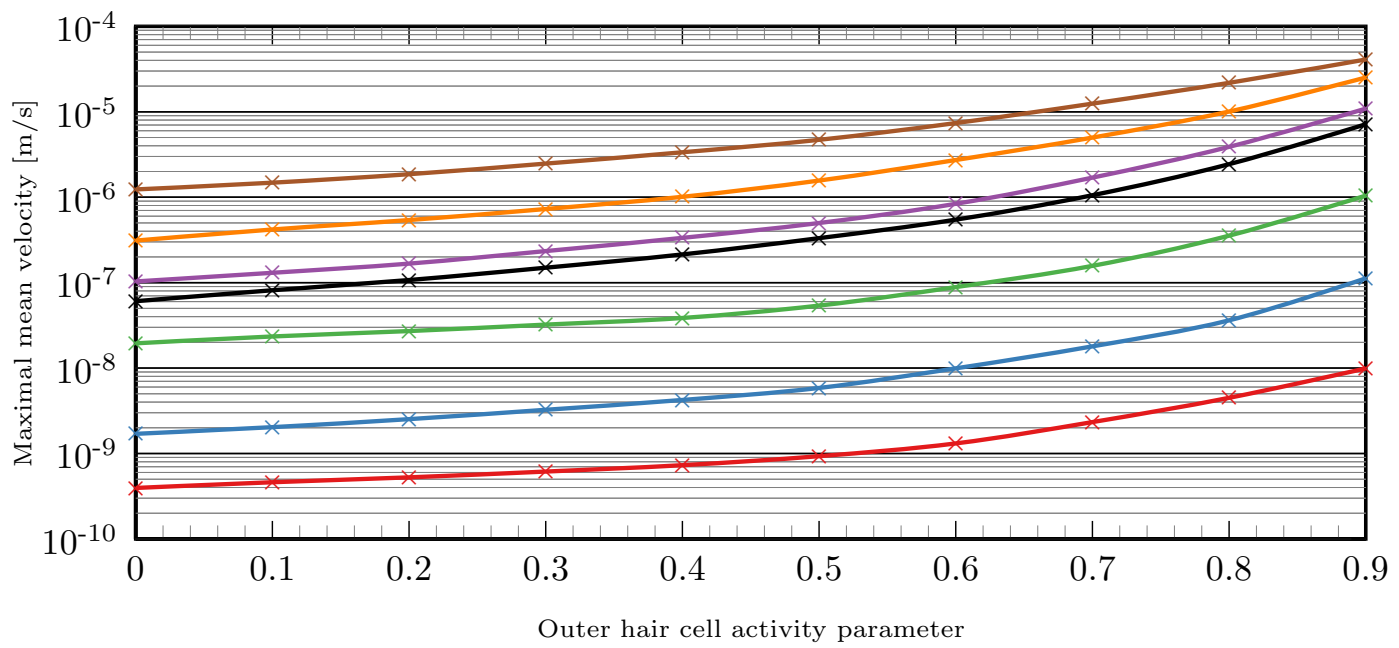

Figure 4.15: Lagrangian specification of the maximal mean velocity at different stimulation frequencies and outer hair cell activities: $128 \mathrm{~Hz}$ (red line), $256 \mathrm{~Hz}$ (blue line), 512Hz (green line), 1024 Hz (black line), $2048 \mathrm{~Hz}$ (purple line), $4096 \mathrm{~Hz}$ (orange line), 8192Hz (brown line).

Next to the cochlear partition, the fluid is accelerated in the direction of the apex until a point of maximal velocity is reached. Shortly behind the position of maximal velocity, the direction of the fluid motion turns back and the velocity decreases.

Dependency of the frequency The longitudinal position of the center of each eddy is nearly identical to the position of the maximal displacement of the basilar membrane.

Dependency of the amplitude As it can be seen in figure 4.16, the maximal velocity of the mean flow is proportional to the square of the stimulation amplitude. Such a quadratic dependence on the velocity was also observed by the experiments from Tonndorf [4]. In addition, the results indicate that the amplitude has no (significant) influence on the shape of the eddies. In other words, the change of the amplitude causes only a spatially uniform (scalar) scaling of the vector field that represents the mean flow.

Dependency of the outer hair cell amplification Figure 4.15 illustrates that the velocity of the acoustically driven flows grows almost exponentially with the outer hair cell activity parameter. But in contrast to the amplitude, this growth depends on various factors such as the gain of the displacement of the basilar membrane, the phase velocity of the traveling wave and its envelope. Furthermore, the activity parameter also influences the shape of the eddies. By comparing the eddies of the active model (cf. chapter A.4) with the passive model (cf. chapter B.3) it can be clearly seen that the increase of the activity parameter is accompanied with a decrease of the eddy size.

In summary, it can be noted that the numerical results are almost in conformity with the experimental studies from Tonndorf [48]. 


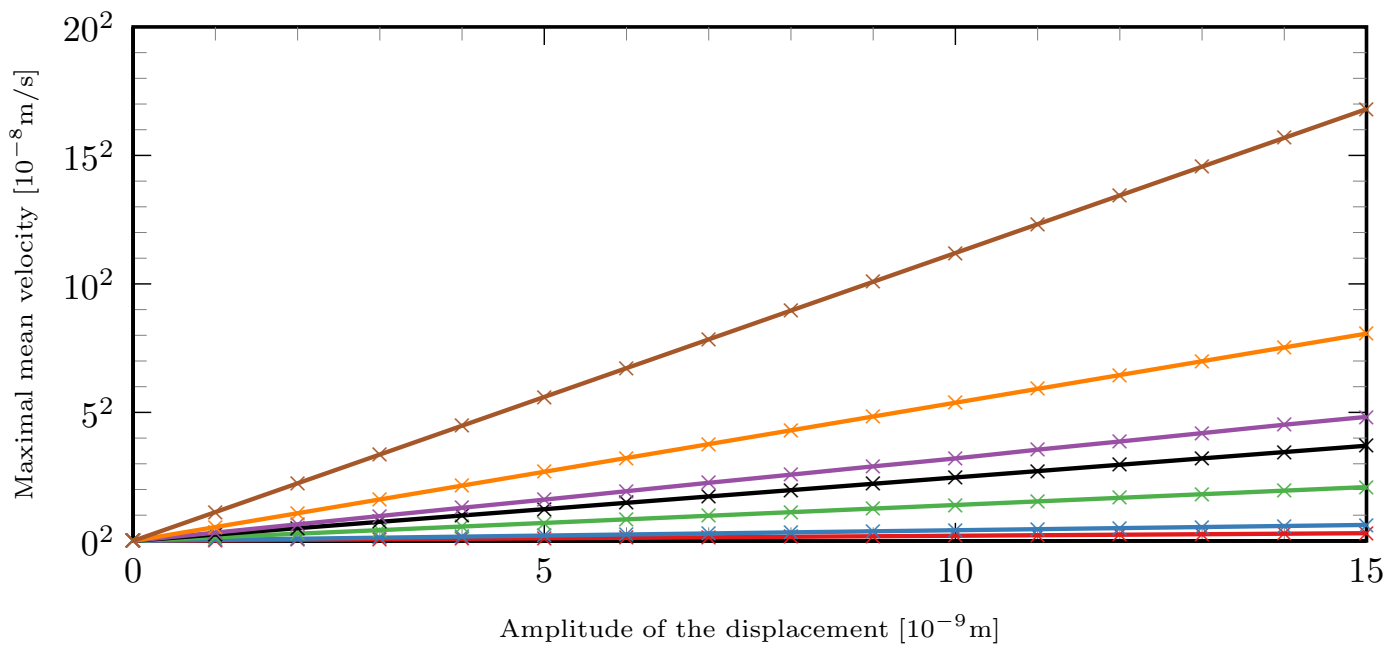

(a)

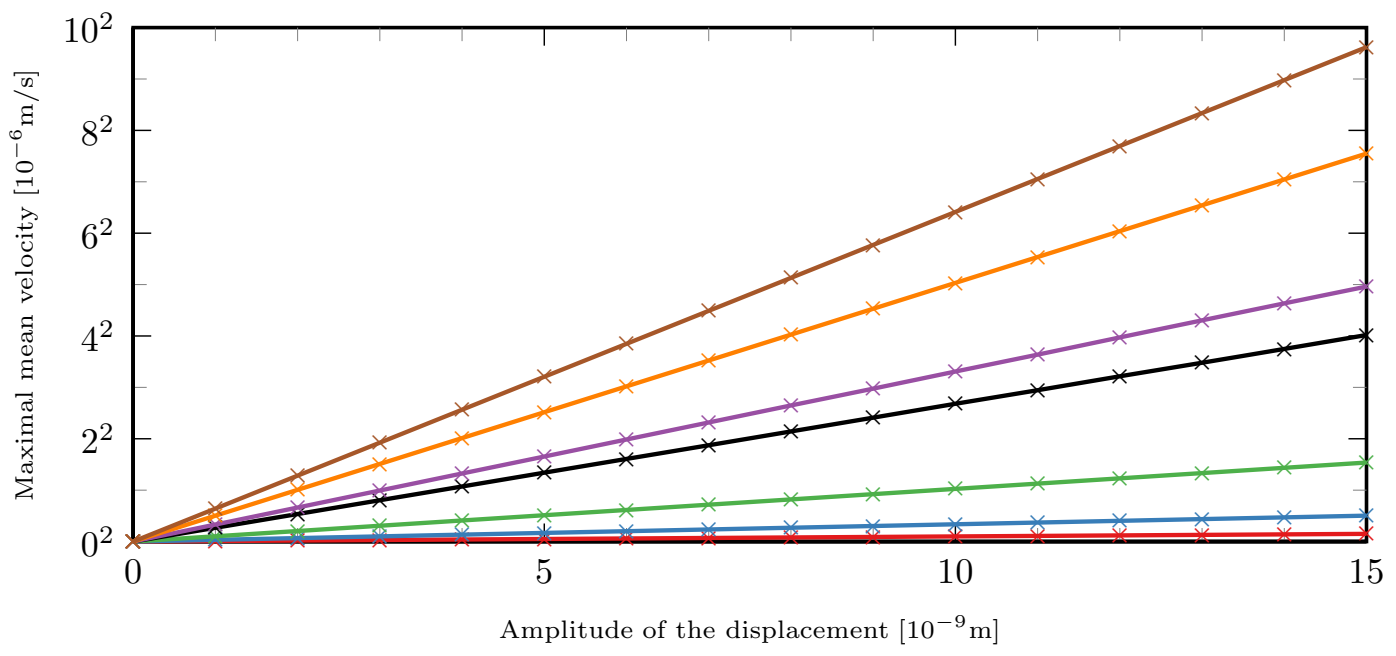

(b)

Figure 4.16: Maximal mean velocity (Lagrangian specification) at different stimulation frequencies and stimulation amplitudes in the passive cochlea model (a) as well as in the active model $(\mathrm{b})$ : $128 \mathrm{~Hz}$ (red line), $256 \mathrm{~Hz}$ (blue line), $512 \mathrm{~Hz}$ (green line), $1024 \mathrm{~Hz}$ (black line), $2048 \mathrm{~Hz}$ (purple line), $4096 \mathrm{~Hz}$ (orange line), $8192 \mathrm{~Hz}$ (brown line). 


\section{Chapter 5}

\section{Conclusion}

This work was concerned with the numerical simulation of acoustically driven flows within the inner ear. It is the first time that the occurrence and magnitude of acoustic streaming was successfully analyzed on the basis of a computational model. The relevance of acoustic streaming with respect to its physiological impact on the mechanisms of hearing is still an open question. Therefore, the results of this work provide instruments in order to support the discussion about the influence of nonlinear flow effects within the cochlea.

Up to now, acoustically driven flows were either examined by performing experiments on mechanical models (cf. [48]) or on the basis of analytical considerations (cf. [20, 25]). As mentioned by Tonndorf [48], mechanical models have the advantage of allowing direct observation of nonlinear mean flows. But due to a different scaling of the mechanical model in relation to the human cochlea, the experiments can only provide information about the principle structure of a potential mean flow. Furthermore, the mechanical model has some significant limitations concerning its usability, for example with respect to the stimulation amplitude and the frequency range.

In contrast, Lighthill [25] analytically derived an estimate of the of magnitude of acoustic streaming outside the Stokes boundary layer near to the characteristic place. But his analytical model is based on substantial simplifications and it does not describe the concrete flow field of the secondary mean motion.

The computational model presented in this work overcomes most of these difficulties. On the one hand the model has been performed on the basis of realistic dimensions with regard to the physical properties and, furthermore, there has been no constraints concerning the usability as in the case of mechanical model. On the other hand, the simplifications made by the modeling process are not as substantial as in an analytical model. In this context it should be emphasized that the computational model provides information about both the structure of the secondary flow field as well as the magnitude of the mean motion.

In comparison to other cochlea models, a very comprehensive and complex model has been developed in order to be able to simulate the acoustically driven flows within the inner ear. The complexity of the presented model is the result of the explicit consideration 
of the dynamical behavior of three major components: the fluid, the basilar membrane and the outer hair cell motility.

Particular attention has been paid to an appropriate representation of the fluid in order to ensure that the system yields accurate results with respect to the linear and nonlinear flow motions. The dynamics of the fluid was simulated by using concepts from the field of continuum mechanics.

The consideration of the interactions of the fluid with the biological structure that separates the scala tympani from the scala vestibular was a very important aspect in order to virtually reproduce the characteristic flow field. Therefore, the cochlear partition was modeled as an oscillatory system as suggested by Mammano and Nobili [28]. But in contrast to the model from Mammano and Nobili, the external hydrodynamic forces have been directly calculated on the basis of the fluid dynamics and not with the help of a phenomenological approach. While the dynamical reaction of the basilar membrane was evaluated in the time domain, Mammano and Nobili examined the displacements of the cochlear partition in the frequency domain.

The displacement pattern of the basilar membrane and also the flow field of the fluid significantly differ between the active case, where the outer hair cell motility is taken into account, and the passive case, where this amplification mechanism is neglected. Therefore the effect of the outer hair cell motility on the resultant secondary flow field has also been analyzed. The additional force exerted by the outer hair cells was calculated on the basis of an approach suggested by Mammano and Nobili (cf. [28, 33]), who modeled the amplification mechanisms as an additional oscillatory subsystem.

In summary, a set of different differential equations and boundary conditions were deduced that describe the complex dynamics of the whole cochlear system. By the use of a well-known approach from the perturbation theory, it was possible to split the system of equations into a set of successive linear subsystems. With regard to the numerical simulation of acoustic streaming, this perturbation approach was so far only used in the context of pure fluidic systems. Therefore, it is the first time that this approach was extended to an highly fluid-structure coupled problem like the cochlea.

The resultant first order subsystem describes the linear acoustic reaction of the system. The spatial discretization of this acoustic system, performed by means of the finite element method, resulted in a system of ordinary differential equations which, in turn, was discretized by using an implicit integration method. Due to the strong coupling between the fluid, the basilar membrane and the outer hair cell motility, the different processes were synchronously solved by a monolithic approach. The simulation of this linear acoustic reaction was a very computationally intensive part of the whole process, but the accurate calculation of the first order solution was a crucial requirement for the successful determination of the second order mean flows.

The results of this first order subsystem were validated against experimental studies, analytical considerations and other models. It has been shown that an harmonic stimulation at the oval window induces the expected typical traveling wave motion of the 
cochlear partition. Furthermore, the relationship between the stimulation frequency and the associated characteristic places complies with empirical experiments (cf. [18]). It has been proven that the main cause for acoustically driven flows can be found in a boundary layer driven mechanism. Therefore, particular attention has been paid to the fluid motions within the thin Stokes boundary layer of the cochlear partition, which comply, in the main, with analytical approximations developed by Lighthill [25] on the basis of theoretical considerations from Lord Rayleigh [35, §352].

It turned out that if the outer hair cell motility is taken into account by the simulation process, the traveling wave pattern will, inter alia, be locally enhanced near to the characteristic place. This significant amplification is, in addition, accompanied with an increased phase lag and an apical peak shift as also observed by Mammano and Nobili [28] in their computational model. It should be noted that the linearized model of the outer hair cell motility can not be used to reproduce the nonlinear behavior of the amplification mechanism. Nevertheless, it has been shown that this linear model was an appropriate instrument to analyze the effect of the outer hair cell driven amplification on the resultant mean flows.

The time-averaged second order subsystem yielded a first order approximation of the acoustically driven flows within the inner ear. The application of the finite element method resulted in a stationary system of equations where the right hand vector includes the results of the first order system in terms of a virtual force- and mass-source distribution.

It turned out that the resultant acoustic streaming flow field is in accordance with the experimental studies from Tonndorf [48. Furthermore, the results of the numerical simulations indicate that the maximal velocity of the acoustically driven flows are up to one order of magnitude larger than the analytical estimates from Lighthill [25].

This work opens up new opportunities in the context of investigations with respect to the bio-mechanics of hearing. Furthermore, the model shows potential for further improvements with regard to convergence and accuracy. Also an extension for three-dimensional examinations would be desirable. 


\section{Appendix A}

\section{Results of the Passive Cochlea Model}

\section{A.1 Initial Transient Effect}

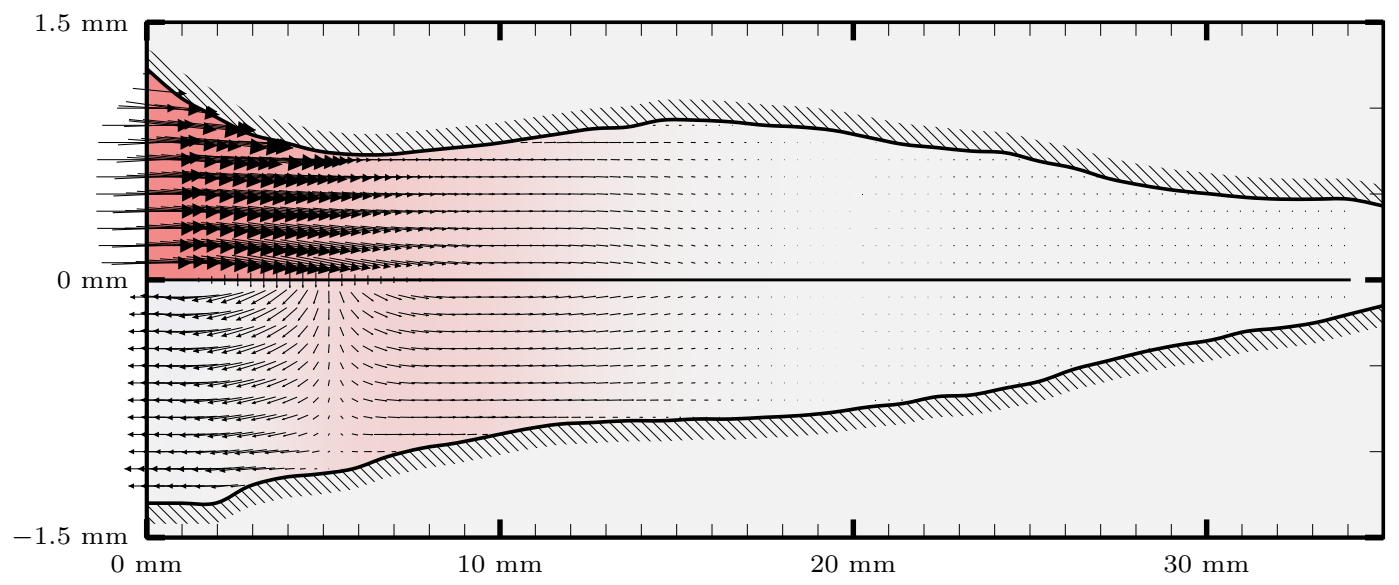

Figure A.1: Current state of the cochlea system after $\frac{1}{4}$ T seconds at 1024Hz stimulation frequency. The vector field illustrates the velocity $\boldsymbol{v}^{(1)}$ whereas the intensity of the red (or blue) color represents the amount of the positive (or negative) pressure $p^{(1)}$. 


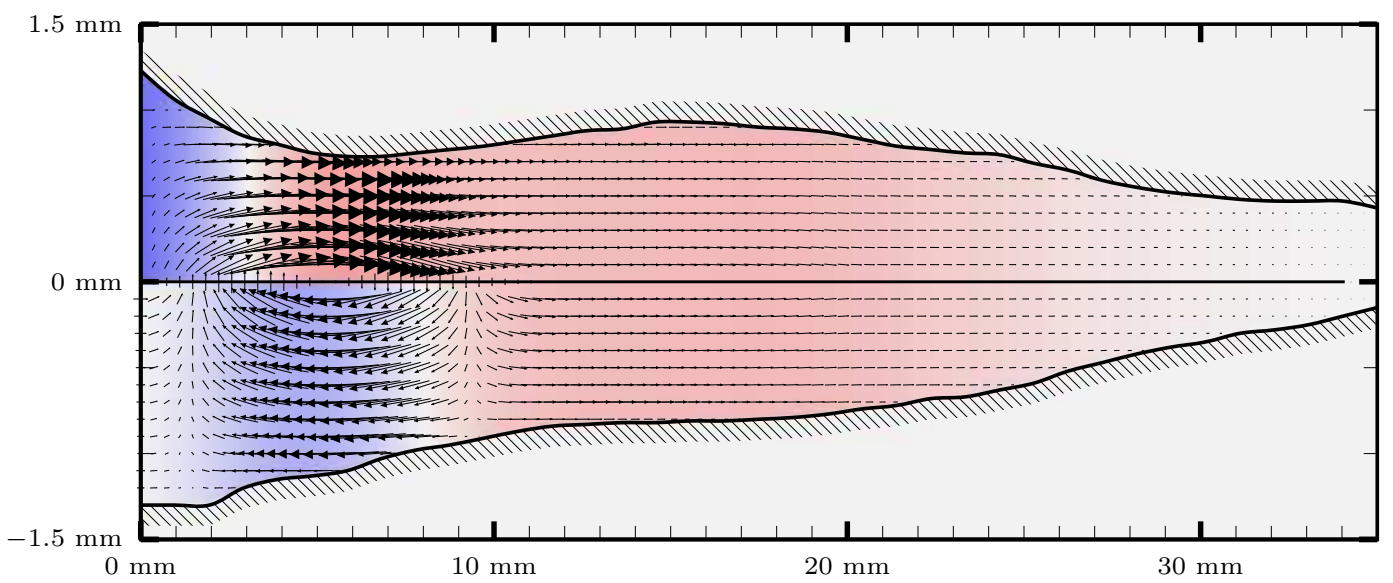

Figure A.2: Current state of the cochlea system after $\frac{1}{2} T$ seconds at 1024Hz stimulation frequency. The vector field illustrates the velocity $\boldsymbol{v}^{(1)}$ whereas the intensity of the red (or blue) color represents the amount of the positive (or negative) pressure $p^{(1)}$.

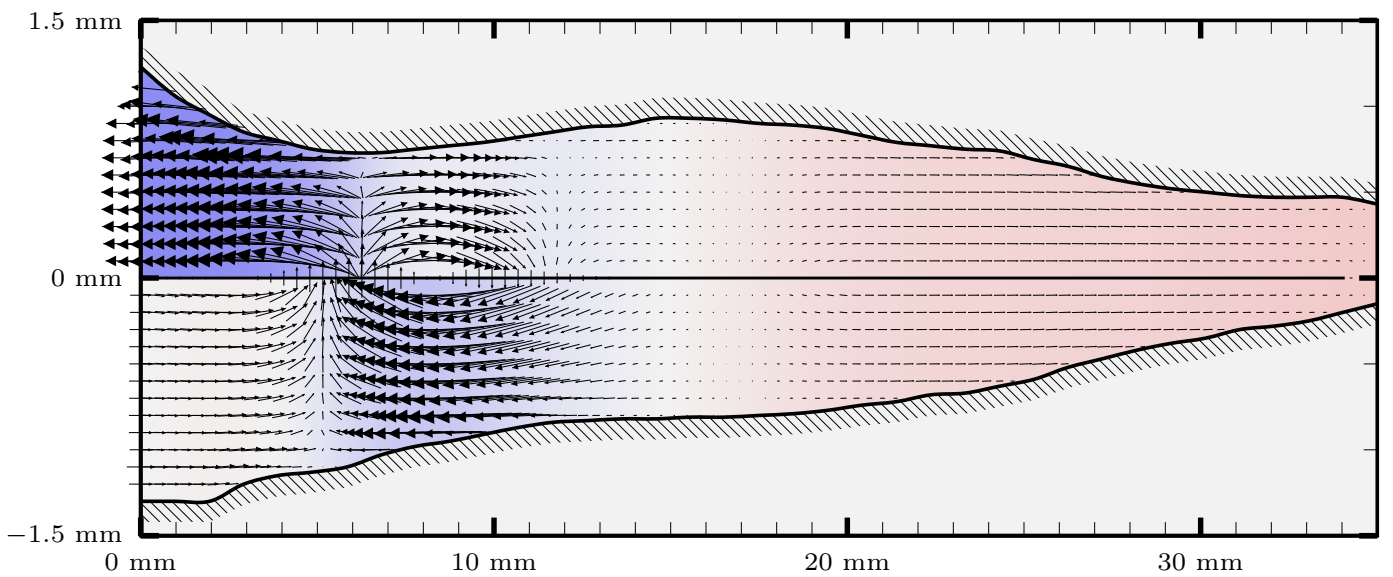

Figure A.3: Current state of the cochlea system after $\frac{3}{4}$ T seconds at $1024 \mathrm{~Hz}$ stimulation frequency. The vector field illustrates the velocity $\boldsymbol{v}^{(1)}$ whereas the intensity of the red (or blue) color represents the amount of the positive (or negative) pressure $p^{(1)}$. 


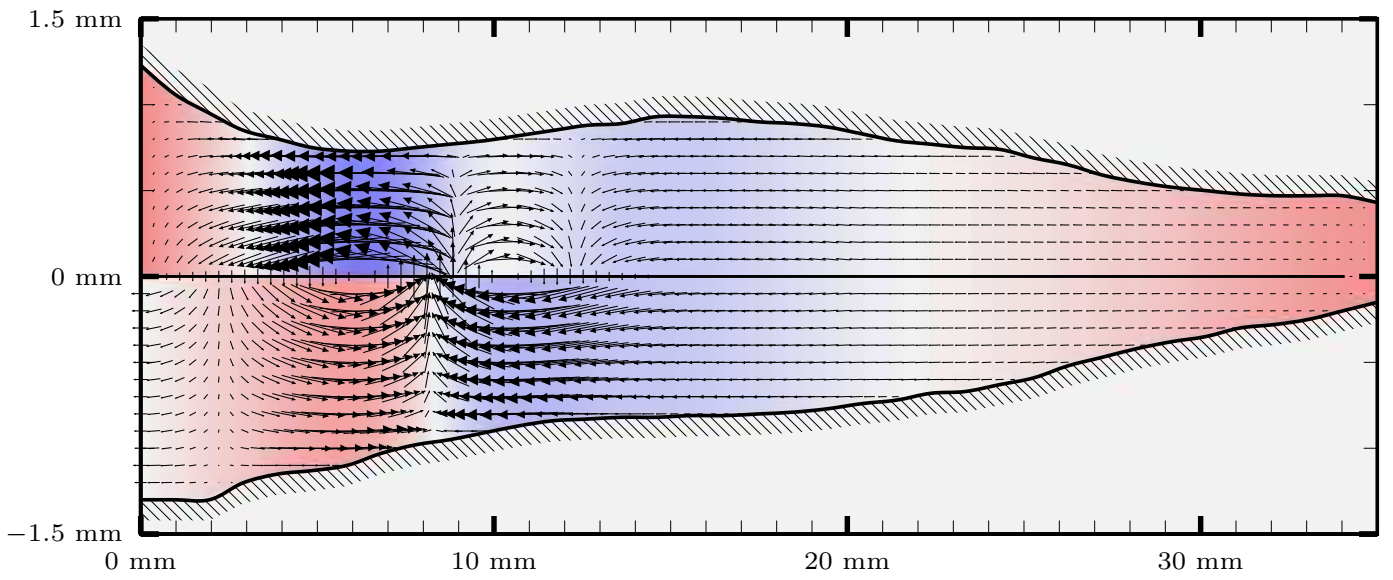

Figure A.4: Current state of the cochlea system after $T$ seconds at $1024 \mathrm{~Hz}$ stimulation frequency. The vector field illustrates the velocity $\boldsymbol{v}^{(1)}$ whereas the intensity of the red (or blue) color represents the amount of the positive (or negative) pressure $p^{(1)}$.

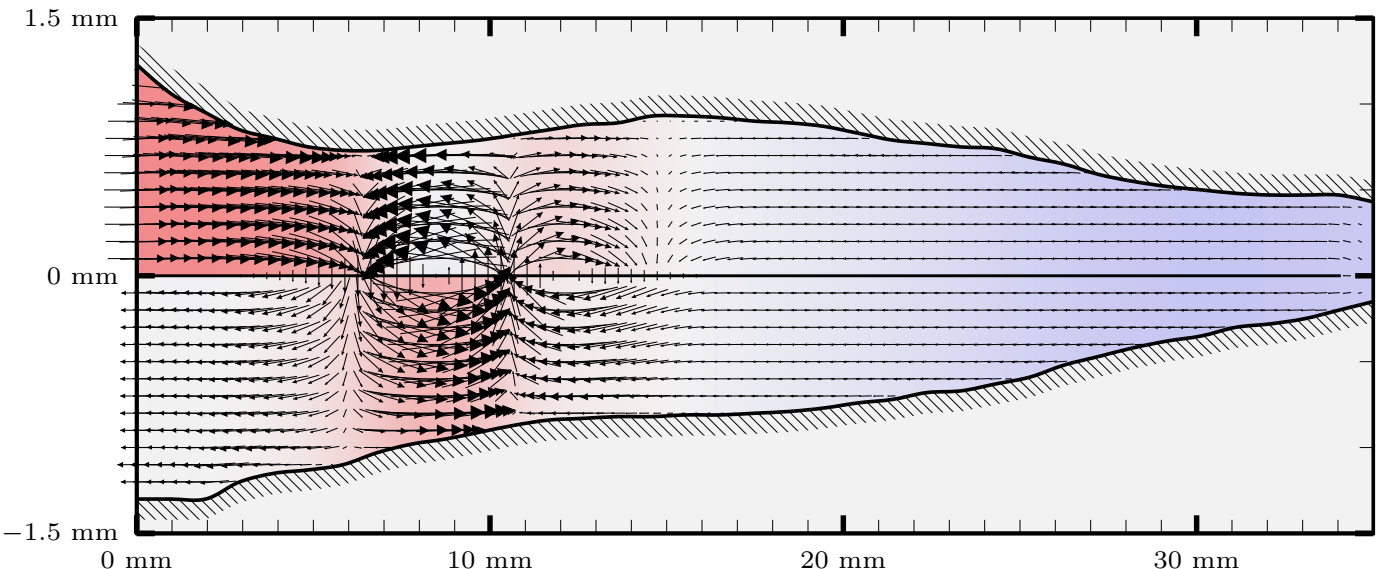

Figure A.5: Current state of the cochlea system after $\frac{5}{4}$ T seconds at 1024Hz stimulation frequency. The vector field illustrates the velocity $\boldsymbol{v}^{(1)}$ whereas the intensity of the red (or blue) color represents the amount of the positive (or negative) pressure $p^{(1)}$. 


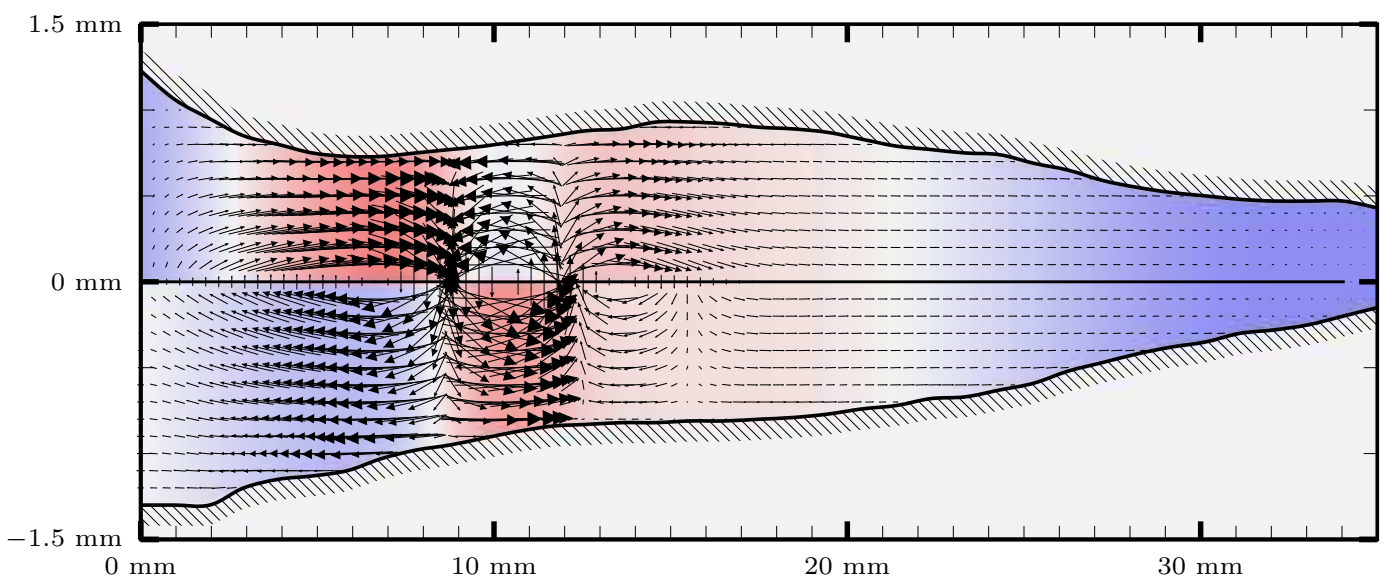

Figure A.6: Current state of the cochlea system after $\frac{3}{2} T$ seconds at $1024 \mathrm{~Hz}$ stimulation frequency. The vector field illustrates the velocity $\boldsymbol{v}^{(1)}$ whereas the intensity of the red (or blue) color represents the amount of the positive (or negative) pressure $p^{(1)}$.

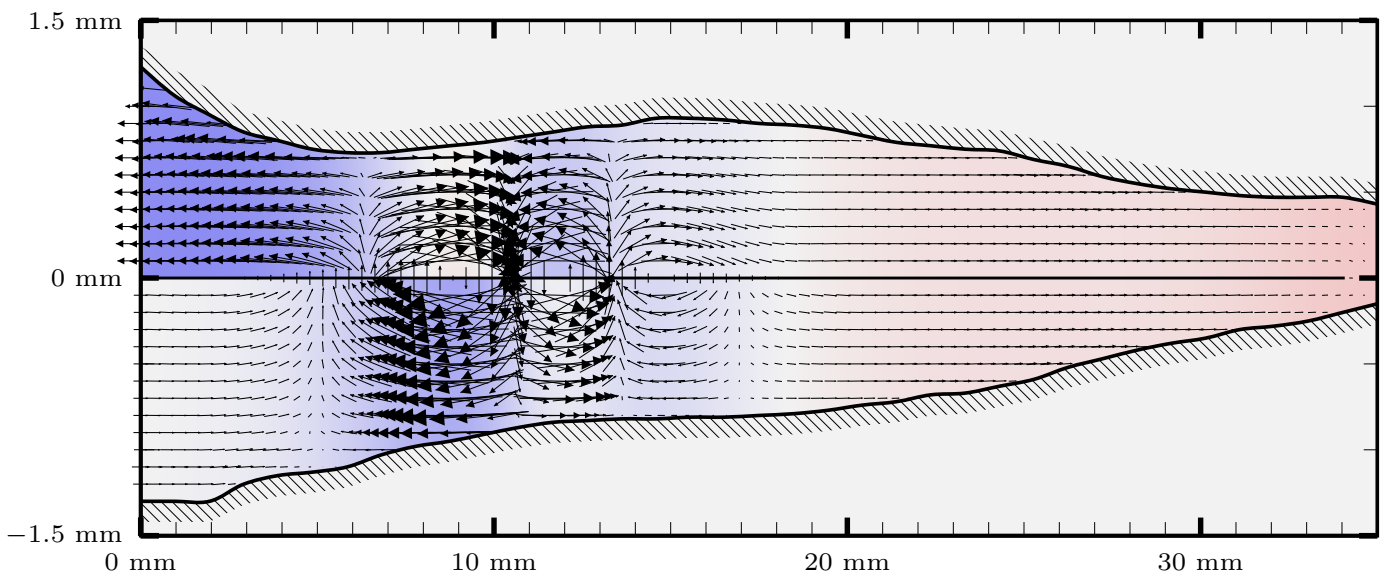

Figure A.7: Current state of the cochlea system after $\frac{7}{4} T$ seconds at 1024Hz stimulation frequency. The vector field illustrates the velocity $\boldsymbol{v}^{(1)}$ whereas the intensity of the red (or blue) color represents the amount of the positive (or negative) pressure $p^{(1)}$. 


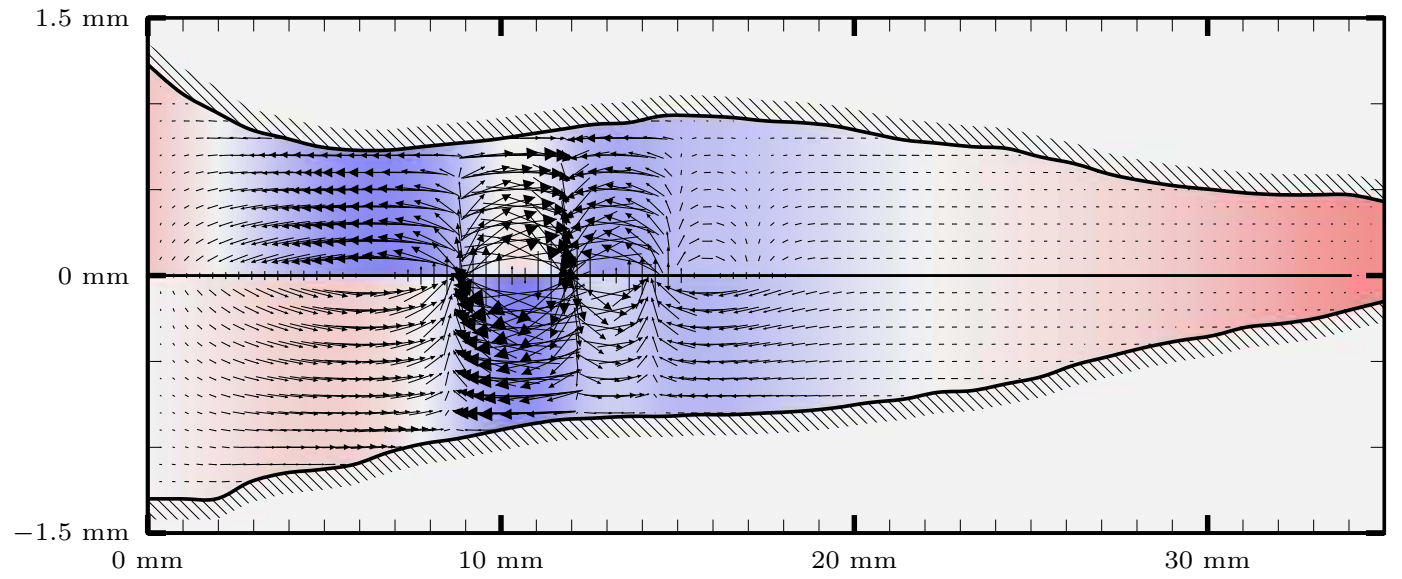

Figure A.8: Current state of the cochlea system after $2 \mathrm{~T}$ seconds at 1024Hz stimulation frequency. The vector field illustrates the velocity $\boldsymbol{v}^{(1)}$ whereas the intensity of the red (or blue) color represents the amount of the positive (or negative) pressure $p^{(1)}$. 


\section{A.2 Rotary Vibrations at Equilibrium State}

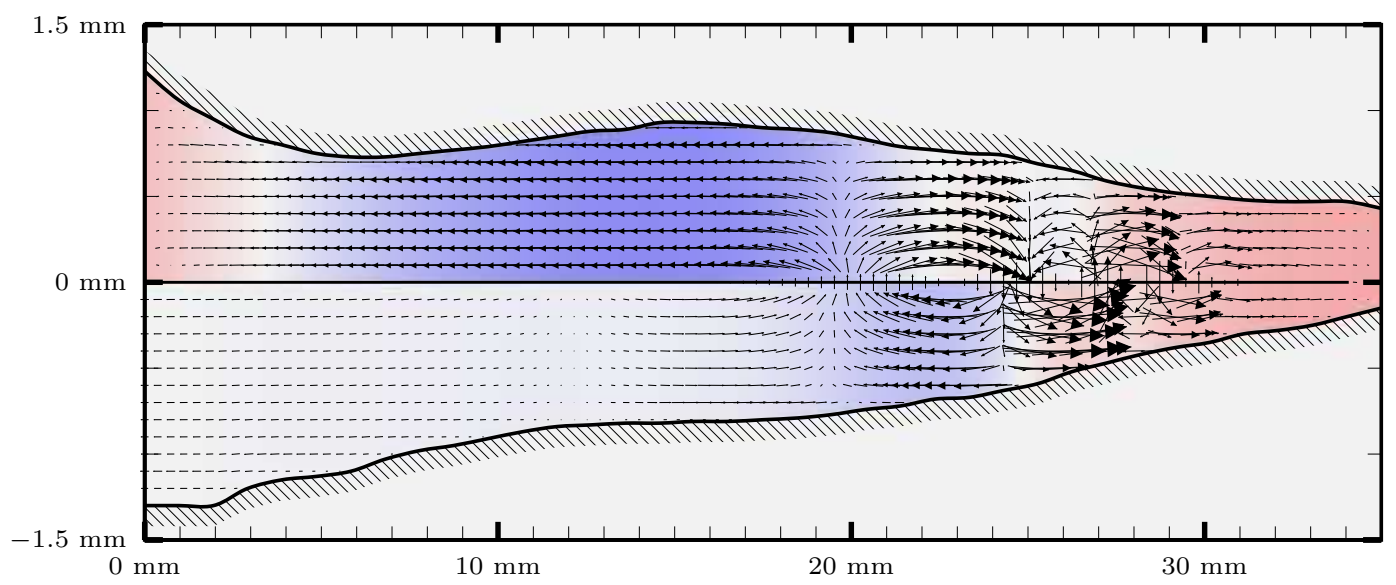

(a)

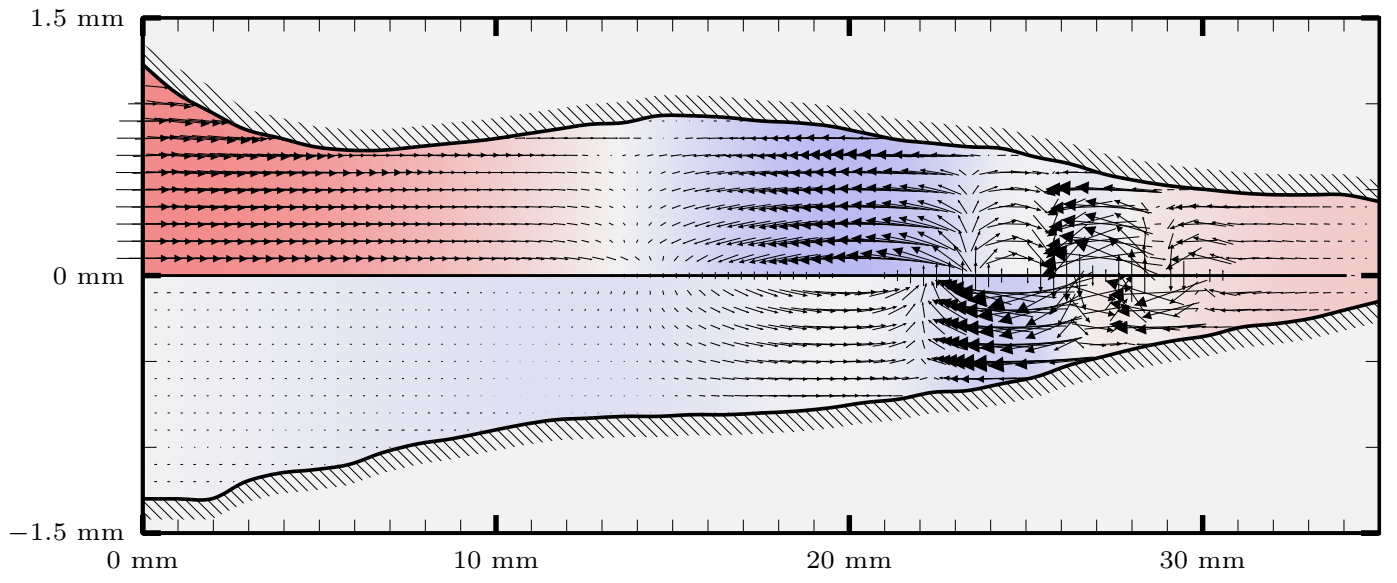

(b)

Figure A.9: Equilibrium state of the first order system at the stimulation frequency of $128 \mathrm{~Hz}$ in the passive cochlea model. Figure (a) illustrates the real part of the velocity field $\boldsymbol{v}^{\text {(cos) }}$ of the rotary vibration in combination with the real pressure field $p^{(\mathrm{cos})}$. Figure (b) visualizes their imaginary counterparts $\boldsymbol{v}^{(\mathrm{sin})}$ and $p^{(\mathrm{sin})}$. The intensity of the red (blue) color represents the amount of the positve (negative) pressure. 


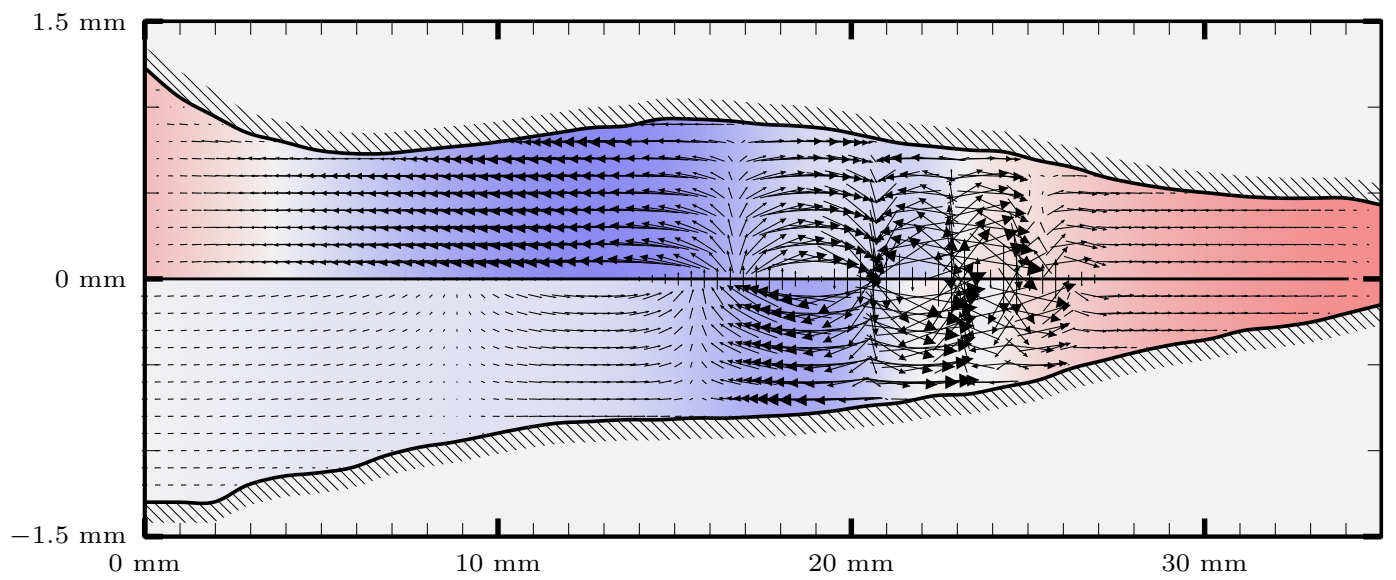

(a)

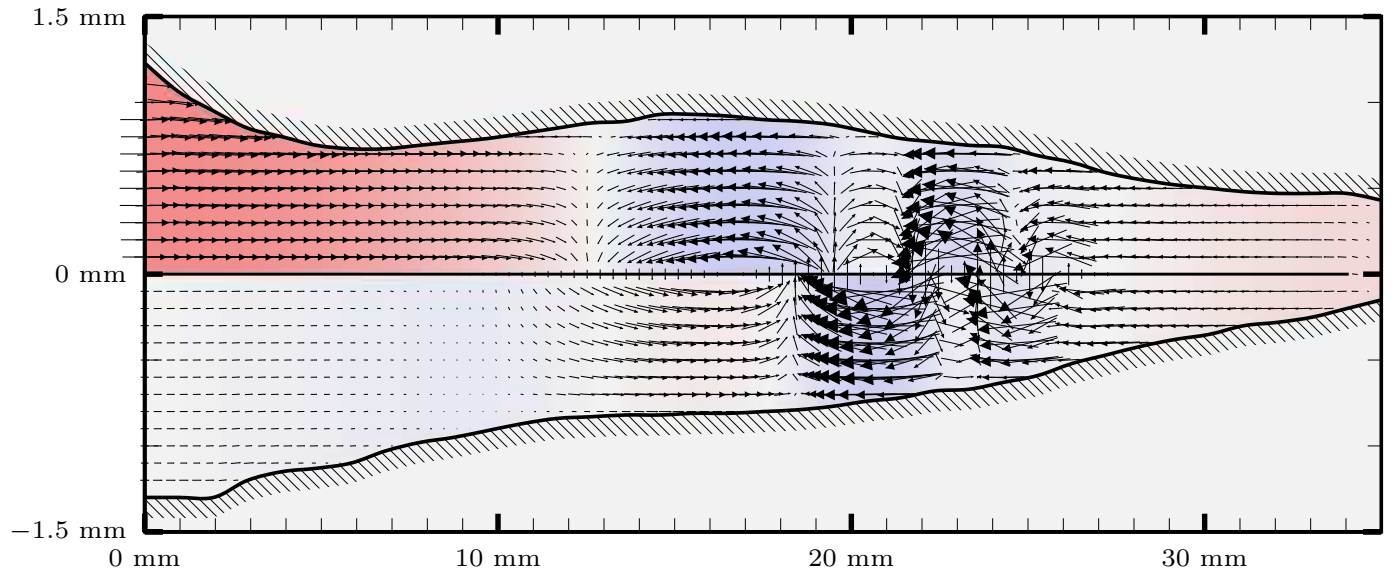

(b)

Figure A.10: Equilibrium state of the first order system at the stimulation frequency of $256 \mathrm{~Hz}$ in the passive cochlea model. Figure (a) illustrates the real part of the velocity field $\boldsymbol{v}^{\text {(cos) }}$ of the rotary vibration in combination with the real pressure field $p^{(\mathrm{cos})}$. Figure (b) visualizes their imaginary counterparts $\boldsymbol{v}^{(\mathrm{sin})}$ and $p^{(\sin )}$. The intensity of the red (blue) color represents the amount of the positve (negative) pressure. 


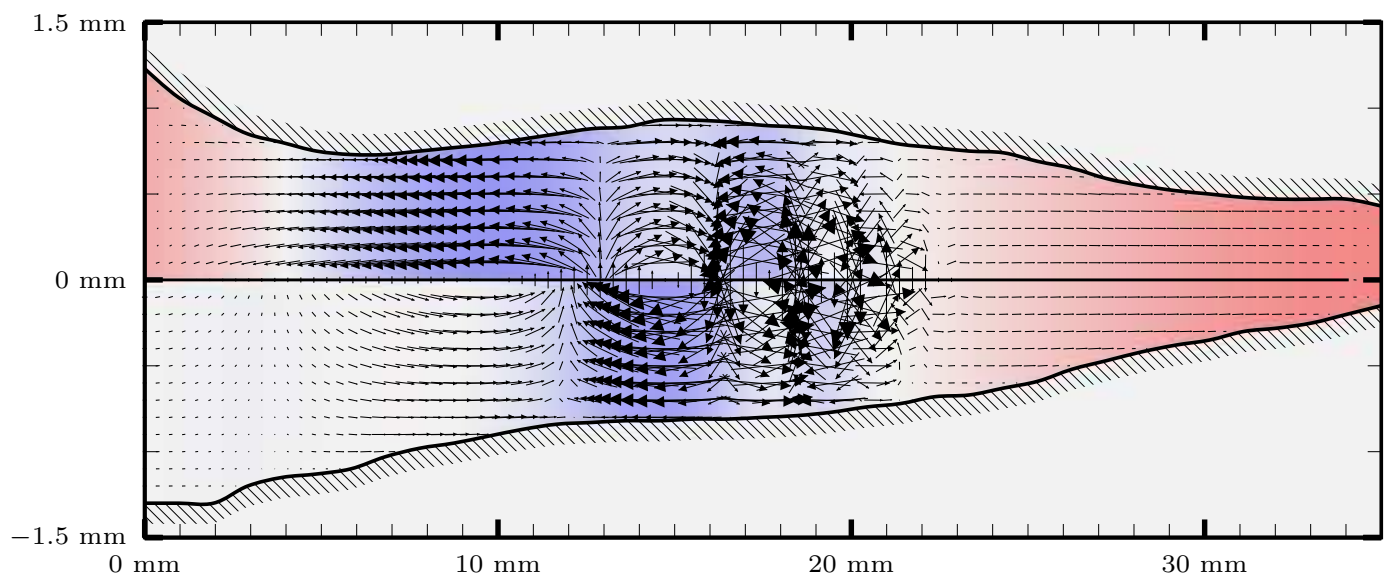

(a)

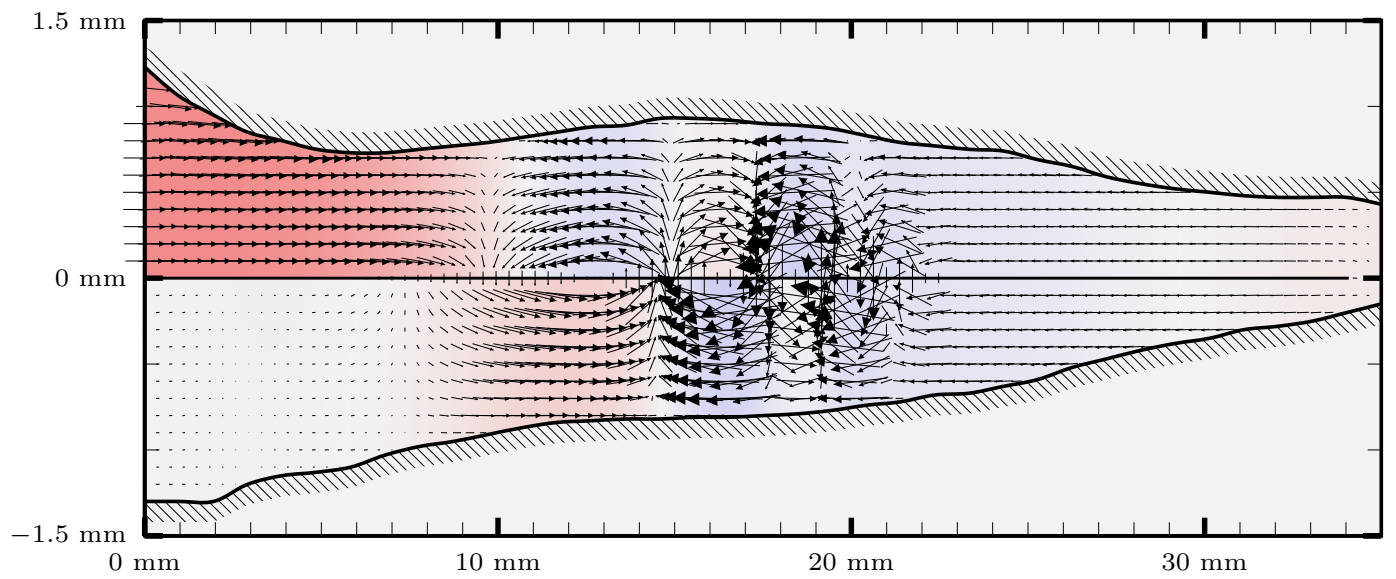

(b)

Figure A.11: Equilibrium state of the first order system at the stimulation frequency of 512Hz in the passive cochlea model. Figure (a) illustrates the real part of the velocity field $\boldsymbol{v}^{\text {(cos) }}$ of the rotary vibration in combination with the real pressure field $p^{(\mathrm{cos})}$. Figure (b) visualizes their imaginary counterparts $\boldsymbol{v}^{(\mathrm{sin})}$ and $p^{(\sin )}$. The intensity of the red (blue) color represents the amount of the positve (negative) pressure. 


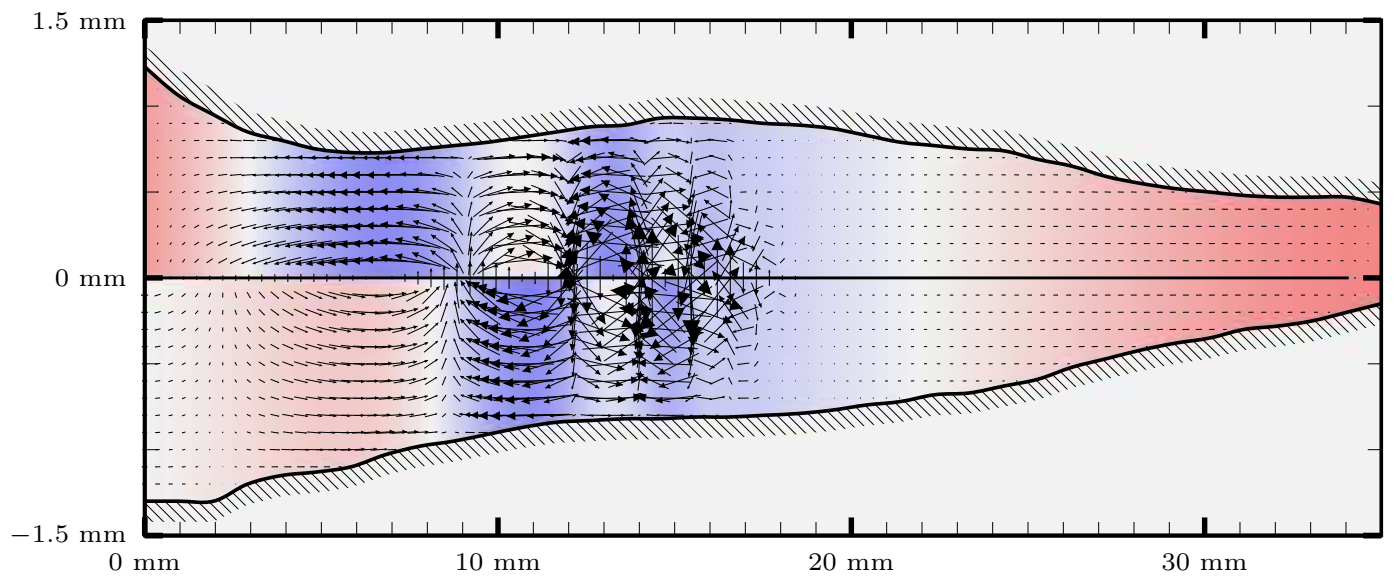

(a)

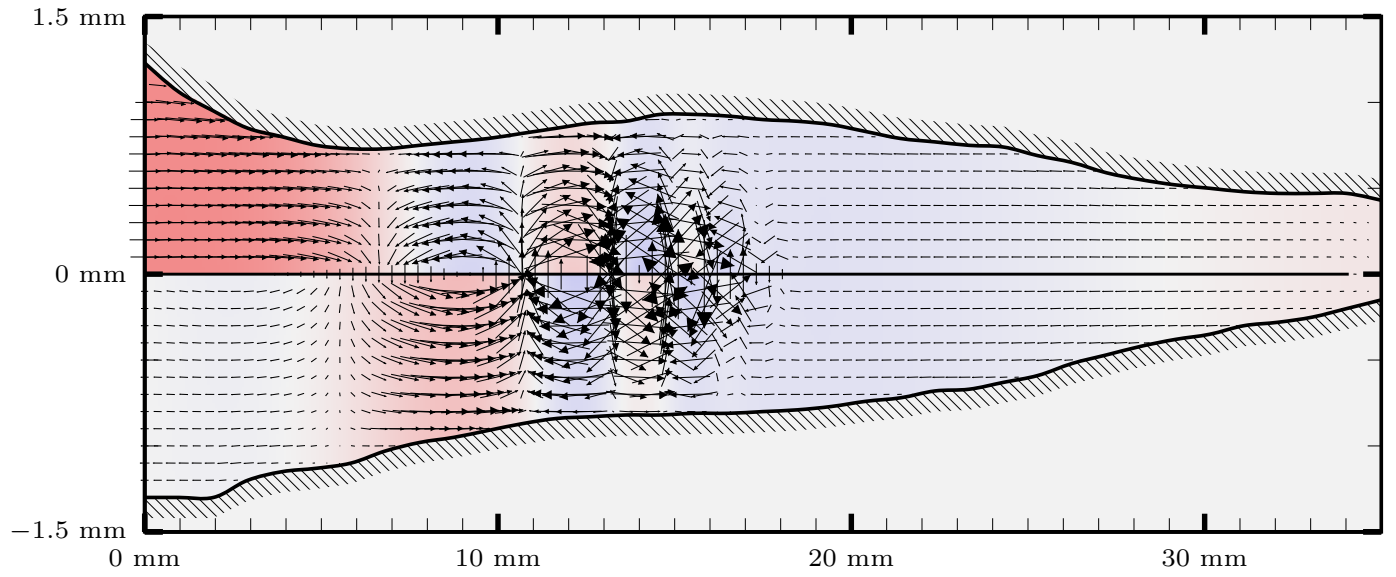

(b)

Figure A.12: Equilibrium state of the first order system at the stimulation frequency of 1024Hz in the passive cochlea model. Figure (a) illustrates the real part of the velocity field $\boldsymbol{v}^{\text {(cos) }}$ of the rotary vibration in combination with the real pressure field $p^{(\mathrm{cos})}$. Figure (b) visualizes their imaginary counterparts $\boldsymbol{v}^{(\mathrm{sin})}$ and $p^{(\sin )}$. The intensity of the red (blue) color represents the amount of the positve (negative) pressure. 


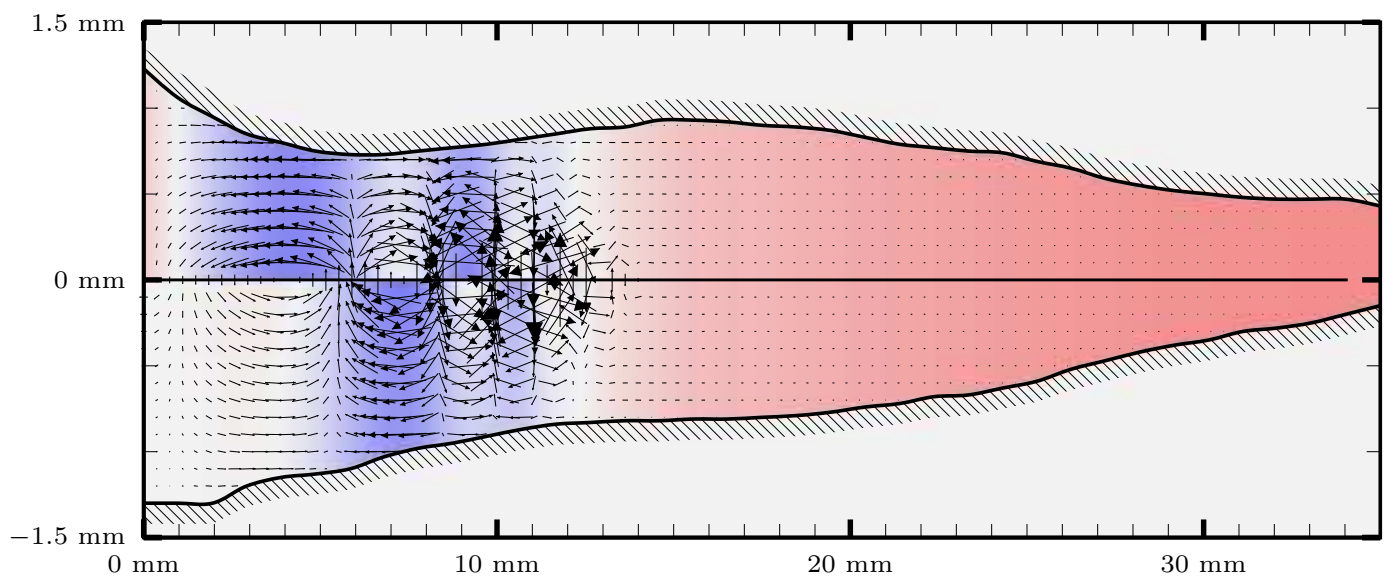

(a)

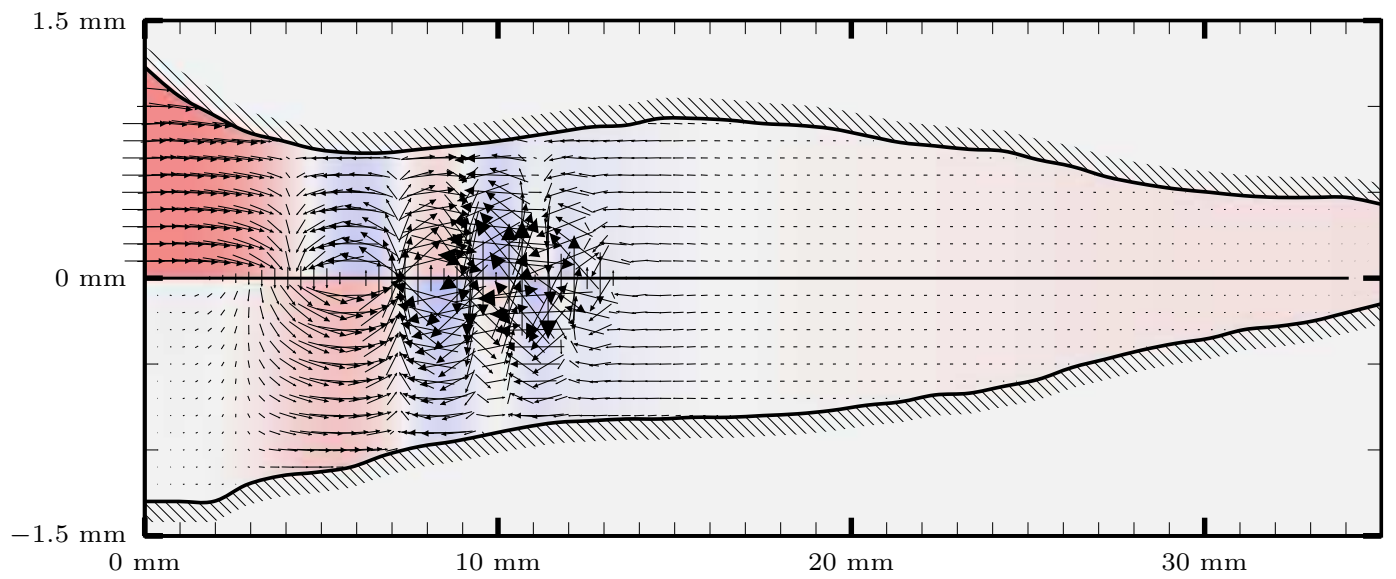

(b)

Figure A.13: Equilibrium state of the first order system at the stimulation frequency of $2048 \mathrm{~Hz}$ in the passive cochlea model. Figure (a) illustrates the real part of the velocity field $\boldsymbol{v}^{(\mathrm{cos})}$ of the rotary vibration in combination with the real pressure field $p^{(\mathrm{cos})}$. Figure (b) visualizes their imaginary counterparts $\boldsymbol{v}^{(\mathrm{sin})}$ and $p^{(\sin )}$. The intensity of the red (blue) color represents the amount of the positve (negative) pressure. 


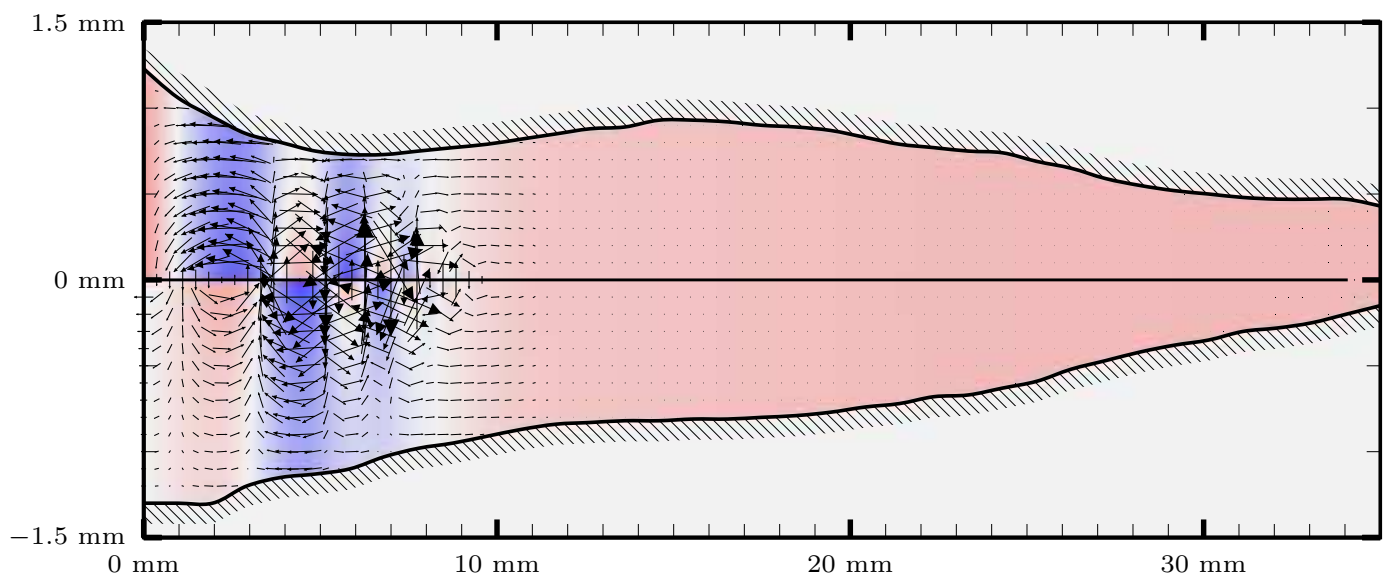

(a)

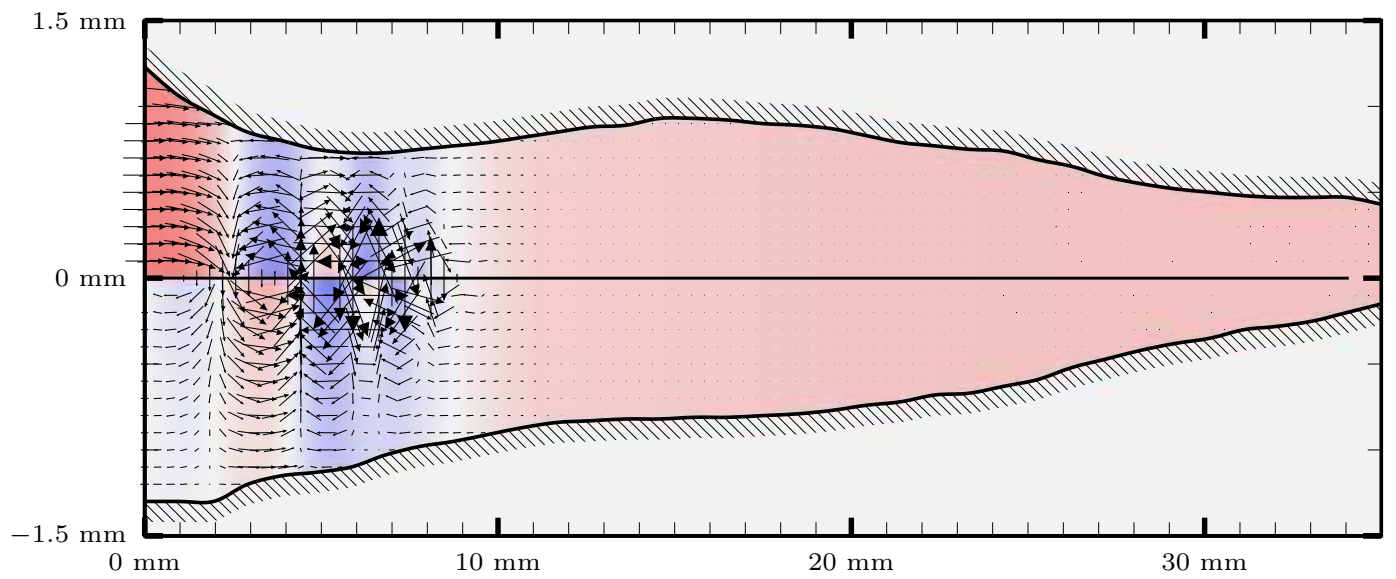

(b)

Figure A.14: Equilibrium state of the first order system at the stimulation frequency of $4096 \mathrm{~Hz}$ in the passive cochlea model. Figure (a) illustrates the real part of the velocity field $\boldsymbol{v}^{\text {(cos) }}$ of the rotary vibration in combination with the real pressure field $p^{(\mathrm{cos})}$. Figure (b) visualizes their imaginary counterparts $\boldsymbol{v}^{(\mathrm{sin})}$ and $p^{(\sin )}$. The intensity of the red (blue) color represents the amount of the positve (negative) pressure. 


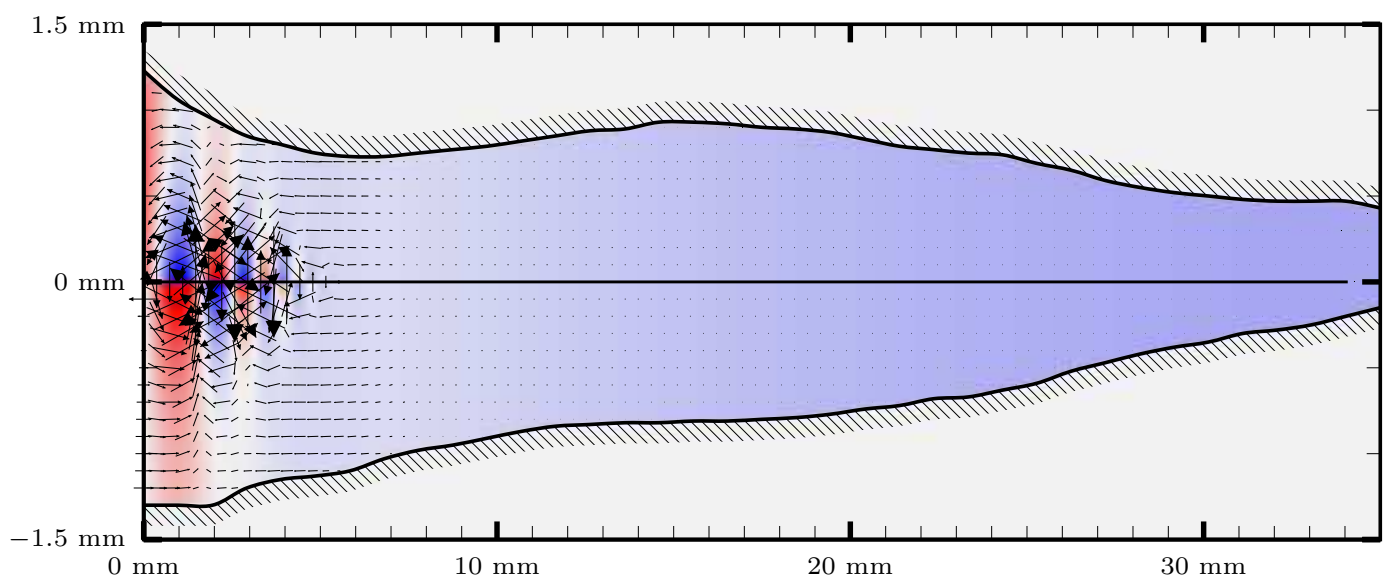

(a)

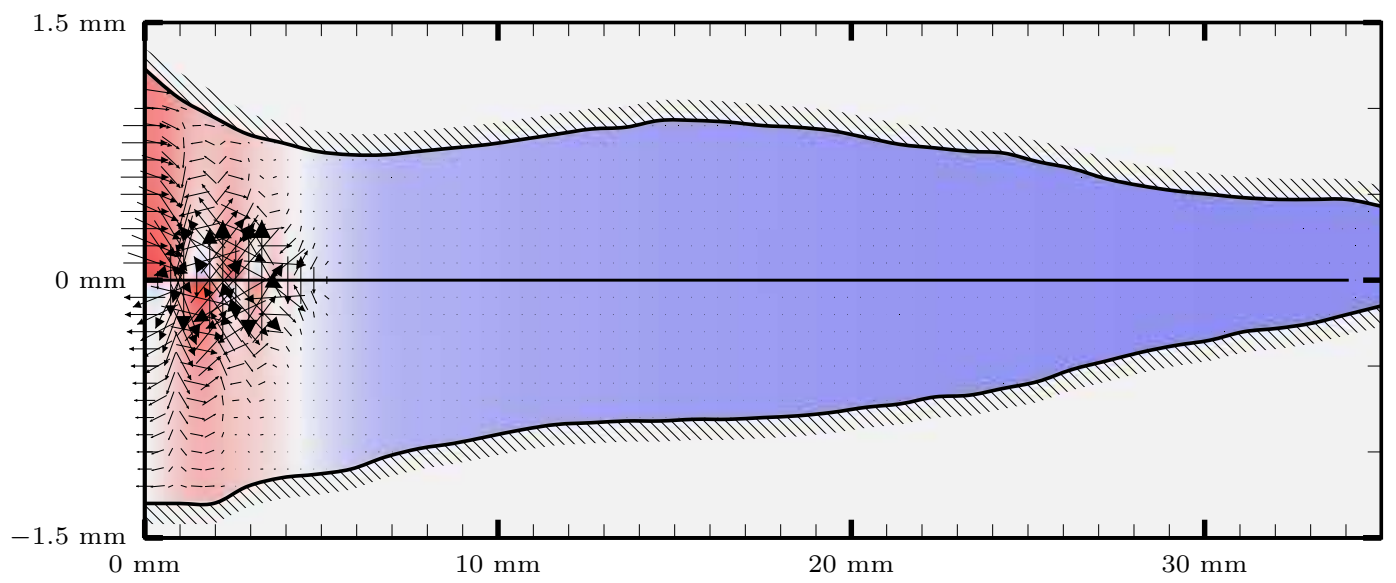

(b)

Figure A.15: Equilibrium state of the first order system at the stimulation frequency of $8192 \mathrm{~Hz}$ in the passive cochlea model. Figure (a) illustrates the real part of the velocity field $\boldsymbol{v}^{(\mathrm{cos})}$ of the rotary vibration in combination with the real pressure field $p^{(\mathrm{cos})}$. Figure (b) visualizes their imaginary counterparts $\boldsymbol{v}^{(\mathrm{sin})}$ and $p^{(\sin )}$. The intensity of the red (blue) color represents the amount of the positve (negative) pressure. 


\section{A.3 Displacement of the Basilar Membrane}

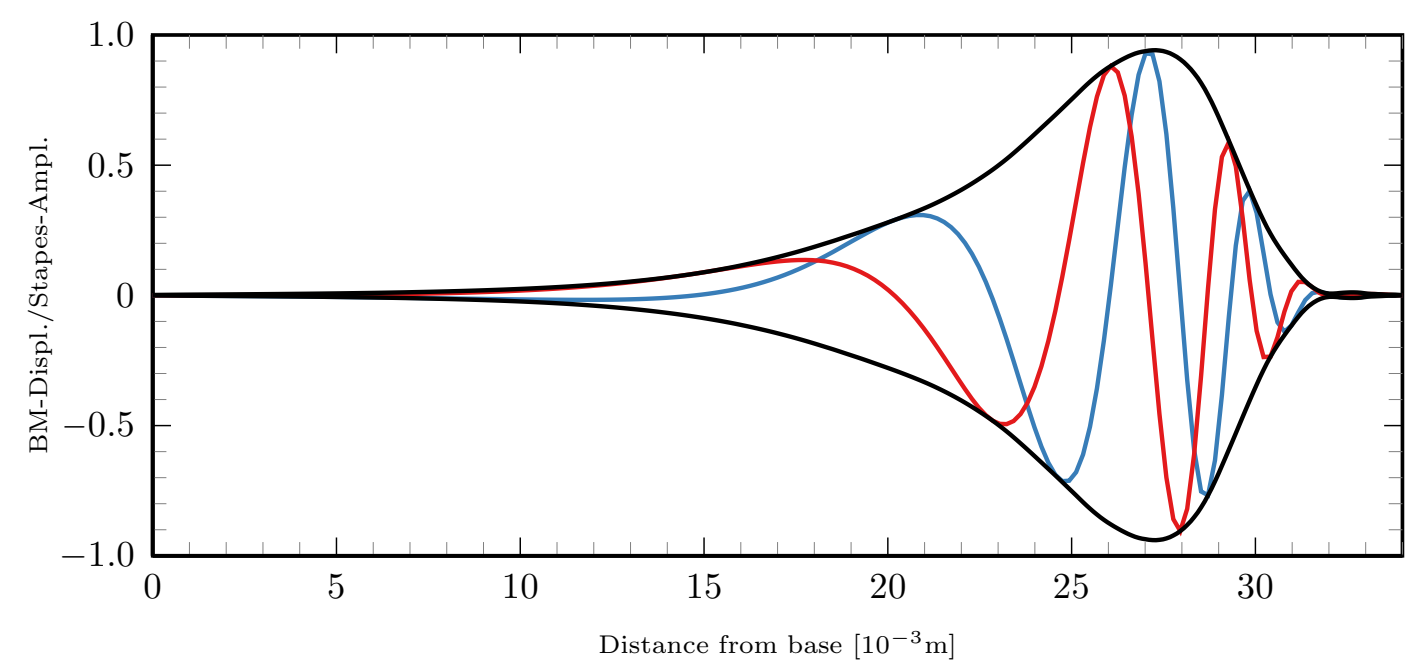

Figure A.16: Traveling wave motion of the basilar membrane at the stimulation frequency of 128Hz in the passive cochlea model. The red line represents the cosine part $\xi^{(\cos )}$ of the oscillatory motion, whereas the blue line is associated with the sine component $\xi^{(\sin )}$. The black line illustrates the envelope. 


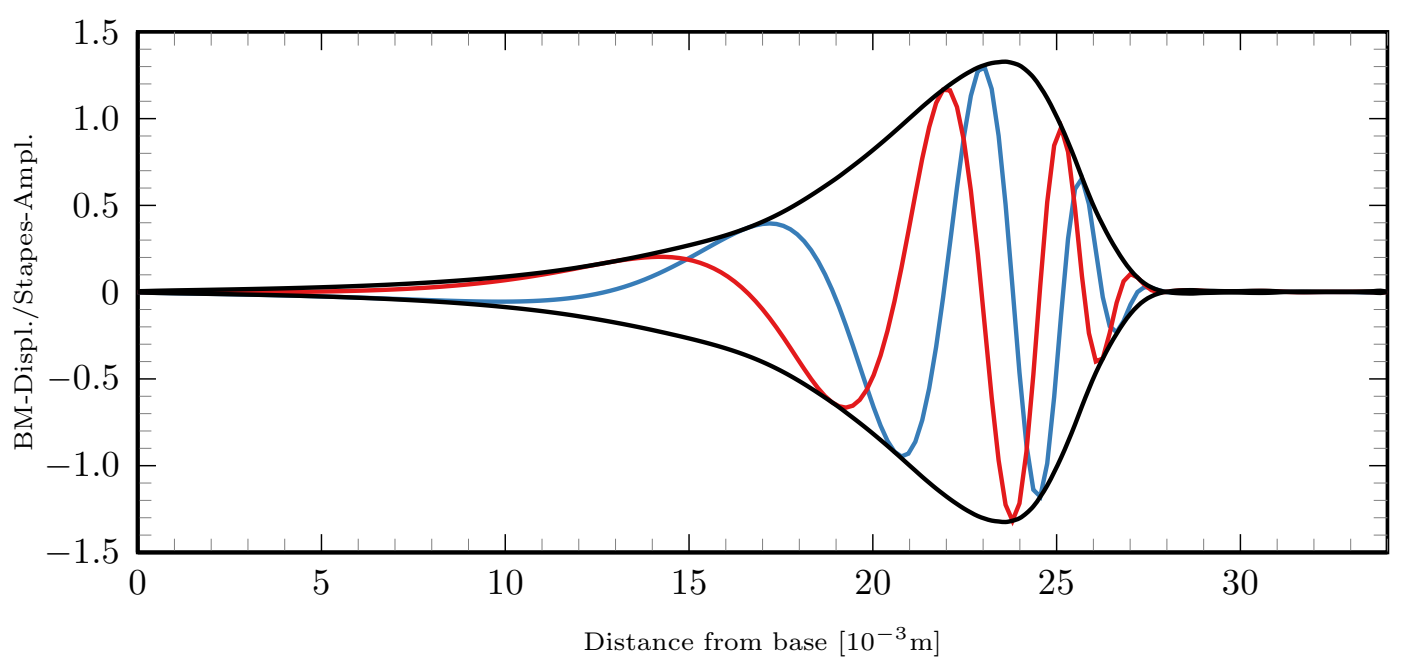

Figure A.17: Traveling wave motion of the basilar membrane at the stimulation frequency of $256 \mathrm{~Hz}$ in the passive cochlea model. The red line represents the cosine part $\xi^{(\mathrm{cos})}$ of the oscillatory motion, whereas the blue line is associated with the sine component $\xi^{(\sin )}$. The black line illustrates the envelope.

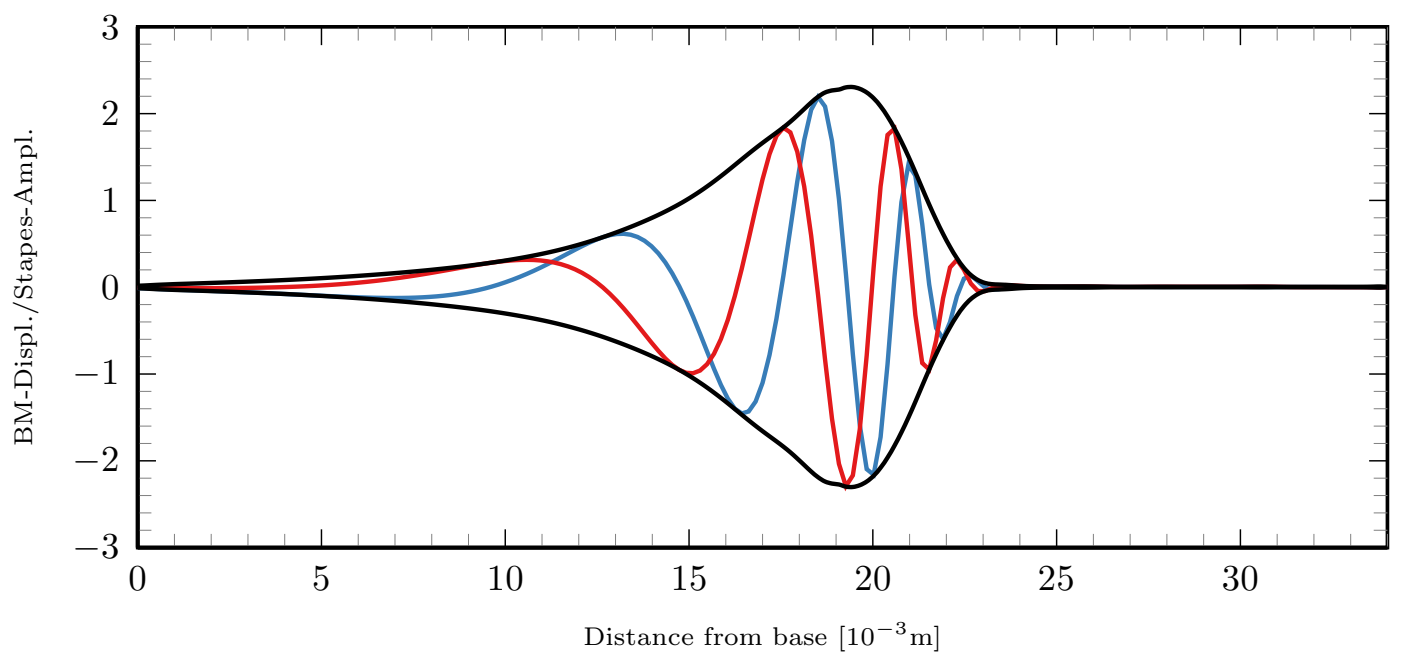

Figure A.18: Traveling wave motion of the basilar membrane at the stimulation frequency of 512Hz in the passive cochlea model. The red line represents the cosine part $\xi^{(\cos )}$ of the oscillatory motion, whereas the blue line is associated with the sine component $\xi^{(\sin )}$. The black line illustrates the envelope. 


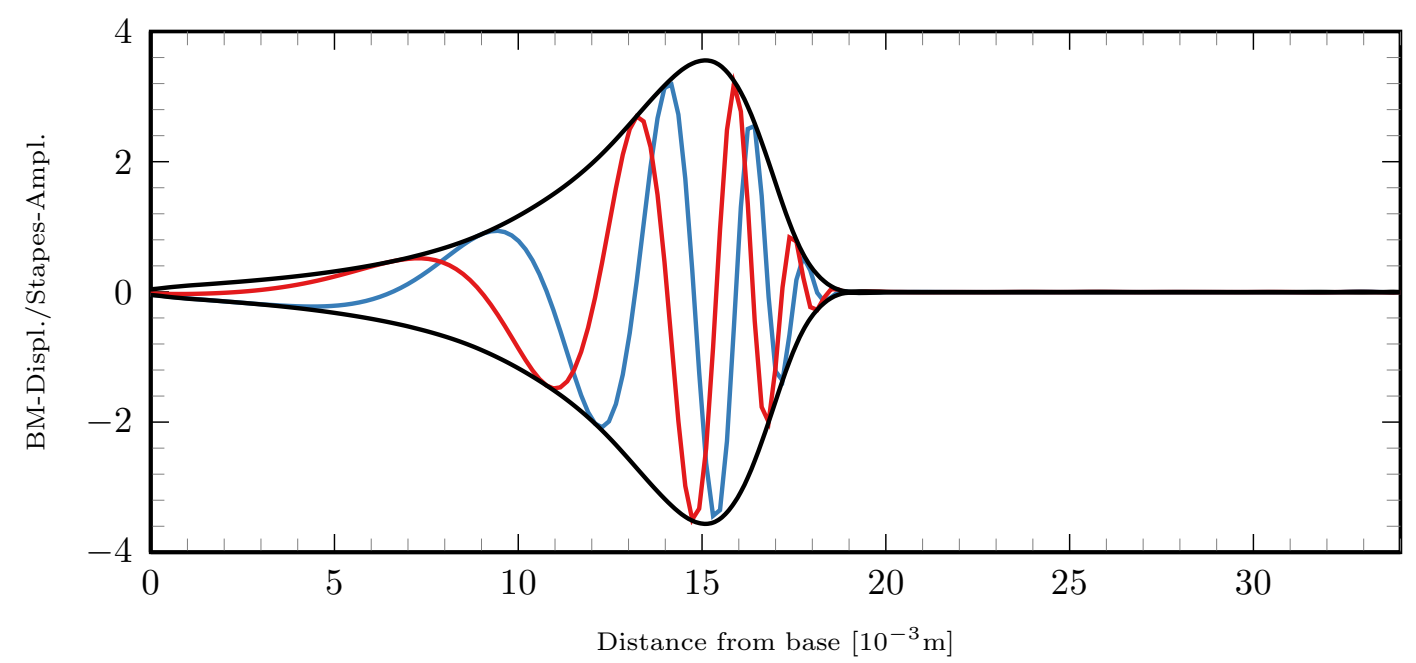

Figure A.19: Traveling wave motion of the basilar membrane at the stimulation frequency of 1024Hz in the passive cochlea model. The red line represents the cosine part $\xi^{(\cos )}$ of the oscillatory motion, whereas the blue line is associated with the sine component $\xi^{(\sin )}$. The black line illustrates the envelope.

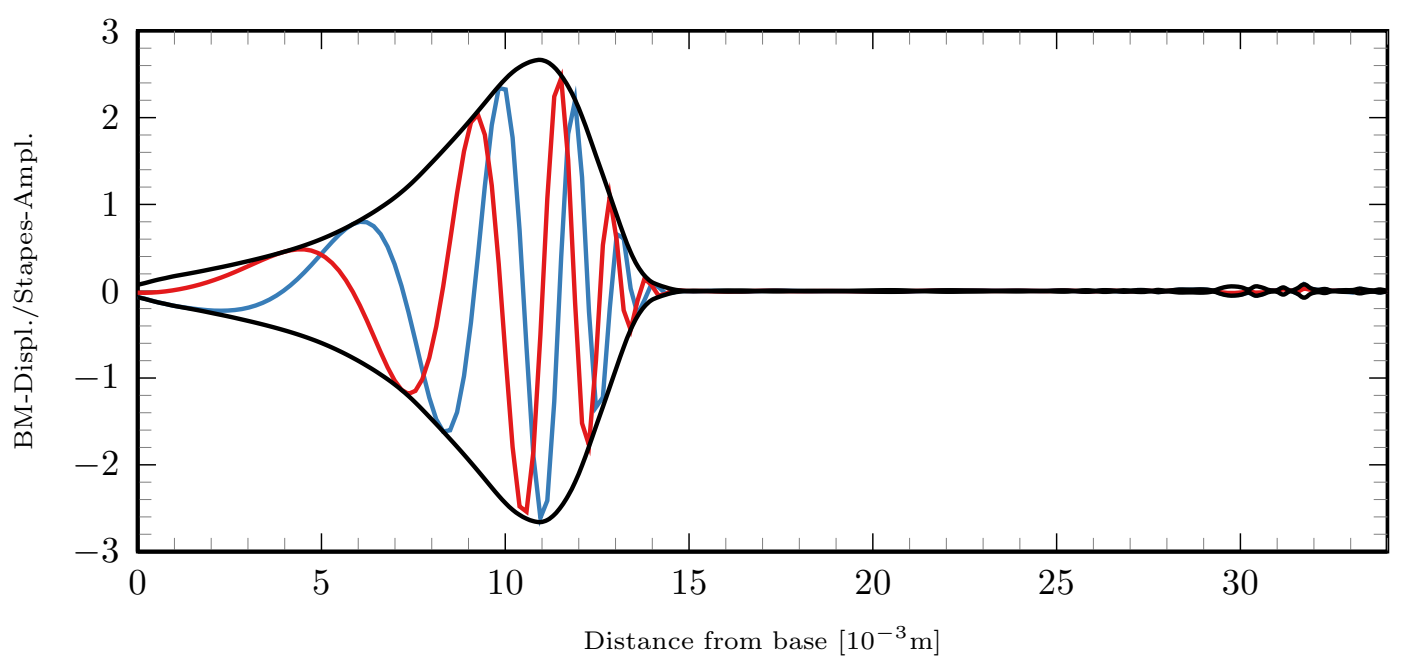

Figure A.20: Traveling wave motion of the basilar membrane at the stimulation frequency of $2048 \mathrm{~Hz}$ in the passive cochlea model. The red line represents the cosine part $\xi^{(\cos )}$ of the oscillatory motion, whereas the blue line is associated with the sine component $\xi^{(\sin )}$. The black line illustrates the envelope. 


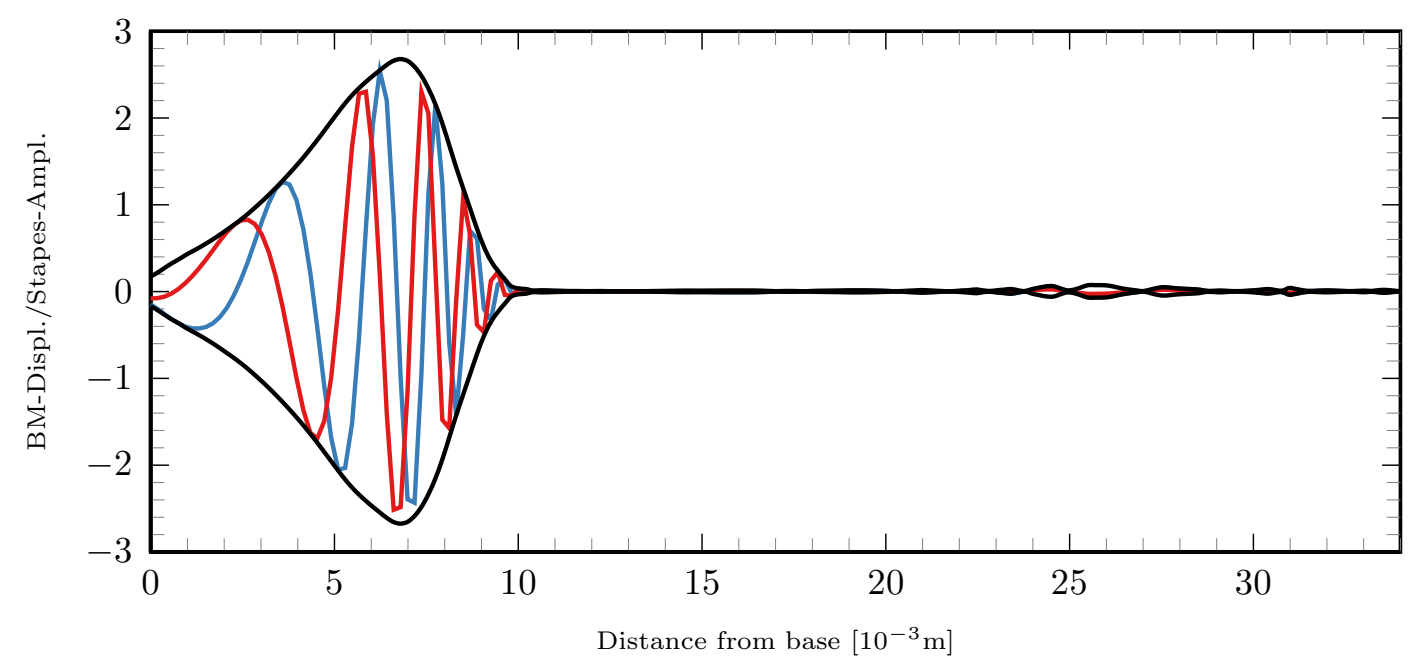

Figure A.21: Traveling wave motion of the basilar membrane at the stimulation frequency of $4096 \mathrm{~Hz}$ in the passive cochlea model. The red line represents the cosine part $\xi^{(\cos )}$ of the oscillatory motion, whereas the blue line is associated with the sine component $\xi^{(\sin )}$. The black line illustrates the envelope.

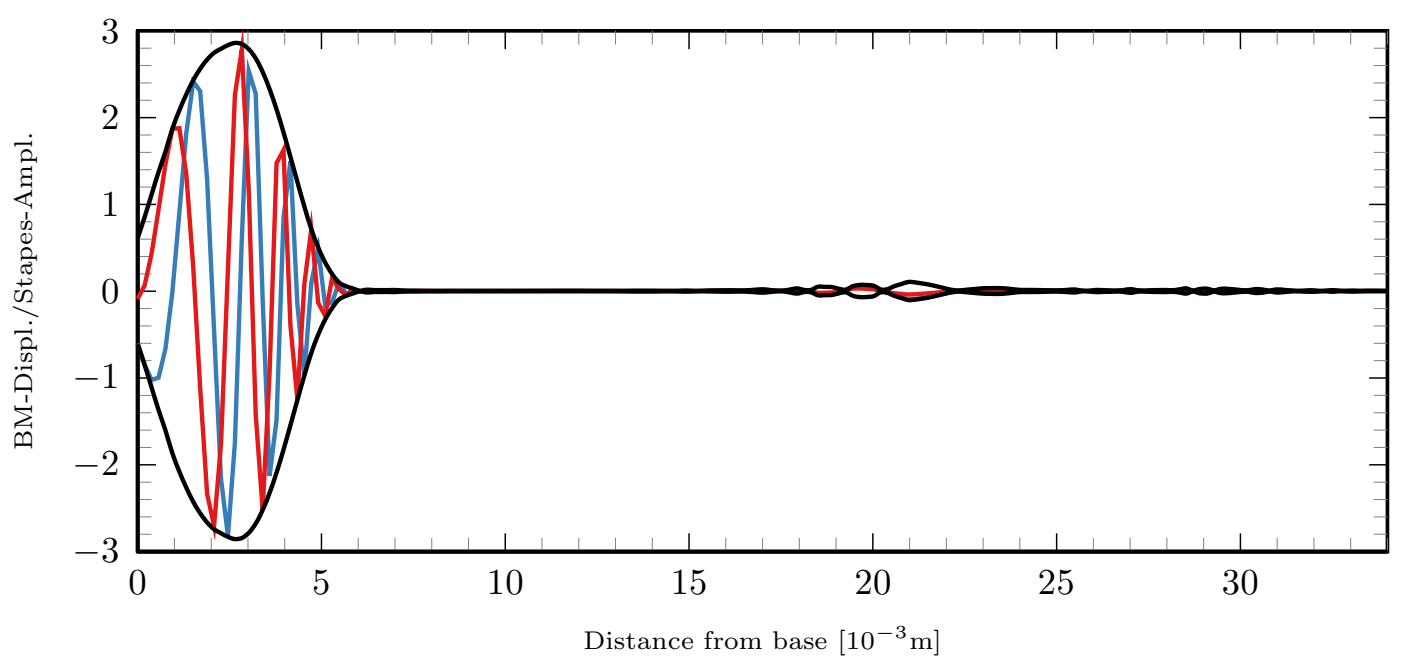

Figure A.22: Traveling wave motion of the basilar membrane at the stimulation frequency of $8192 \mathrm{~Hz}$ in the passive cochlea model. The red line represents the cosine part $\xi^{(\cos )}$ of the oscillatory motion, whereas the blue line is associated with the sine component $\xi^{(\sin )}$. The black line illustrates the envelope. 


\section{A.4 Acoustic Streaming Flow Field}

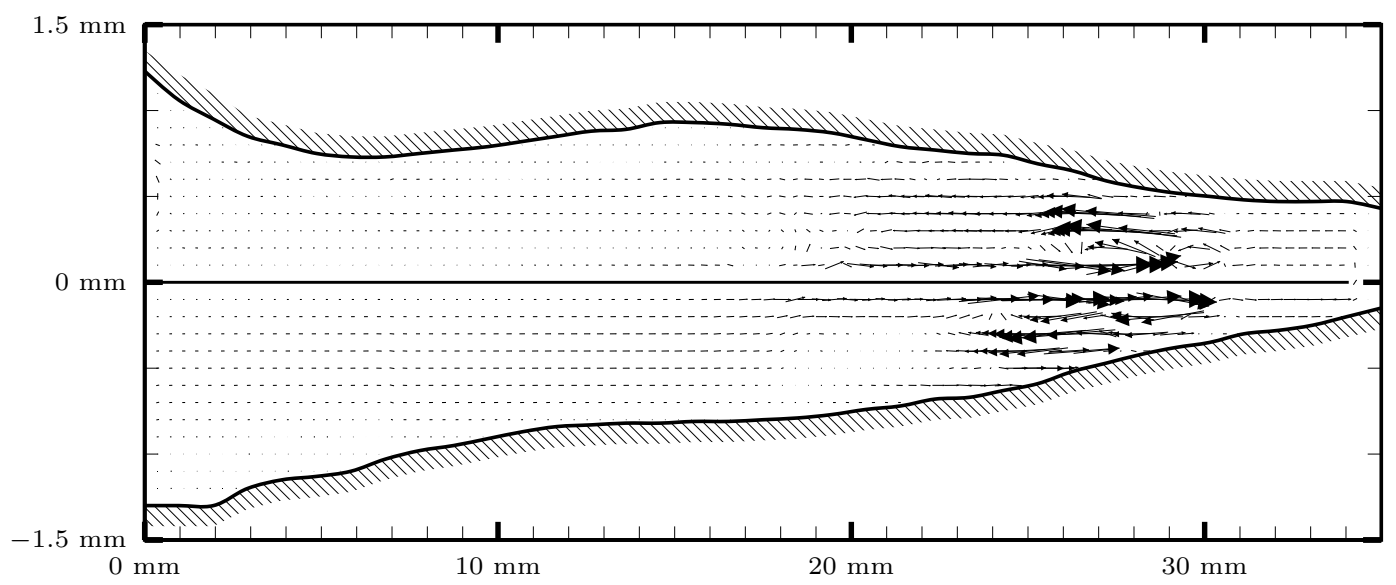

Figure A.23: Lagrangian specification of acousticically driven flows induced by a stimulation frequency of $128 \mathrm{~Hz}$ in the passive cochlea model.

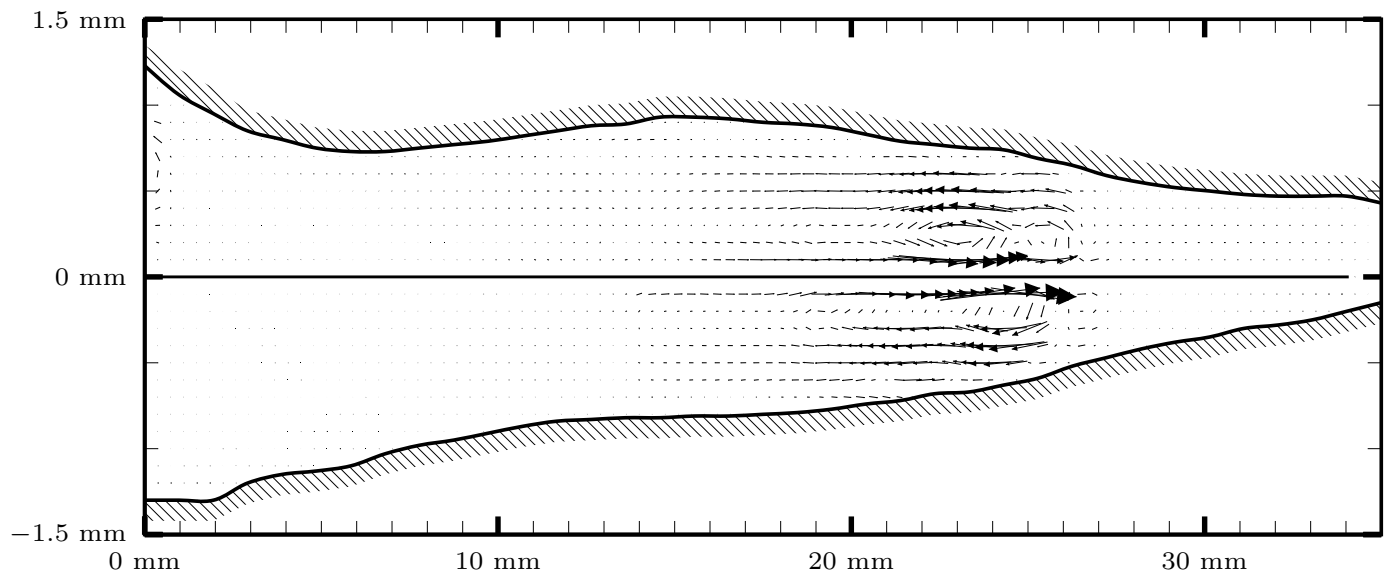

Figure A.24: Lagrangian specification of acousticically driven flows induced by a stimulation frequency of $256 \mathrm{~Hz}$ in the passive cochlea model. 


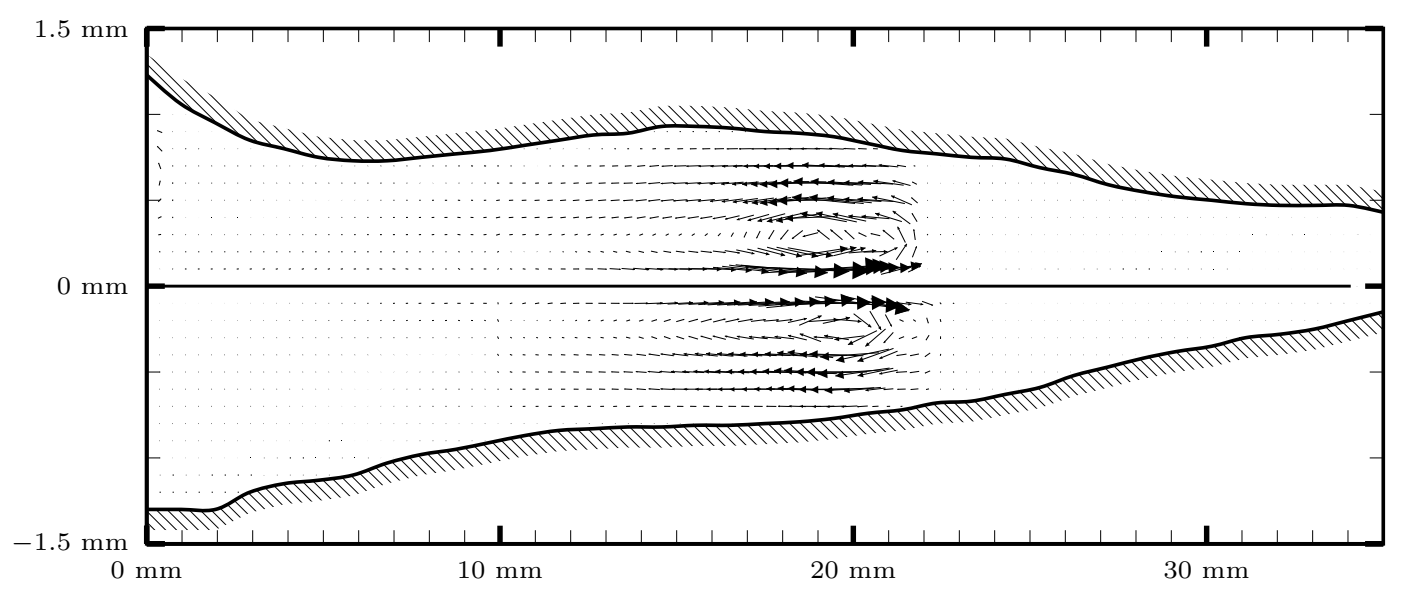

Figure A.25: Lagrangian specification of acousticically driven flows induced by a stimulation frequency of $512 \mathrm{~Hz}$ in the passive cochlea model.

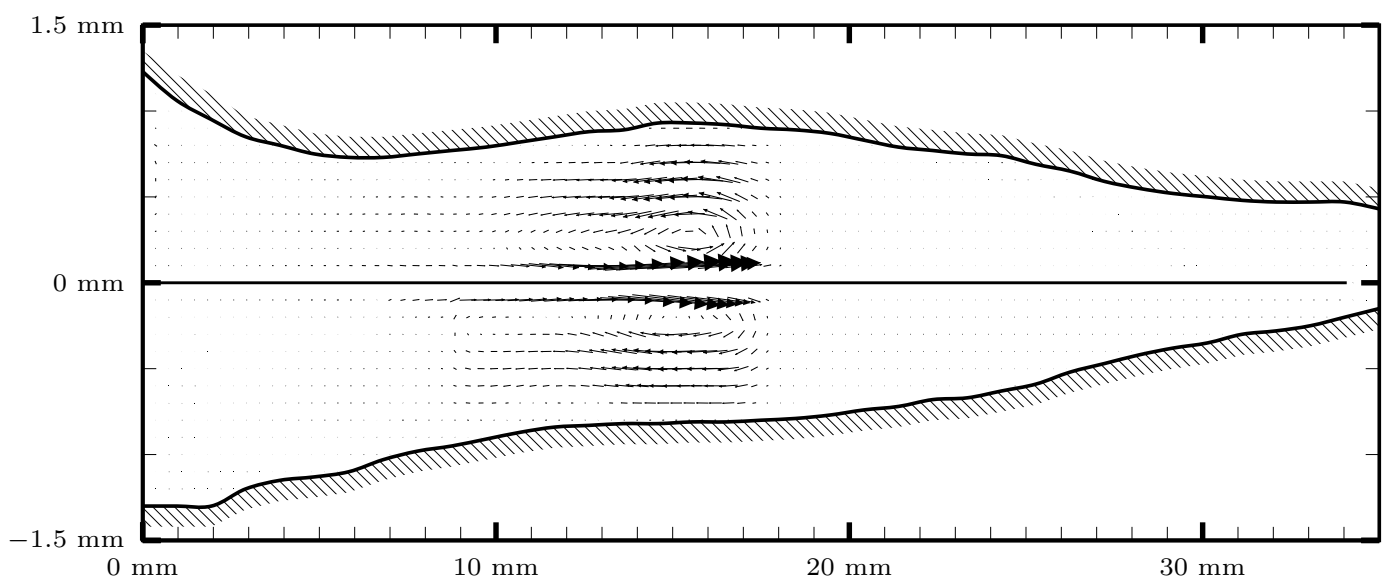

Figure A.26: Lagrangian specification of acousticically driven flows induced by a stimulation frequency of $1024 \mathrm{~Hz}$ in the passive cochlea model. 


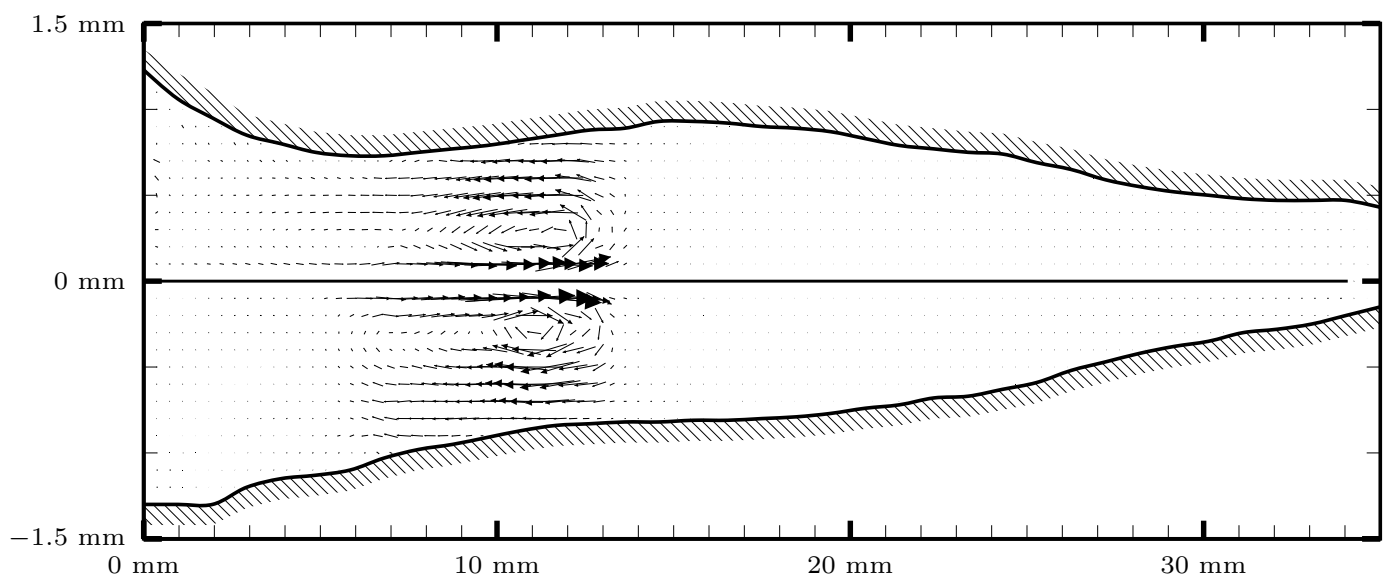

Figure A.27: Lagrangian specification of acousticically driven flows induced by a stimulation frequency of $2048 \mathrm{~Hz}$ in the passive cochlea model.

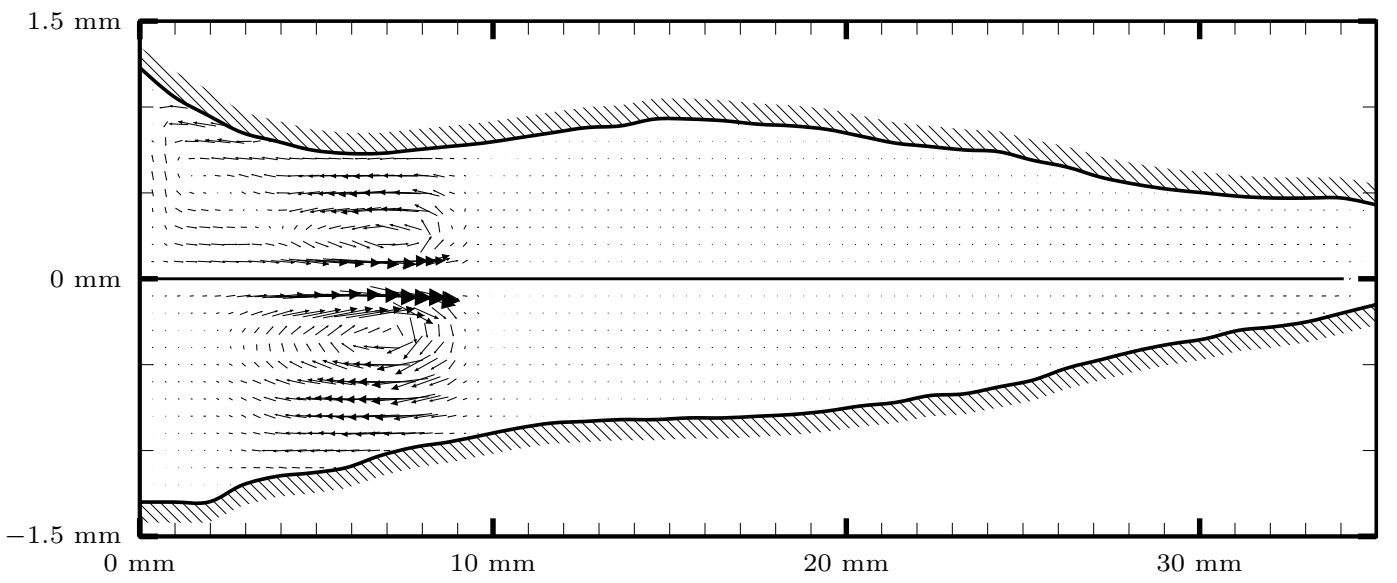

Figure A.28: Lagrangian specification of acousticically driven flows induced by a stimulation frequency of $4096 \mathrm{~Hz}$ in the passive cochlea model. 


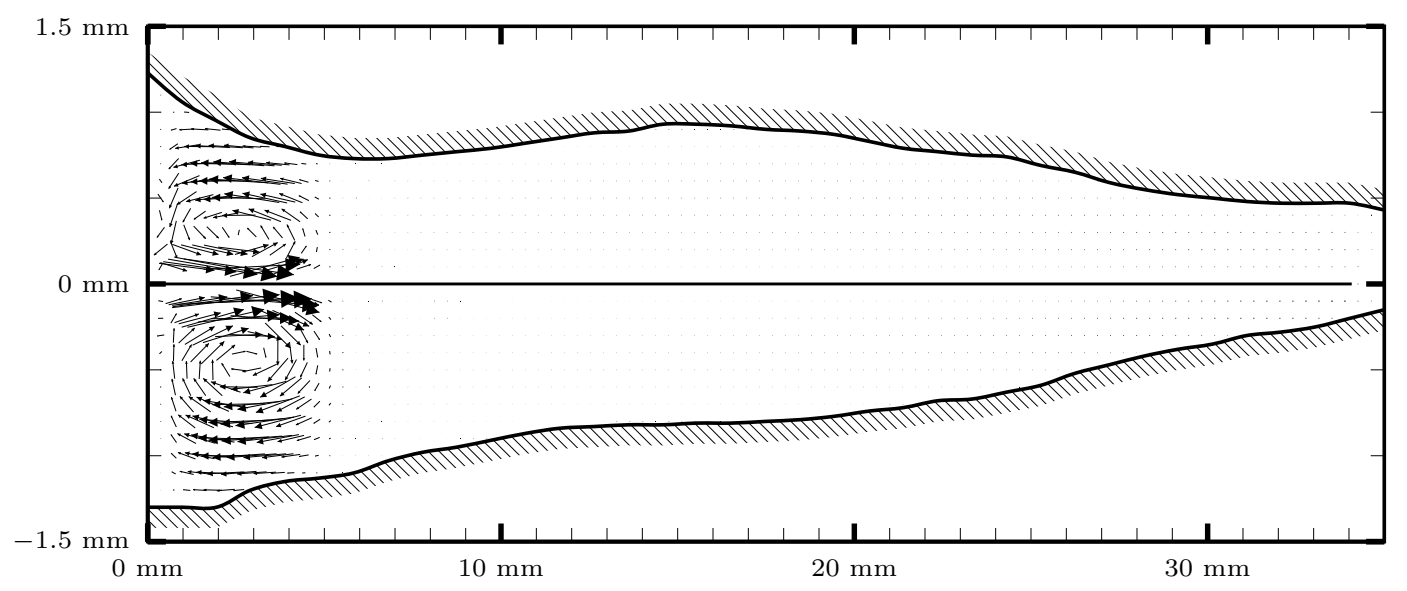

Figure A.29: Lagrangian specification of acousticically driven flows induced by a stimulation frequency of $8192 \mathrm{~Hz}$ in the passive cochlea model. 
Appendix B

Results of the Active Cochlea Model 


\section{B.1 Acoustic Flow Fields at Equilibrium State}

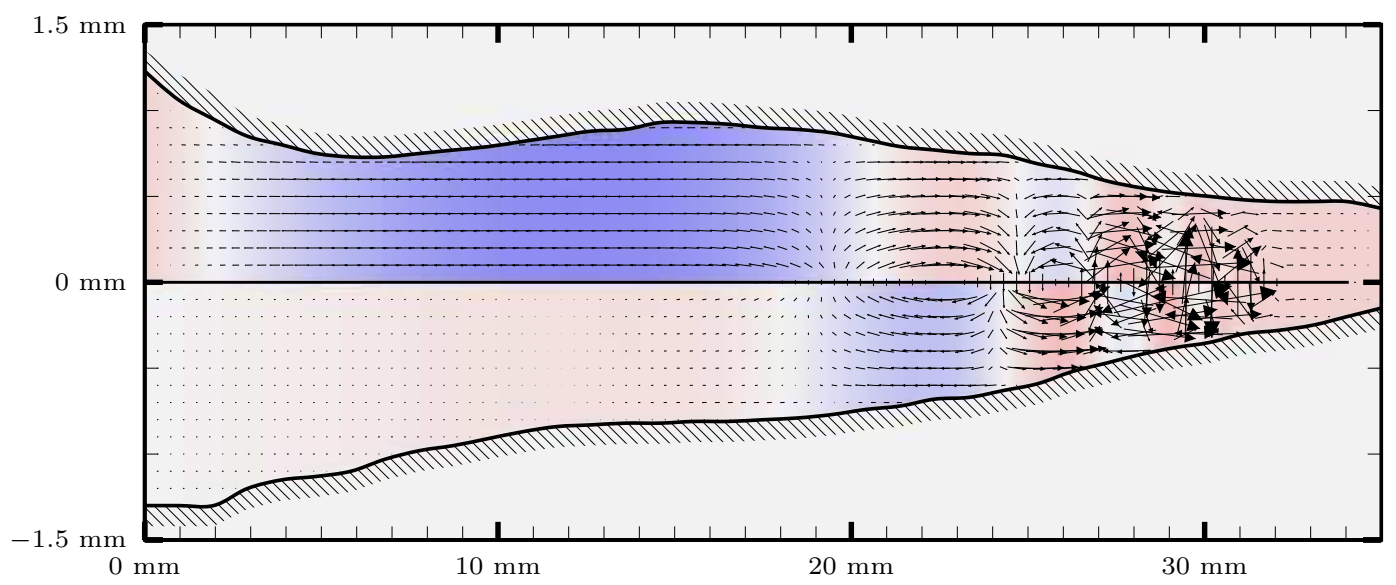

(a)

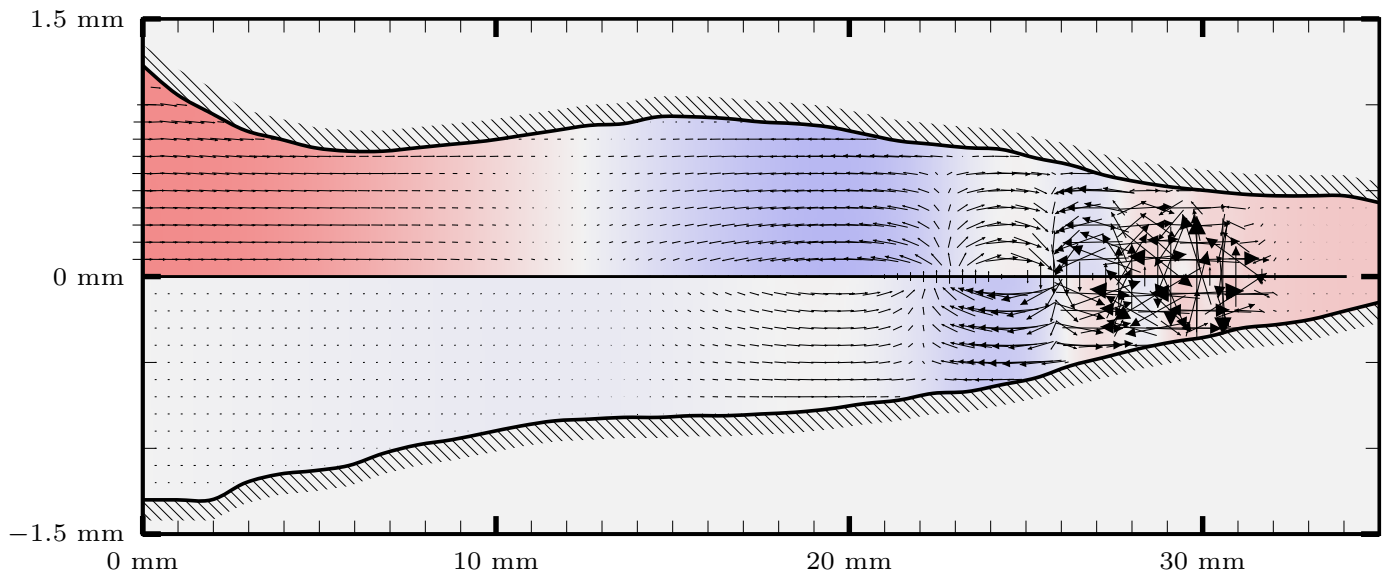

(b)

Figure B.1: Equilibrium state of the first order system at the stimulation frequency of 128Hz in the passive cochlea model. Figure (a) illustrates the real part of the velocity field $\boldsymbol{v}^{\text {(cos) }}$ of the rotary vibration in combination with the real pressure field $p^{(\mathrm{cos})}$. Figure (b) visualizes their imaginary counterparts $\boldsymbol{v}^{\text {(sin) }}$ and $p^{(\mathrm{sin})}$. The intensity of the red (blue) color represents the amount of the positve (negative) pressure. 


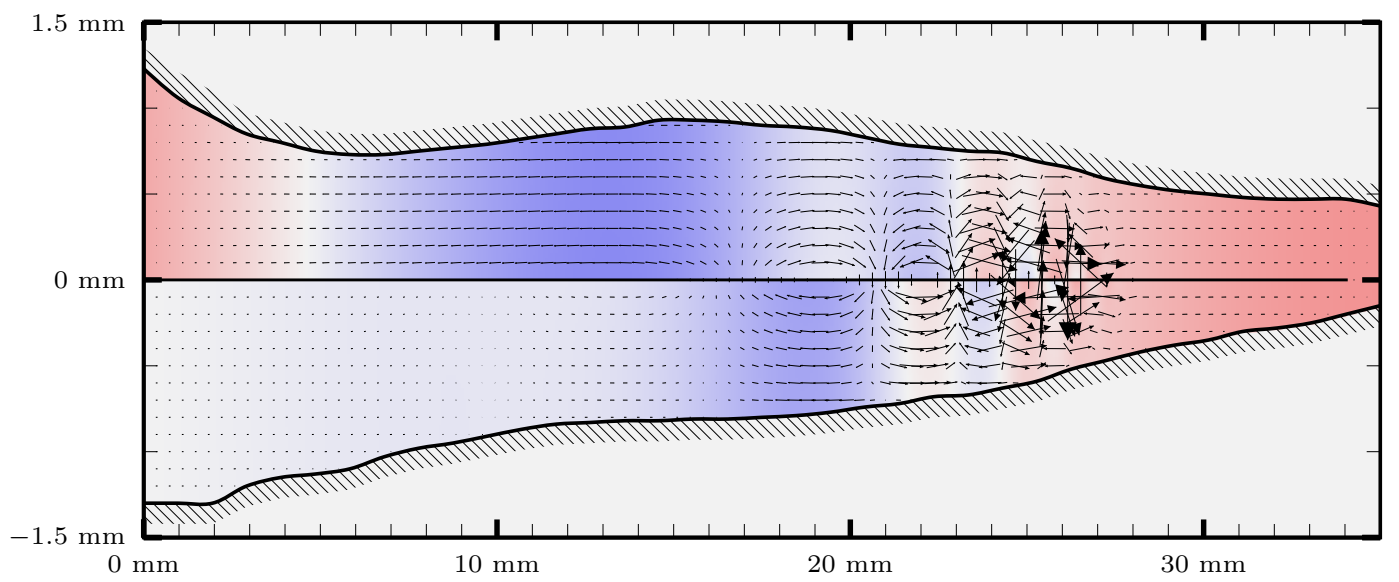

(a)

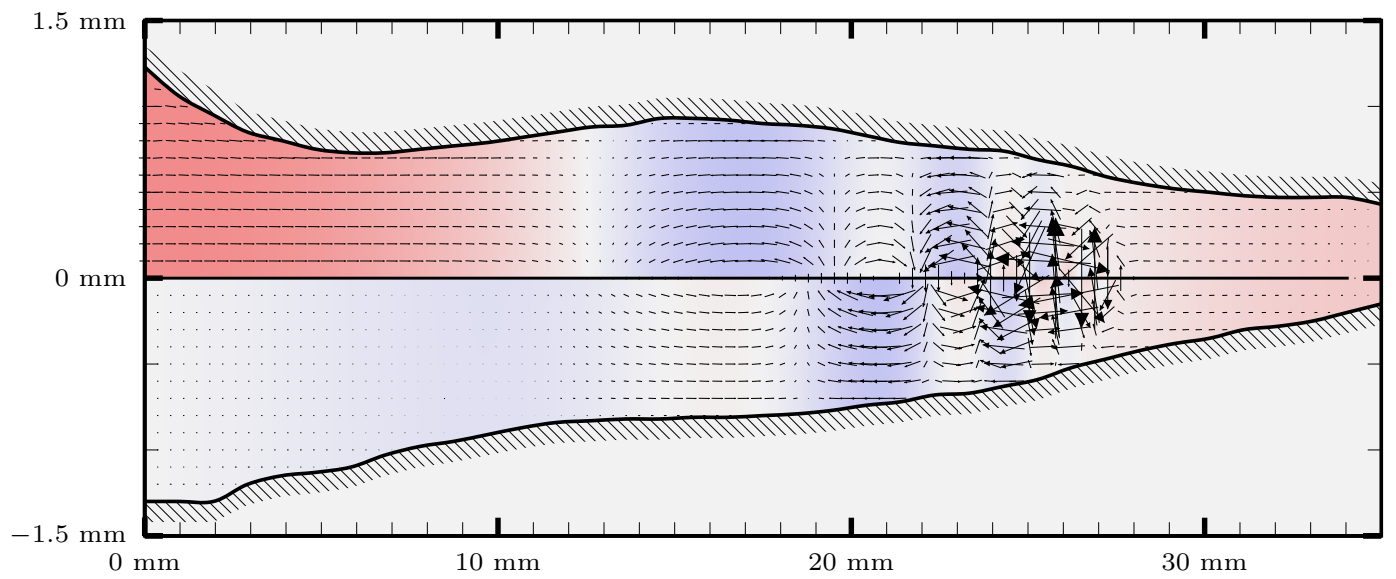

(b)

Figure B.2: Equilibrium state of the first order system at the stimulation frequency of $256 \mathrm{~Hz}$ in the passive cochlea model. Figure (a) illustrates the real part of the velocity field $\boldsymbol{v}^{\text {(cos) }}$ of the rotary vibration in combination with the real pressure field $p^{(\mathrm{cos})}$. Figure (b) visualizes their imaginary counterparts $\boldsymbol{v}^{(\mathrm{sin})}$ and $p^{(\sin )}$. The intensity of the red (blue) color represents the amount of the positve (negative) pressure. 


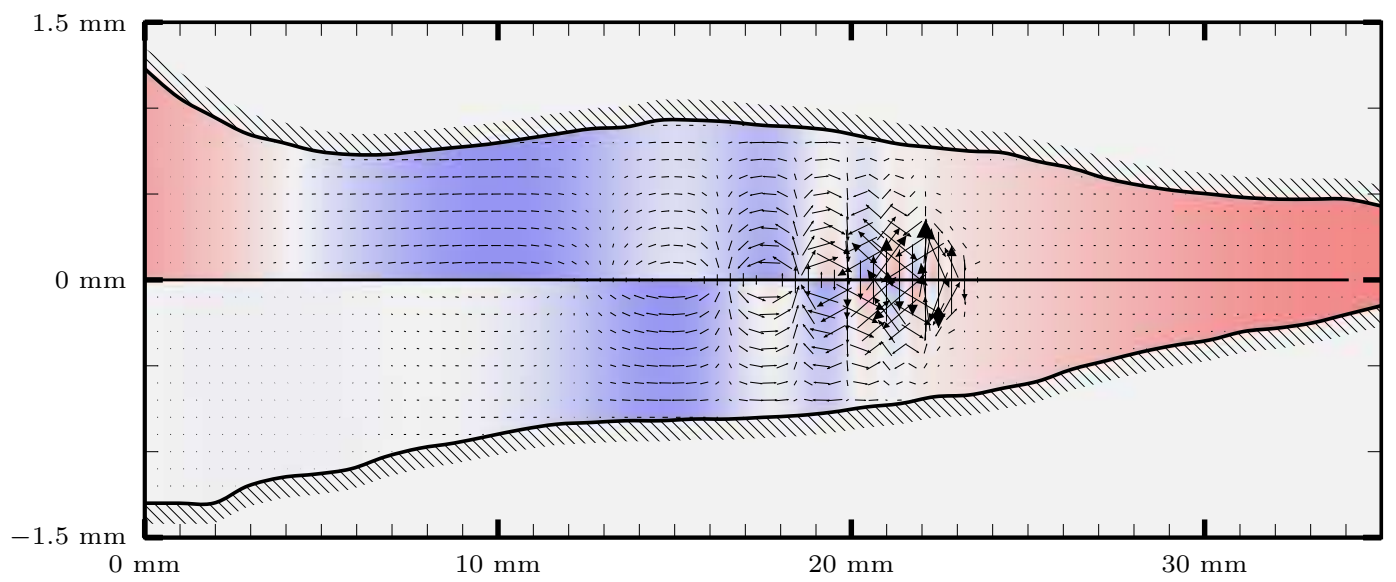

(a)

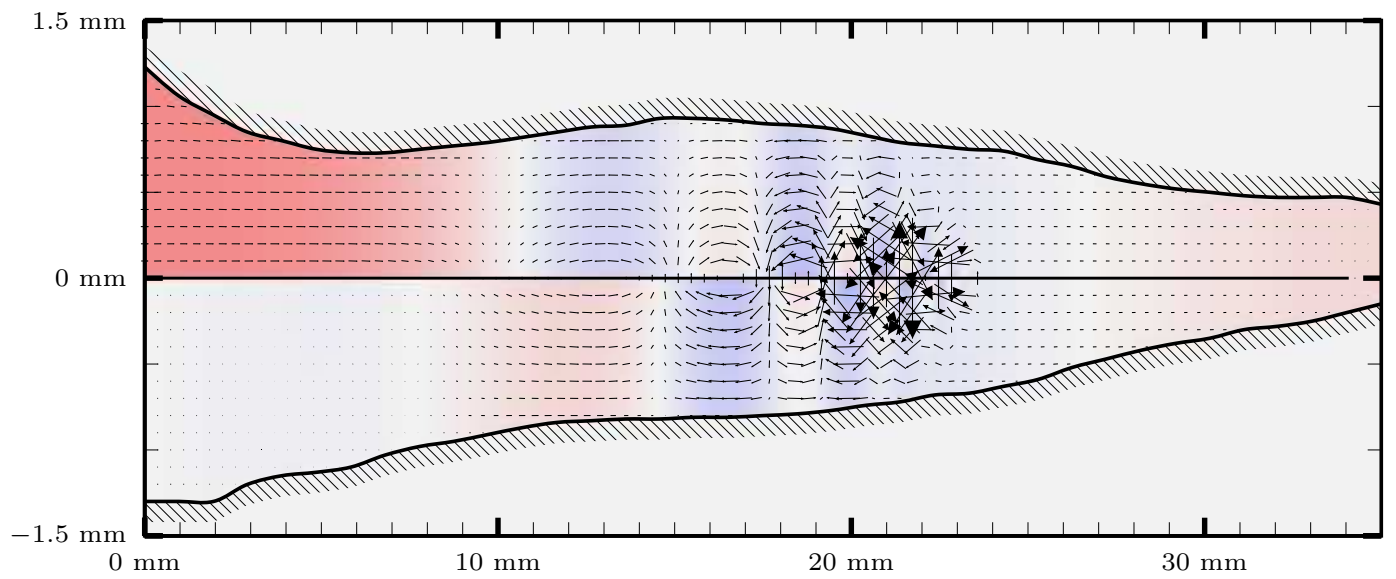

(b)

Figure B.3: Equilibrium state of the first order system at the stimulation frequency of 512Hz in the passive cochlea model. Figure (a) illustrates the real part of the velocity field $\boldsymbol{v}^{\text {(cos) }}$ of the rotary vibration in combination with the real pressure field $p^{(\mathrm{cos})}$. Figure (b) visualizes their imaginary counterparts $\boldsymbol{v}^{(\mathrm{sin})}$ and $p^{(\sin )}$. The intensity of the red (blue) color represents the amount of the positve (negative) pressure. 


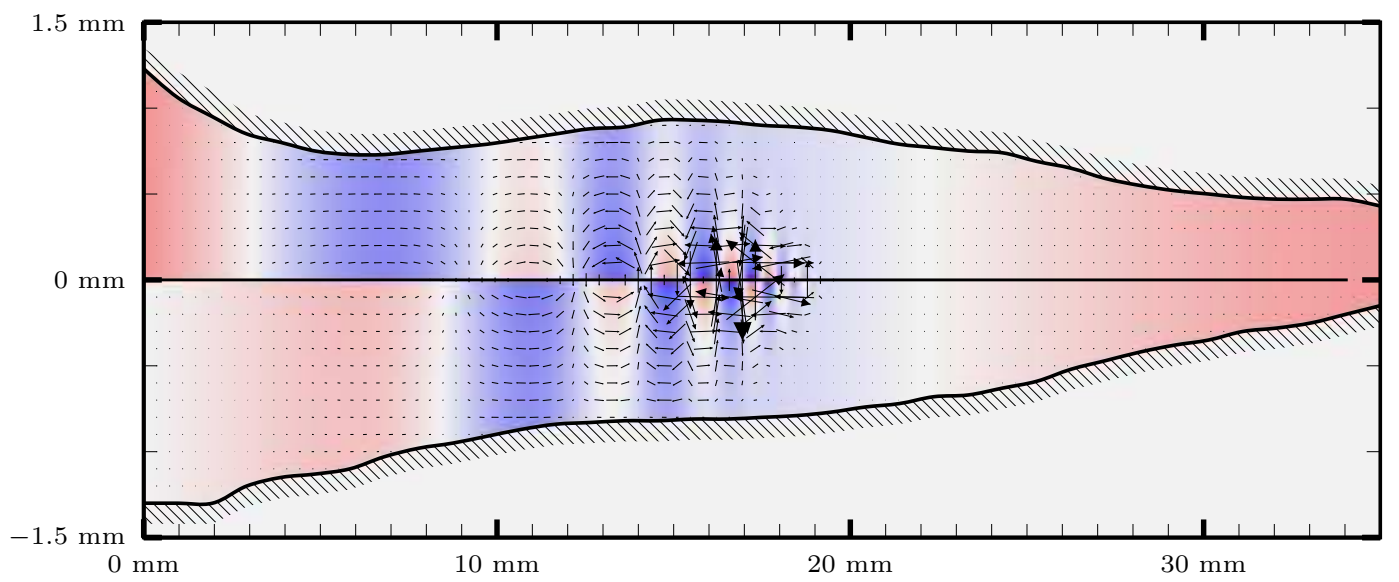

(a)

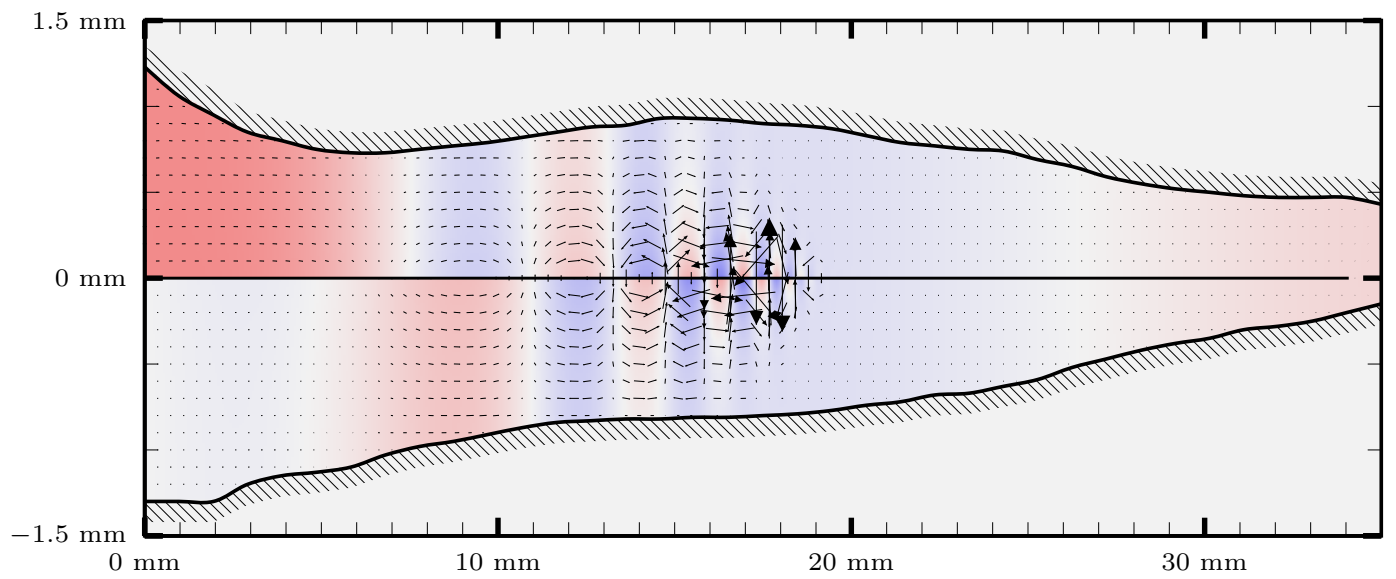

(b)

Figure B.4: Equilibrium state of the first order system at the stimulation frequency of 1024Hz in the passive cochlea model. Figure (a) illustrates the real part of the velocity field $\boldsymbol{v}^{\text {(cos) }}$ of the rotary vibration in combination with the real pressure field $p^{(\mathrm{cos})}$. Figure (b) visualizes their imaginary counterparts $\boldsymbol{v}^{(\mathrm{sin})}$ and $p^{(\sin )}$. The intensity of the red (blue) color represents the amount of the positve (negative) pressure. 


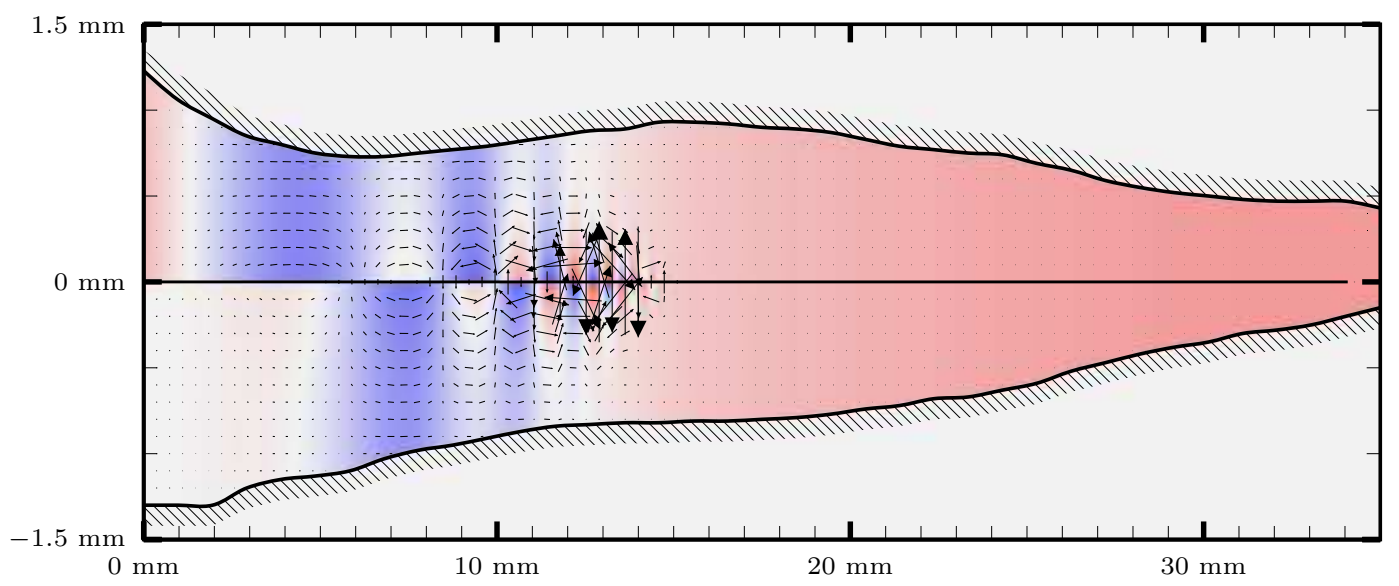

(a)

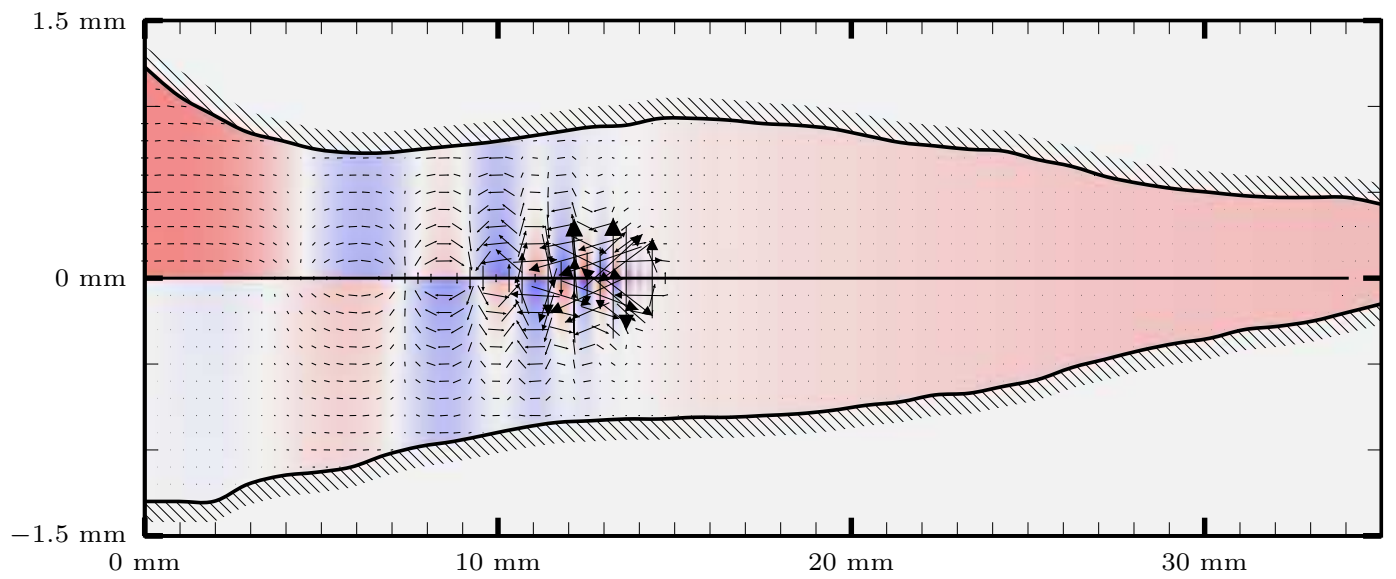

(b)

Figure B.5: Equilibrium state of the first order system at the stimulation frequency of $2048 \mathrm{~Hz}$ in the passive cochlea model. Figure (a) illustrates the real part of the velocity field $\boldsymbol{v}^{\text {(cos) }}$ of the rotary vibration in combination with the real pressure field $p^{(\mathrm{cos})}$. Figure (b) visualizes their imaginary counterparts $\boldsymbol{v}^{(\mathrm{sin})}$ and $p^{(\sin )}$. The intensity of the red (blue) color represents the amount of the positve (negative) pressure. 


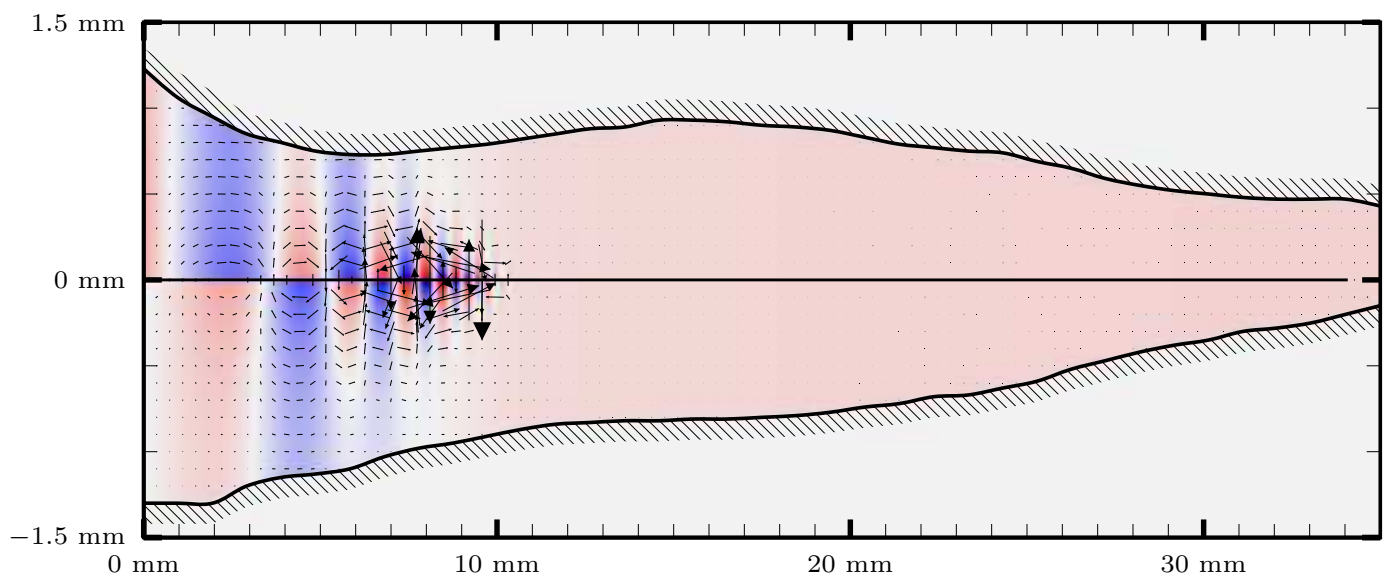

(a)

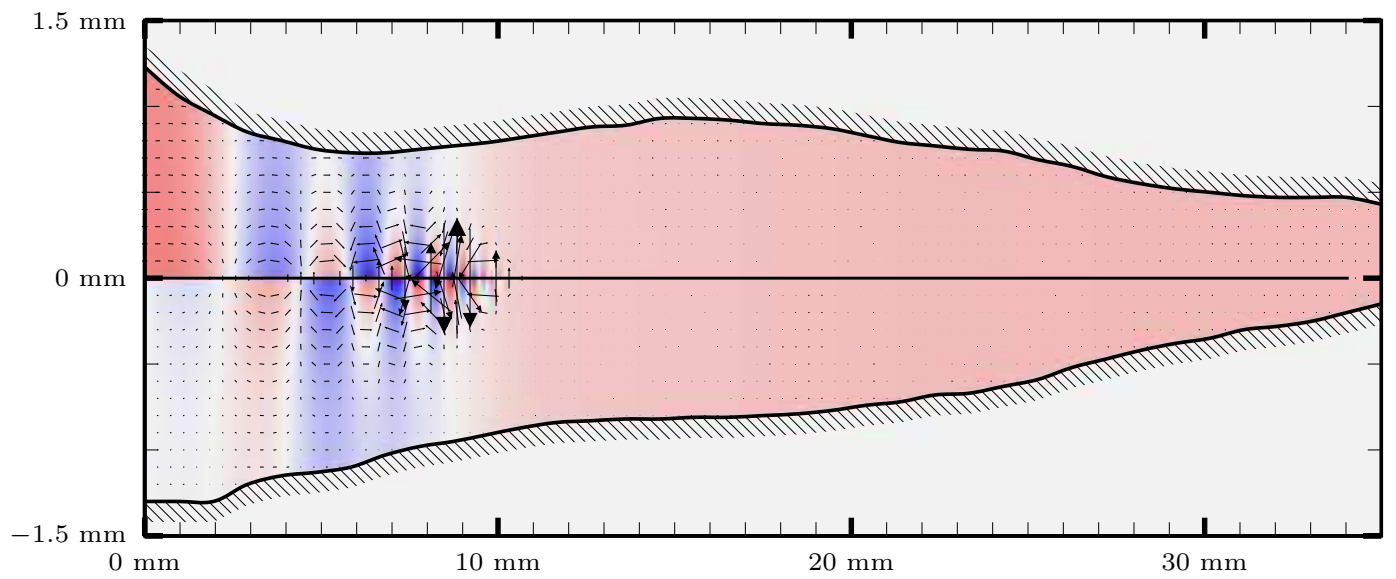

(b)

Figure B.6: Equilibrium state of the first order system at the stimulation frequency of $4096 \mathrm{~Hz}$ in the passive cochlea model. Figure (a) illustrates the real part of the velocity field $\boldsymbol{v}^{\text {(cos) }}$ of the rotary vibration in combination with the real pressure field $p^{(\mathrm{cos})}$. Figure (b) visualizes their imaginary counterparts $\boldsymbol{v}^{(\mathrm{sin})}$ and $p^{(\sin )}$. The intensity of the red (blue) color represents the amount of the positve (negative) pressure. 


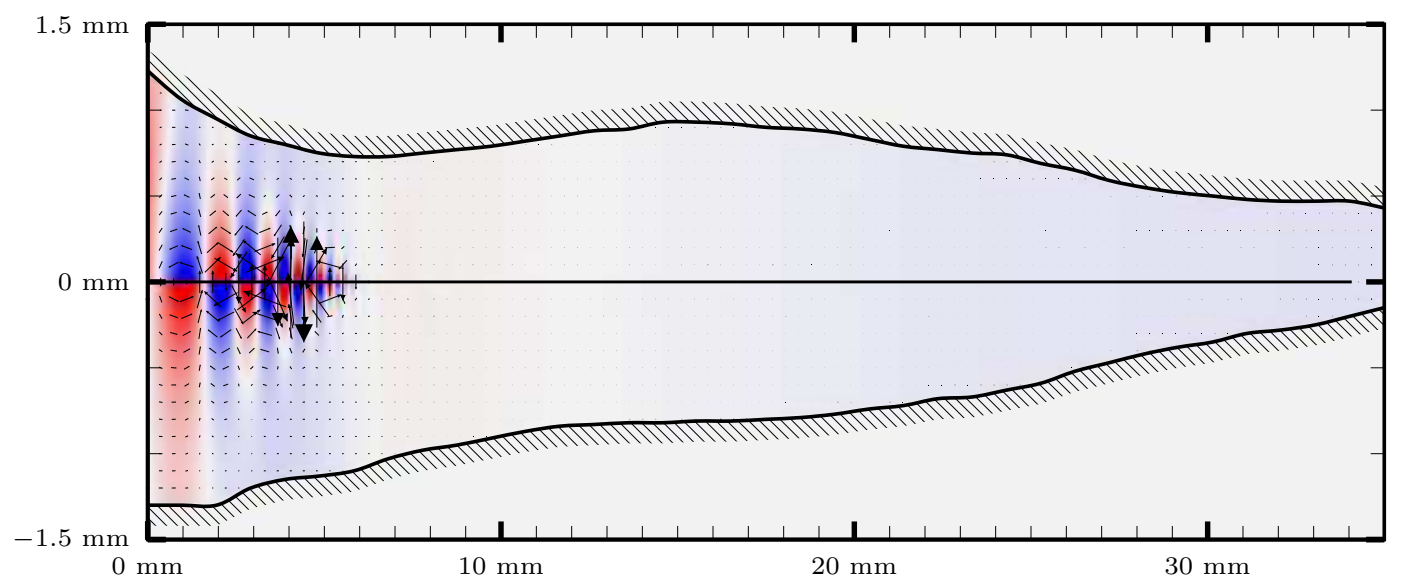

(a)

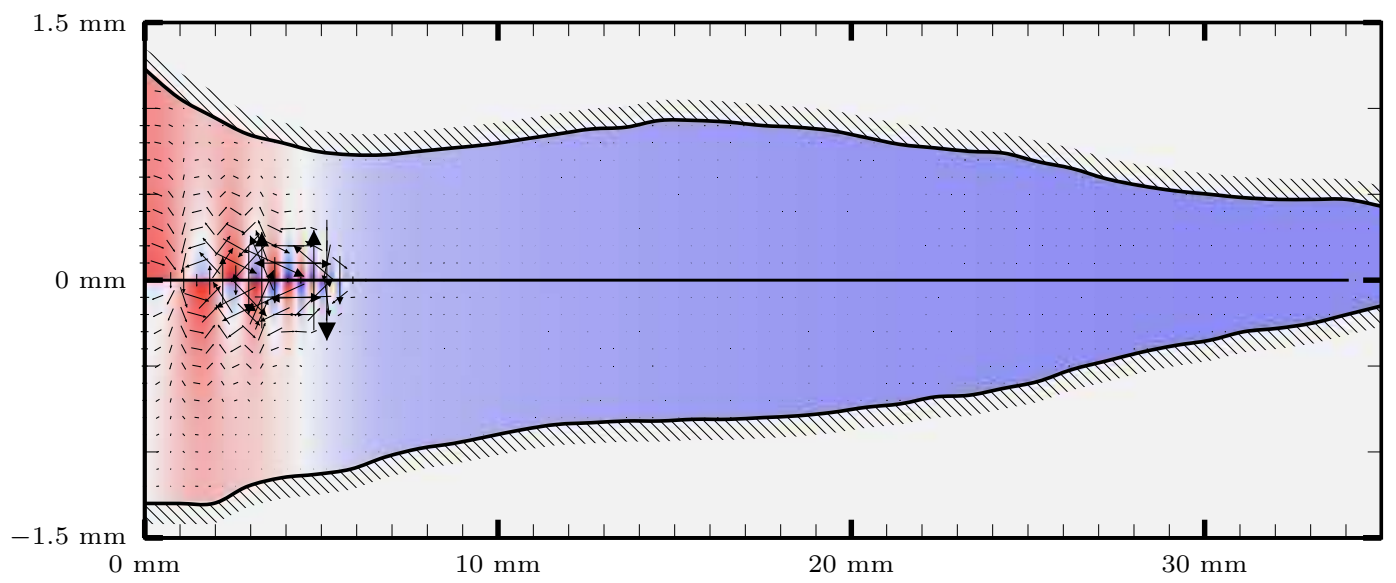

(b)

Figure B.7: Equilibrium state of the first order system at the stimulation frequency of 8192Hz in the passive cochlea model. Figure (a) illustrates the real part of the velocity field $\boldsymbol{v}^{(\mathrm{cos})}$ of the rotary vibration in combination with the real pressure field $p^{(\mathrm{cos})}$. Figure (b) visualizes their imaginary counterparts $\boldsymbol{v}^{(\mathrm{sin})}$ and $p^{(\sin )}$. The intensity of the red (blue) color represents the amount of the positve (negative) pressure. 


\section{B.2 Displacement of the Basilar Membrane}

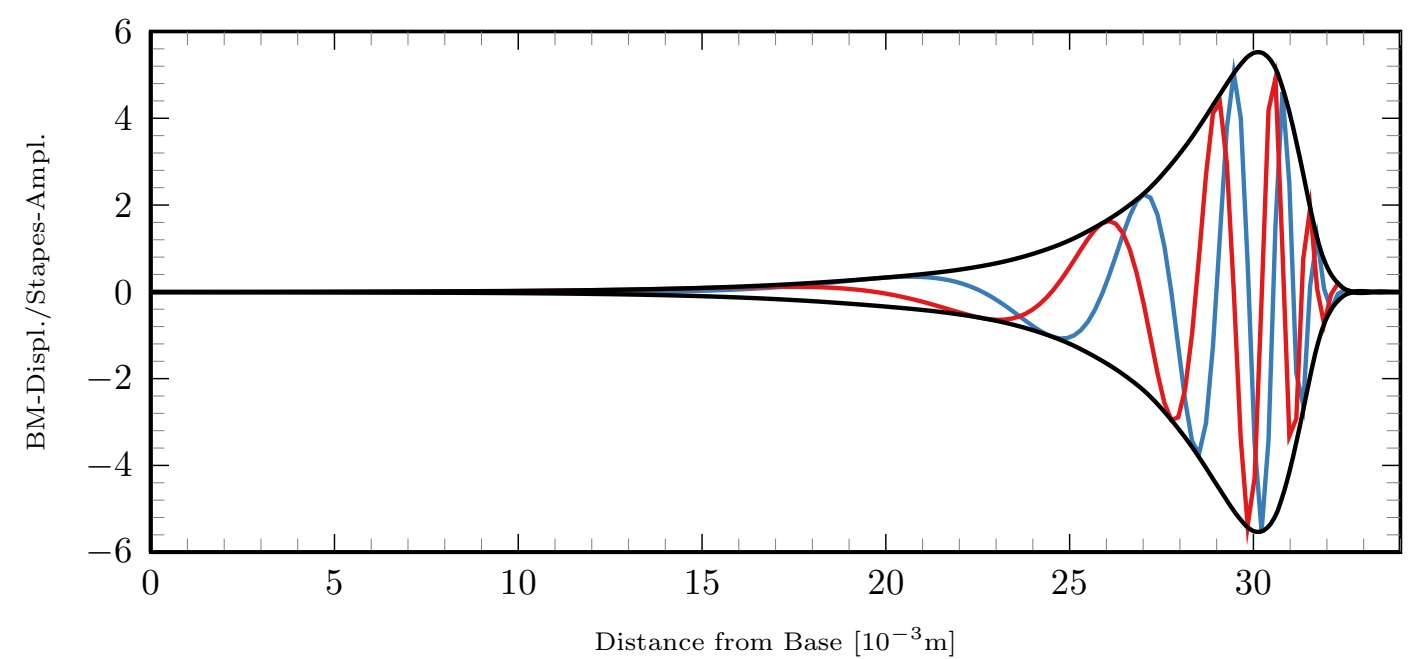

Figure B.8: Traveling wave motion of the basilar membrane at the stimulation frequency of $128 \mathrm{~Hz}$ in the active cochlea model. The red line represents the cosine part $\xi^{(\mathrm{cos})}$ of the oscillatory motion, whereas the blue line is associated with the sine component $\xi^{(\sin )}$. The black line illustrates the envelope. 


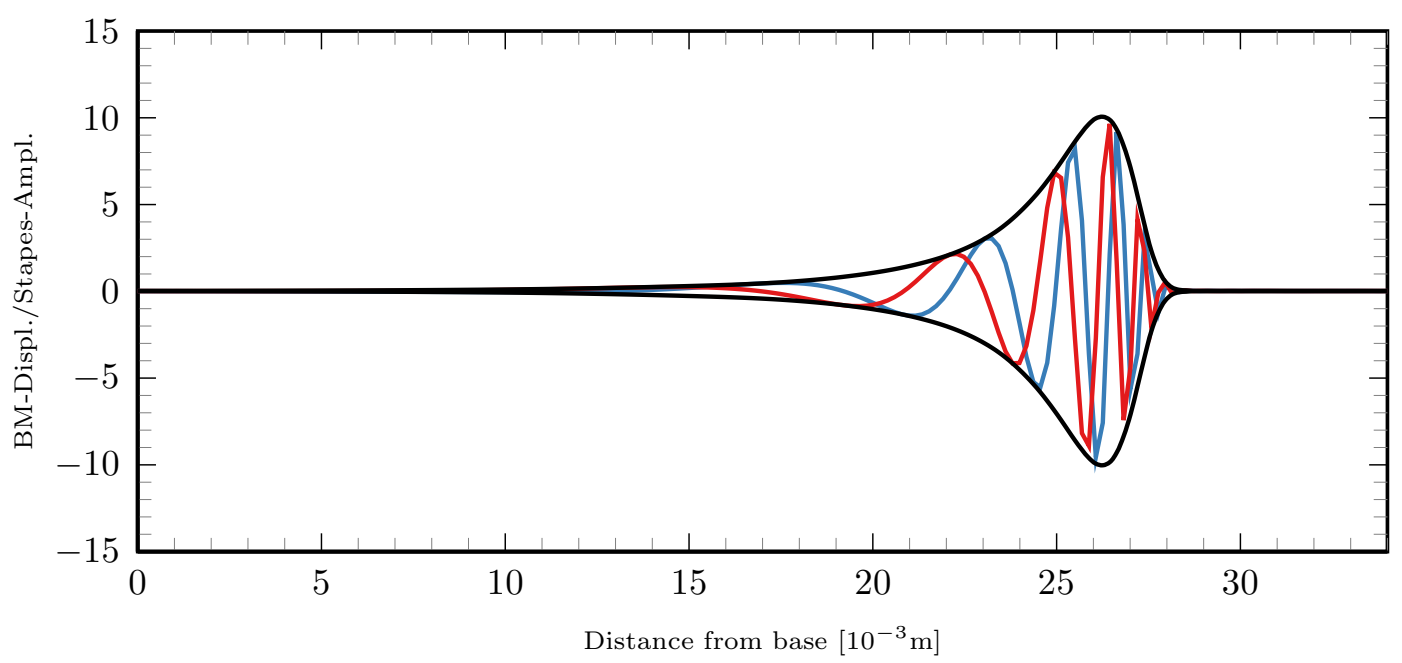

Figure B.9: Traveling wave motion of the basilar membrane at the stimulation frequency of $256 \mathrm{~Hz}$ in the active cochlea model. The red line represents the cosine part $\xi^{(\mathrm{cos})}$ of the oscillatory motion, whereas the blue line is associated with the sine component $\xi^{(\sin )}$. The black line illustrates the envelope.

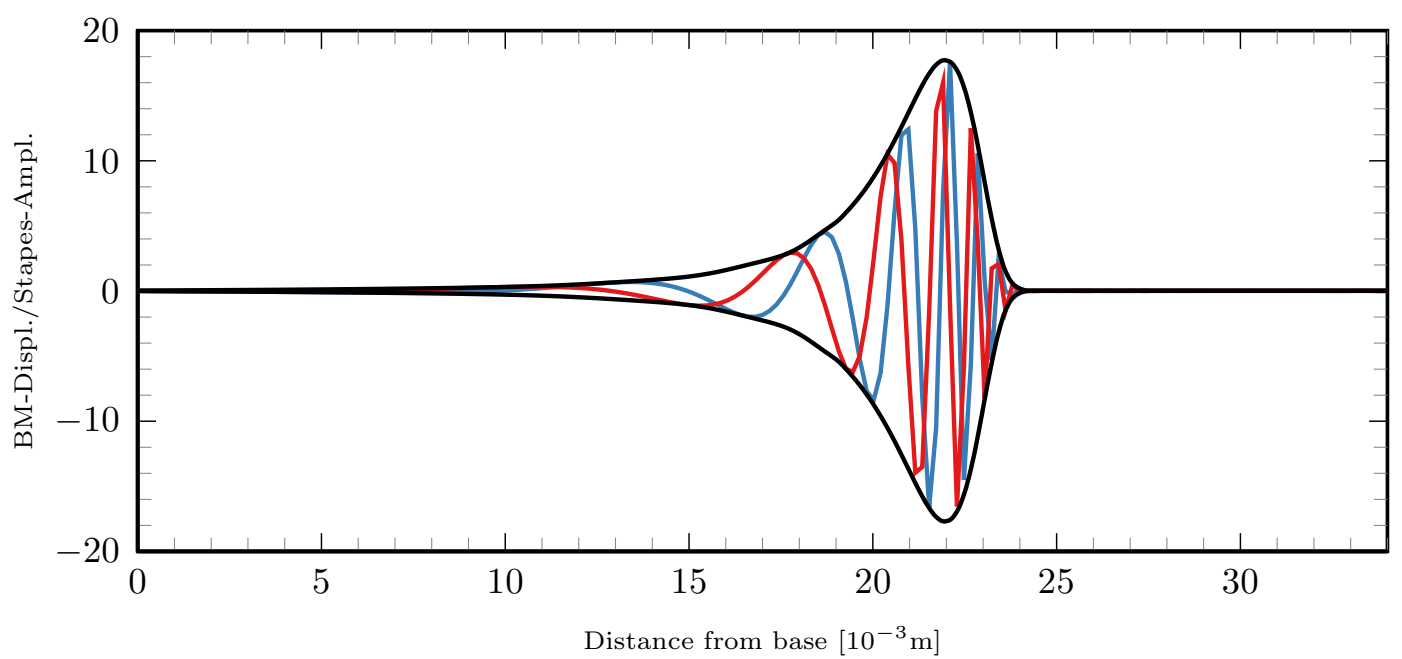

Figure B.10: Traveling wave motion of the basilar membrane at the stimulation frequency of 512Hz in the active cochlea model. The red line represents the cosine part $\xi^{(\cos )}$ of the oscillatory motion, whereas the blue line is associated with the sine component $\xi^{(\sin )}$. The black line illustrates the envelope. 


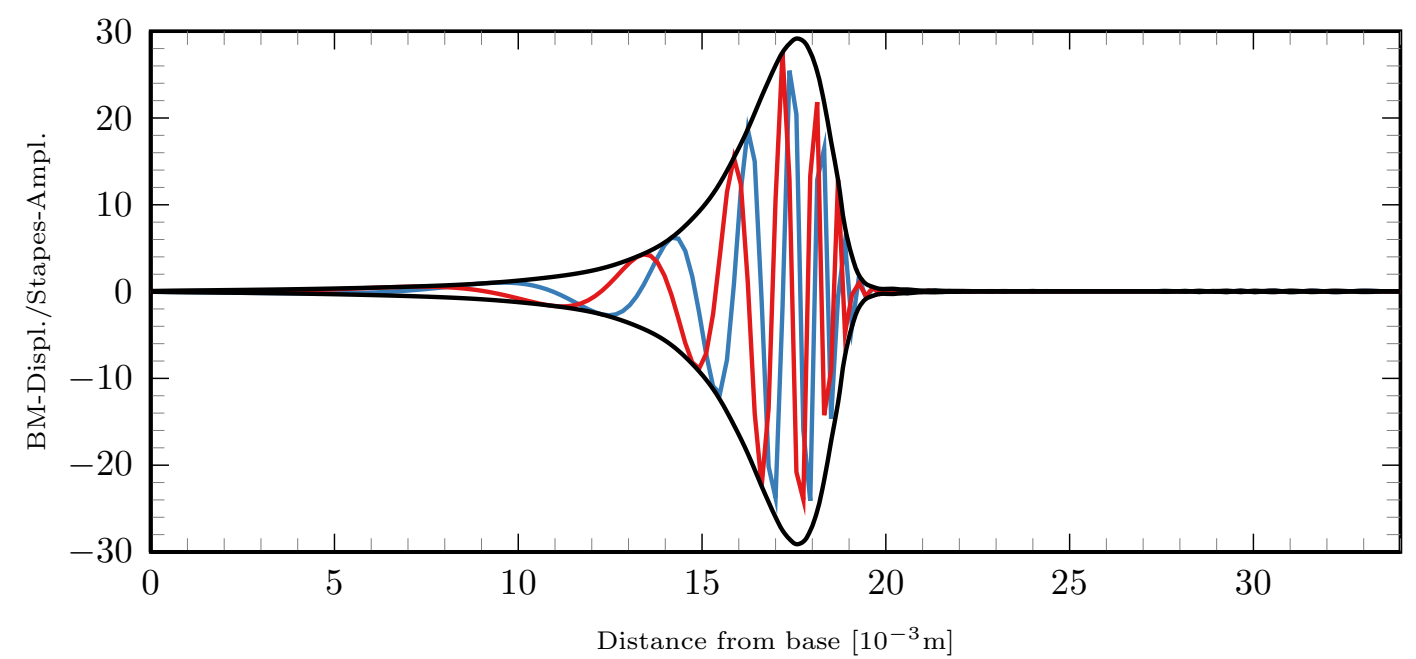

Figure B.11: Traveling wave motion of the basilar membrane at the stimulation frequency of 1024Hz in the active cochlea model. The red line represents the cosine part $\xi^{(\mathrm{cos})}$ of the oscillatory motion, whereas the blue line is associated with the sine component $\xi^{(\sin )}$. The black line illustrates the envelope.

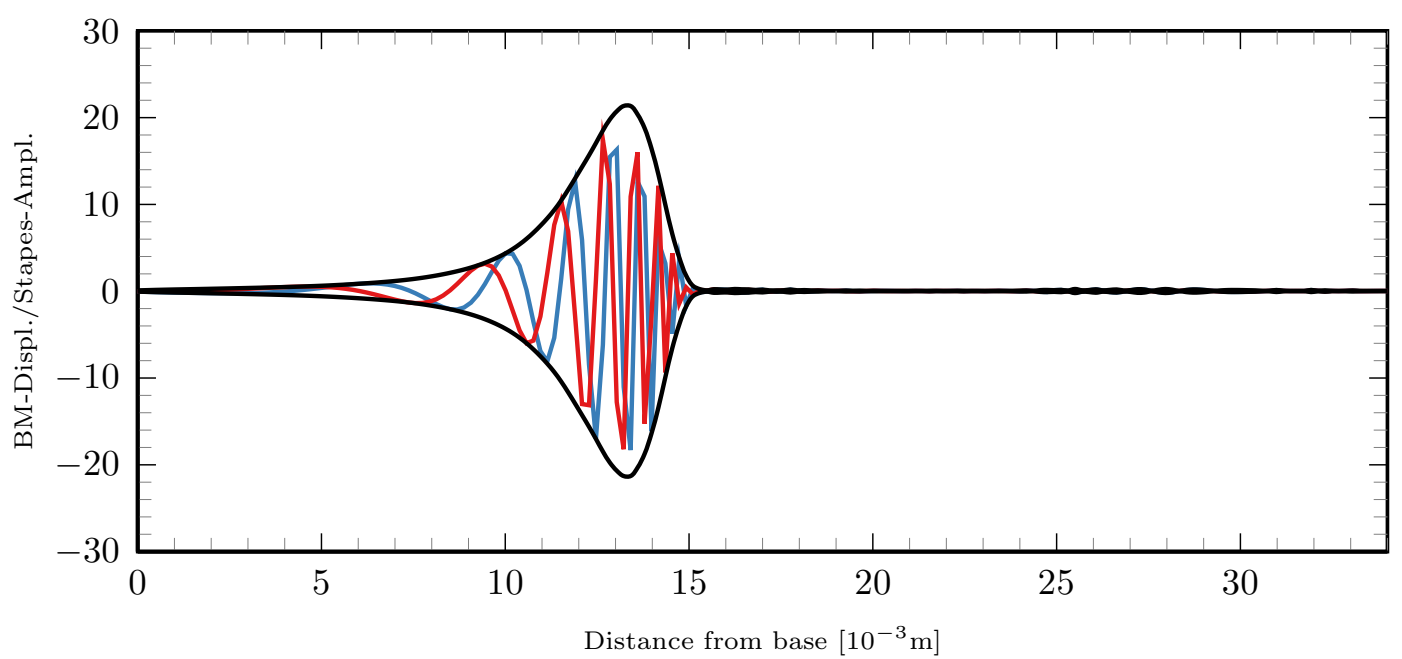

Figure B.12: Traveling wave motion of the basilar membrane at the stimulation frequency of $2048 \mathrm{~Hz}$ in the active cochlea model. The red line represents the cosine part $\xi^{(\mathrm{cos})}$ of the oscillatory motion, whereas the blue line is associated with the sine component $\xi^{(\sin )}$. The black line illustrates the envelope. 


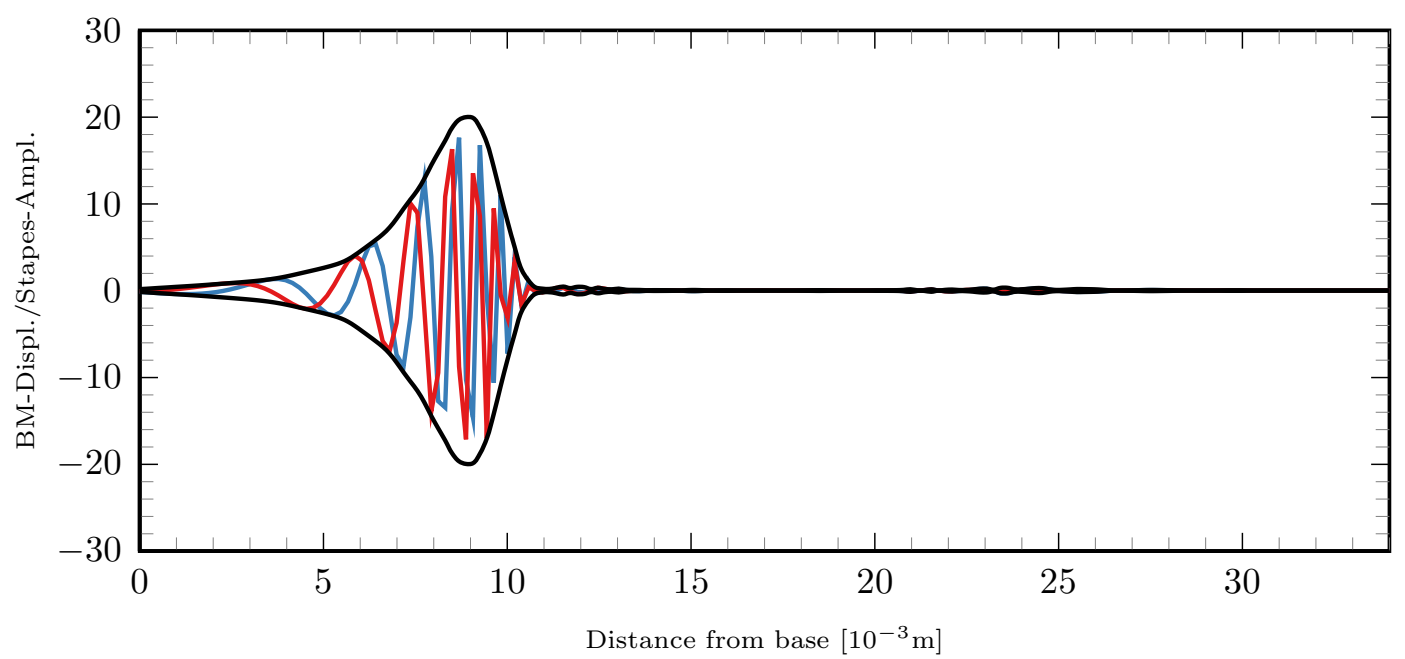

Figure B.13: Traveling wave motion of the basilar membrane at the stimulation frequency of $4096 \mathrm{~Hz}$ in the active cochlea model. The red line represents the cosine part $\xi^{(\mathrm{cos})}$ of the oscillatory motion, whereas the blue line is associated with the sine component $\xi^{(\sin )}$. The black line illustrates the envelope.

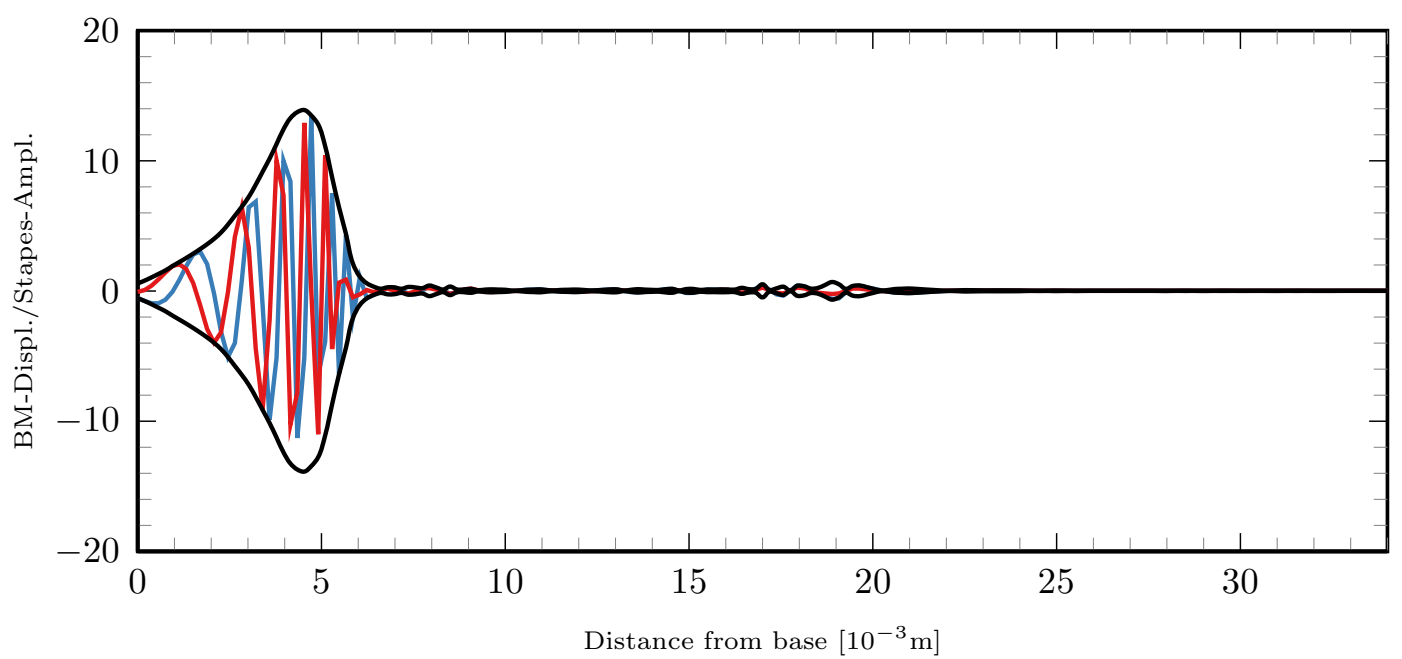

Figure B.14: Traveling wave motion of the basilar membrane at the stimulation frequency of 8192Hz in the active cochlea model. The red line represents the cosine part $\xi^{(\mathrm{cos})}$ of the oscillatory motion, whereas the blue line is associated with the sine component $\xi^{(\sin )}$. The black line illustrates the envelope. 


\section{B.3 Acoustic Streaming Flow Field}

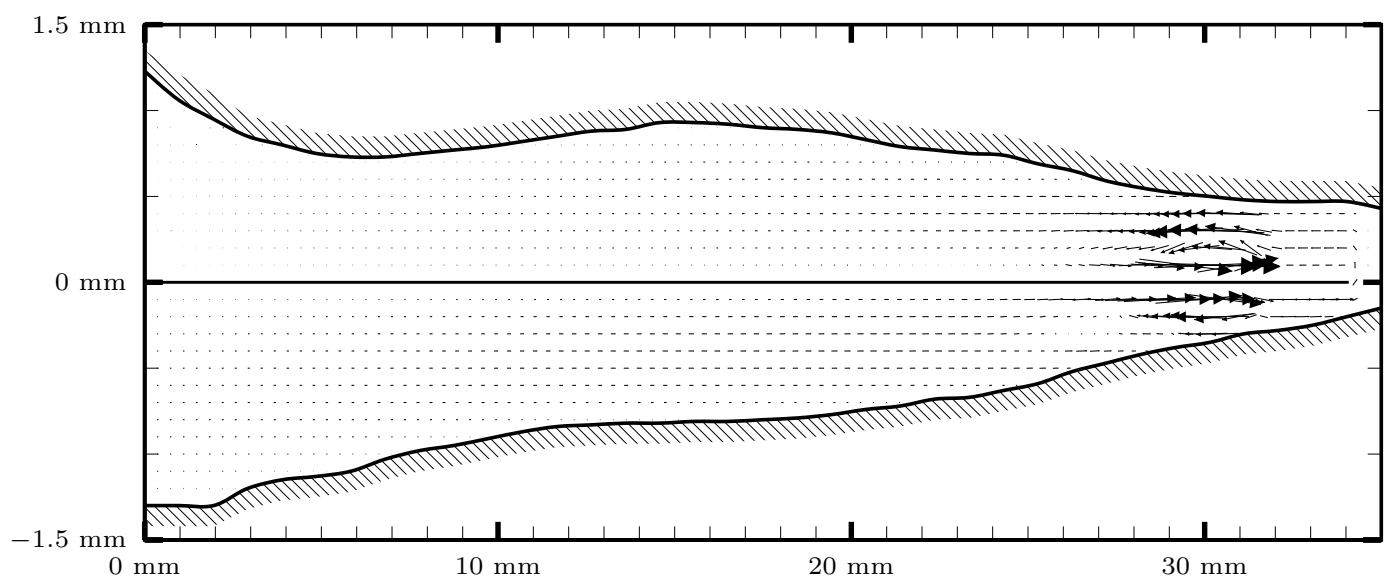

Figure B.15: Lagrangian specification of acousticically driven flows induced by a stimulation frequency of $128 \mathrm{~Hz}$ in the active cochlea model.

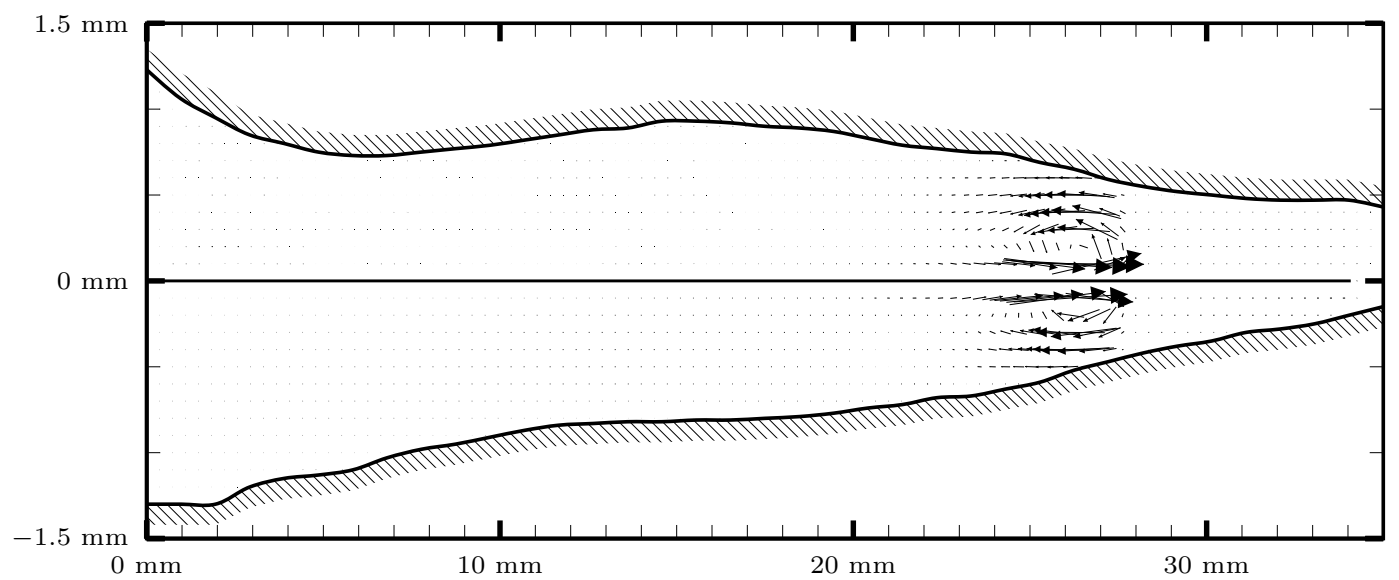

Figure B.16: Lagrangian specification of acousticically driven flows induced by a stimulation frequency of $256 \mathrm{~Hz}$ in the active cochlea model. 


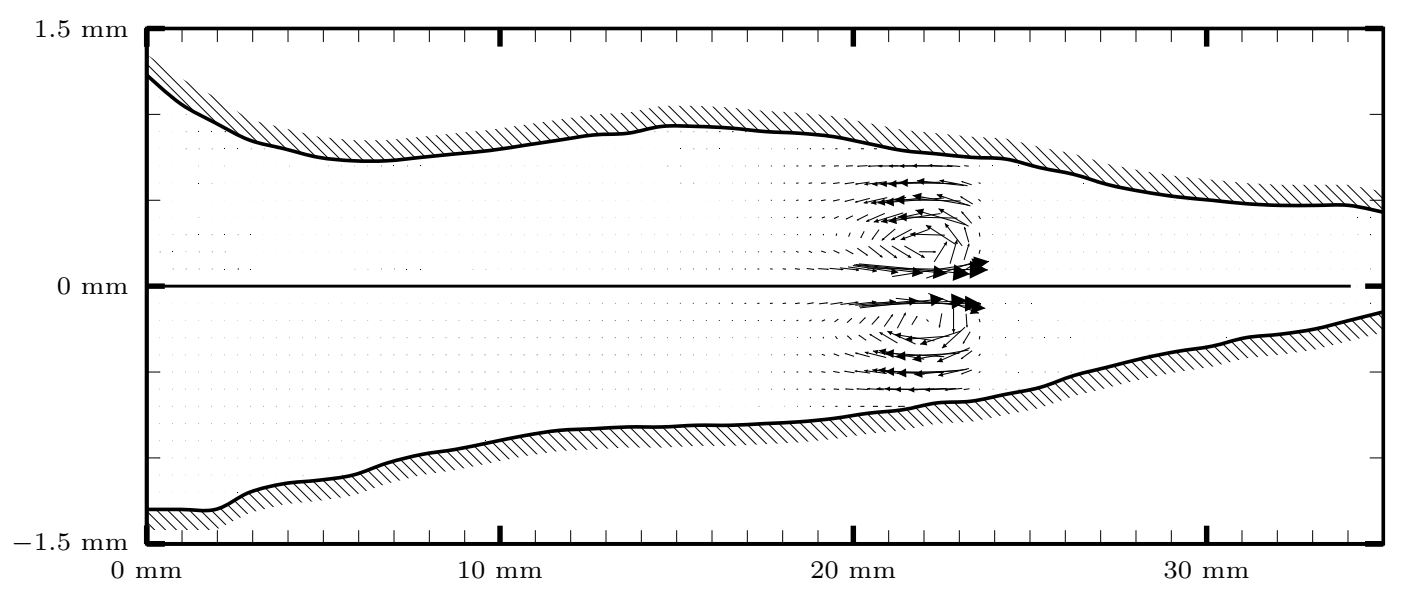

Figure B.17: Lagrangian specification of acousticically driven flows induced by a stimulation frequency of $512 \mathrm{~Hz}$ in the active cochlea model.

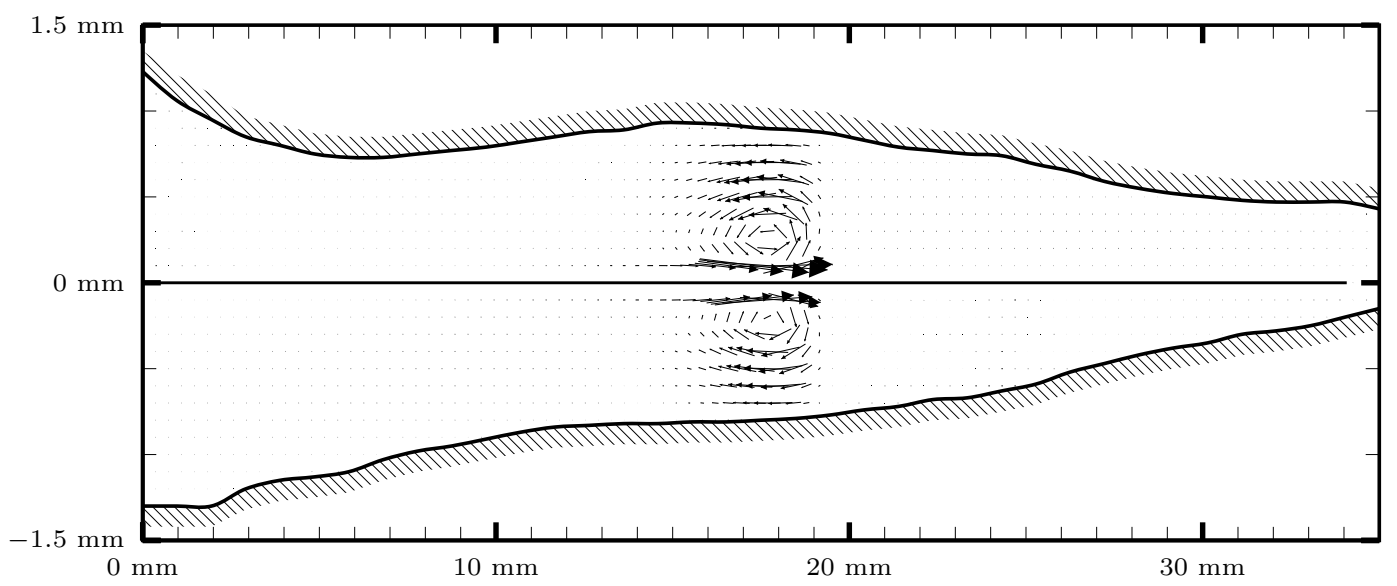

Figure B.18: Lagrangian specification of acousticically driven flows induced by a stimulation frequency of $1024 \mathrm{~Hz}$ in the active cochlea model. 


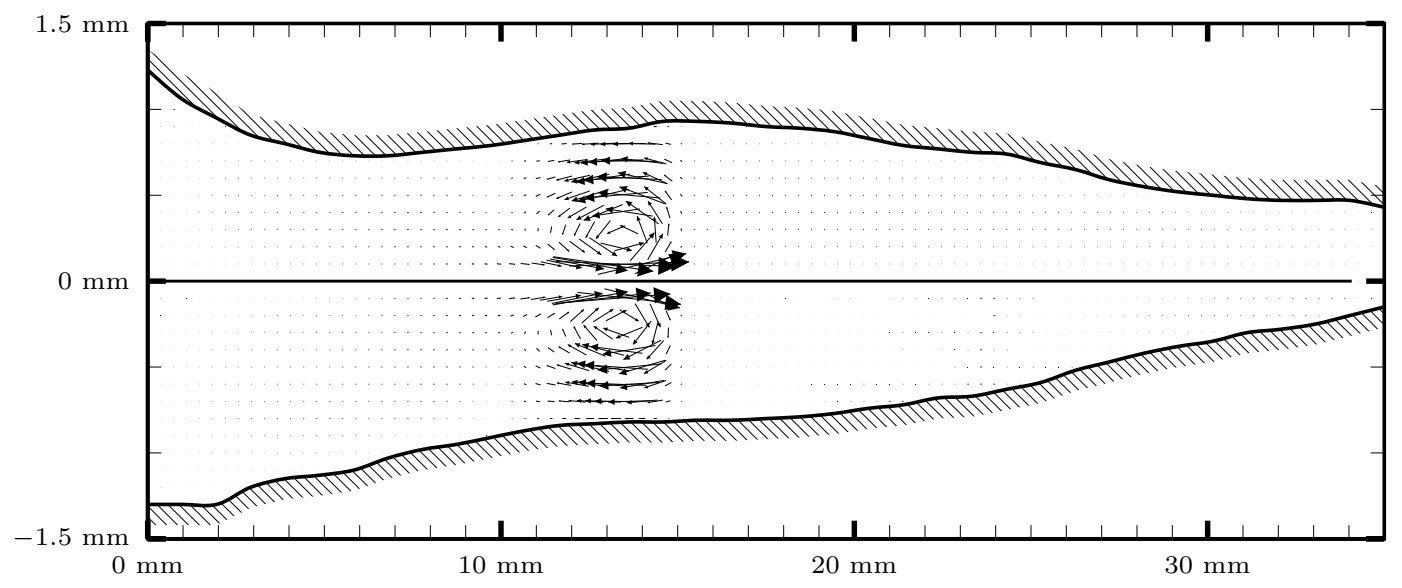

Figure B.19: Lagrangian specification of acousticically driven flows induced by a stimulation frequency of $2048 \mathrm{~Hz}$ in the active cochlea model.

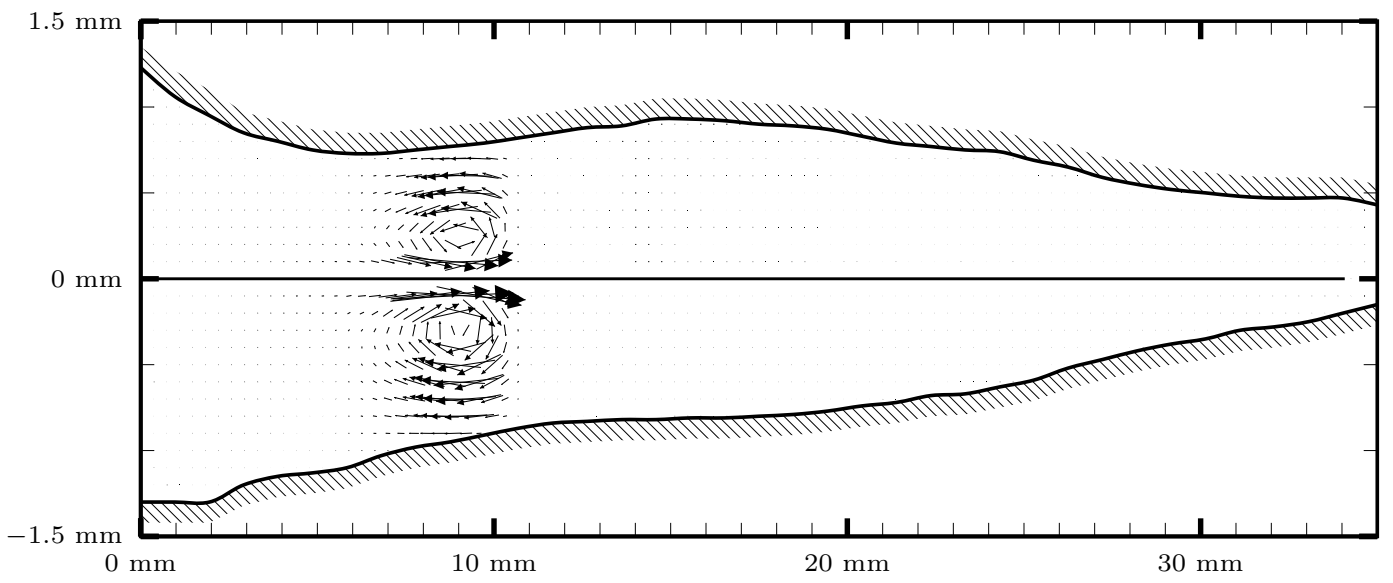

Figure B.20: Lagrangian specification of acousticically driven flows induced by a stimulation frequency of $4096 \mathrm{~Hz}$ in the active cochlea model. 


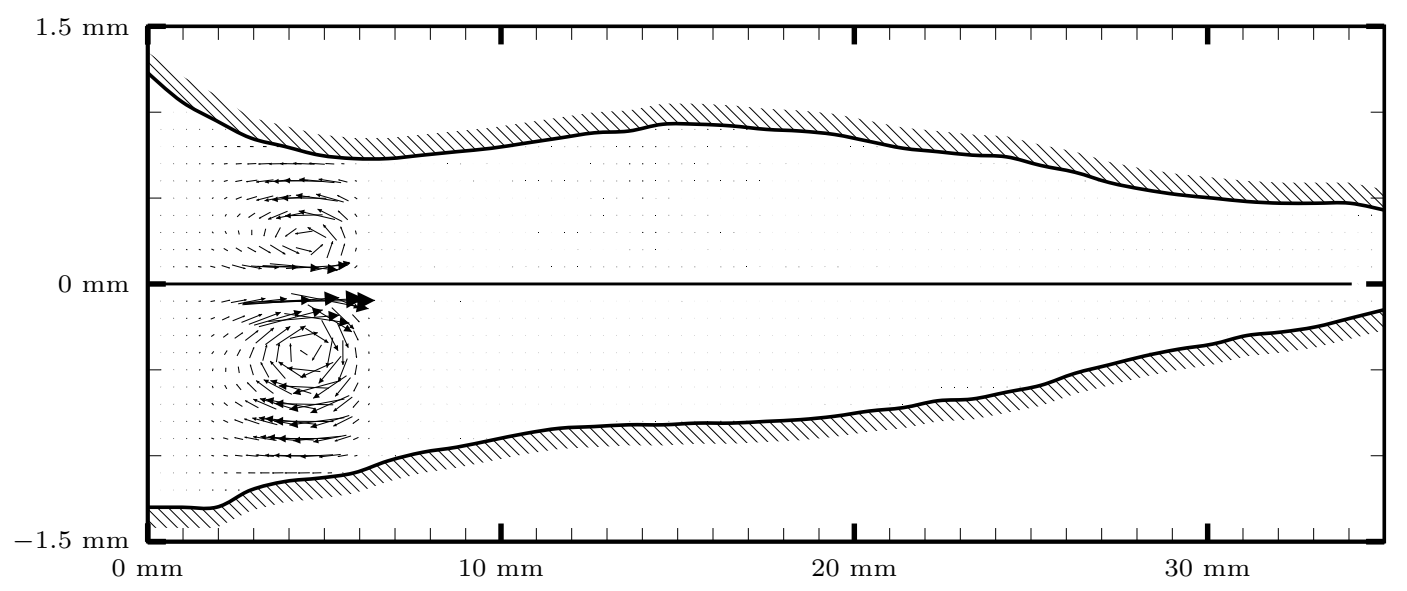

Figure B.21: Lagrangian specification of acousticically driven flows induced by a stimulation frequency of $8192 \mathrm{~Hz}$ in the active cochlea model. 


\section{List of Figures}

1.1 Structure of the human ear. . . . . . . . . . . . . . 2

1.2 Structure of the cohlea. . . . . . . . . . . . . . . . . 3

1.3 Schematic layout of the computational model. . . . . . . . . . . . . 4

1.4 Principal process of the model development. . . . . . . . . . . . 6

2.1 The shape of the two-dimensional cochlea model. . . . . . . . . . . . . . . 10

2.2 Radial width of the basilar membrane. . . . . . . . . . . . . . . . 11

2.3 Cross section of a mammalian organ of corti. . . . . . . . . . . . . . 19

2.4 Array of damped oscillators. . . . . . . . . . . . . . . . . . . . 20

2.5 Effective mass of the basilar membrane. . . . . . . . . . . . 21

2.6 Damping properties of the basilar membrane. . . . . . . . . . 22

2.7 Virtual stiffness of the basilar membrane. . . . . . . . . . . . . 23

2.9 Outer hair cell driven force caused by hair bundle deflection. . . . . . . . 26

2.10 The Mass of the tectorial membrane. . . . . . . . . . . . . . . 27

2.11 The viscosity of the secondary oscillator. . . . . . . . . . . . . 28

2.12 The effective stiffness of the subordinate system. . . . . . . . . . . 29

2.13 Sine-cosine decomposition. . . . . . . . . . . . . . . . . . . 30

3.1 Refinement of the upper part of the mesh. . . . . . . . . . . . 38

3.3 Arrangement of the local nodes on line-segments as well as triangular elements for both, linear and quadratic polynomial spaces. . . . . . . . . . . 49

4.1 Pressure and forces acting on the basilar membrane. . . . . . . . . 82

4.2 Forces acting on the basilar membrane in the active cochlea model. . . . . . 83

4.3 Envelopes and phases of the traveling wave at different frequencies in the passive cochlea. . . . . . . . . . . . . . . . 84

4.4 Maximal displacement of the basilar membrane at different stimulation amplitudes.

4.5 Frequency position map. . . . . . . . . . . . . . . . . 86

4.6 Envelopes of the traveling wave at different activity parameters. . . . . . . 88

4.7 Envelopes and phases of the traveling wave at different frequencies in the active cochlea. . . . . . . . . . . . . . . . . . . . 89

4.8 Gain of the basilar membrane amplitude. . . . . . . . . . . . . 90

4.9 Acoustic flow motion within the Stokes boundary layer. . . . . . . . . . . . 91 
4.10 Difference between the Lagrangian and the Eulerian velocity. . . . . . . . . 94

4.11 Mass source driven mean flow. . . . . . . . . . . . . . . . . . . 95

4.12 Reynolds stresses within the Stokes boundary layer. . . . . . . . . . . . . 97

4.13 Lighthill's estimate of the maximal mean velocity at different outer hair cell

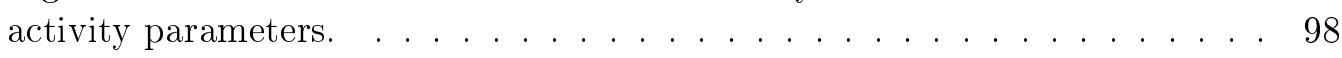

4.14 Second order mean motion . . . . . . . . . . . . . . . . . . . . 100

4.15 Maximal mean velocity at different outer hair cell activities. . . . . . . . . . 101

4.16 Maximal mean velocity at different stimulation amplitudes. . . . . . . . . 102

A.1 Current state after $\frac{1}{4} T$ seconds at $1024 \mathrm{~Hz}$ stimulation frequency. . . . . . . 107

A.2 Current state after $\frac{1}{2} T$ seconds at $1024 \mathrm{~Hz}$ stimulation frequency. . . . . . . 108

A.3 Current state after $\frac{3}{4} T$ seconds at $1024 \mathrm{~Hz}$ stimulation frequency. . . . . . . 108

A.4 Current state after $T$ seconds at $1024 \mathrm{~Hz}$ stimulation frequency. . . . . . . . 109

A.5 Current state after $\frac{5}{4} T$ seconds at $1024 \mathrm{~Hz}$ stimulation frequency. . . . . . . 109

A.6 Current state after $\frac{3}{2} T$ seconds at $1024 \mathrm{~Hz}$ stimulation frequency. . . . . . . 110

A.7 Current state after $\frac{7}{4} T$ seconds at $1024 \mathrm{~Hz}$ stimulation frequency. . . . . . 110

A.8 Current state after $2 T$ seconds at $1024 \mathrm{~Hz}$ stimulation frequency. . . . . . . . 111

A.9 Acoustic field at $128 \mathrm{~Hz}$ stimulation frequency in the passive model. . . . . 112

A.10 Acoustic field at $256 \mathrm{~Hz}$ stimulation frequency in the passive model. . . . . . 113

A.11 Acoustic field at $512 \mathrm{~Hz}$ stimulation frequency in the passive model. . . . . . 114

A.12 Acoustic field at $1024 \mathrm{~Hz}$ stimulation frequency in the passive model. . . . . 115

A.13 Acoustic field at $2048 \mathrm{~Hz}$ stimulation frequency in the passive model. . . . . 116

A.14 Acoustic field at $4096 \mathrm{~Hz}$ stimulation frequency in the passive model. . . . 117

A.15 Acoustic field at $8192 \mathrm{~Hz}$ stimulation frequency in the passive model. . . . . 118

A.16 Displacement of the basilar membrane at $128 \mathrm{~Hz}$ stimulation frequency in the passive model. . . . . . . . . . . . . . . . . . . . 119

A.17 Displacement of the basilar membrane at $256 \mathrm{~Hz}$ stimulation frequency in the passive model. . . . . . . . . . . . . . . . . . . 120

A.18 Displacement of the basilar membrane at $512 \mathrm{~Hz}$ stimulation frequency in the passive model. . . . . . . . . . . . . . . . . . . . . 120

A.19 Displacement of the basilar membrane at $1024 \mathrm{~Hz}$ stimulation frequency in the passive model. . . . . . . . . . . . . . . . . . . . . . 121

A.20 Displacement of the basilar membrane at $2048 \mathrm{~Hz}$ stimulation frequency in the passive model. . . . . . . . . . . . . . . . . . . . 121

A.21 Displacement of the basilar membrane at $4096 \mathrm{~Hz}$ stimulation frequency in the passive model. . . . . . . . . . . . . . . . . 122

A.22 Displacement of the basilar membrane at $8192 \mathrm{~Hz}$ stimulation frequency in the passive model. . . . . . . . . . . . . . . . . . . . 122

A.23 Acoustically driven flows at $128 \mathrm{~Hz}$ in the passive model. . . . . . . . . . . 123

A.24 Acoustically driven flows at $256 \mathrm{~Hz}$ in the passive model. . . . . . . . . 123

A.25 Acoustically driven flows at $512 \mathrm{~Hz}$ in the passive model. . . . . . . . . . . 124

A.26 Acoustically driven flows at $1024 \mathrm{~Hz}$ in the passive model. . . . . . . . . . . 124

A.27 Acoustically driven flows at $2048 \mathrm{~Hz}$ in the passive model. . . . . . . . . 125

A.28 Acoustically driven flows at $4096 \mathrm{~Hz}$ in the passive model. . . . . . . . . . 125 
A.29 Acoustically driven flows at $8192 \mathrm{~Hz}$ in the passive model. . . . . . . . . 126

B.1 Acoustic field at $128 \mathrm{~Hz}$ stimulation frequency in the passive model. . . . . . 128

B.2 Acoustic field at $256 \mathrm{~Hz}$ stimulation frequency in the passive model. . . . . . 129

B.3 Acoustic field at $512 \mathrm{~Hz}$ stimulation frequency in the passive model. . . . . . 130

B.4 Acoustic field at $1024 \mathrm{~Hz}$ stimulation frequency in the passive model. . . . . 131

B.5 Acoustic field at $2048 \mathrm{~Hz}$ stimulation frequency in the passive model. . . . . 132

B.6 Acoustic field at $4096 \mathrm{~Hz}$ stimulation frequency in the passive model. . . . . 133

B.7 Acoustic field at $8192 \mathrm{~Hz}$ stimulation frequency in the passive model. . . . . 134

B.8 Displacement of the basilar membrane at $128 \mathrm{~Hz}$ stimulation frequency in the active model. . . . . . . . . . . . . . . . . 135

B.9 Displacement of the basilar membrane at $256 \mathrm{~Hz}$ stimulation frequency in the active model . . . . . . . . . . . . . . . . . 136

B.10 Displacement of the basilar membrane at $512 \mathrm{~Hz}$ stimulation frequency in the active model. . . . . . . . . . . . . . . . . . . 136

B.11 Displacement of the basilar membrane at $1024 \mathrm{~Hz}$ stimulation frequency in the active model. . . . . . . . . . . . . . . . . 137

B.12 Displacement of the basilar membrane at $2048 \mathrm{~Hz}$ stimulation frequency in the active model. . . . . . . . . . . . . . . . . 137

B.13 Displacement of the basilar membrane at $4096 \mathrm{~Hz}$ stimulation frequency in the active model. . . . . . . . . . . . . . . . . . . . 138

B.14 Displacement of the basilar membrane at $8192 \mathrm{~Hz}$ stimulation frequency in the active model. . . . . . . . . . . . . . . . . . . 138

B.15 Acoustically driven flows at $128 \mathrm{~Hz}$ in the active model. . . . . . . . . . . . 139

B.16 Acoustically driven flows at $256 \mathrm{~Hz}$ in the active model. . . . . . . . . . . . 139

B.17 Acoustically driven flows at $512 \mathrm{~Hz}$ in the active model. . . . . . . . . . . . . 140

B.18 Acoustically driven flows at $1024 \mathrm{~Hz}$ in the active model. . . . . . . . . . . . 140

B.19 Acoustically driven flows at $2048 \mathrm{~Hz}$ in the active model. . . . . . . . . . . . 141

B.20 Acoustically driven flows at $4096 \mathrm{~Hz}$ in the active model. . . . . . . . . . . 141

B.21 Acoustically driven flows at $8192 \mathrm{~Hz}$ in the active model. . . . . . . . . . 142 


\section{List of Tables}

2.1 Constants that are needed for the simulation of the fluid dynamics. . . . . . 13

3.1 Linear basis functions on line-segments. . . . . . . . . . . . . . . 50

3.2 Quadratic basis functions on line-segments. . . . . . . . . . . . 51

3.3 Linear basis functions on triangular elements. . . . . . . . . . . . . 51

3.4 Quadratic basis functions on triangular elements. . . . . . . . . . . 52 


\section{List of Algorithms}

3.1 Global algorithm for the calculation of acoustic streaming within the inner

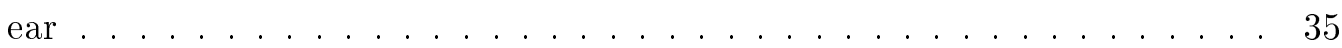

3.2 Recursive algorithm of the refinement . . . . . . . . . . . . . . . 40

3.3 Generalized Minimal Residual Method . . . . . . . . . . . . . . . . . 72

3.4 Incomplete LU decomposition . . . . . . . . . . . . . . . . . 76 


\section{Bibliography}

[1] J. Ashmore. Cochlear outer hair cell motility. Physiological reviews, 88(1):173, 2008.

[2] Satish Balay, Jed Brown, Kris Buschelman, William D. Gropp, Dinesh Kaushik, Matthew G. Knepley, Lois Curfman McInnes, Barry F. Smith, and Hong Zhang. PETSc Web page. http://www.mcs.anl.gov/petsc, August 2011.

[3] Klaus-Jürgen Bathe and P. Zimmermann. Finite-Elemente-Methoden. Springer, 2001.

[4] H.G. Boenninghaus and T. Lenarz. Hals-Nasen-Ohren-Heilkunde. Springer-Lehrbuch. Springer Medizin Verlag Heidelberg, 2007.

[5] F. Böhnke and W. Arnold. 3d-finite element model of the human cochlea including fluid-structure couplings. ORL J Otorhinolaryngol Relat Spec, 61(5):305-310, 1999.

[6] S. Boluriaan and P.J. Morris. Acoustic streaming: from rayleigh to today. International Journal of Aeroacoustics, 2(3):255-292, 2003.

[7] S. Boluriaan and P.J. Morris. Numerical simulation of acoustic streaming in high amplitude standing waves. AIAA Paper No. 2003-3152, 2003.

[8] Hartmut Bossel. Modellbildung und Simulation.: Konzepte, Verfahren und Modelle zum Verhalten dynamischer Systeme. Vieweg, 1994.

[9] CE Bradley. Acoustic streaming field structure: The influence of the radiator. The Journal of the Acoustical Society of America, 100:1399, 1996.

[10] F. Böhnke and C. Gerstenberger. Acoustic streaming in a viscous fluid-structure system. In M.M. Boone and TU Delft, editors, NAG/DAGA 2009 International Conference on Acoustics, pages 623-624. German Acoustical Society (DEGA), 2009.

[11] L. Cheng, R.D. White, and K. Grosh. Three-dimensional viscous finite element formulation for acoustic fluid-structure interaction. Computer methods in applied mechanics and engineering, 197(49-50):4160-4172, 2008.

[12] Nigel Paul Cooper and D. T. Kemp. Concepts and challenges in the biophysics of hearing: proceedings of the 10th International Workshop on the Mechanics of Hearing, Keele University, Staffordshire, UK, 27-31 July 2008. World Scientific, 2009. 
[13] P. Dallos. The active cochlea. The journal of neuroscience, 12(12):4575, 1992.

[14] Milton Van Dyke. Perturbation methods in fluid mechanics. Parabolic Press, 1975.

[15] Christiane Förster. Robust methods for fluid-structure interaction with stabilised finite elements. Stuttgart, 2007.

[16] C. Gerstenberger and F.-E. Wolter. Numerical simulation of acoustic streaming within a biological fluid-structure coupled system like the cochlea. In J. Becker-Schweitzer and G. Notbohm, editors, Fortschritte der Akustik - DAGA 2011, number 2, pages 817-818. Deutsche Gesellschaft für Akustik e.V., 2011.

[17] E. Givelberg and J. Bunn. A comprehensive three-dimensional model of the cochlea* 1. Journal of Computational Physics, 191(2):377-391, 2003.

[18] D.D. Greenwood. A cochlear frequency-position function for several species-29 years later. The Journal of the Acoustical Society of America, 87:2592, 1990.

[19] Ralf Greve. Kontinuumsmechanik. Springer, 2003.

[20] W.L. Hallauer. Nonlinear mechanical behavior of the cochlea. Ph.d. thesis, Stanford University, 1974.

[21] J.C. Houbolt. A recurrence matrix solution for the dynamic response of elastic aircraft. Journal of the Aeronautical Sciences, 17:540-550, 1950.

[22] BM Johnstone, R. Patuzzi, and GK Yates. Basilar membrane measurements and the travelling wave. Hearing Research, 22(1-3):147-153, 1986.

[23] P. Knabner and L. Angermann. Numerik partieller Differentialgleichungen: Eine anwendungsorientierte Einführung. Springer-Lehrbuch. Springer, 2000.

[24] D. Köster. Numerical simulation of acoustic streaming on surface acoustic wave-driven biochips. SIAM Journal on Scientific Computing, 29:2352, 2007.

[25] J. Lighthill. Acoustic streaming in the ear itself. Journal of Fluid Mechanics, 239(1):551-606, 1992.

[26] S.J. Lighthill. Acoustic streaming. Journal of Sound and Vibration, 61(3):391-418, 1978.

[27] K.M. Lim and C.R. Steele. A three-dimensional nonlinear active cochlear model analyzed by the wkb-numeric method. Hearing research, 170(1):190-205, 2002.

[28] F. Mammano and R. Nobili. Biophysics of the cochlea: linear approximation. The Journal of the Acoustical Society of America, 93:3320, 1993.

[29] D. Manoussaki, R.S. Chadwick, D.R. Ketten, J. Arruda, E.K. Dimitriadis, and J.T. O'Malley. The influence of cochlear shape on low-frequency hearing. Proceedings of the National Academy of Sciences, 105(16):6162, 2008. 
[30] Philip M. Morse and K. Uno Ingard. Theoretical acoustics. McGraw Hill, 1968.

[31] R. Nobili. Cochlear dynamics simulated in the time domain (rough model). http://147.162.36.50/cochlea/cochleapages/download/td_coch.zip, August 2011.

[32] R. Nobili and F. Mammano. Biophysics of the cochlea II: Stationary nonlinear phenomenology. Journal of the Acoustical Society of America, 99(4):2244-2255, 1996.

[33] R. Nobili, A. Vetešnik, L. Turicchia, and F. Mammano. Otoacoustic emissions from residual oscillations of the cochlear basilar membrane in a human ear model. JAROJournal of the Association for Research in Otolaryngology, 4(4):478-494, 2003.

[34] A.L. Nuttall and D.F. Dolan. Steady-state sinusoidal velocity responses of the basilar membrane in guinea pig. The Journal of the Acoustical Society of America, 99:1556, 1996.

[35] Lord Rayleigh. Theory of Sound, volume 2. Macmillan and co., 1896.

[36] J. N. Reddy and D. K. Gartling. The Finite Element Method in Heat Transfer and Fluid Dynamics, Third Edition. CRC Press, 2010.

[37] Junuthula Narasimha Reddy. An introduction to continuum mechanics: with applications. Cambridge University Press, 2008.

[38] L. Robles and M.A. Ruggero. Mechanics of the mammalian cochlea. Physiological reviews, 81(3):1305, 2001.

[39] Y. Saad. Iterative methods for sparse linear systems. PWS Pub. Co., 1996.

[40] Y. Saad and M.H. Schultz. Gmres: A generalized minimal residual algorithm for solving nonsymmetric linear systems. SIAM journal on scientific computing, 7(3):856$869,1986$.

[41] Jean Salençon. Handbook of continuum mechanics: general concepts, thermoelasticity. Springer, 2001.

[42] A. Schmidt and K.G. Siebert. Design of adaptive finite element software: The finite element toolbox ALBERTA, volume 42. Springer Verlag, 2005.

[43] A. Schmidt, K.G. Siebert, D. Köster, and C.-J. Heine. Alberta 3.0 - release candidate 6. http://www.numa.uni-due.de/downloads/alberta/alberta-3.0-rc6.tar.gz, June 2009 .

[44] A. Schmidt, K.G. Siebert, D. Köster, and C.-J. Heine. Alberta 3.0: Technical manual. http://www .numa.uni-due.de/downloads/alberta/alberta-man.pdf, June 2009.

[45] PM Sellick, R. Patuzzi, and BM Johnstone. Measurement of basilar membrane motion in the guinea pig using the Mossbauer technique. The journal of the acoustical society of America, 72:131, 1982. 
[46] PM Sellick, GK Yates, and R. Patuzzi. The influence of Mossbauer source size and position on phase and amplitude measurements of the guinea pig basilar membrane. Hearing Research, 10(1):101-108, 1983.

[47] Joseph H. Spurk and Nuri Aksel. Fluid mechanics. Springer, 2007.

[48] J. Tonndorf. Nonlinearities in cochlear hydrodynamics. The Journal of the Acoustical Society of America, 47:579, 1970.

[49] M. Ulfendahl. Mechanical responses of the mammalian cochlea. Progress in neurobiology, 53(3):331-380, 1997.

[50] G. Von Békésy. Experiments in hearing. 1960.

[51] Kai Willner. Kontinuums- Und Kontaktmechanik: Synthetische Und Analytische Darstellung. Springer, 2003.

[52] J. Wysocki. Dimensions of the human vestibular and tympanic scalae. Hearing research, 135(1-2):39-46, 1999.

[53] T. Yano. Turbulent acoustic streaming excited by resonant gas oscillation with periodic shock waves in a closed tube. The Journal of the Acoustical Society of America, 106(1):L7-L12, 1999.

[54] Y.J. Yoon, C.R. Steele, and S. Puria. Feed-forward and feed-backward amplification model from cochlear cytoarchitecture: an interspecies comparison. Biophysical journal, 100(1):1-10, 2011.

[55] O.C. Zienkiewicz, R.L. Taylor, R.L. Taylor, and P. Nithiarasu. The finite element method for fluid dynamics. The Finite Element Method. Elsevier ButterworthHeinemann, 2005.

[56] O.C. Zienkiewicz, R.L. Taylor, R.L. Taylor, and J.Z. Zhu. The finite element method: its basis and fundamentals. The Finite Element Method. Elsevier ButterworthHeinemann, 2005. 


\section{Index}

A

acoustic intensity

96

acoustic streaming

acoustic streaming subproblem .....17, 31 acoustic subproblem ...............17

active model .....................80

B

basilar membrane... see cochlear partition basis function....................

Lagrange family ..............50

boundary ......................... 10

boundary condition ..............15

C

characteristic frequencies............23

characteristic place ................86

cochlear amplifier .................. 19

cochlear partition .................10

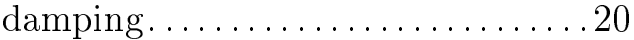

external forces .................23

inertia ......................20

stiffness ....................23

computational model ..............5, 7

conservation of angular momentum ....12

conservation of linear momentum ......112

conservation of mass ...............12

constitutive relation ................13

continuum ......................12

coupling .....................6.

strong ...................61

weak ...................61

D

density field .....................12 dimension......................

displacement

of the basilar membrane ......... 15

of the stapes.................15

dual pairing $\ldots \ldots \ldots \ldots \ldots \ldots \ldots \ldots, 43$

\section{E}

effective slip flow $\ldots . \ldots \ldots \ldots \ldots \ldots$. 2

Einstein summation convention ........43 element.................. see simplex equilibrium state.................29

Eulerian specification of motion ....... 112

F

finite difference scheme $\ldots \ldots \ldots \ldots \ldots 668$

finite element method $\ldots \ldots \ldots \ldots \ldots \ldots .33$

finite element space................33

force source distribution......... 31, 78

form function.......... see basis function frequency

angular.......................17

G

geometry $\ldots \ldots \ldots \ldots \ldots \ldots \ldots \ldots, \ldots$

GMRES $\ldots \ldots \ldots \ldots \ldots \ldots \ldots \ldots \ldots+70 \mathrm{f}$.

restarted $\ldots \ldots \ldots \ldots \ldots \ldots \ldots \ldots, 74$

Gram-Schmidt process .............71

Green's first identity.................41

$\mathbf{H}$

hair bundle

deflection....................24

Houbolt method .................69 
I

ILU decomposition ............... 75 interpolation function .. see basis function K

Kronecker delta operator............56 Krylov subspace $70 \mathrm{f}$. $\mathbf{L}$

Lagrangian specification of motion .....12 LBB condition ................. 54 level of fill .................... .77 longitudinal ..................... .9.

\section{M}

mach number .16 mass source distribution......... 31, ,78 material frame indifference...........14 mesh .......................... 35

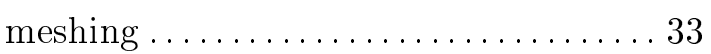
mixed formulation $\ldots \ldots \ldots \ldots \ldots \ldots \ldots$.61 $\mathbf{N}$

no-slip-condition .................15 node

global $\ldots \ldots \ldots \ldots \ldots \ldots \ldots \ldots \ldots . \ldots \ldots 2$

local ......................50

numerical quadrature ..............68

\section{O}

outer hair cell driven force ........24, 28 outer hair cell motility.............24

damping....................27

inertia ..................... 27

stiffness.................... $27 \mathrm{f}$.

$\mathbf{P}$

passive model....................80

Perturbation Expansion..............16

preconditioning . . . . . . . . . . . . .

pressure field . . . . . . . . . . . . . . . . . 13
$\mathbf{R}$

radial ........................... .9

refinement ....................... . 37

Reynolds number ..................16

Reynolds stress................1, 97

normal stress component ........9797

shear stress component ...........97

$\mathbf{S}$

sigmoid function ............. 26, 29,

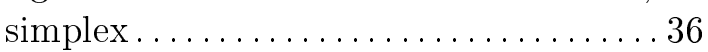

barycentric ................ 36

normalized ................ 36

Sine-Cosine Decomposition .......... 77

sine-cosine decomposition . ...........30

small signal sound speed .......... $15 \mathrm{f}$.

Sobolev-norm.....................46

Sobolev-space....................47

stereocilia...............see hair bundle

Stokes boundary layer........... 39, 90,

strain rate ......................13 stress

deviatoric ................... 13

hydrostatic ................. 13

stress tensor . . . . . . . . . . . . . . . 12

system of damped oscillators.........19

$\mathbf{T}$

temporal discretization $\ldots . . \ldots \ldots \ldots \ldots 68$

test-function .....................41

thermal equation of state ..........14

transversal ......................

traveling wave............... 39 , 86

triangle................... see simplex

triangulation

regular

V

variational formulation ...........34, 41 velocity field $\ldots . \ldots \ldots \ldots \ldots \ldots \ldots \ldots .12$ vertex

hanging ..................... 37 
viscosity $\ldots \ldots \ldots \ldots \ldots \ldots \ldots \ldots \ldots \ldots \ldots$

bulk ........................ 14

shear....................... 14

W

weak form..... see variational formulation 


\section{Curriculum Vitae}

\section{Personal Data}

Christian Gerstenberger

Date of Birth: January 30, 1983

Place of Birth: Hannover, Germany

\section{Education}

Since 2008 University of Hannover, Germany

Doctoral Study at the Division of Computer Graphics

2002-2008 University of Hannover, Germany

Studies of Mathematics and Computer Science

Specialization: Image Processing

April 2008, Graduation, Degree "Dipl.-Math."

2000-2002 Carl-Friedrich-Gauß School, Hemmingen, Germany

Core Subjects: Mathematics, Physics

June 2002, Graduation "Abitur" 